\title{
31st Meeting of the Canadian Congress of Neurological Sciences
}

\section{ORAL PRESENTATIONS}

Thursday, June 27
A. Peripheral Nerve and
Neurophysiology (14:00 -17:00)
A-01 to $A-11$
B. Cerebrovascular Disease and
Clinical Neurogenetics (14:00 - 17:00)
B-01 to B-11
C. Pediatric Neurology $(14: 00-17: 00)$
C-01 to $\mathrm{C}-1$
D. Surgical Oncology $(14: 00-17: 00)$
D-01 to D-06
E. Cerebrovascular Surgery (14:00 - 17:00)
E-01 to E-11

Friday, June 28, 14:00 - 17:00

F. Clinical Neurology and Experimental

Therapeutics (14:00 - 17:00) F-01 to F-11

G. Epilepsy (14:00 - 17:00) G-01 to G-11

H. General Neurosurgery $(13: 30-16: 30)$. $\mathrm{H}-01$ to $\mathrm{H}-11$

I. Pediatric Neurosurgery $(14: 00-17: 00)$ .I -01 to $\mathrm{I}-1 \mathrm{I}$

\section{Oral Presentations}

\section{A. PERIPHERAL NERVE AND NEUROPHYSIOLOGY}

\section{A.-01}

\section{Local Events in Experimental Neuromas}

\section{D.W. Zochodne, C. Nguyen, C. Cheng (Calgary, Alberta)}

Background: The local environment of an injured peripheral nerve trunk may determine the success of regenerative sprouting. In this report we correlate previous findings of CGRP hyperemia, mast cell accumulation and angiogenesis with neuroma development.

Methods: Experimental neuromas were created in the sciatic nerve of adult male Sprague-Dawley rats by sciatic nerve section with resection of the distal nerve and its branches to prevent regeneration. Methods for analysis of local perfusion, CGRP, mast cells and microvessels have been described. Further studies were made of SP content, microvessel size distribution, CGRP origin and axonal sprouting by light microscopy.

Results: (1) CGRP and SP accumulation accompanied early (48h) hyperemia in "neuromas" before actual nerve trunk enlargement or cellular proliferation. CGRP accumulation did not occur with additional prior proximal nerve section. (2) Later nerve trunk enlargement and neuroma development accompanied angiogene-

\section{Session I}

\section{POSTER PRESENTATIONS}

Thursday, June 27,

(07:00 - 17:00, Authors standing by 13:00 - 14:00)

- Basic Neurophysiology . . . . . . . . . . P-01 to P-013

- General Neurosurgery ... . . . . . . . . . . P-013 to P-029

- Multiple Sclerosis and Neurovirology ... . . P-030 to P-038

- Pediatric Neurology ... . . . . . . . . . . . P-039 to P- 046

- Pediatric Neurosurgery ... . . . . . . . . . . P-047 to P-056

- Peripheral Nerve and Muscle . . . . . . . . . P-057 to P-075

- Surgical Oncology ... . . . . . . . . . . . P-076 to P-097

Session II

Friday, June 28

(07:00 - 17:00, Authors Standing By 13:00 - 14:00)

- Cerebrovascular Disease ... . . . . . . . ...P-098 to P-110

- Epilepsy ..................... P-111 to P-137

- Experimental Therapeutics .............. P-138 to P-143

- General Neurology ... . . . . . . . . . . . . . . P-144 to P-161

- Neurological Nursing - General . . . . . . . . P-162 to P-175

- Surgical Cerebrovascular ..............P-176 to 199

Note: The Canadian Congress of Neurological Sciences has invited the The Canadian Association of Neuroscience Nurses to join in the Poster Session on Friday, June 28. The posters have been integrated into the CCNS posters and the titles are listed in this book followed by (CANN) to indicate the affiliation.

sis, mast cell proliferation, and the formation of axonal regenerative clusters. (3) Angiogenesis was confirmed with quantitative analysis of India ink perfused microvessels. Neuromas had rises in the total number of perfused microvessels, the number of microvessels of 2-4 and 4-6 micron diameter, and the percentage of microvessels of 2-4 micron diameter at $7 \mathrm{~d}$. New microvessels were often observed with mast cells and axonal regenerative clusters at $14 \mathrm{~d}$ splaying apart the layers of the previous perineum.

Conclusions: CGRP accumulation from antegrade axoplasmic transport precedes proliferative events in experimental neuromas. There is a shared timetable of events with nerve enlargement, and proliferation of mast cells, new microvessels and axonal sprouts perhaps from overlapping shared trophic signals.

Supported by MDAC, AHFMR

\section{A-02}

The Role of Anti- $P_{0} A B$ in the Pathogenesis of EAN.

A.F. Hahn, D. Lovgren, L. Wilkie, (London, Ontario) J. ArChelos, H.P. Hartung (Wuerzburg, Germany)

Background /Objective: Recent evidence suggests that $\mathrm{P}_{0}$ a major peripheral myelin protein, may act as autoantigen and provoke cell-mediated and humoral immune responses in EAN9 (Linnington 1992; Archelos 1993). In this study we examined the potential synergism of activated $\mathrm{CD}_{4}+\mathrm{SP}_{26}$ sensitized 
$\mathrm{T}$ lymphocytes and circulating anti- $\mathrm{P}_{0} \mathrm{AB}$ in adoptive transfer EAN (Hahn et al, 1993)

Method/Design: AT-EAN was induced in Lewis rats by intracardiac injection of $2{\mathrm{X} 10_{5}}_{5} \mathrm{SP}_{26}$ sensitized $\mathrm{T}$ lymphoctes. On day 4 post cell transfer 11 rats were injected systemically with anti- $\mathrm{P}_{0} \mathrm{AB}(3.5 \mathrm{mg}$ each) whereas control rats received cells alone. Clinical scores and weights were recorded daily and the pathology was assessed on day 11 post cell transfer.

Results: Experimental and control animals developed typical clinical EAN of mild to moderate severity. Clinical scores were significantly greater $(\mathrm{p}<0.05)$ in rats injected with anti- $\mathrm{P}_{0} \mathrm{AB}$ and enhanced demyelination and axonal degeneration was observed in lumbosacral nerve roots and sciatic nerves.

Conclusion: Circulating $\mathrm{AB}$ directed towards the extracellular domaine of $\mathrm{P}_{0}$ myelin protein may be of pathogenetic significance in EAN.

\section{A-03}

\section{The Effect of Eccentric Contractions on Spastic Muscle}

\section{A. VuJNOVICH, L. ROCHESTER (Auckland, New Zealand)}

Background: The purpose of this study was to investigate changes in the recruitment and excitability of motoneurons after eccentric contractions in normal subjects and spastic subjects.

Methods: Subjects were seated with the hip in $120^{\circ}$ flexion and the foot at $20^{\circ}$ plantarflexion. Hoffmann reflexes (H-reflex) were elicited using by a constant stimulus using standard criteria'. Eccentric contractions of the plantarflexors were performed on the KinCom at a velocity of $30^{0} \mathrm{~s}^{-1}$ and H-reflexes were taken before, and after. Mean peak-to-peak H-reflex amplitudes were compared using ANOVA $(\mathrm{P}<0.01)$.

Results: The amplitude of the $\mathrm{H}$ reflex was significantly increased following eccentric contractions in those with spasticity while normal subjects showed no change. Preliminary investigations indicate a significant increase in the gain of the monosynaptic reflex in those with spasticity.

Conclusions: Presynaptic inhibition has been shown to decrease with a lesion of the spinal cord although is unaffected in hemiplegics ${ }^{2}$. The increase in excitability of the motor pool following eccentric contractions may facilitate the excitatory effect of descending corticospinal pathways in activating the same motor pool during the performance of voluntary movement.

I. Hugon, M., In New Developments in Electromyography and Clinical Neurophysiology (ed. Desmedt, J.E.) Vol 3, 277-293, Karger, Basel, 1973)

2. Faist, M., Mazevet, D., Dietz, V., Pierrot-Deseilligny, E., Presynaptic Inhibition in Spasticity. Brain 2: 220-229, 1994

\section{A-04}

Initial and Sinusoidal Vestibulo-ocular Reflex Following Focal Brainstem Lesions

N. Torun, J.A. Sharpe (Toronto. Ontario)

Background: Most head movements made under natural viewing conditions are of high frequency. In order to preserve clear vision during head motion, the vestibulo-ocular reflex (VOR) must produce compensatory smooth eye movements with a short latency and the ratio of eye velocity to head velocity (VOR gain) must approximate 1.

Methods: We investigated the initial $80 \mathrm{msec}$ of VOR, and lower frequency steady state sinusoidal VOR (at $0.5,1.0$ and 2.0 Hertz) in 12 patients with focal brainstem lesions, shown by MRI. Their ocular motor deficits were grouped into four categories: conjugate gaze palsy, sixth nerve palsy, lateral medullary syndrome and gaze-evoked nystagmus. Horizontal eye movements were recorded using a magnetic search coil technique. The VOR was induced by transient impulsive stimulation to obtain initial VOR; and by active head shaking for steady-state sine waves. Patients attained a mean peak head acceleration of $290 \mathrm{deg} / \mathrm{sec} / \mathrm{sec}$ during transient impulsive stimulation. Both initial VOR and sinusoidal VOR were recorded with visual enhancement, and in darkness. We used a control group of 12 normal volunteer subjects (age range 29-73 years, mean 45, SD 16). Using prediction intervals, we compared the results for each patient to those obtained from the control group; $p<0.05$ was considered to be significant.

Results: Initial VOR detected asymmetry that was not detectable on clinical examination or by sinusoidal VOR. Two patients with unilateral saccadic palsy had significantly low VOR gains in both directions in all testing conditions.

Conclusions: The initial VOR is a sensitive test of brainstem function. It may be impaired in cases where oculocephalic manoeuvres produce full-range eye movements, as these test the visually enhanced VOR. It can also be impaired in patients with brainstem lesions when ocular motor examination does not reveal supranuclear deficits.

\section{A-05}

Functional MRI Investigation of the Generators of the Transient and Steady State Visual Evoked Potentials

W.J. LogAN (Toronto, Ontario), K.K. Kwong, B.R. Rosen, V.S. CAVINEss, JR. (Boston, Massachusetts)

Most clinical studies of visual evoked potentials (VEPs) evaluate the transient VEP to flash or pattern stimuli. The generators for these have been found in functional imaging experiments to involve different areas of visual cortex. Steady state flash VEPs can also be determined and these appear to have somewhat different clinical correlates and neurophysiological characteristics. Most functional imaging studies of vision have employed steady state visual stimuli. The present investigation was undertaken to compare the brain activation produced by steady state flash with that by transient flash and pattern reversal stimuli.

The visual activation produced by stroboscopic or other flash stimuli at $10-20 \mathrm{~Hz}$ was compared to flash or pattern stimuli at $1-2 \mathrm{~Hz}$ in eight adult volunteers. The activation was determined using a MRI technique which detects blood oxygenation level changes. A 1.5 T GE MR imager equipped with echoplanar fast imaging and a head coil were used to obtain multiple slices of occipital lobe. Activations produced by different stimuli were compared in the same subject. 
Steady state stimuli often produced stronger activation than transient flash stimuli. The area of activation was larger and involved extrastriate regions. It appeared to have a topography different from transient flash but similar to transient pattern reversal activation.

These results suggest that steady state flash VEPs have a different generator than transient flash VEPs.

\section{A-06}

Motor Evoked Potentials Recorded Directly from the Spinal Cord Following Transcranial Magnetic Stimulation in Awake Human Subjects: Direct Evidence that Human Motor Cortical Excitability does not Change During Voluntary Contraction

D.A. Houlden, M.L. Schwartz, C.H. Tator, P. Ashby, W. MACKAY, M. FAZL (Toronto, Ontario)

Background: Muscle responses following transcranial magnetic stimulation (TCMS) are facilitated during voluntary muscle contraction. Facilitation is thought to be due to increased motor cortical excitability and/or increased excitability at the spinal motoneuron pool but the precise mechanism is not known.

Methods: Spinal cord evoked potentials (SCEPs) were recorded from an epidural dorsal column stimulating electrode at T8 following transcranial magnetic (TCMS) in 4 awake, neurologically intact subjects. Muscle recordings were concomitantly recorded from left biceps, triceps, first dorsal interosseous, tibialis anterior (TA) and soleus. The magnetic stimulator coil was positioned for optimal activation of left TA.

Results: At SCEP threshold, TCMS produced a D wave (direct activation of pyramidal cells) in one subject, a $D$ and first I wave $\left(\mathrm{I}_{1}\right.$, indirect activation of pyramidal cells) in another subject and $\mathrm{a} I_{1}$ wave in the other 2 subjects. Higher TCMS intensities produced a SCEP with 5 peaks $\left(D, I_{1}, I_{2}, I_{3}, I_{4}\right)$ in 3 subjects and 4 peaks in one subject (absent $\mathrm{I}_{4}$ ) and evoked muscle responses from the upper and lower extremity in all subjects. One subject performed ankle dorsiflexion (10\% maximum output of TA) during TCMS which resulted in facilitation of all muscle responses without changing the $D$ and I waves of the SCEP.

Conclusion: TCMS directly activates pyramidal cells. Motor cortical excitability does not change during facilitation of muscle responses.

\section{A-07}

Focal Fiber Loss and Axonal Regeneration is Prominent In Multifocal Motor Neuropathy with Conduction Block

I. Grant, G. Gruener, G. Suarez, W. Litchy, P. Dyck (Rochester, Minnesota)

Background: Multifocal motor neuropathy with conduction block (MMN-CB) is characterized by asymmetric limb weakness and atrophy without sensory loss, and electrophysiologic conduction block restricted to motor nerve fibers. An autoimmune mechanism is postulated. Pathologic data are limited, but thinly-myelinated fibers, onion bulbs, and inflammation have been reported. Demyelination is presumed to be the pathologic substrate of conduction block (CB)

Methods: Five male patients with MMN-CB, mean age 38 years, were studied. Mean duration of illness was 9.8 years. Surface nerve conduction studies were followed by selective fascicular nerve biopsy (median in three patients, ulnar in two) with intraoperative localization of $\mathrm{CB}$. Teased fiber, morphologic (paraffin and plastic sections) and immunohistochemical studies were performed.

Results: Striking focal myelinated fiber loss and regeneration were found in two cases, with slight endoneurial perivascular inflammation in one. Diffuse low-grade axonal de- and regeneration were found in the third case. Two cases showed no abnormalities. Segmental demyelination was not apparent.

Conclusion: Myelinated fiber loss is prominent and focal in some cases of MMN-CB, and explains denervation in cases of long duration. The absence of demyelination is unexpected; possible causes include tissue sampling or a physiologic mechanism of $\mathrm{CB}$, e.g. channel blockade.

\section{A-08}

\section{Activity-Dependent Changes in Impulse Conduction in a Focal Nerve Lesion.}

T. Miller (London, Ontario), M. Kiernan, I. Mogyros, D. BURKE (Sydney, Australia).

Background: Conduction of brief trains of impulses causes an activity dependent depression in the excitability of axons. The present study was undertaken to determine whether, in patients with a focal nerve lesion, the axonal hyper-polarization produced by conduction of brief trains would result in conduction block in cutaneous afferents, thus indicating a site of impaired safety margin for impulse transmission.

Methods: A conditioning train of 10 supramaximal stimuli at $200 \mathrm{~Hz}$ was evaluated in 25 patients with focal conduction slowing across the carpal tunnel segment and 25 normal subjects.

Results: There was a significant correlation between the increase in latency and the severity of the compression neuropathy. In addition, there were similar linear relationships between the activity-dependent amplitude reduction and the corresponding change in latency for both the patients and the control subjects, indicating that there was no need to invoke additional factors to those operating in the control subjects to explain the greater amplitude depression in patients.

Conclusion: The magnitude of amplitude depression can be explained by temporal dispersion and activity-dependent conduction block may play little role as to the cause for the pathologically large amplitude depression seen in the patient population. The slowing of conduction was more analogous to slowing produced by acute compression than demyelination. 


\section{A-09}

\section{Studies of Sural Nerve Blood Flow in Human Neuropathy}

D.W. Zochodne, M. Theriault, J. Dort, G. Sutherland (Calgary, Alberta)

Objective: To measure local in situ blood flow of human sural nerves in vasculitic neuropathy (VN) and other neuropathies (ON) prior to nerve resection for biopsy.

Background: Techniques for measuring nerve blood flow in animal models of disease have been employed for several years but there has been little correlation with human neuropathy. Laser Doppler flowmetry (LDF) provides a relative RBC flux signal that correlates with invasive quantitative flow measurements in animal models and does not harm patients or the nerve sample. A mean LDF value acquired by multiple (8-10) standardized measurements from sites on the epineurial surface of an intact exposed human sural nerve prior to biopsy provides quantitative RBC flux data that can be compared among patients.

Methods: LDF values were collected in a standardized fashion in conscious human subjects undergoing diagnostic sural nerve biopsy or a research biopsy for a drug trial in diabetic neuropathy. Three patients had VN and 30 had $\mathrm{ON}$ : unclassified neuropathies (5), tomaculous neuropathy (1), CIDP (2), mild diabetic neuropathy (22). VN was diagnosed on the basis of clinical electrophysiological and histological criteria.

Results: Among ON, LDF measured $155 \pm 4$ perfusion units (PU). There were no significant differences in LDF values among different neuropathies within this group (including diabetic subjects). One sural nerve was treated with epineurial epinephrine before LDF and biopsy: the LDF value was reduced $>2$ SD below the ON mean (85 PU). Mean LDF in VN measured $102 \pm 12$ PU indicating a significant reduction $(\mathrm{p}=0.0006)$ overall (one of the patients had only a mild reduction: $130 \mathrm{PU}$ ).

Conclusions: LDF measurements of human sural nerve blood flow can provide reliable quantitative RBC flux data provided signals are acquired in a highly standardized fashion. The technique is safe and does not impair histological quality. Patients with VN may have reduced sural nerve blood flow.

Supported by MDAC, AHFMR

\section{A-10}

Sex-Influenced Phenotype in a Large Nova Scotian Kindred with Autosomal Dominant Charcot-Marie-Tooth Disease (CMT1A)

P.E. Neumann, T.J. Benstead S.K. Gupta, D.L. Guernsey (Halifax, Nova Scotia)

Background: We evaluated 32 individuals with CharcotMarie-Tooth (CMT) disease, descendants of an affected Nova Scotia man born in 1831. Several instances occurred of boys being more severely affected than their mothers as seen in $\mathrm{X}$ linked CMT.

Methods: Molecular analysis used Southern blot analysis, and polymorphic DNA microsatellite markers on chromosomes $X$ and 17 . Nine women and 7 men were available for grading of reflexes and muscle strength. Analysis of variance for several parameters was performed grouping for age or gender.

Results: X-linked inheritance was excluded by two cases of male-to-male transmission and linkage analysis with polymorphic DNA markers on the X-chromosome. Southern blot and microsatellite analysis demonstrated DNA duplication of the pmp22 locus on chromosome 17 (CMT1A). Men were clinically more affected for several parameters including Achilles reflex $(p<0.05)$ and hand interossei strength $(p<0.04)$. Strength decreased more sharply in men when plotted against age.

Conclusions: This CMT1A kindred demonstrates a sex-influenced phenotype, with men more severely affected. Over-expression of the pmp22 gene may be sex-influenced in this instance. Molecular studies are underway to investigate mechanisms for this novel sex-influence of an autosomal dominant neuropathy.

\section{A-11}

A Novel Frameshift Mutation in Connexin-32 is Associated with X-Linked Charcot-Marie-Tooth Disease

S.K. Gupta, T.J. Benstead, A. Purdy P.E. Neumann, D.L. Guernsey (Halifax, Nova Scotia)

Background: Molecular analysis was performed on a 49 year old man with Charcot-Marie-Tooth and a pattern consistent with $X$-linked inheritance (CMTX). Point mutations in the gene encoding a gap junction protein connexin-32 (CX-32) are the putative cause of CMTX. We wished to determine whether this patient had a novel or previously reported point mutation.

Methods: The entire CX-32 coding region from the proband was amplified by polymerase chain reaction and sequenced.

Results: A single discrepancy from the published sequence, a deletion of a guanosine residue at codon 177 was detected. The observed deletion and consequent frameshift would result in an altered coding sequence from codon 178 with a predicted premature termination of the protein at codon 194. This point mutation has not been previously reported.

Conclusions: Our findings support the view that alterated CX32 function through a change in amino acid sequence or truncation of the protein may lead to a demyelinating peripheral neuropathy. Direct sequencing of the CX22 gene can confirm the clinical suspicion of CMTX. Several point mutations in the CX-22 gene are associated with CMTX. Molecular analysis of CMTX should be directed at a search for previously reported or novel mutations.

\section{B. CEREBROVASCULAR DISEASE AND CLINICAL NEUROGENETICS}

\section{B-01}

\section{Peak Systolic Velocity and Carotid Stenosis}

A.V. Alexandrov, D.S. Brodie, A. Mclean, J. Murphy, P. Hamilton (Toronto, Ontario)

Background: Recent results of the North American (NASCET) trial of carotid surgery questioned reliability of the 
peak systolic velocity (PSV) criteria for grading the severity of carotid stenosis and thus in predicting the risk of stroke.

Methods: We prospectively studied 40 consecutive patients who underwent both color coded duplex ultrasound (ATL HDI Ultramark-9) and intra-arterial carotid angiography. PSV was measured with angle correction at the site of the tightest narrowing. Angiograms were evaluated independently of carotid duplex results using the NASCET and Carotid Stenosis Index (CSI) methods.

Results: All three variables correlated with each other $(0.92$, $0.62,0.67, \mathrm{p}<0.0001$, Pearson correlation). PSV did not increase with mild $(<30 \%)$ carotid stenosis, then increased abruptly in the moderate and severe groups and decreased to $0 \mathrm{~cm} / \mathrm{sec}$ near occlusion. In a stepwise logistic regression model the linear, quadratic and cubic correlations were found at the significance level: $0.38,0.17,0.22$ for NASCET and $0.45,0.24,0.03$ for CSI, $p<0.01$. When normal and occluded vessels were excluded from the analysis, the exponential increase in stenotic velocities was found: 0.63 NASCET and 0.86 CSI $(p<0.0001$ between two coefficients).

Conclusions: PSV correlates well with the NASCET and CSI measurements allowing for closer correlation with the latter. The unsatisfactory correlation of PSV and NASCET measurements in a multicentre setting could therefore be explained by complex nature of these correlations and lack of standardized protocol to interpret the velocity data obtained with different types of equipment.

\section{B.02}

\section{Carotid and Vertebral Artery Dissections: A Comparative Study}

J. Maher (Winnipeg, Manitoba), B.W. Yoon, D.M. Pelz, J.H.W. Pexman, C.G. Drake, V. Hachinski (London, Ontario)

Background: We compared outcome and prognostic factors in patients with carotid and vertebral artery dissections.

Method: Medical records of patients with cervicocranial dissection were reviewed, 50 of whom had follow-up telephone interviews.

Results: Regarding patients with spontaneous dissections, 22 of 25 patients with carotid artery dissections (SCD) compared to 15 of 29 patients with vertebral artery dissections (SVD) had an excellent outcome $(p=0.007)$. One of 25 patients with SCD compared to 20 of 29 patients with SVD had haemorrhagic dissections $(\mathrm{p}=0.0001)$. Of patients with haemorrhagic SVD, 4 out of 5 patients who rebled did poorly, whereas 3 out of 15 who did not rebleed did poorly $(p=0.03)$. Regarding patients with traumatic dissections, 8 of 14 patients with carotid artery dissections compared to 4 of 6 patients with vertebral artery dissections had an excellent outcome $(p=0.6)$. Traumatic dissections were not haemorrhagic, and compared to spontaneous dissections rarely affected intracranial arteries $(\mathrm{p}=0.007)$.

Conclusions: Patients with spontaneous vertebral artery dissections have a significantly greater incidence of haemorrhagic dissection and a poorer outcome than those with spontaneous carotid artery dissections. Patients with traumatic carotid and vertebral artery dissections tend to have a similar outcome, possibly as such dissections are almost all extracranial and bland.

\section{B-03}

\section{Attenuation of Cerebrovascular Changes in Chronic Hypertension by Enalapril}

S. NAG (Toronto, Ontario)

Our recent studies have demonstrated that mural thickening of arteriolar walls in chronic hypertension is associated with increased deposition of extracellular matrix proteins such as fibronectin, laminin and collagen IV. An additional cerebral finding in chronic hypertension is the occurrence of multifocal areas with increased blood-brain barrier permeability to plasma proteins which may be associated with hypertensive encephalopathy. The present study was undertaken to determine which alterations are attenuated by antihypertensive therapy. Chronic renal hypertension was induced in rats and one group of hypertensive rats was treated with enalapril $(30 \mathrm{mg} / \mathrm{Kg})$, an angiotensin-converting enzyme inhibitor which was added to the drinking water. Treatment was initiated 2 weeks after the surgery to induce hypertension and continued for 5 weeks. Rats were then perfusion-fixed and their brains were removed and processed for morphological studies. The extracellular matrix proteins localised by immunohistochemistry were laminin, fibronectin and collagen IV. There was increased immunoreactivity for laminin, fibronectin and collagen IV in pial and intracerebral vessels of untreated hypertensive rats. Immunoreactivity was greatest in vessels in areas with breakdown of the bloodbrain barrier to plasma proteins. Most enalapril-treated hypertensive rats had lower blood pressures as compared to the untreated hypertensive rats. In addition, immunoreactivity for the extracellular matrix proteins was similar to the findings in normotensive controls. Thus treatment with enalapril attenuated the changes in extracellular matrix proteins that occurs in hypertension.

\section{B-04}

"The Use-Dependent Sodium Channel Blocker, Mexiletine Attenuates CA1 Damage Following Ischemia"

\section{H. Lesiuk, P. Stys, K. HewitT (Ottawa, Ontario)}

Sodium overload, and a reversal of the $\mathrm{Na}^{+} / \mathrm{Ca}_{2}{ }^{+}$exchanger may contribute to calcium influx and subsequent cell death following an ischemic insult. We assessed the efficacy of a usedependent $\mathrm{Na}$-channel blocker, mexiletine, in preventing $\mathrm{CA}_{1}$ hippocampal damage following stroke.

Methods: Male Sprague Dawley rats (300-450g) anaesthetized with sodium pentobarbital $(65 \mathrm{mg} / \mathrm{kg}$ ip) and artificially ventilated, underwent transient forebrain ischemia from bilateral carotid occlusion plus hypotension (blood presure $50 \mathrm{mmHg}$ ) for 10 minutes. Twenty minutes prior to ischemia, animals received either mexiletine $(80 \mathrm{mg} / \mathrm{kg}$, ip) $(n=12)$ or an equal volume of normal saline $(n=7)$. Cerebral temperature was maintained at 
$37.5+0.2$ degrees during ischemia, and until full postoperative recovery. Animals were allowed to recover for 7 days and then were sacrificed by perfusion fixation under anesthesia. Brains were removed and standard $7 \mu \mathrm{m}$ coronal $\mathrm{H} \& \mathrm{E}$ sections subsequently prepared. Neuronal damage was quantified in hippocampal area $C A_{1}$, expressed as the ratio of dead to total neurons.

Results: Control animals sustained $86 \pm 5 \%$ (mean \pm sem) $\mathrm{CA}_{1}$ neuronal injury. Pre-treatment with mexiletine reduced this significantly $(\mathrm{p}<0.02$, Mann-Whitney $\mathrm{U})$, by almost $50 \%$, to $45 \pm 9 \%$. We conclude that Na-channel blockade with mexiletine is an effective treatment for ischemic neuronal injury. This suggests that $\mathrm{Na}$ influx during ischemia is harmful, contributing to the pathophysiological events mediating cell death.

\section{B-05}

\section{Hyperhomocysteinemia - An Independent Risk Factor For Stroke}

R.H. Wilson, V. Markle, (Scarborough, Ontario), D.E.C. COLE, (Toronto, Ontario)

Background: The identification of new risk factors for stroke is an essential part of stroke research because the effectiveness of the treatment for acute stroke and the secondary prevention of stroke are both limited.

Method: A case report of a black man, without any risk factors for vascular disease, who sustained a basilar artery occlusion and despite intensive investigations no abnormal thrombic tendency was found.

Results: The blood homocysteine level was $52 \mathrm{~mol} / \mathrm{L}$ (reference range: $4-19 \mathrm{~mol} / \mathrm{L}$ ). The level returned to normal after folic acid supplement. Serial transcranial doppler studies indicated the basilar artery flow returned to normal.

Conclusion: It is concluded that hyperhomocysteinemia is an independent treatable risk factor for stroke. This will be discussed. It is suggested that stroke patients who have no vascular risk factors be tested for this condition. The genetic and nutritional basis for this condition indicates there is a high prevalence in the population. Thus general population screening may be helpful to prevent stroke by the prophylactic use of folic acid.

\section{B-06}

Susceptibility to Relapsing Progressive Multiple Sclerosis is Associated with Inheritance of a Gene Linked to the Variable Region of the T-Cell Receptor Beta Chain Locus

S.S . Beall, M.K. Hockertz, and D.W. Paty (Vancouver, British Columbia)

Background: The objective of the present research is to test the hypothesis that susceptibility to relapsing-progressive (RP) (but not relapsing-remitting (RR)) multiple sclerosis (MS) is associated with a gene linked to the $T c R \beta$ chain variable region. This is especially important because susceptibility to progressive multiple sclerosis in DRw15+ patients may be associated with the variable region of the TcR $\beta$ chain locus, but these findings have not been universally reproducible.
Methods and Results: The hypothesis was tested using a contingency table test of patient data and affected family-based controls, control alleles and haplotypes made up of 1) parental marker alleles and haplotypes not transmitted to the affected child from 61 simplex families and 2) alleles and haplotypes not transmitted to the affected child from the unaffected parent in 20 multiplex families collected from British Columbia. Approximately 3,300 alleles at markers spanning the TcR $\beta$ chain complex were segregated through the families containing probands with the RP or RR form of the disease. The VB8-Bam $\mathrm{H} 1$ and V $\beta 11-\mathrm{Bam} \mathrm{H} 1$ allele frequencies $(\mathrm{p}=0.035$ and 0.01 respectively) in the $D R w 15^{+} \mathrm{RP}$ (but not $R R$ ) MS patients were significantly different from control frequencies. The $V \beta 8-V \beta 11$ subhaplotype frequencies in the DRw15+ RP MS (but not RR) patients $(p=0.028)$ were significantly different from control frequencies due to an increase of the 2-1 subhaplotype $(p=$ 0.008 ). The V $\beta 7-B a m \mathrm{H} 1$ genotype frequencies of the DRw15RP MS patients differed from those of RR patients $(p=0.06)$ due to a significantly increased frequency of the 1,1 genotype ( $p$ $=0.02 ; \mathrm{RR}=8.0$ ).

Conclusions: Susceptibility to RP MS in DRw $15^{+}$patients is associated with a gene located on the 2-1 subhaplotype defined by the V $\beta 8$-Bam $\mathrm{Hl}$ and V $\beta 11-\mathrm{Bam} \mathrm{HI}$ alleles of the TcR $\beta$ chain complex. Inheritance of the V $\beta 7$-Bam $H 1$ 1,1 genotype may confer a relative risk of 8:1 to develop RP MS in DRw15 patients.

\section{B-07}

Acetazolamide Responsive Paroxysmal Vestibulocerebellar Ataxia is Genetically Distinct from the Other Episodic Ataxia Disorders

J.L. Steckley, A.F. Hahn, W.J. Koopman, B. von Brederlow, G.C. Ebers, D.E. Bulman (London, Ontario)

Background: Acetazolamide responsive paroxysmal vestibulocerebellar ataxia (APVA) is an autosomal dominant disease characterized by vertigo, nausea, vomiting, and truncal ataxia.

Methods: We have identified two large Canadian kindreds segregating APVA. One pedigree spans three generations, consisting of 51 people, 10 of whom are affected. The second pedigree consists of 186 members, spanning six generations, of which DNA has been obtained for 86 individuals. Short tandem repeat polymorphisms (STRPs) tightly linked to candidate disease gene regions were analyzed with two-point linkage analysis using version 5.1 of the LINKAGE computer programs.

Results: Though APVA is currently included with the autosomal dominant paroxysmal ataxias under a single diagnostic heading (MIM: 108500), the analysis of STRPs in both pedigrees reveals that APVA is genetically distinct from acetazolamide responsive hereditary paroxysmal cerebellar ataxia (APCA), mapped to chromosome 19p. We have also excluded APVA from loci linked to episodic ataxia with myokymia on chromosome $12 \mathrm{p}$. A voltage gated sodium channel gene on chromosome $12 q 13$, SCN8A, that has been suggested as a candidate gene for inherited episodic neurodegenerative disease has also been excluded from APVA. 
Conclusions: APVA is genetically distinct from other episodic autosomal dominant ataxias which have been linked. We are currently conducting a genome wide screen in order to map the APVA gene.

\section{B-08}

Localization of the Gene for Acetazolamide Responsive Paroxysmal Cerebellar Ataxia to a 4.5 Mb Region of Chromosome 19

D.E. Bulman, B. von Brederlow, A.F. Hahn, W.J. Koopman, G.C. Ebers (London, Ontario)

Background: Acetazolamide responsive hereditary paroxysmal cerebellar ataxia (APCA) is a rare autosomal dominant disorder characterized by attacks of cerebellar ataxia and dysarthria with normal or near normal neurologic function between attacks.

Methods: A genome-wide search using polymorphic di- and tri-nucleotide repeats was initiated and the APCA locus was found to be linked to the short arm of chromosome 19 in two large kindreds.

Results: The microsatellite marker UT705 was found to be linked to the APCA locus with two point analysis yielding a maximum lod score of 8.20 at $\theta_{\max }=0.000$ in a 5 generation pedigree. Linkage to this region was confirmed in a second kindred. The cumulative lod score was 10.57 at $\theta_{\max }=0.000$. A physical map of the APCA region spans approximately $4.5 \mathrm{Mb}$ of chromosome 19p13.2. This is the same region of chromosome 19 where familial hemiplegic migraine (FMH) has been mapped.

Conclusions: We are actively searching for additional families with this disorder in order to further refine the position of the gene. In addition, we are currently screening a yeast artificial chromosome contig of this region for expressed sequences in order to identify candidate genes. Both APCA and FHM patients will be screened for mutations.

\section{B-09}

Familial Muscle Hypertrophy with Cramps. A Novel Disorder Linked to the Ryanodine Receptor

\section{M.J. Strong, D.E. Bulman (London, Ontario)}

Background: We have observed the occurrence of muscle hypertrophy with cramps in four generations of a single family.

Methods: A 46 year old female presented with a 5 year history of muscle hypertrophy and cramps that onset asymmetrically in the lower limbs and progressed to involve all limbs, the intercostal musculature and facial muscles. Cramps were induced by exertion, but not by cold, glucose or alcohol exposure. Between episodes of cramping, a low grade myalgia persisted. Strength was normal on initial exertion, but fatigued thereafter. An increase in bulk of all muscle groups was observed without fasciculations or myotonia. Extraocular movements and deep tendon reflexes were normal. The cramps were virtually abolished by dantrolene but not by quinine, tocainamide, verapamil, alpra- zolam and mexilitene. A son, aged 24 , had prominent calf muscles as an infant and thereafter generalized muscle hypertrophy. His daughter has had prominent lower limb musculature from birth. The proband's mother, aged 76, has suffered long-standing calf hypertrophy with cramping.

Results: Creatine kinase levels have been repeatedly normal in the proband, as have been EMG studies during episodes of cramping. A modified barium swallow, obtained for dysphagia, showed a delay in the initiation of pharyngeal peristalsis. Muscle biopsies from clinically involved muscles were normal, including routine histochemistry, electron microscopy, myophosphorylase and mitochondrial staining. Topical NMR spectroscopy demonstrated a prolonged post-exercise acidosis. Linkage analysis is consistent with this disorder being linked to the ryanodine receptor. Further affected members are being studied.

Conclusions: Familial muscle hypertrophy with cramps is a novel autosomal dominant neuromuscular disorder with dantrolene-responsive muscle cramps and linkage to the ryanodine receptor.

\section{B-10}

\section{A New Familial Syndrome: Congenital Cataract with Late Onset Dementia and Psychosis}

\section{A.J. Hudson AND D.G. Munoz, (London, Ontario)}

Background: Two generations of a French-Canadian family with cataract and late onset dementia/psychosis are described. However, only a single member of the first generation appears to have had the disorder whereas 6 of 11 of her progeny were affected. Hence, the disorder is probably inherited as autosomal recessive through a consanguineous marriage. Additional to blindness and dementia some affected members had, to different degrees, mild to moderate mental retardation, dysphagia, movement disorder (ranging from tremor to choreiform movements) and irritable bowel.

Results: The pathological findings were restricted to the central nervous system and consisted of microencephaly (brain weight $980 \mathrm{~g}$ ), and selective severe atrophy of the hippocampal dentate gyri. The remaining dentate granular cells expressed cytoplasmic ubiquitin, but no argyrophilic deposits of any kind were present anywhere in the brain. Additional findings consisted of rare neostriatal bullous neurons, resembling Opalski cells, and mild degeneration of the substantia nigra associated with neither Lewy bodies nor neurofibrillary tangles. The microencephaly must be attributed to hypoplasia, since there was no evidence of cortical or basal ganglionic gliosis.

Conclusions: The association of hereditary congenital cataracts, cerebral hypoplasia and selective degeneration of the dentate constitute a unique, previously undescribed disease entity, which raises the possibility of alterations in proteins shared by brain and lens, such as the crystallins. 


\section{B-11}

Adult Niemann - Pick Disease with Cerebellar Degeneration, Dementia, Polyneuropathy and Normal Cholesterol Metabolism

R.H. WILSON, (Scarborough, Ontario), J. BilbaO (Toronto, Ontario)

Background: The development of knowledge about the cause of Niemann - Pick disease has evolved by first identifying different clinical varieties of this condition and then attempting to define their specific metabolic defect.

Method: A description of a brother and sister with hepatosplenomegaly who developed a global cerebellar disturbance in their 2nd decade and were followed for 15 years. Their sphingomyelin level, sphingomyelinase activity and cholesterol ester formation were tested using cultured skin fibroblasts. MRI of the brain, nerve conduction tests and sural nerve biopsies were performed.

Results: There was an accumulation of sphingomyelin, the sphingomyelinase activity was absent, and cholesterol ester formation was normal. Both patients had a severe demyelinating neuropathy and severe generalized cerebellar atrophy. The sister is in her 6 th decade. The brother died in his 5th decade.

Conclusion: The purpose of this paper is to expand the clinical knowledge of the adult form of Niemann - Pick disease. These two cases had a severe demyelinating polyneuropathy not described in present classifications. The absence of a supranuclear gaze palsy, the normal cholesterol metabolism and the more prolonged course would suggest that this is a separate subtype of adult Niemann - Pick disease.

\section{PEDIATRIC NEUROLOGY}

\section{C-01}

Neonatal Sinovenous Thrombosis and Arterial Ischemic Stroke: Prospective Study of Clinical and Radiographic Features

G.A. DEVeber, M. AdAms (Hamilton, Ontario) on behalf of the Canadian Pediatric Ischemic Stroke Study Group

Background: Prospective study of large numbers of neonates with sinovenous thrombosis (SVT) and arterial ischemic stroke (AIS) is necessary in order to establish the clinical and radiographic spectrum of these conditions. Multicentre collaborative research enables this type of study.

Methods: From Jan. 1 '92 to Dec. 1 '95 we prospectively identified 61 term neonates ( $<1 \mathrm{mo}$. age) with radiographically proven SVT $(\mathrm{N}=22)$ or AIS $(\mathrm{N}=39)$ in the Canadian Pediatric Ischemic Stroke Registry ( $\mathrm{N}=16$ centres).

Results: Neonates represented $45 \%$ of SVT and 17\% of AIS pediatric patients $\leqslant 18$ yrs. Gender involvement was nearly equal. In AIS: $\mathrm{M}=49 \%, \mathrm{~F}=51 \%$; in SVT: $\mathrm{M}=59 \%, \mathrm{~F}=41 \%$. AIS's involved large vessel, left hemisphere in $17(44 \%)$; large vessel, right in $13(33 \%)$; small vessel, right in $7(18 \%)$ and small vessel, left in $4(10 \%)$ and. were hemorrhagic in $33 \%$. In
SVT, parenchymal lesions were present in $45 \%$ (hemorrhagic in 9 of 10). Clinical presentations in AIS were: seizures (77\%), lethargy (13\%) and hemiparesis (10\%). In SVT they were seizures $(68 \%)$, apnea and lethargy $(9 \%)$, with nonspecific signs in the remainder $(24 \%)$. Potential risk factors in AIS were: none definable $(38 \%)$, complicated birth (15\%), sepsis (15\%), patent foramen ovale $(13 \%)$, cardiac sugery, asphyxia and coagulopathy (5\% each) and dehydration (3\%). Risk factors in SVT were: none definable $(36 \%)$, dehydration $(55 \%)$, coagulation disorder and sepsis ( $5 \%$ each). Follow-up was 1 month to 2.5 yrs (mean 9 mos.). In AIS 3 infants received heparin and all had normal outcome: of the 35 patients without anticoagulants, 7 (20\%) had seizure disorder, $12(34 \%)$ had neurological deficits and 16 (46\%) were normal. In SVT 9 patients received heparin: 8 had normal outcome and 1 had neurological deficit. In the remaining 13, 11 were normal, 1 had neurological deficit and 1 died.

Conclusions: Compared to older infants and children, neonates are at high risk of AIS and especially SVT and clinical presentation is relatively non-specific. Further longitudinal study and clinical trials are necessary in order to define outcome and optimal therapy. (Funded by Bloorview Children's Hospital Foundation.)

\section{C-02}

Mild Hypothermia and Amino Acid (AA) Efflux During Hypoxia-Ischemia (H-I) in the Immature Rat

\section{J.Y. YAGER, E. ARMSTRONG, (Saskatoon, Saskatchewan)}

Background: We have previously shown that the neuroprotective effect of mild hypothermia is only partially explained by a preservation of high energy phosphates. At $31^{\circ} \mathrm{C}$ ATP is completely preserved whereas ATP is equally depleted by $80 \%$ at 34 and $37^{\circ} \mathrm{C}$. To further investigate the mechanisms underlying the protective effect of mild hypothermia, in vivo microdialysis was utilized to determine hippocampal amino acid (AA) efflux during $\mathrm{H}-\mathrm{I}$ and the results correlated with the extent of brain injury.

Methods: 7-day rats underwent unilateral common carotid artery ligation and exposure to $8 \%$ oxygen for 3 hours at either 31,34 , or $37^{\circ} \mathrm{C}$. Microdialysis probes (CMA 11) were placed in the ipsilateral $\mathrm{CA}_{1}$ region of the hippocampus, and dialysate collected for $20 \mathrm{~min}$. at $1 \mathrm{hr}$ prior to, and at 60,120 , and 180 min during H-I. Samples were analyzed for alanine, glutamine, glutamate, glycine, taurine, serine, and GABA by high-performance liquid chromatography. Rat pups were recovered to 30 days of age for subsequent neuropathologic analysis. Serial coronal slices of each brain were made and percent volume of brain damage made by computer image analysis.

Results: No differences in extracellular AA concentrations were noted prior to $\mathrm{H}-\mathrm{I}$. During $\mathrm{H}-\mathrm{I}$, concentrations of all AA in the $37^{\circ} \mathrm{C}$ group, except glutamine, rose significantly above control values $(p<0.001)$. In the $34^{\circ} \mathrm{C}$ group, only alanine increased above control $(p<0.01)$, whereas the other AA remained within control limits. No increases in AA levels were seen in the $31^{\circ} \mathrm{C}$ group. A direct correlation existed between the \% brain damage and degree of AA efflux, except glutamine ( $p<0.001)$.

Conclusions: Thus, 1) The mechanism of protection by mild 
hypothermia is temperature dependent, 2) Mild hypothermia of $34^{\circ} \mathrm{C}$ exerts its protective effect by suppressing excitatory AA release, and 3) The extent of AA efflux is predictive of $\%$ brain damage.

\section{C-03}

\section{Predicting Neurodevelopmental Outcome from Head Ultrasound in Preterm Infants}

M. KLOTH (Kingston, Ontario) S.D. LEvIN, W.B. Dawson, M.H. KIM, D. Louw (London, Ontario)

Background: Over 50 papers relating head ultrasound scan (HUS) appearance to neurodevelopmental outcome have been published in the last 15 years but data are unreliable.

Methods: In this prospective historical cohort study the HUS of all infants. $1500 \mathrm{~g}$ admitted to our NICU between 1 Jan 1991 and 31 May 1992 were reviewed independently by a paediatric neurologist and radiologist both blind to outcome. Outcome was then predicted. Neurodevelopmental outcome was determined by the neonatal follow-up team. Motor, cognitive, visual, hearing, speech and language domains were assessed at 3 years.

Results: $216 / 255$ infants had HUS. 37 died before HUS could be performed. 21 died before discharge leaving 196 cases. 13 were lost to follow-up. 145/216 (67.1\%) infants had normal HUS. Of these $20.5 \%$ had a motor disorder, $17.6 \%$ had cognitive impairment, 17.5 had visual, $5.8 \%$ had hearing and $32.0 \%$ had speech and language impairment. Apart from motor disorder, IVH alone did not predict increased risk whereas parenchymal lesions were associated with cognitive impairment in $50 \%$ and motor disorder in $80 \% .3 / 15$ infants with PVL had no motor disorder and $5 / 15$ were cognitively normal. The positive predictive value (PPV) for motor disorder was $80 \%$ and negative predictive value (NPV) 75.3\%; for moderate or severe cognitive impairment PPV was $47 \%$ and NPV 97\%.

Conclusion: This study provides useful data about the value of HUS in predicting neurodevelopmental outcome, particularly, the relatively high incidence of problems in those with normal HUS and normal outcome in those with apparently significant HUS lesions.

\section{C-04}

Clinical Importance of Placental Abnormalities Associated with Cystic Periventricular Leukomalacia

E. Rodriguez, J.F. Magee, E.H. Roland, B.A. Lupton, A. HILL, (Vancouver, British Columbia)

Periventricular leukomalacia (PVL) is a pattern of cerebral injury which occurs in premature infants. Although it is considered to result principally from ischemic insult to vulnerable periventricular white matter, other factors, eg., bacterial endotoxins, may have a causative role. In this study, we describe placental abnormalities associated with cystic PVL to identify possible prenatal pathogenetic factors. Clinical data and detailed placental histopathology were reviewed on 50 premature newborns (birthweight $<1500 \mathrm{~g}$ ) who developed cystic PVL.
The incidence of placental abnormalities was higher than expected from clinical and historical data. There was a high incidence of inflammation involving chorion/amnion (73\%), umbilical cord (49\%), and especially fetal surface vessels (43\%). Possible chronic hypoxic changes (eg, villous infarction and advanced maturation, decidual necrosis) were observed in $20 \%$. Acute hypoxic changes included meconium staining (12\%) and intravillous hemorrhage/edema (36\%). No case had fetal surface vessel thrombosis.

These data demonstrate a high incidence of placental abnormalities (eg.,inflammation, acute and chronic hypoxic changes) associated with cystic PVL. Such observations may provide important insights into the pathogenesis of PVL and timing of insult to periventricular white matter.

\section{C-05}

\section{D-2-Hydroxyglutaric Aciduria: A Cause of Neonatal} Metabolic Encephalopathy with Facial Dysmorphism

\author{
K.A. Selby, B. H.Bjornson, H. Vallance (Vancouver, British \\ Columbia)
}

D-2-hydroxyglutaric aciduria has been reported in six patients with variable phenotypes. The enyzme defect remains unidentified. It appears to be of autosomal recessive inheritance, no specific treatments are known, but prenatal diagnosis is possible. We report a neonatal case.

A male child was born at term to non-consanguinous parents following an uneventful pregnancy. He presented in the neonatal period with intractable multifocal seizures, poor visual attention, altered tone and dysmorphic facial features. He had hypoplastic optic nerves and coarctation of the aorta. There were no neurocutaneous findings, no organomegaly and his head circumference was on the fiftieth centile.

Investigations revealed markedly elevated levels of D-2 hydroxyglutaric acid in the urine, plasma and cerebrospinal fluid.There was hypoglycorrhachia despite normoglycemia; CSF protein and GABA were elevated. Magnetic resonance imaging of the brain at 6 months demonstrated generalized decrease in white matter, and the electroencephalogram contained multifocal sharp waves and spikes.

This case broadens the spectrum of the clinical and laboratory findings of this disorder. The hypoglycorrhachia suggests the possibility of a glucose transport defect. This disorder should enter the differential diagnosis of neonatal encephalopathy with dysmorphism.

\section{C-06}

EEG and Long Term Outcome for Full Term Infants with Perinatal Asphyxia

D.B. Sinclair, P. Byrne, W. Prasertsom, C. Robertson (Edmonton, Alberta)

Background: The prognostic value of the Electroencephalograph (EEG) in the prediction of long term outcome for full term newborns is well established. A burst suppression pattern on the EEG in 
term infants who have had evidence of perinatal asphyxia is shown to be predictive of a grave prognosis.

Methods: We retrospectively analyzed all records of newborn infants born at the University of Alberta Hospital between January 1, 1991, and December 31, 1992, with a gestational age of 37 weeks or greater who had evidence of Hypoxic Ischemic Encephalopathy and had at least one EEG during the first week of life. The EEGs were reviewed and subclassified into a Burst Suppression Pattern (BSP), or if the pattern was not continuous or was incomplete, a Modified Burst Suppression Pattern (MBSP) based on specified electrophysiological criteria. The neurological outcome was then correlated with the EEG pattern.

Results: Twenty-three full term infants were studied. Fifteen had a BSP on EEG and 8 had a MBSP. Six of 15 infants with a BSP died. Of the 9 survivors with a BSP, 6 are disabled, 1 is mildly disabled, and 2 are normal. In the MBSP group (8), 1 infant died, 2 were disabled, 1 was mildly disabled and 3 were normal. In the BSP group 6/9 developed cerebral palsy while in the MBSP group only 1/7 developed cerebral palsy. Only 2/9 infants with the BSP were normal while $5 / 7$ patients with the MBSP were normal.

Conclusions: The results are suggestive of a better outcome for patients with the MBSP. Subclassification of the EEG changes of Hypoxic Ischemic Encephalopathy into Burst Suppression and Modified Burst Suppression Patterns may give a more accurate prediction of outcome in perinatal asphyxia.

\section{C-07}

\section{The Clinical Course of Nova Scotia Niemann-Pick Disease}

\section{JAN, P. CAMFIELd (Halifax, Nova Scotia)}

Background: Niemann-Pick type IIS (type D) can be traced to a single Acadian ancestor in western Nova Scotia. We outline the clinical course of this devastating disease.

Method: Children with Nova Scotia Niemann-Pick were identified from multiple sources. Some were our patients. Information about others was completed during 9 structured telephone family interviews.

Results: 20 patients from 15 families were identified. Five children had severe neonatal jaundice. Most developed normally until age 5 years. Cognitive problems appeared in the first 2 school years. Vertical opthalmoplegia was then present, if assessed. By age 6-8 years, clumsiness and frequent falls were prominent. Hand use was lost by 12 years (9-20 years). Unexplained easy bruising and nose bleeds occurred in 7. All developed epilepsy between ages 4.5 - 16 years with subsequent marked deterioration. By age 12 years, $61 \%$ had no expressive speech although some receptive speech and alertness persisted. Incontinence, severe feeding problems and loss of walking occurred about age 10-12. Average age at death was 14.8 years (range 12-22 years).

Conclusions: Despite a uniform genetic cause, there was considerable variability in the clinical course of children with Nova Scotia Niemann Pick. Care for these children was complex and long lasting.

\section{C-08}

Therapeutic Electrical Stimulation (TES) Following Selective Posterior Rhizotomy in Children with Spastic Diplegia due to Cerebral Palsy: a Randomized Clinical Trial

\section{P. Steinbok, A. Reiner, J.R.W. Kestle (Vancouver, British} Columbia)

Therapeutic electrical stimulation (TES), which involves low intensity electrical stimulation applied for many hours daily during sleep, has been introduced recently, with promising results reported in a number of motor disorders in adults and children.

Methods: Randomized controlled trial was carried out to determine the effectiveness of TES in improving the function of children with spastic cerebral palsy, who had stabilized after a previous selective posterior lumbosacral rhizotomy. Children were randomized either to receive TES for one year or to have no TES. The primary outcome was the change in the Gross Motor Function Measure (GMFM), a quantitative and validated measure for use in children with spastic cerebral palsy.

Results: There was a statistically significant and clinically important improvement in outcome for the treated children, with the mean change in the GMFM score at one year being $5.5 \%$ compared to $1.9 \%$ in the untreated group $(p=0.001)$. The difference in means of $3.6 \%$ was clinically important. TES was simple to use, had no significant complications, and was well accepted by children with spastic cerebral palsy and their caregivers.

Conclusion: The positive results of this study open the way for the use of TES in children with spastic cerebral palsy.

\section{C-09}

Monotherapy Clobazam vs Carbamazapine vs Phenytoin in Childhood Epilepsy: A Double Blind Randomized Trial with 220 Canadian Children.

The Canadian Clobazam Study Group for ChIldhood Epilepsy

Background: There have been no previous, large, doubleblind trials to identify the monotherapy antiepileptic drug of choice for childhood epilepsy. Standard therapy has included carbamazepine and phenytoin. Clobazam has seen increasing use based on its wide spectrum of efficacy and low toxicity. The collegiality of Canadian child neurologists has permitted a definitive trial with these 3 agents.

Method: Children aged $2-16$ years with newly diagnosed epilepsy or previous efficacy failure of one drug were assigned to one of two arms and then randomized - clobazam vs carbamazepine or clobazam vs phenytoin. Seizure types included all partial seizures and generalized tonic clonic without myoclonus or absence. Blinding used a "double dummy" technique with blinded serum levels. The primary endpoint was length of retention on medication during the year after randomization.

Results: 14 centres entered 235 patients. Overall, 55\% for clobazam, $57 \%$ for standard therapy. Complete seizure control for the last 9 study months was achieved in 55\% of children receiving clobazam and $58 \%$ on standard therapy. Side effects of the 3 drugs were similar. 
Conclusions: Monotherapy clobazam is equally effective as standard therapy (carbamazepine and phenytoin) for early treatment of childhood epilepsy.

\section{C-10}

\section{Petit Mal with Interictal Minor Focal Features - Natural History}

\section{H. DaRwish, V. Sherwood, V. LANGE (Calgary, Alberta)}

Background: Primary generalized absence seizures (Petit Mal) are identified with an ictal EEG pattern of generalized serial spike and wave discharge, and a normal interictal EEG. In 1984 (CCNS) we defined a group of 30 children whose predominant seizures were absence (with rare generalized tonic clonic convulsions in 11) whose ictal EEG showed serial spike and wave discharge but whose interictal EEG demonstrated focal spikes $(53 \%)$ or slowing (47\%). At the 1987 (AES) we reported a greater efficacy for Valproic acid compared to ethosuximide in this group and showed those with clinically complex absences needed significantly higher serum levels.

Methods: Prospective clinical study of epidemiology. We now test the hypothesis that the minor focal features in the interictal EEG identify a poorer long-term outcome than is usually given for Petit Mal.

Results: 26 children were available for review at a mean age of $19.8 \pm 5.5$ years. 12 are male and 14 are female. The mean age of onset of their absence seizures was $7.9 \pm 2.7$ years. 12 children are currently $6.7 \pm 3.3$ years off medication and seizure-free. The other 14 children have not outgrown their epilepsy at their current mean age of $21.5 \pm 4.7$ years though they have been followed for $13.5 \pm 3.4$ years from onset of their seizures. $5 / 14$ never achieved one year seizure freedom but $7 / 14$ were seizure-free for 2 or more years in their teens and 4 had been withdrawn off medication before recurrence of absences and convulsions (in 6) necessitated reintroduction of medication.

Conclusion: In this group of Petit Mal with minor focal features the outcome is not as good as traditionally considered for Petit Mal. We were also unable to find specific discriminators to define who will outgrow the epilepsy.

\section{C-11}

\section{Vigabatrin and the developing brain}

S.S. Seshia, R. Sidhu, U.I. Tuor, M.R. Del. Bigio (Winnipeg, Manitoba)

Vigabatrin (VGB) is an effective add-on anti-convulsant for a variety of seizure types in children and is now considered to be the preferred drug of choice for the treatment of infantile spasms (Appleton \& Montiel - Viasca 1993, and others). Chronic administration of VGB produces "reversible micro-vacuolation" (intramyelinic edema) in the brains of a variety of adult animals. To our knowledge, there is no information on the effect on the developing brain. Twelve day old rats were treated (SC) with VGB $(\mathrm{N}=8 ; 15$ $200 \mathrm{mg} / \mathrm{kg} / \mathrm{day}$, but most at $40 \mathrm{mg} / \mathrm{kg} / \mathrm{day})$ or saline $(\mathrm{N}=6 ; 0.1$ $\mathrm{ml} / 10 \mathrm{~g}$ ), for 5 days. They were perfusion fixed after 2 days of recovery. The neuropathological changes were as follows: (i) densitometric analysis of Luxol Fast Blue stained sections showed statistically significant reduction of myelin in the external capsule and middle cerebellar peduncles in treated animals but not in controls, (ii) electron microscopy showed scattered degenerating axons in the corpus callosum and external capsule of $4 / 4$ treated animals but $0 / 4$ controls, (iii) reactive astrogliosis was evident in the frontal lobe grey matter of $5 / 8$ treated animals but in $0 / 6$ controls. These findings suggest that VGB, at doses suggested for children, has adverse effects, some of which are potentially irreversible, on the developing (rat) brain. Further animal and clinical studies are warranted.

\section{SURGICAL ONCOLOGY}

\section{D-01}

Human Brain Tumor Cell Spreading and Migration on Central Nervous System(CNS) Myelin Correlates with the Grade of Malignancy.

V. Amberger, R. Del Maestro (London, Ontario) Y. Yonekawa, M. Schwab (Zurich, Switzerland)

Background: Highly malignant brain tumors infiltrate the surrounding tissue and migrate preferentially on white matter fiber tracts. CNS myelin, however, contains two inhibitory proteins, Neurite Growth Inhibitors NI 35/250, which prevent neurite outgrowth and cell spreading of many different cell types, e.g. astrocytes and fibroblasts.

Methods: Specimens from brain tumor patients were maintained in short term culture (7-9 days) and subsequently tested for their ability to spread and migrate on purified rat myelin extracts.

Results: Cells taken from benign or low grade tumors attached poorly to and did not spread or migrate on the substrate. Whereas cells from highly malignant tumors attached and spread on the CNS myelin without difficulty. In the presence of a metalloprotease blockers, like o-phenanthroline or cbzPhe-Ala-Phe-Tyramide spreading of highly malignant tumor cells was inhibited.

Conclusion: The ability to spread and migrate on CNS myelin correlates with the increasing grade of malignancy of the original tumor. Highly malignant brain tumors express a metalloprotease which enables their cells to overcome the inhibitory effect of the NI-35/250 present in white matter. The possibility of selective blockade of tumor cell infiltration by metalloprotease inhibitors opens new possibilities for the treatment of malignant brain tumors.

\section{D-02}

Preliminary Models of Ex Vivo Immunogene Therapy for Glioblastoma Multiforme

I.F. Parney, K.C. Petruk, M. Farr-Jones, L.J. Chang (Edmonton, Alberta)

Background: The dismal prognosis of glioblastoma has led to investigation of novel treatment strategies such as gene therapy. 
We have developed a model for ex vivo immunogene therapy for glioblastoma using genes for the T-cell costimulatory molecule B7-2 and the cytokine GM-CSF.

Methods: GM-CSF and B7-2 genes were cloned into retroviral vectors and transfected into the human glioblastoma cell line D54MG. Gene expression was characterized by ELISA and flow cytometry. Transduced D54MG cells were injected subcutaneously in SCID/bg (Severe Combined Immunodeficiency) mice reconstituted with allogeneic human lymphocytes. Tumor growth was observed. Some mice were challenged with wild type D54MG cells. Splenocytes were isolated for proliferation and cytotoxicity assays vs. wild type D54MG.

Results: Transduced D54MG cells expressed GM-CSF and B7-2 well. Growth inhibition was moderate for GM-CSF and marked for B7-2 expressing tumors. Presence of GM-CSF or B7-2 transduced tumor inhibited growth of wild type D54MG challenges. Splenocytes from mice harboring transduced tumors showed increased proliferation and cytotoxicity when challenged with wild type tumor.

Conclusions: This study shows ex vivo immunogene therapy for glioblastoma is feasible. Initial results show promising efficacy. Further aspects of this model need to be developed.

\section{D-03}

Basic Fibroblast Growth Factor and Vascular Endothelial Growth Factor Down-regulate Collagenase Type IV Activity of C6 Astrocytoma Cells in Monolayer Culture.

M. Tamaki, W. McDonald, R.F. Del Maestro (London, Ontario)

Background: Tumor associated angiogenesis is complex and a number of enzymatic activities, angiogenic factors and extracellular matrix (ECM) molecules are involved in this phenomenon. We have investigated the effects of basic fibroblast growth factor (FGF-2) and vascular endothelial growth factor(VEGF) on collagenase IV activity (Gelatinase $A$ and extracellular proteasome) and production of ECM using C6 astrocytoma cells in vitro.

Methods: FGF-2, VEGF and the ECM molecules such as laminin and fibronectin were added to $\mathrm{C} 6$ astrocytoma cells in monolayer culture (serum free media). Collagenase IV activity was assayed biochemically and by Western blot analysis. The production of ECM in the conditioned medium was investigated using Western blots.

Results: (1) Gelatinase A and extracellular proteasome activities were decreased by the growth factors tested. (2) The growth factors assayed increased the production of extracellular laminin and fibronectin. (3) Addition of exogenous laminin and fibronectin to the medium decreased collagenase IV activity.

Conclusion: These findings suggest that the growth factors tested may be involved in regulatory systems for the production of collagenase IV. Namely growth factors such as FGF-2 and VEGF may modulate collagenase IV activity via their increasing effect on laminin and/or fibronectin production. These may be important to allow a co-ordinated angiogenic response to a tumor stimulus.

\section{D-04}

Expression of Vascular Permeability Factor/Vascular Endothelial Growth Factor(VPF/VEGF) in Meningiomas and Assosciated Peritumoral Edema

\section{J. Provias, K. Claffey, A. Guha, (Toronto, Ontario)}

VPF or VEGF is a highly secreted mitogenic growth factor, which acts specifically on vascular endothelial cells. High affinity receptors of VPF/VEGF (KDR \& FLT-1) are expressed only on endothelial cells. VPF/VEGF has been shown to be present in a number of solid tumours and to play a prominent role in tumour associated angiogenesis and the development of tumour related edema. The role of VPF/VEGF in the central nervous system has been studied in astrocytic tumours but not in meningiomas, which are the second most common primary brain tumor. These tumors although benign can be highly vascular and sometimes associated with marked peri-tumoral edema, both features potentially linked to VPF/VEGF. In this study expression of VPF/VEGF and its receptors, were examined in meningioma specimens.

Northern analysis, in-situ hybridization and immunohistochemistry demonstrated that VPF/VEGF was expressed in majority of meningiomas. Immunohistochemistry localized VPF/VEGF to meningothelial tumour cells but not the vascular endothelial cells. In-situ hybridization demonstrated that the receptors (KDR \& FLT-1) were expressed only in endothelial cells within the tumor. In some tumours,there were subtle areas of endothelial hyperplasia, with VEGF expression in the meningothelial cells around these vessels. VPF/VEGF mRNA expression was upregulated in meningiomas associated with prominent peritumoral edema, with the reactive astrocytes found in these edematous areas staining for VPF/VEGF. This study demonstrates that VPF/VEGF and its receptors are expressed in meningiomas, and potentially contribute to the angiogenesis and assosciated peri-tumoral edema in these tumors.

\section{D-05}

The Incidence of Silent Hemorrhage After Stereotactic Biopsy

A.V. Kulkarni, A. Guha, A. Lozano, and M. Bernstein (Toronto, Ontario)

Background: Many neurosurgeons routinely obtain CT scans after stereotactic biopsy to rule out unsuspected hemorrhage. The present study investigated the efficacy of this practice and determined the rate of silent, unsuspected hemorrhage after stereotactic biopsy.

Methods: All patients undergoing a frame-based stereotactic biopsy at the Toronto Hospital between June 1994 and December 1995 had a CT scan done within hours of the procedure. The medical records and CT scans were then reviewed retrospectively by the authors.

Results: Seventy-one patients (ages 17-87 years) underwent stereotactic biopsies and had post-operative CT scans between June 1994 and December 1995. Forty-three (60.6\%) patients 
had hemorrhages on the immediate post-operative scan, the vast majority of which were intra-parenchymal. Only 4 of these patients demonstrated new neurologic deficit in the immediate post-operative period and in the remaining $39 / 71$ (54.9\%) the hemorrhage was clinically silent and unsuspected. Amongst the clinically silent intra-cerebral hemorrhages 16 were $<5 \mathrm{~mm}, 16$ between $5-10 \mathrm{~mm}, 2$ between $10-20 \mathrm{~mm}$, and 3 between $30-$ $40 \mathrm{~mm}$. Although the rate of silent hemorrhage was surprisingly large, in no case did the finding of an unsuspected hemorrhage beneficially affect patient management. Of the 39 patients with clinically silent hemorrhages only 2 demonstrated a delayed neurologic deficit ( 1 seizure and 1 progressive loss of consciousness) and these both occurred on the first post-operative day.

Conclusions: The incidence of silent hemorrhage after stereotactic biopsy was found to be $54.9 \%$, but rarely did a clinically silent hemorrhage ultimately affect patient management. We therefore do not feel that a routine post-operative CT scan after uncomplicated stereotactic brain biopsy is warranted in the majority of patients.

\section{D-06}

Frequency of Follow-up MRI Scanning in Patients with Malignant Glioma - Will Less Frequent Scanning Jeopardize Our Patients?

\section{FULTON (Edmonton, Alberta)}

Background: Follow-up of patients with primary malignant brain tumor, intended to identify response, recurrence and side effects of therapy, involves repeated neuro exams and CT or MRI. The neuro exam alone does not exclude recurrence. Early detection of recurrence allows therapy before neurological deterioration and may improve quality of life. It is not known whether or not early detection and treatment of recurrence prolongs time to tumor progression (TTP) or survival time (ST). Recent budget cuts have resulted in the need to reduce the frequency of routine follow-up scans in patient with brain tumor.

Methods: We reviewed the medical records of 89 consecutive patients treated with chemotherapy plus or minus surgery for their first recurrence of supratentorial malignant glioma. Initial treatment consisted of surgery followed by radiation therapy \pm adjuvant chemotherapy. Initially, patients routinely had CT or MRI scans scheduled prior to each dose of chemotherapy (6 to 8 week interval). Later, because of budget cuts, scans were scheduled prior to alternate doses of chemotherapy (12 to 16 week interval). Scans were done early for signs or symptoms of recurrence.

Results: Median TTP (MTTP) in weeks from date of progression was 20.9 for all patients, 19.4 for those scanned prior to each chemo and 25.7 for those scanned prior to alternate chemo. Median ST (MST) in weeks was 46.1 for all patients, 52.1 for those scanned prior to each chemo and 40.4 for those scanned prior to alternate chemo. Median TTP (MTTP) in weeks from date of progression was 16.3 for patients with glioblastoma multiforme, 12.1 for those scanned prior to each chemo and 25.7 for those scanned prior to alternate chemo. Median ST (MST) in weeks was 32.3 for all patients, 27.1 for those scanned prior to each chemo and 35.5 for those scanned prior to alternate chemo.

Conclusions: Differences in MTTP and MST can be accounted for by lead time bias. There is no evidence that less frequent scanning is detrimental. A prospective study including quality of life measurement is planned.

\section{E. CEREBROVASCULAR SURGERY}

\section{E-01}

Surgery versus Stereotactic Radiation for Small, Operable Brain Arteriovenous Malformations: A Decision Analysis

\section{P.J. PoRTer, M.C. Wallace, A.S. Detsky (Toronto, Ontario)}

Background: Brain arteriovenous malformations (AVMs) afflict young patients with potentially devastating effects. For small $(<3 \mathrm{~cm})$, surgically accessible AVMs, the major treatment options are surgery and stereotactic radiation (SR). It is not known which of these strategies is preferable. The major tradeoff between them hinges on the fact that outcomes, both favourable and unfavourable, occur immediately with surgery but are delayed with radiation.

Methods: Using Smltree ${ }^{\mathrm{R}}$ software, a decision analysis model was developed to address the dilemma of which therapeutic option, surgery or SR, is preferable for small, operable AVMs. The major factors in the model are the probabilities of cure and complications in both strategies, the probability and sequelae of hemorrhage, and patient preferences related to each procedure. Values for probabilities and utilities were derived from the literature when available. Otherwise, empiric estimates were used. The baseline values used were: Surgery - 95\% cure, $5 \%$ morbidity, $1 \%$ mortality; SR - $80 \%$ cure, $3 \%$ morbidity, and $6 \%$ risk of hemorrhage during the latency period to cure.

Results: For the baseline analysis, surgery is the preferred strategy, confering A 1.1 quality-adjusted life-year advantage over radiation. This result changes only when values at the extreme end of the clinically plausible range are considered for the probability of cure with radiation, and the morbidity and mortality of surgery. The design is limited in that it only considers one-time treatment, and likely cannot fully capture the importance of risk aversion as it relates to surgery.

Conclusions: For small, operable AVMs, surgery is preferred over SR. The reason is that surgery protects earlier and with greater success. However, there is some uncertainty in several key variables in the model. To know which treatment should be offered to such patients, it is essential that centres be aware of their surgical morbidity and mortality, and radiation cure rates.

\section{E-(02}

\section{Functional Imaging in AVM Patients}

R. Leblanc, E. Meyer, R. Zatorre, D. Tampieri, A. Evans (Montreal, Quebec)

Background: The successful resection of cerebral AVMs is largely a function of their size, the number of feeding arteries, 
the complexity of the drainage system, and their proximity to eloquent brain regions. The availability of alternatives to surgery, such as stereotactically-focused radiotherapy, make identification of the relationship of an AVM to functionally important, ambient brain regions, all the more important. Thus, we undertook this series of observations in patients with cerebral AVMs to determine the usefulness of f-PET in establishing the relationship between the lesion and the surrounding cortex in treatment planning.

Methods: We have evaluation f-PET during sensory stimulation or during the performance of auditory, visually-based or expressive language tasks to determine its reliability in establishing the relationship of cerebral AVMs to the ambient cortex in 11 patients. Five patients with a central AVM underwent activation-PET scanning combined with 3-D MRI during vibro-tactile stimulation of the contralateral hand, and 6 patients with AVMs in or about speech areas were similarly scanned during a language task.

Results: Vibro-tactile stimulation identified the post-central gyrus, establishing the relationship of the AVM to the central region. The results of language activation were specific to the modality (visual, auditory, articulate), on which the task was based.

Conclusions: Sensory f-PET is reliable in identifying the preand postcentral gyri and useful in assessing AVMs in and about the central region. A greater understanding of results in normal controls for specific visual, auditory, and expressive language tasks is necessary before language activation f-PET can be confidently applied to clinical cases. Similar conclusions would seem applicable to f-MRI.

\section{E-03}

Arterial Hemodynamic and Anatomic Factors in the Clinical Presentation of Cerebral Arteriovenous Malformations

S.P. Lownie, (London, Ontario), E.F. Raupp, (Porto Alegre, Brazil) G.R. DUCKwILER, F. Viñuela (Los Angeles,California)

Background: Feeder artery pressures have been implicated in hemorrhage from cerebral arteriovenous malformations (AVMs).

Methods: We monitored feeder artery pressures in 45 patients with AVMs (16 presenting with hemorrhage, 29 without) during superselective angiography.

Results: Mean feeder artery pressure (FP) was found to be 49 $\mathrm{mm} \mathrm{Hg}$. The mean FP in patients with hemorrhage was somewhat higher than in those without hemorrhage, but the difference was not statistically significant $(53.8 \mathrm{~mm} \mathrm{Hg}$ vs. $47.0 \mathrm{~mm} \mathrm{Hg}, \mu=0.13$ ). A moderate correlation between FP and AVM nidus size was discovered ( $r=-0.42, \mu=0.0043$ ). Systemic mean pressure was also found to correlate with AVM size ( $r=-0.31, \mu=0.037$ ). Multiple linear regression analysis showed that the significant predictors of FP were systemic pressure, AVM size and the distance of the microcatheter from the circle of Willis. Logistic regression of anatomic and physiologic variables revealed that the presence or absence of venous outflow stenosis and the position of the AVM nidus (superficial or deep to the cortical surface) were the most significant predictors of AVM hemorrhage versus seizures.
Conclusion: It is concluded that anatomic factors are probably more important than arterial hemodynamic factors in determining the clinical presentation of cerebral AVMs.

\section{E-04}

Intracranial Dural Arteriovenous Fistulas: Their Natural History and Management

M.A. Davies, K. TerBrugge, R. Willinsky, M.C. Wallace (Toronto, Ontario)

Our large experience with Intracranial Dural Arteriovenous Fistulas (ICDAVF) defines the natural history of ICDAVF for the first time. The utility of the grading scale of Borden et al (Borden*)as a simple tool for predicting lesion behaviour is assessed. We explore the logic of a conservative approach to benign lesions, the role of embolization, and also for the first time examine the outcome of surgical disconnection of Retrograde Leptomeningeal Venous Drainage (RLVD) alone for management of aggressive ICDAVF.

A series of 102 ICDAVF of all types seen between 1984 and 1995 is examined. Fifty five lesions were Borden* grade 1. During the followup period (mean 32 months), $21 / 26$ (81\%) improved with conservative treatment, compared with $20 / 23$ $(87 \%)$ of the embolized patients respectively $(p=N S$ ).

All grade II and III ICDAVF were considered aggressive lesions and offered treatment. Forty six of these lesions were followed for 1342 lesion months. Conservative therapy was chosen by 14 patients and interval hemorrhage, non hemorrhagic neurological deficit and mortality rates, were observed to be $19.2 \%, 10.9 \%$, and $19.3 \%$ per lesion year respectively. Twenty five surgeries were performed on 23 ICDAVF. Resection of the ICDAVF was performed in 9 patients, and 16 patients were treated with disconnection alone. Complications occured in 3/9 (33\%) of the resected group and none of those who had disconnection alone. Failure to obliterate RLVD in two patients who had resections resulted in adverse outcomes in both patients. All $16(100 \%)$ of the disconnected group were shown to have undergone angiographic obliteration with excellent clinical outcome.

The benign natural history of Borden* grade I ICDAVF, mandates a conservative approach. Untreated, Borden* grade II and III ICDAVF have a poor natural history. In addition, treatment which fails to eliminate RLVD is inadequate and leads to poor outcome. Surgical disconnection alone results in cure of all Borden* grade III ICDAVF, and in grade II lesions, if not cure, conversion to a benign grade I lesion.

\section{E-05}

Incidents at the Very Moment of the Cerebral Aneurysm Rupture

\section{S.H. Leivo J.A. Hernesniemi S.J. Peerless C.G. Drake (Kuopio, Finland)}

The incidents at the very moment of cerebral aneurysm rupture were analyzed in two large series. Only those cases with exactly documented data were included: our scrutiny deals with 
1699 patients from Kuopio, Finland and from London Ontario, Canada.

In Kuopio $44.7 \%$ of the 917 patients had their aneurysm rupture during physical activity, $21.7 \%$ during nonstrenous events and $21.6 \%$ during rest or sleep. The corresponding figures for London, Ontario series (782) were $28.3 \%, 35.9 \%$ and $10 \%$ respectively. Aneurysm rupture during sexual intercourse was nearly twice as frequent in the Canadian series $(4.9 \%$ versus $9.0 \%$ ) and occurred mainly in males in both series. Further, aneursym rupture in emotional situations was extremely rare in the Finnish series, only $1.1 \%$ as compared with $7.8 \%$ in the Canadian series.

Those patients having their aneurysm rupture during sleep had poorer grades on and worse outcomes than those patients having their aneurysmal rupture when awake. No significant associations between circumstances around aneurysmal rupture and aneurysmal sites could be seen in either series.

Traditional medical advice given to patients with ruptured aneurysms (avoidance of heavy lifting or straining, sexual abstinence or recommendations to rest) is likely to be ineffective to prevent aneurysmal rupture. These deadly lesions bleed throughout 24 hours, even during sleep and sedentary activity when awake. Furthermore, worker's compensation claims relating aneurysmal rupture to work are likely to be futile.

\section{E-06}

\section{Endovascular Treatment of Basilar Tip Aneurysms}

J. Raymond, D. Roy, M.J. Bojanowski, R. Moumdian G. L'EsPerance, D. Venne (Montreal, Quebec)

Prospective study of complications, immediate and long term angiographic and clinical results in 31 patients referred for treatment with Guglielmi detachable coils.

There were 18 women and 13 men. Age ranged from 34 to 67 years old (mean 48 ). Twenty-three were treated acutely following subarachnoid hemorrhage. $95 \%$ of patients in grade I to III at presentation had a good outcome (18/19). There were 9 technical complications, most often asymptomatic but 1 patient died after aneurysm perforation and 1 patient was left with diplopia at 6 month follow-up after a stroke (morbidity 3\%; mortality $3 \%$ related to treatment). Immediate angiographic results were satisfactory (complete obliteration or residual neck only) in $93 \%$ of patients. There was no bleeding episode following treatment during clinical follow-up varying from 3 months to 3 years (mean: 15.25 months in 29 surviving patients).

Angiographic results are available in $63 \%$ of patients at 6 months: $88 \%$ of lesions are completely obliterated or present only a residual neck. Angiography at 1 year is available in $37 \%$ of patient, with $90 \%$ satisfactory results. Growing recurrences were noted in 5 patients with large, wide neck aneurysms, necessitating retreatment with GDC in 4 patients 3 months to 2 years after initial treatment.

Endovascular treatment of basilar tip aneurysms prevents rebleeding and could be performed safely in $93 \%$ of patients. Clinical results following SAH compares favorably with surgical series; long term angiographic results appear less satisfactory but retreatment can be easily performed.

\section{E-07}

\section{A Regional Performance of Carotid Endarterectomy}

J.M. Findlay, M.E. Suarez-Almazor, J.H. Wong (Edmonton, Alberta)

Objective: Although randomized trials have established that carotid endarterectomy (CEA) is effective in reducing stroke risk in some patients, its benefit depends upon the accurate determination of angiographic stenosis, correct patient selection, and acceptable complication rates. We wished to assess the appropriateness and safety of CEA as currently practiced in our healthcare region.

Methods: Prospective and retrospective cohort study utilizing hospital chart review of all 292 patients undergoing CEA within an 18 month period in the city of Edmonton. Based upon randomized trial data, patient surgical indications were stratified into appropriate, equivocal, or inappropriate categories. Angiogram films were remeasured in accordance with NASCET guidelines in blinded fashion. Information was obtained from office and hospital charts, telephone interviews, and government registries.

Results: In this series $40 \%$ of operations were for asymptomatic stenosis. Inappropriate indications for surgery were found in $26 \%$ of cases, predominantly due to overestimation of the angiographic degree of carotid stenosis in asymptomatic patients. Postoperative stroke or death rates of $6.3 \%$ and $4.4 \%$ were found in those with symptomatic and asymptomatic stenoses, respectively.

Conclusions: Improvement in the interpretation of carotid angiography is necessary to reduce the incidence of inappropriate surgery. The high rate of CEA for asymptomatic stenosis, as currently practiced in our region, is unjustified.

\section{F. CLINICAL NEUROLOGY AND EXPERIMENTAL THERAPEUTICS}

\section{F-01}

\section{AIDS Dementia Complex with Generalized Myoclonus}

J. Maher, S. Choudri, W. Halliday, C. Power, A. Nath (Winnipeg,Manitoba)

Background: Focal, segmental, and multifocal myoclonus have been described in patients with human immunodeficiency virus (HIV) infection.

Case Reports: We describe three patients with rapidly progressive HIV encephalopathy and generalized, predominantly proximal, myoclonus. Myoclonus manifested during the terminal stages of the illness. It was elicited by sudden auditory stimuli in two cases. Myoclonus was associated with an absent or inconsistent electroencephalographic event. Cortical somatosensory evoked potentials done in one patient were normal. Autopsy findings in one patient showed dramatic involvement of the brain stem, including the nucleus reticularis gigantocellularis bilaterally, though lesser changes were present throughout the brain; no opportunistic infection was present. 
Conclusions: HIV encephalopathy may be associated with generalized myoclonus and should be included as a cause of myoclonic dementia. We speculate that the myoclonus is subcortical in origin and that HIV is directly etiopathogenic in the clinical syndrome.

\section{F-02}

\section{A Case-Controlled Population-Based Study of Long-Term Glioblastoma Multiforme Survivors (LTGBMS) in Alberta From 1975-1991}

J.N. Scott, N.B. Rewcastle, P. Brasher, J. MacKinnon, N. Hagen, J.G. Cairncross, P. Forsyth (Calgary, Alberta), D. Fulton (Edmonton, Alberta)

Objectives: To determine 1) the frequency of LTGBMS in a population-based study, and 2) patient, tumor, and treatment factors which predict LTGBMS.

Methods: All Alberta residents with a histologic diagnosis of GBM between 1/1/75 - 12/31/91 were identified in the Alberta Cancer Registry and their charts reviewed. Histology was rereviewed by a blinded neuropathologist. LTGBMS were defined as those surviving $\geq 3$ years following diagnosis. LTGBMS were each compared to three age-, sex-, and year of diagnosis-matched controls and differences in patient, tumor, and treatment factors analyzed.

Results: 704 GBMs were diagnosed in the study period. Twenty (2.8\%) survived $\geq$ three years. In Southern Alberta, LTGBMS (average $=45$ years) were significantly younger when compared to all GBMs (average $=59$ years) $(\mathrm{p}=0.0001)$. The proportion of LTGBMS with a KPS $\geq 90$ at diagnosis was significantly greater $(p=0.001)$, compared to controls. However, of the various factors (including presenting symptoms, type and extent of both surgery and radiotherapy, tumor size and location, etc.) none was significantly different between LTGBMS and controls.

Conclusions: This study emphasizes the dismal prognosis for conventionally treated GBMs. LTGBMS are rare, more likely to be young, and have a high KPS. We could not identify other clinical predictive factors in Southern Alberta. LTGBMS had significant radiation-induced (RI) dementia but no RI neoplasms. We are investigating whether molecular studies can predict LTGBMS.

\section{F-03}

\section{Frontal Behavioural Inventory (FBI)}

\section{A. Kertesz, H. FoX, W. Davidson (London, Ontario)}

Frontal lobe dementia (FLD) is characterized by personality changes that are difficult to quantitate. In order to standardize and facilitate the diagnosis of FLD, a Frontal Lobe Inventory (FBI) of the symptoms was developed and studied in a population of FLD, early Alzheimer's disease (AD), and depressive pseudodementia (DPD). FBI is also studied in a non-selected population at entry point of our dementia clinic. Rèsults indicate a clear separation of FLD from AD and DPD. More advanced cases of AD develop some of the symptoms, but their scores do not approach the cut-off point for FLD. In conclusion, a quantifiable behaviour inventory is useful to operationalize the clinical criteria of FLD and serve as a diagnostic instrument at the entry point in a dementia clinic.

\section{F-04}

Use of Genotypic-Phenotypic Correlations Within CHIAARRA and COSTAR to Determine Liability to Develop Multiple Sclerosis and its Response to Drug Treatment: Preliminary Report

S.S. Beall, M.K. Hockertz, D. Studney, D.W. Paty (Vancouver, British Columbia)

Background: Research in MS involves many disciplines including genetics, immunology, neuroimaging and clinical neurology.

Interdisciplinary and multi centre collaborations require this information to be available in the compatible data formats necessary for intercommunication but also ensuring patient (MSCOSTAR) confidentiality.

Methods and Results: Longitudinal clinical (phenotypic) data on MS patients are recorded and stored in a computerised medical record (MS-COSTAR) which is based at the Vancouver MS Clinic. In another project, DNA derived from more than one thousand individuals comprising approximately 300 nuclear families of MS clinic patients has been collected. The DNA has been dissected and expanded into 21,000 markers defining the loci which encode proteins responsible for orchestration of the immune system. The DNA marker (genotypic) data are stored in a relational database management system (Oracle) which is the backbone of a computerized heuristic, CHIAARRA (Computerized Heuristic of Internet Accessible Alleles for Relational Retrieval and Analysis) used for automatic experimental design and analysis of the genetic data. A third database has information on serial MRI images of MS patients from a multi centre collaborative study. For collaborative research, we have been able to successfully link these three databases into one correlation matrix. Analysis of shifts of the genotypic-phenotypic correlations of placebo vs treatment arms of the recent beta-interferon trial (Paty et al, 1993) allows us to determine genotype-dependent drug responses.

Conclusions: By linking databases into the MS-COSTAROracle matrix, we have been able to establish correlation between genetic parameters, clinical characteristics, and disease measurements as well as drug treatment responses based on genotype-crucial information for collaborative research into the etiology, natural history and treatment of MS.

\section{F-05}

Enhanced Ex Vivo High Molecular Weight Neurofilament Subunit (NFH) Binding to Tubulin Following In Vivo Aluminum Chloride Exposure. Implications for the Pathogenesis of Amyotrophic Lateral Sclerosis

\section{K. Cunningham, M.J. Strong (London, Ontario)}

Background: To understand the pathogenesis of the intraneuronal aggregates of phosphorylated neurofilament (NF) that are an early feature of amyotrophic lateral sclerosis (ALS), we have utilized intracisternal inoculums of aluminum chloride $\left(\mathrm{AlCl}_{3}\right)$ in New Zealand white rabbits to replicate their formation. In this study, we have assessed the integrity of NFH/tubulin binding in this model. 
Methods: Four young adult New Zealand white rabbits were killed 48 hours following an intracisternal inoculum of $1000 \mu \mathrm{g}$ $\mathrm{AlCl}_{3}$. Highly phosphorylated NFH was isolated by electroelution of the $200 \mathrm{kDa}$ isoform from a NF-enriched cytoskeletal pellet. Tubulin was purified from pooled adult rabbit brains by repeated temperature-dependent polymerization and depolymerizations. NFH was dephosphorylated, incubated with tubulin in the presence of $20 \mu \mathrm{M}$ Taxol, $1.0 \mathrm{mM} \mathrm{MgCl} 2$ and $1.0 \mathrm{mM} \mathrm{GTP}$ for 30 minutes at $32^{\circ} \mathrm{C}$ and then centrifuged at $7,000 \mathrm{x}$ g for 10 minutes. The insoluble pellet, containing assembled NFH, and supernatent were separated and electrophoresed.

Results: Following dephosphorylation, $\mathrm{NFH}$ from $\mathrm{NaCl}$ inoculated rabbits was equally partitioned between the soluble and insoluble fractions. In contrast, following dephosphorylation, $\mathrm{NFH}$ isolated from Al-exposed rabbits completely bound to tubulin and partitioned exclusively to the insoluble fraction.

Conclusions: NFH dephosphorylation is the primary determinant of NFH/tubulin binding in vivo (Hisanaga et al., 1993). We have previously shown that a post translational modification $\mathrm{NFH}$ following $\mathrm{AlCl}_{3}$ exposure is the primary determinant of $\mathrm{NF}$ aggregate formation. These studies demonstrate that this process is accompanied by enhanced NFH binding, a process which occurs in vivo in the proximal axonal process at the site of NF aggregate formation in both aluminum neurotoxicity and ALS.

MJS is supported by a MRC Scholarship and by the ALS Society of Canada

\section{F-06}

Effect of Oral 4-Aminopyridine in the Reduction of Spasticity in Persons with Spinal Cord Injuries

P.J. Potter, K.C. Hayes, J.T.C. Hsien, G.A. Delaney, D.L. Wolfe (London, Ontario), D. GARDNER (Toronto, Ontario)

Preclinical trials of intravenous (24mg over 6 hours) and immediate release (IR) capsules $(10 \mathrm{mg})$ of 4-aminopyridine (4AP) in patients with spinal cord injury have previously yielded evidence of temporary reductions in spasticity. Three patients who responded well in the preclinical trials were administered 4-AP IR over a 3 month interval to monitor the potential therapeutic benefit. Two (1 Frankel C tetraplegic and 1 Frankel D tetraplegic) of the three patients received optimal benefit with a dosage of $10 \mathrm{mg}, \mathrm{PO}$, TID while the third (Frankel D tetraplegic) responded well after $10 \mathrm{mg}$, PO, BID. All three patients have experienced a decrease in tone and frequency of spasms. Quantitative assessment of ankle joint dynamics in response to passive sinusoidal displacement of the foot $(0.5,1.0,1.5$ and 2 $\mathrm{Hz}$ ) confirmed the subjective reports of reduced hypertonus. Probable unmasking of motor function has been demonstrated with improvement in functional abilities that may be secondary to the reduction in spasticity. Improved functional benefits include enhanced ability to stand supported, improved gait and stair climbing ability and increased ability to transfer as a result of the limitation of involuntary spasms. Other benefits experienced by these patients include improved bowel, bladder and sexual function, reduction of pain and perceived increase in energy reserves. Patients experienced no untoward side effects and will continue to be monitored for toxicity and tolerance. (Funded by Grant from The Parkwood Hospital Foundation)

\section{F-07}

Prolonged Suppression of CNS Anti MBP by Repeated IV Administration of MBP Synthetic Peptides Containing the Epitope Pro85 to Pro96 to Patients with Chronic Progressive MS

\section{K. WaRREn, I. CaTz (Edmonton, Alberta)}

Background: Subsequent to identifying MBP containing immune complexes in serum of patients with MS, free $(F)$ and bound (B) anti-MBP were purified by affinity chromatography from CSF and brain tissue of MS patients. Purified anti-MBP was utilized to localize the MBP epitope by synthetic peptide(s) analysis. The MBP epitope for MS anti-MBP was shown to be: $\mathrm{Pro}_{85}$-Val-Val-His-Phe-Phe-Lys-Asn-lle-Val-Thr-Pro ${ }_{96}$

Methods: Subsequent to obtaining University of Alberta Ethics Committee approval, selected synthetic MBP peptides containing the epitope between $\mathrm{Pro}_{85}$ and $\mathrm{Pro}_{96}$ were administered intrathecally and intravenously to MS patients with chronic progressive disease. CSF anti-MBP levels were monitored by a solid phase radioimmunoassay daily, weekly or monthly in order to determine the effect of the synthetic peptide(s) on CNS anti-MBP titers.

Results: Intrathecal administration of the immunodominant synthetic peptide suppressed CSF F anti-MBP titers for periods in excess of 7 days. Intravenous administration of the same peptide suppressed both $F$ and B CSF anti-MBP for periods up to 4 months and by 6 months CSF anti-MBP levels had returned to pretreatment values. A second intravenous injection of this peptide suppressed again both F and B CSF anti-MBP for periods of 6-12 months.

Conclusion: Synthetic peptides containing the linear $\mathrm{L}$ amino acid sequence of human MBP $\left(\mathrm{Pro}_{85}-\mathrm{PrO}_{96}\right)$ can be administered safely intrathecally or intravenously to MS patients with chronic progressive disease resulting in suppression of CNS anti-MBP titers. The therapeutic value of this observation has yet to be determined by double blind Phase II and III clinical research.

\section{F-08}

\section{Therapeutic Potential of a Neurotrophin Antagonist}

R.J. Riopelle, G.M. Ross, D.F. Weaver, M.A. Bisby, (Kingston, Ontario), M. FaHNestock, R.J. RaCINE (Hamilton, Ontario)

Background: Sustained changes in functional properties of primary sensory neurons likely underlie the pain associated with chronic inflammation (arthritis, interstitial cystitis, inflammatory bowel disease), reflex sympathetic dystrophy associated with soft tissue injury, and nerve insult (injury, neuropathy). In models of chronic pain both peripheral and central aberrant sprouting of neuronal processes is observed. In the central nervous system, development of the epileptic state is associated with 
morphological evidence of aberrant neuronal process sprouting in humans and in experimental epilepsy models. Neurotrophins have been implicated in both chronic pain and epilepsy.

Methods: Emerging from our program to develop small molecule antagonists of neurotrophin function in vitro (see companion abstract Riopelle, Ross, Shamovsky, Weaver), we have begun to utilize well-characterized in vivo models replicating features of the disorders of interest to seek proof of concept that neurotrophins are involved in pathogenesis.

Results and Conclusions: The therapeutic activity of one such antagonist is associated, in part, with inhibition of aberrant neuronal process growth.

Supported by The Neuroscience Network, NSERC (Canada), MRC (Canada), NIH (USA), and Allelix Biopharmaceuticals (Mississauga, Canada).

\section{F-09}

\section{Restoration of the Nigrostriatal Circuitry by Fetal Nigral Grafts in the Striatum and Nigra: Evidence of Functional and Anatomical Recovery}

\section{Mendez And M. Hong (Halifax, Nova Scotia)}

Background: It is well known that grafts of fetal nigral tissue survive and grow in the dopamine (DA) depleted adult striatum. Although such grafts produce reversal of behavioural and biochemical deficits in the rat model of Parkinson's disease (PD), little is known about the restoration of the nigrostriatal circuitry following transplantation.

Methods: To further study the functional and anatomical recovery produced by neural transplantation, we have employed a novel micrografting technique to stereotaxically implant fetal nigral dopaminergic cell suspensions into both the striatum and substantia nigra of female, Wistar rats (200-225 g) bearing unilateral 6-hydroxydopamine (6-OHDA) lesions of the nigrostriatal dopaminergic pathway. Analysis of functional recovery was carried out using a video activity monitor to assess animal locomotor activity and analysis of anatomical recovery was made using tyrosine hydroxylase ( $\mathrm{TH}$ ) immunohistochemistry.

Results: A pre-graft challenge with amphetamine $(5 \mathrm{mg} / \mathrm{kg}$ i.p.) induced asymmetric turning behaviour towards the lesioned side: $566.2 \pm 84.5$ ipsiversive rotations vs $0.7 \pm 0.4$ contraversive rotations over a 60 minute observation period (mean \pm $\mathrm{SEM}, \mathrm{n}=6$ in each group). In contrast, a post-graft challenge at 6 weeks with amphetamine failed to induce asymmetric turning behaviour. Immunohistochemical analysis using an antibody against $\mathrm{TH}$ revealed a large number of $\mathrm{TH}$-positive cell bodies in the grafts both in the striatum and substantia nigra. A dense network of dopaminergic processes was also found within the grafts and a large number of axons projected from the nigral graft toward the striatal graft and viscera. This produced restoration of the nigrostriatal pathway.

Conclusions: This study for the first time demonstrates both functional and anatomical restoration of the nigrostriatal pathway by concurrent fetal nigral grafts in the striatum and nigra in the 6-OHDA rat model of PD.

\section{F-10}

\section{Pallidotomy in Parkinson's Disease: Short Term Results}

A. Kishore, I. Turnbull, R. De la Fuente-Fernandez, C. Honey, D.B. CALne, B.J.SNow (Vancouver, British Columbia)

Objective: To determine the effects of unilateral pallidotomy in Parkinson's Disease (PD).

Background: Recent studies suggest that ventrolateral pallidotomy improves parkinsonian signs and dyskinesias.

Methods: Eighteen patients $(\mathrm{m}=11, \mathrm{w}=7$, mean age $61.6 \pm 11)$ who underwent stereotactic unilateral ventrolateral pallidotomy (VPLP) were rated within two weeks before and up to three months after surgery using UPDRS (subsets: Motor ADL and motor complications). The severity of dyskinesias was rated by the scale of Goetz CG et al. Patients were rated both OFF (12 hours off medication), and ON (optimally medicated with levodopa/carbidopa). The results were analyzed with regression methods, adjusting for age, duration of follow up and scores pre-operatively when relevant.

Results: Motor UPDRS score in the OFF state improved in all ( $\mathrm{p}<0.0001$ ):64\% contralaterally, and $26 \%$ ipsilaterally. All the major parkinsonian signs improved (tremor $76 \%$, rigidity $53 \%$, bradykinesia, $39 \%$ and postural stability $39 \%$ ). Total dyskinesia score improved in all patients $(p=0.001)$. Dyskinesias improved contralaterally by $78 \%$, and ipsilaterally by $34 \%$. ADL in the OFF state improved by $40 \%(\mathrm{p}=0.005)$. In the ON state there was improvement in both UPDRS motor scores $(p=0.001)$ and ADL $(p=0.03)$. Daily levodopa dose did not change after surgery.

Conclusions: Unilateral VPLP in PD improves substantially the motor deficits and dyskinesias contralaterally. Follow-up up to 3 months showed ipsilateral improvement to a lesser degree.

\section{F-11}

\section{Evaluation of Electroconvulsive Therapy in Parkinsonism}

Y. Wang, J.K.C. Tsui, A.M. Jiwa, D.B. Calne (Vancouver,British Columbia)

Twenty patients with idiopathic Parkinson's disease (IP) received electroconvulsive therapy (ECT) from 1980-1995. Each course of ECT included 3-5 sessions, and six patients had 2 or 3 courses ECT. There was substantial improvement in 12 patients. Benefit was obtained in delusional psychosis, suicide ideation, and visual or auditory hallucinations. In addition, there was major amelioration of Parkinsionian deficits in 13 patients. Rigidity, and bradykinesia were improved, while, dyskinesia and dystonia decreased in 3 patients. The duration of ECT effects ranged from 3 weeks through 6 months when the last follow-up observation was made. Side effects included producing transient deterioration of short term memory in 11 patients, exacerbating agitation and paranoia in 3 patients.

Our study indicates that ECT has a useful role in complicated IP, but it is contraindicated in patients with significant intellectual impairment. Our findings, together with previous reports, suggest that ECT is under utilized in difficult management situation with IP. 


\section{G. EPILEPSY}

G-01

\section{Expert System Approach For Analyzing Electrographic Seizures}

\section{S.S. Seshia, L. Yao, E. Shwedyk (Winnipeg, Manitoba)}

Computer aided analyses facilitate seizure detection. Current approaches are based on conventional signal processing methods. To the best of our knowledge, expert system principles have not been tried. We describe a prospective study using such a technique.

The rules for recognizing electrographic seizures were predefined. The rules were programmed on a MacIntosh computer using $C$ language and CLIPS. Independent training $(\mathrm{N}=40)$ and evaluation $(\mathrm{N}=85)$ sets of $E E G$ data (20 seconds each) incorporating electrographic seizures identified by a clinical neurophysiologist were selected from records done with a 16 channel ambulatory recorder, bipolar montages being used. The EEGs were done for clinical reasons, primarily to obtain electrographic correlates to clinical seizures. The performance of the computer based expert system was compared with that of the clinical neurophysiologist. 142/424 "events" identified by "the system" as real or suspect electrographic seizures were not labelled as such by the neurophysiologist (False + rate of $34 \%$ ). Conversely, "the system" missed 19/301 "events" (false - ve rate 6\%) identified as real or suspect by the neurophysiologist. We suggest that expert system methods may have a useful role in helping us detect electrographic seizures in prolonged recordings.

Funded by: Health \& Welfare Canada/HSCRF

\section{G-02}

Long-Term Open-Label Study of Topiramate: The Canadian Experience

A. Guberman, M. Couture (Ottawa Ontario), J. Bruni, J. SCHNEIDERMAN (Toronto,Ontario)

Background: Topiramate (TPM) is a novel antiepileptic drug active in the rodent MES epilepsy model and has been shown to be safe and effective in a variety of seizure types in humans.

Methods: Two Canadian centres have participated since 1993 in a multicentre, open, add-on study of TPM in adult epilepsy. Adults over 18 years old with a definite diagnosis of epilepsy (any seizure type) who were felt to be poorly controlled were included. Topiramate was initiated at $100 \mathrm{mg} /$ day (later 50 $\mathrm{mg} / \mathrm{d}$ ) and titrated upwards to maintenance dose (maximum $1000 \mathrm{mg} /$ day) as tolerated. Safety parameters as well as seizure response were assessed.

Results: Twenty-nine of the 36 patients had partial seizures with or without secondary generalization. The mean maximum dose of TPM was $441.7 \pm 275.8 \mathrm{mg} /$ day. Overall median percent seizure reduction was $46.9 \%$ although 5 patients remained $<30$ days and $7<60$ days in the study. In all 26 patients were discontinued: 7 for ineffectiveness, 15 for adverse drug effects, 3 for protocol violation and $\mathrm{I}$ at the patient's request. Ten patients remain on the drug for a mean of 24.4 months with $4 / 11$ showing $>90 \%$ seizure reduction. There were no serious adverse events. The most common treatment emergent side effects were fatigue $(38.9 \%)$, memory difficulties $(36.1 \%)$, headache (30.6\%), weight loss $(33.3 \%)$, cognitive slowing (22.2\%), dizziness $(22.2 \%)$.

Conclusion: Topiramate is an effective, easy to use, and safe add-on agent for long-term treatment of uncontrolled adult epilepsy. The relatively high dropout rate in our study may be partially due to the high initial dose $(100 \mathrm{mg})$.

\section{G-03}

\section{Survey of Canadian Neurologists Regarding Epilepsy and Driving}

\section{R.S. MCLACHLAN, (London, Ontario)}

Certain neurological disorders increase the risk of motor vehicle accidents, but there is controversy as to who should determine medical eligibility to drive, the treating physician or the state.

Method: A questionnaire sent to all adult neurologists in Canada ( $\mathrm{N}=494)$ asked about current practice and opinions regarding neurological disorders and driving. Responses were compared between neurologists practicing in provinces with and without legislation requiring compulsory reporting of medical conditions.

Results: There were $289(59 \%)$ responses with the lowest provincial response rate $46 \%$. Overall, $50 \%$ of neurologists reported seizures most of the time compared to $4 \%$ for stroke/TIA, $26 \%$ for dementia and $8 \%$ for other neurological conditions $(p<.0001)$. In five provinces with mandatory reporting legislation, $84 \%$ of neurologists $(\mathrm{N}=140)$ reported seizure patients most of the time, compared to $19 \%(\mathrm{~N}=149)$ in other provinces $(p<.0001)$. No differences appeared in reporting of other conditions. Agreement with mandatory reporting was $44 \%$ but this differed in mandatory vs. discretionary reporting provinces (63\% vs. $26 \%$, p<.0001). Only $11 \%$ thought medical guidelines regarding driving were inadequate but $51 \%$ indicated the minimum seizure free interval should be six months or less.

Conclusion: Seizures are selectively reported more often than other neurological conditions but there is considerable variation in opinion and practice of neurologists depending on province of residence.

\section{G-04}

Quebec City Epilepsy Surgery Program : The First Three Years

S. Tremblay, R. Desbiens, C. Picard, D. Lacerte, M. Thibault, PV. Gould, S. VerRet (Quebec, Quebec)

Background: Epilepsy surgery has been performed in Quebec City since 1992, using standard investigative methods but no invasive intracranial evaluations. We present our results and 
compare them with the surgical series of established epilepsy surgery programs.

Methods: Updated medical records were reviewed. Engel's classification was used for assessment of seizure control in surgical resections. Patients were divided in 3 groups: group 1: temporal lobe surgeries; group 2: extratemporal lobe surgeries; group 3: callosotomies.

Results: Forty-one surgeries were performed, 26 in group 1, 5 in group 2 and 10 in group 3 . Twenty-one $(80 \%)$ in group 1 and 2 (40 $\%)$ of group 2 are Engel class 1 . Nine $(90 \%)$ patients in group 3 are significantly improved $(>50 \%$ reduction in drop attacks and tonic or tonic-clonic seizures). Followups are between 0-40 months.

Conclusions: Our results are comparable with published surgical series from established epilepsy surgery programs, but should be interpreted cautiously until extended follow-up becomes available. Epilepsy surgery can be performed efficiently with excellent results in smaller epilepsy surgery programs with careful case selection. This approach hastens access to surgery and allows ultraspecialized centers to concentrate on difficult cases requiring invasive studies.

\section{G-05}

\section{More Severe Hippocampal Damage When Medial Temporal Lobe Epilepsy Is On The Dominant Side For Language}

A. Bouthillier (Montreal, Quebec), D.D. SPEnCER, J.H. Kim, M. Luby, G. McCarthy, K.J. Sass (New Haven, Connecticut)

Rationale: Long-term synaptic potentiation has been implicated in hippocampal memory processing and could be involved in epileptogenicity. Since memory appears to be temporal lobe side specific and better defined on the dominant side for language, this study was undertaken to evaluate hippocampal damage as a function of the dominant or non dominant side of medial temporal lobe epilepsy (MTLE).

Methods: Hippocampal subfield neuronal densities and hippocampal volumes (HV) as determined by volumetric MRI were prospectively collected in 90 consecutive patients undergoing surgery for non lesional MTLE. All patients had language dominance established by the Wada test.

Results: In patients with a history of febrile seizure (FS) $(n=44)$, all subfields except $\mathrm{CA}^{2}$ showed more neuronal loss when the disease was on the dominant side as compared to the non dominant: $\mathrm{CA}^{\prime}, 81 \%$ vs. $68 \%(\mathrm{p}=0.0002) ; \mathrm{CA}^{3}, 71 \%$ vs. $55 \%$ $(\mathrm{p}=0.002) ; \mathrm{CA}^{4}, 78 \%$ vs. $57 \%(\mathrm{p}=0.002)$; granular cell layer, $60 \%$ vs. $47 \%(p=0.007)$. In patients with no history of FS $(n=46)$, those differences were not significant. HV on the side of disease was not influenced by the dominance, but contralateral HV were smaller when on the dominant side ( 1.28 vs. $1.47 \mathrm{~cm}^{3}, \mathrm{p}=0.05$ ).

Conclusion: MTLE is associated with more hippocampal damage when the disease is on the dominant side for language, but only if the etiologic risk factor is a FS. This finding could be related to more potent excitotoxicity in the dominant hippocampus, or to undetermined disease specificity associated with FS.

\section{G-06}

Postoperative Outcome of Seizures and Aura Correlated with the Extent of Mesial Temporal Resection in Temporal Lobe Epilepsy (TLE)

R. Morris, M.A. Alonso-Vanegas, V.K. Srivastava, U. Lee, L.F. Quesney, A. OliviER. (Montreal, Quebec)

Background: Many patients with TLE can have complete postoperative relief of seizures and aura. Yet, some patients have persistent auras.

Methods: Twenty seven cases of non lesional TLE patients with seizure and aura operated at the Montreal Neurological Institute (MNI) from 1976 - 1994 were analysed. There were 16 males and 11 females. The age distribution ranged from 15 to 54 years. EEG changes were unilateral in 21 , in 6 the abnormalities were bilateral but with clear unilateral predominance. The mean follow up period was 6.87 years.

Results: 14 patients $(52 \%)$ had relief of both seizure and aura, $9(33 \%)$ were seizure free but aura persisted, 3 had a diminished frequency and intensity of seizures and aura, 1 had less disabling seizures with no aura. Six patients underwent radical removal of the amygdala and $3.5 \mathrm{~cm}$ of the hippocampus. Five patients $(83 \%)$ were free of seizures and aura; in one, the aura was still present but remained seizure free.

Conclusions: Radical removal of the amygdala and $3.5 \mathrm{~cm}$ of the hippocampus gave optimal relief of both seizures and aura. The anatomical substrate for the origin of seizures and aura in non lesional temporal lobe epilepsy patients are different.

\section{G-07}

Role of the Amygdala and Entorhinal Cortex in the Surgery of Temporal Lobe Seizures

W. Feindel, G. Leonard, Z. Caramanos, M. Ll, J. Harrison, A. KuCHEla (Montreal, Quebec)

Background: Various structures of the mesial temporal region, the amygdalo-hippocampal complex, the cortex of the rhinal, insular and entorhinal areas and the subiculum, have been implicated in the pathogenesis of temporal lobe epilepsy. Their relative significance, however, taken individually or together, in relation to the anatomical patterns of surgical excision that result in successful outcome, still remains controversial.

Methods: In 50 consecutive patients with temporal lobe seizures selected for surgical treatment from March 1985 to August 1988, using similar preoperative criteria, resection by the same surgeon involved temporal cortisectomy, radical amygdalectomy and minimal hippocampal removal. All patients had post-operative magnetic resonance imaging (MRI) to define the extent of the surgical excision. Response to a follow-up questionnaire was received from 46 of the 50 patients at a minimum of two years after surgery.

Results: The outcome in relation to seizure control was considered satisfactory in $90 \%$. Of these, $76 \%$ were seizure-free or became seizure-free within two years of the operation. An additional $15 \%$ reported a marked reduction in seizure tendency. In $9 \%$, there was less significant or no improvement in seizure control.

Conclusion: By comparison of various mesial temporal lobe 
excisions as described from other neurosurgical centres with the present results, we conclude: 1) The amygdala and entorhinal cortex represent the common critical region for a resection that achieves successful seizure control. 2) Major excision of the hippocampus is not essential for seizure control in many patients with temporal lobe epilepsy.

\section{G-08}

Treatment of Mesial Temporal Lobe Seizures by Stereotactic Ablation of the Amygdala and Hippocampus

\section{A.G. Parrent, W.T. Blume (London, Ontario)}

Treatment of intractable temporal lobe seizures with temporal lobectomy results in substantial seizure reduction in over $90 \%$ of patients. More recently selective amygdalohippocampectomy has been reported to alleviate seizures in appropriately selected patients (Weiser, 1991). In the past stereotactic amygdalotomy had been used to treat intractable seizures associated with behavior disorders, with some success (Narabayashi et al, 1963). This study was designed to assess the technical feasibility and preliminary results of stereotactic radiofrequency ablation of mesial temporal structures for the treatment of temporal lobe seizures.

A trial of stereotactic ablation of the amygdala and hippocampus was carried out in 10 patients with complex partial seizures of mesial temporal origin. Seizures originated from the left side in 7 patients and right side in 3. Mean age was $30 \mathrm{yrs}$ (17-42). All patients demonstrated a predominant unilateral temporal lobe spike/seizure focus. MRI demonstrated hippocampal high signal $+/$ - atrophy, compatible with mesial temporal sclerosis.

Using the Leksell stereotactic frame, volumetric MRI imaging was performed and reconstructed as thin contiguous axial and coronal images to allow the selection of multiple target sites in the hippocampus and amygdala. A multicontact electrode was stereotactically placed in the temporal horn of the lateral ventricle to record from the surface of the hippocampus during the procedure and for 48 hours post-operatively. The lesioning electrode (1 $55 \mathrm{~mm}$ tip) was advanced to each target through a single lateral temporal burr hole, and radiofrequency lesions were made $\left(75^{\circ} \mathrm{C}\right.$ $x 90 \mathrm{sec}$ ). It was not uncommon for isolated seizures to occur in the first 4-6 weeks after surgery (4 patients). Over a follow-up period of 1.16 months, 3 patients were seizure free, 5 experienced $>90 \%$ seizure reduction, 1 a $50-90 \%$ reduction and 1 $<50 \%$ reduction. There have been no complications.

Our experience suggests that stereotactic amygdalohippocampotomy may provide an effective and less invasive alternative to temporal lobectomy or selective amygdalohippocampectomy.

\section{G-09}

Evidence for Progressive Hippocampal Damage in Patients with Intractable Temporal Lobe Epilepsy and History of Febrile Seizures

A. Bouthillier (Montreal, Quebec), D.D. SPENCER, D. King, S.S. Spencer, M. Luby, G. McCarthy, J.H. Kim, K. J. Sass (New Haven, Connecticut)
Rationale: Medial temporal lobe epilepsy (MTLE) is often associated with structural and functional hippocampal damage. We investigated the hypothesis that the injury is progressive and looked for associated risk factors.

Methods: Hippocampal volumes (HV), memory scores (Selective Reminding Test), IQ scores and hippocampal neuronal densities were prospectively obtained in a series of 90 consecutive patients undergoing surgery for non lesional MTLE.

Results: There was a significant negative linear correlation between duration of epilepsy and HV on the side of surgery, but only in the group of patients with a history of FS ( $r=-0.571$, $\mathrm{p}=0.001)$. HV contralateral to the side of surgery was also influenced by the duration of epilepsy $(r=-0.469, p=0.009)$. For patients with FS and disease on the dominant side for language, there was a significant negative correlation between duration of epilepsy and verbal memory score $(r=-0.692, p=0.007)$, non verbal memory score $(r=-0.633, p=0.02)$, VIQ $(r=-0.496, p=0.02)$, PIQ ( $r=-0.435, p=0.04)$ and FSIQ $(r=-0.518, p=0.02)$. Mean hippocampal neuronal density also correlated with duration of epilepsy.

Conclusion: MTLE progresses differently dependent on a history of FS. Progressive bilateral, structural and functional hippocampal damage occurs only in the FS group.

\section{G-10}

\section{Use of Invasive Monitoring to Lateralize Seizure Foci in} Children

O.C. SNEAD III (Toronto, Ontario), S.R. Kongelbeck (Los Angeles, California), C. RAFFEL (Rochester, Minnesota)

Background: The epileptogenic zone in children undergoing evaluation for epilepsy surgery often may be extratemporal and require ictal recording from chronically implanted subdural electrode grids for localization prior to surgery. However, there may be insufficient data in some children to allow for lateralization of the ictal focus. Epilepsy surgery may be precluded for this group of children since lateralization of the epileptogenic zone is required in order to place a subdural grid for localization prior to surgery.

Methods: Seizures were recorded from bilateral subdural strip electrodes over a period of 3-8 days in 8 children who demonstrated clinical evidence of partial seizures, but had discordant EEG, neuroimaging, and clinical data which made lateralization of the epileptogenic focus impossible.

Results: Lateralization of the seizure focus was accomplished in seven of the eight children. All seven had subdural electrode grids placed subsequently over the side lateralized by subdural strip recordings with localization and focal cortical resection of the epileptogenic zone. All of the children with focal cortical resection had abolition of seizures or a significant reduction in seizure frequency.

Conclusion: This technique makes surgery an option for those children with intractable seizures who would otherwise be excluded from surgical consideration because of an inability to lateralize the seizure focus by noninvasive means. 


\section{G-11}

Linkage Study of Autosomal Dominant Febrile Convulsions in Two Multigenerational Pedigrees

\author{
L.J. Racacho, J. Maher, G.C. Ebers, R.S. McLachlan, D.E. \\ BULMAN (London, Ontario)
}

Febrile convulsions (FC) are clonic or tonic-clonic seizures in the presence of fever without any signs of intracranial infection, antecedent epilepsy, or other definable cause. They occur in 1 to $5 \%$ of all children between the ages of 1 to 6 years, making it the most common neurological disorder among children. Since twin and family studies support a genetic basis for $\mathrm{FC}$, we performed linkage analyses on two multigenerational families which appear to be segregating the disorder as an autosomal dominant trait with reduced penetrance. We investigated the possibility that any of five previously mapped epilepsy loci could be involved with seizure susceptiblity in FC. Microsatellite markers tightly linked to these epilepsy loci were tested for linkage to the FC trait at reduced penetrances of $70 \%$ and $95 \%$. Combined lod scores of less than -2.00 in both families indicated a 100:1 odds against linkage of any of these epilepsy loci to FC. Thus, our preliminary linkage analyses of the two families have excluded five previously mapped epilepsy loci, juvenile myoclonic epilepsy (EJM1), benign familial neonatal convulsions (EBNI and EBN2), Unverricht-Lundborg disease (EPM1), and a partial epilepsy locus. Furthermore, we could not replicate the linkage of FC to chromosome 8q13-21 suggested by a recent study.

\section{H. GENERAL NEUROSURGERY}

\section{H-01}

\section{Myelin - Associated Glycoprotein Influences Axon/Neutrite Growth and Growth Cone Motility}

\section{K. G. McKenzie Memorial Prize for Basic Neurosciences Research - 2nd prize}

M. Li, A. Shibata, C. Li, P.E. Braun, L. McKerracher, J. RoDer, S.B. KaTER, S. DAVID (Montreal, Quebec)

We have previously shown that myelin-associated glycoprotein (MAG) contributes to the neurite growth inhibitory activity in bovine central nervous system (CNS) myelin. Here we report studies on MAG-deficient mice, and the effects of recombinant MAG on neurite growth and growth cone collapse. Although there was no significant difference in the amount of neurite growth on myelin purified from MAG-/- and MAG+/+ mice, octylglucoside extracts of CNS myelin fractionated by DEAE-chromatography showed that the major peak of inhibitory activity that is associated with MAG in normal mice, is significantly reduced in MAG deficient mice. Axon regeneration was also examined in vivo after thoracic lesions of the corticospinal tracts. In contrast to $M A G+/+$ mice, a small number of anterogradely labeled axons extended up to 13.2 $\mathrm{mm}$ past the lesion in MAGdeficient mice. Additional in vitro studies demonstrated that recombinant MAG inhibited neurite growth and caused collapse of axonal growth cones. These data suggest that other myelin-derived inhibitors may compensate for the absence of MAG in the MAG-/- mice, and that MAG inhibits neurite growth by altering that behavior of axonal growth cones.

\section{H-02}

\section{Block Funding -The Manitoba Managed Care Plan}

\section{N.C. HILL (Winnipeg, Manitoba)}

Neurosurgeons in Manitoba have been convinced by Government and University officials to participate in a proposal in which it is claimed that costs will be reduced, dependence of the University on the financial support by practising physicians will be eased, and, by provision of adequate facilities and guaranteed income, the chronic shortage of neurosurgical manpower in Manitoba will be overcome.

Seven neurosurgeons, or their equivalent, will be funded to supply all of the neurosurgical services, both clinical and academic, in Manitoba and the Government through its Health Insurance Program will supply a block of money to neurosurgeons for this purpose. Negotiations included a guarantee of the preservation of the independent economic status of each neurosurgeon; the expenses to be included, (e.g. office costs) or excluded (e.g. malpractice insurance); the precise nature of services to be provided; quality assurance; and other considerations. The contract is for three years and will be described in detail.

The institution of such a plan (which is also being proposed for neurologists), if adopted on a national scale will have significant repercussions on the pattern of neurosurgery and neurology practice in Canada and on the teaching programs supplying neurosurgeons and neurologists to that practice.

\section{H-03}

The Health Utilities Index: A Useful Tool for Outcome Measurement and Quality Assurance in Neurosurgical Practice

\section{J. WeLls (Hamilton, Ontario)}

Background: The McMaster Health Utilities Index (HUI) provides self-reported measurement of health status in the following domains: vision, hearing, speech, mobility, dexterity, mood, memory, problem solving, self care, and pain. A simple questionnaire produces information suitable for interval level statistical testing. It has been fully validated. We investigated the HUI as an outcome measure and quality assurance tool for neurosurgical cases.

Methods: Neurosurgeons and rehabilitation specialists administered the HUI to hundreds of patients in their offices and clinics for three months. Acceptance, problems, and results were monitored and analyzed. A computer database and a format for analysis and presentation of the HUI test results were developed.

Results: HUI testing was easily done. Data highly suitable for outcome measurement and quality assurance were obtained. The test was not useful for people with severe dysphasia or for those speaking neither English nor French Canadian.

Conclusions: The HUI provides data useful for outcome 
measurement and quality assurance measurements for a wide variety of neurosurgical cases. It is helpful for management of individual cases, assessment of a neurosurgical practice, and for analysis of various neurosurgical patient populations. It provides data that are relevant, easy to understand, and suitable for interval level statistical analyses.

\section{H-04}

Analysis of 1594 Cases of Catastrophic Injuries in Sports and Recreation with a View to Injury Prevention

\section{C.H. TATOR, V. EDMONDS, L. LAPCZAK (Toronto, Ontario)}

The purpose of this study was to determine the epidemiology of catastrophic injuries in sports and recreation in Ontario in order to develop targeted prevention programs. From 1986 to 1992, three surveys were conducted each of 12 months duration. The surveys were designed to identify all catastrophic injuries occurring due to sports or recreational activities in the Province of Ontario in each 12 month period. Catastrophic injury was defined as "an injury causing actual or potential long-term and/or permanent injury, or an injury causing death".

A total of 1594 catastrophic injuries were identified consisting of 1071 survivors and 523 fatalities. The main types of injuries were to the head and spine. Eighty-three \% were male and $17 \%$ were female. For both male and females, the peak decade for injury was the 11-20 year old group which accounted for $31.2 \%$ and $30.6 \%$ of the injuries in females and males, respectively. The mean and median ages for the fatalities were 31.3 and 29.0 years, respectively. Water sports were the leading cause of injury accounting for $27.3 \%$ of all injuries with motor sports second at $19.3 \%$, and winter sports third at $15.7 \%$. Bicycle injuries were the fourth most common cause at $10.3 \%$. Water sports accounted for $55.5 \%$ of the 523 fatalities, while $22.6 \%$ were due to motor sports, and $8.6 \%$ were due to bicycling. In all activities except equestrian sports, males out numbered females.

Some important trends were noted during the course of this study including a marked rise in snowmobile injuries and a decline in hockey injuries.

The information obtained in these surveys has been the basis for the development of targeted prevention programs which are now underway in many sports and recreational activities including hockey, snowmobiling, diving and other water activities, and racket sports.

\section{H-05}

Is Lateral Plain X-ray of the Cervical Spine Adequate for Ruling Out Cervical Spine Fractures in Severe Head Injury Patients?

\section{E.A. BADEJo, R.R. SAlumbides (Kearney, Nebraska)}

Background: Patients with severe head injury are presumed to have a cervical spine injury until this is proven otherwise. To prove the absence of cervical spine fracture a lateral $x$-ray of the cervical spine was deemed adequate provided it showed the cer- vical spine up to $C 7$. In the last 2 years we have found increasing numbers of false negative plain $x$-rays of the cervical spine, and the essence of this paper is to present our findings in the last 2 years.

Methods: We reviewed all our patients admitted to the hospital with head injury who had cervical spine $x$-rays and also CT scan of the spine, and compared the plain $x$-ray findings with the CT scan findings for any discrepancies.

Results: Over the 2 year period there were 8 patients with negative cervical spine $x$-rays who had fractures of the cervical spine on CT. Two of these patients eventually required cervical fusion with plating. In one of the patients the CT scan was negative, but the MRI showed a fracture dislocation of the cervical spine.

Conclusion: Plain cervical spine $\mathrm{x}$-rays are not adequate to rule out cervical spine fractures. This is especially the case in unconscious patients who are unable to complain of neck pain. The routine in our hospital in such severe head injury patients is to do a CT scan of the cervical spine also.

\section{H-06}

Factors Predictive of Hospital Mortality and Mechanical Ventilation in Patients with Cervical Spinal Cord Injury

\author{
M. Fehlings, A. Claxton, D. Wong, F. Chung \\ (Toronto,Ontario)
}

Background: The objective of this study was to identify factors predictive of mortality and the need for mechanical ventilation in patients with cervical spinal cord injury $(\mathrm{SCl})$. To date a multivariate analysis of independent predictors of these clinical outcomes has not been performed.

Methods: A retrospective analysis of all patients admitted to the Toronto Hospital with major cervical SCI between 1982 and 1994. Univariate and multivariate logistic regression were used to identify factors predictive of mortality and ventilator-dependent respiratory failure.

Results: Data were analyzed on 72 cases. All patients were managed in a neurosurgical intensive care unit. 15 patients (21\%) died in the first 3 months post-injury and 41 patients $(57 \%)$ required mechanical ventilation. Univariate analysis identified age, pre-existing cardiac disease, neurological level at $\mathrm{C} 4$ or above, Glasgow Coma Scale (GCS) $<14$, vital capacity and an absent cough to be associated with mortality. Factors associated with the need for mechanical ventilation included neurological level at $\mathrm{C} 5$ or above, complete $\mathrm{SCl}$, copious sputum, major lobar collapse and pneumonia.

Conclusions: Multivariate logistic regression identified age, level of cord injury and GCS as independent predictors of mortality in patients with cervical SCl. Copious sputum and pneumonia were independent predictors of the need for prolonged mechanical ventilation. 


\section{H-07}

\section{Anterior Laparoscopic Lumbar Fusion(BAK System): Preliminary Experience}

\author{
S. Chakravarthi, A.G.E. North, R.R. Anderson, R. Lemmon \\ (Windsor, Ontario)
}

Background: Recently minimally invasive laparoscopic surgical techniques have been developed to perform lumbar discectomy and fusion at the L5-SI intercertebral disc space. Twelve patients are included in this study. Nine patients had a diagnosis of a symptomatic Grade I spondylolisthesis. One patient had a recurrent disc herniation following two previous posterior decompressions. Two additional patients had chronic back pain associated with a central disc herniation.

Method: The procedure was carried out through standard laparoscopic portals with a working channel positioned over the L5-SI disc space. The disc space is distracted, disc material removed and two metal cages packed with autologous bone graft are then screwed into the intervertebral space. The patients are mobilized immediately.

Results: There were no complications. In two patients there were technical limitations inserting two BAK cages and only one was used. In all cases the spondylolisthesis was reduced and maintained on serial lumbar $x$-rays. All of the BAK cages remain in position.

Conclusion: We are presently following all patients with a detailed assessment in order to critically evaluate the long-term outcome. The challenge in lumbar fusion procedures remains the indications and patient selection.

\section{H-08}

Time Course of Cerebral Ischaemia Following Traumatic Brain Injury

J. Brown, A.J. Baker, S.J. Konasiewicz, R.J. Moulton (Toronto, Ontario)

Background: Evidence of cerebral ischaemia is frequently found at autopsy after fatal traumatic brain injury (TBI) but clinical demonstration of cerebral ischaemia leading to neurological deterioration is difficult. Discriminate analysis defines a group of patients with elevated arteriovenous oxygen difference $\left(\mathrm{AVDO}_{2}\right)$ within 24 hours of injury and deteriorating somatosensory evoked potentials (SSEPs) over a five day period. This paper compares time courses of ischaemia and electrophysiological change in this group.

Methods: From July 1987 to November 1995, intensive monitoring of 46 patients with severe TBI (GCS $\leq 8$ after resuscitation) included twice daily $\mathrm{AVDO}_{2}$ and cerebral blood flow (CBF) measurements and hourly SSEPs for an average of five days. SSEPS were quantitated as summed peak to peak amplitudes. Five ischaemic patients were identified by discriminate analysis.

Results: Ischaemic patients did not differ in initial SSEPs from the nonischaemic group but deteriorated to SSEP amplitudes significantly below those of the nonischaemic group by 48 to 120 hours post injury. The mean CBF in ischaemic patients was initially significantly below that of the nonischaemic group
( 30.01 vs. $46.91 \mathrm{cc} / 100 \mathrm{~g} / \mathrm{min}$ respectively, $\mathrm{p}=0.001$ ) but by 24 hours post injury was not significantly lower than nonischaemic patients.

Conclusions: Electrophysiological deterioration persists beyond the period of ischaemia in TBI patients undergoing ischaemic secondary injury and is concomittant with recovery of CBF (reperfusion). A definition of ischaemic secondary injury based on both electrophysiological deterioration and elevated $\mathrm{AVDO}_{2}$ is useful because thresholds for metabolic failure remain undefined in this pathophysiologic setting and may differ from accepted values.

\section{H-09}

Serial Median Nerve Somatosensory Evoked Potentials Obtained Within the First Week After Injury as Predictors of Outcome in Comatose Patients with Head Injuries

D.A. Houlden, K.A. Klettke, R.P. Henriques, D.G. BaSavakumar, M. L. Schwartz (Toronto, Ontario)

Background: Early median nerve somatosensory evoked potential (SSEP) grade predicts 6 month outcome in comatose patients with severe traumatic brain injury (TBI). The optimal time for SSEP testing is not known.

Methods: Median SSEP grades ${ }^{1}$ were obtained at the beginning, middle and end of the first week after injury on 31 patients with severe TBI. SSEP grade was statistically compared to 6 month Glasgow Outcome Scale (GOS).

Results: SSEP grade at the beginning $(\mathrm{R}=0.47, \mathrm{p}=0.008)$, middle $(R=0.68, p=0.0001)$ and end of the week $(R=0.6$, $\mathrm{p}=0.0003$ ) related significantly with GOS. One test time was not significantly different than the others in relating to GOS (beginning vs. end, $p=0.4$; middle vs. end, $p=0.8$; beginning vs. end, $\mathrm{p}=0.5$ ). Patients with a beginning intermediate SSEP grade which increased or stayed the same by the end of the week $(n=16)$ had a better prognosis for independent functioning at 6 months than those with SSEP grade deterioration $(n=3)$ all of whom died or remained in a vegetative state.

Conclusions: SSEP grade obtained at the beginning of the first week after injury may be a useful tool in the early clinical management of patients with severe TBI. Patients with intermediate SSEP grade should have serial testing because SSEP grade deterioration within the first week is a poor prognosis for further neurological recovery.

Reference: 1. Houlden DA, Chen Li, Schwartz ML, Katic M: Median nerve somatosensory evoked potentials and the Glasgow Coma Scale as predictors of outcome in comatose patients with head injuries. Neurosurgery 27:701-708, 1990.

\section{H-10}

Percutaneous Balloon Microcompression of the Gasserian Ganglion for Trigeminal Neuralgia

\section{S.S. BAEESA, B.G. BenotT (Ottawa, Ontario)}

Background: A percutaneous method of gasserion ganglion compression, utilizing a balloon catheter, was first described by 
Mullan and Lichtor in 1983. This relatively minor procedure is an attractive alternative in patients with trigeminal neuralgia, who are not candidates for microvascular decompression (MVD).

Methods: During the period 1989 - 1995, 37 balloon procedures were performed on 33 patients. All patients had idiopathic trigeminal neuralgia, except for one with multiple sclerosis. The mean age of the patients was 61 years (range $32-83$ years) and $64 \%$ were females. Ten of the 33 patients presented with pain recurrence after treatment by other surgical techniques.

Under light general anesthesia, with lateral C-arm fluoroscopic monitoring, a $14 \mathrm{~F}$ needle is introduced into the foraminal ovale. A \#3 F Fogarty catheter is passed into Meckel's cave through the needle and inflated with $0.5-0.75 \mathrm{cc}$ of contrast for a mean period of 15 minutes (range $6-20 \mathrm{~min}$.). There were two failed cannulations. Most patients were discharged the day of surgery (31/37) and the mean follow up period was 31 months.

Results: All patients had immediate post-operative relief of pain, and $89 \%$ had mild numbness. There was no anesthesia dolorosa or keratitis. There was one case of aseptic miningitis (uneventful outcome), one case of cardiac asystole upon dural penetration (rhythm restored quickly) and one case of trochlear nerve paresis with recovery after 3 months. Tic recurred in $30 \%$ $(\mathrm{N}=10)$, after a mean interval of 20 months. These patients underwent further procedures; balloon (4), MVD (3), peripheral neurectomy (3). There was no direct correlation between the recurrence of neuralgia or post-operative numbness and duration of microcompression.

Conclusion: Percutaneous balloon microcompression is a simple, safe, and effective option for selected patients with trigeminal neuralgia. Recurrence rates are comparable to other methods, and the procedure can be done on an out-patient basis.

\section{H-11}

Long-Term Results of Microvascular Decompression and Partial Trigeminal Sensory Root Section in the Treatment of Tic Douloureux

\section{G.G. Ferguson, D. BISNAIRE (London, Ontario)}

Background: There is considerable interest in the use of microvascular decompression (MVD) with or without partial trigeminal sensory root section in the treatment of tic douloureux. There has been some skepticism regarding the safety and long-term efficacy of these procedures.

Methods: Since 1977, 133 patients with tic douloureux have undergone 146 posterior fossa procedures; an initial MVD in 106, a partial root section in 30 and re-exploration in 10,9 of whom underwent partial root section. The patients have been followed prospectively. Only 1 patient has been lost to followup.

Results: The male/female ratio was $49 / 84$. The average age at treatment was 55 . There have been no deaths and no patient has suffered significant persisting disability. One patient required reexploration for a postoperative hemorrhage. One patient had a minor pontine infarction. Two patients have mild ataxia, 2 have moderate hearing loss and 3 have somewhat bothersome sensory loss. The follow-up averages 6.6 years ( 30 days to 18.3 years). Based on the results of the initial procedure, $63 \%$ have been totally pain free, $10 \%$ have had minimal pain requiring no treatment, $13 \%$ have required Tegretol at some time, and $14 \%$ have required further surgical treatment.

Conclusions: Eighty-six percent of patients undergoing posterior fossa exploration and either MVD or partial sensory root section as an initial procedure for tic douloureux have required no further surgical treatment when followed for up to 18 years.

\section{PEDIATRIC NEUROSURGERY}

\section{I-01}

Realistic Simple Mathematical Model of Brain Biomechanics for Computer Simulation of Hydrocephalus and Other Brain Abnormalities

J.M. Drake, O. Mostachfi (Toronto, Ontario), G. Tenti, S. Sivaloganathan (Waterloo, Ontario)

Background: The failure of computer simulations to accurately predict the mechanical response of the brain to such processes as hydrocephalus, head injury, and tumors, stems from a lack of a suitable model, and lack of information about brain mechanical properties. We have developed a simple but realistic brain model which allows us to estimate theses properties.

Methods: We assumed that the brain is a porous solid filled by a fluid, a two-phase system. We solved directly the equations for consolidation theory, using simplified brain geometry. Profiles for brain tissue pressure, brain tissue displacement, and brain fluid flow, were generated for chronic compression such as with hydrocephalus. The effects of mechanical properties Young's modulus, shear modulus, Poisson ratio were analyzed.

Results: Solution of this model demonstrates that the response of the brain to mechanical forces is critically dependent on the Poisson ratio, which we estimate to be between .3 and .35 (not .5 as has generally been assumed by others). Brain mechanical response is less sensitive to Young's modulus and shear modulus. We estimate that the shear modulus of normal brain is $5000 \mathrm{~N} / \mathrm{m}^{\wedge} 2$ by recalculating previous measurements by Metz et al. (1979).

Conclusions: This model explains the failure and instability of previous simulations which assumed that the brain is incompressible (Poisson ratio $=.5$ ). Further accurate measurements of the shear modulus are required. Robust and accurate computer simulations will then be possible.

\section{I-02}

Utero Intracranial Hemorrhage as Result of Neonatal Alloimmune Thrombocytopenia

\section{B.S. Jhawar, R.F. Del Maestro, (London, Ontario)}

Background: Neonatal alloimmune thrombocytopenia (NAT) results when maternal antibodies attack fetal platelets canying paternal antigens. 
Method: Two cases of in utero intracranial hemorrhage are reviewed.

Results: Both children were born to mothers with habitual miscarriages. Case 1 occurred in a preterm infant born by cesarean section for premature labour and macrocephaly. Case 2 was an uneventful pregnancy and delivery. Both infants displayed multiple ecchymosis and petechiae at birth with profound thrombocytopenia noted within the first few hours of life. Treatment was with steroids, gamma globulin and platelet transfusion with complete resolution of the platelet deficit. Both infants had intraparenchymal and intraventricular hemorrhage associated with hydrocephalus seen initially by ultrasound and then supported by CT. Initially, each child was managed with serial ventricular taps. Case 1 was shunted at 28 days and three years later showed some global developmental delay with decreased visual acuity. Case 2 was shunted at 15 days and follow up at 7 months showed good developmental progress.

Conclusion: NAT occurs in about $1 / 2000$ live births. It is associated with intracranial hemorrhage in about $15-20 \%$ of cases, with approximately $25 \%$ occurring in utero. Appropriate management consists of early diagnosis, correction of platelet abnormalities and preferable cesarean section. Often intracranial hemorrhage can be managed expectantly. Hydrocephalus is common and ventricular taps may temporize until a definitive VP shunt can be inserted.

\section{I-03}

Dandy-Walker Syndrome: Cerebellar Size and Functional Outcome

\section{A.S.Y. Lee, M. Sargent, P. Steinbok (Vancouver, British Columbia)}

Background: In children with Dandy-Walker malformation the relationship between post-shunting changes in cerebellar size and motor development was examined.

Methods: Children with Dandy-Walker syndrome or DandyWalker variant at this institution were reviewed retrospectively. Initial and followup CT scans were reviewed by a pediatric neuroradiologist blinded to clinical outcome.

Results: 11 patients were reviewed. Cases were initially treated with cystoperitoneal (5) or ventriculoperitoneal (6) shunting. In all cases shunting reduced ventricular size as determined by CT scan; this was independent of shunt type. Cyst size decreased in 9/11 cases; there was a tendency for posterior fossa cyst size to reduce better with cystoperitoneal shunting. There was an increase in cerebellar hemispheric size with 10/11 cases although the vermis rarely increased in size (1/11). Children with normal development had a greater degree of increase in cerebellar size than developmentally-impaired children. Initial shunt selection did not correlate with subsequent cerebellar size.

Conclusions: Cerebellar hemispheric size commonly increases after either ventricular or cystoperitoneal shunting. The least increase in size tends to occur with developmentally-impaired children.

\section{I-04}

Fenestration of Porencephalic Cysts to the Lateral Ventricle: Experience with a New Technique for Treatment of Seizures

C.A. KoCh, J.L. MoOre (Columbus, Ohio), K.H. KRäHLING, D.G. PALM (Münster, Germany)

Background: Unilateral porencephalic cysts are brain cavities resulting from perinatal vascular occlusion, most often in the territory of the middle cerebral artery. Severe neurological deficits such as hemiparesis are commonly associated with porencephalic cysts as well as intractable seizures. Between 1978 and 1992 we treated 37 children with medically intractable epilepsy and porencephalic cysts by uncapping and fenestration of these cysts to the lateral ventricle.

Methods: We retrospectively reviewed all cases and assessed the outcome of these patients with regard to seizures, paresis, and neurosurgical complications.

Results: Of 37 children, $62 \%$ (23/37) were postoperatively seizure-free. Nine patients $(24 \%)$ had a reduction of seizures and 5 children (14\%) remained unchanged. Of 30 patients with preoperative hemiparesis, $30 \%$ (11/30) improved after the operation over a follow-up period of 1 year. The size of the fenestration varying from $0.4 \mathrm{~cm}^{2}$ to $2.0 \mathrm{~cm}^{2} \mathrm{did}$ not influence the seizure or hemiparesis outcome. However, with respect to the postoperative complications a size of $1.0 \mathrm{~cm}^{2}$ is recommended. The leading postoperative problem was a subcutaneous/subgaleal cushion of cerebrospinal fluid (CSF), which affected 12 children (12/37). The treatment consisted of pressure dressings. A dura patch covering the iatrogenic dura defect could not prevent or reduce CSF leakage, but prolonged the postoperative fever period. Postoperative fever occured in 36 children (36/37) and was due to an aseptic meningitis. Nine of 13 postoperative CT-scans of the head showed a reduction of the cyst size along with seizure reduction. Four CT-scans (4/13) revealed an unchanged cyst size in association with seizure reduction.

Conclusion: Children with porencephalic cysts and seizures benefit from uncapping and fenestration of these cysts to the lateral ventricle. Concomitant perioperative complications are not severe and are easy to treat.

\section{$\mathbf{I}-\mathbf{0 5}$}

Results of Posterior Fossa Decompression in Children and Adolescents with Scoliosis, Chiari 1 Malformation, and Syringomyelia

\section{F. Pirouzman, R. Kazim, J. Drake, D. Medden (Toronto, Ontario)}

Background: Scoliosis in children or adolescents associated with syringomyelia and Chiari I malformation is a well recognized entity. Surgical decompression of the Chiari I malformation is an established treatment, but there is little information on the response to surgery.

Methods: A retrospective chart review of all patients presenting with this clinical triad was undertaken. The patients clinical findings were related to the size, extent, location of the syrinx 
and its response to treatment as well as the progression of spinal curvature.

Results: Thirteen patients ranged in age from 9 to 17 years. Most were referred for evaluation of an atypical or rapidly progressive scoliosis, but $76 \%$ had sensory deficits and $38 \%$ had loss of deep tendon reflexes or spasticity. The scoliosis had been followed for an average of 2.6 years prior to syrinx diagnosis. All but two patients had moderately severe scoliosis with Cobb's Angle $>40$ degrees. The syrinxes were large extending from upper cervical to mid thoracic or lumbar segments on presentation. All patients underwent post fossa decompression. Two required syringopleural shunts for persistent syrinxes. Two other patients underwent spinal fusion and instrumentation for advanced scoliosis. The progression of spinal curvature following surgery was slowed or arrested but in general not improved. Likewise there was little improvement in neurological status.

Conclusions: Currently patients with scoliosis, syringomyelia and Chiari I malformation present at a relatively advanced stage with significant neurological deficits. Although the disease process is arrested by treatment, there is little improvement. Earlier recognition of this problem would likely improve patients outcome.

\section{I-06}

\section{A Randomized Clinical Trial to Compare Selective Posterior Rhizotomy Plus Physiotherapy with Physiotherapy Alone in Children with Spastic Diplegic Cerebral Palsy}

P. Steinbok, A.M. Reiner, J.R. Kestle, R.W. Armstrong, R.D. Beauchamp, D.D. Cochrane, R.D. Keyes (Vancouver, British Columbia)

Introduction: Selective posterior rhizotomy (SPR) is frequently done to treat spasticity in children with cerebral palsy, but the value of the operation has been challenged.

Methods: A randomized controlled trial was carried out to determine whether SPR followed by intensive physiotherapy was more effective than intensive physiotherapy alone in improving motor function in children with spastic diplegic cerebral palsy. Patients randomized to the surgical arm $(n=15)$ had surgery within 1 month, and had intensive physiotherapy for 9 months postoperatively. Patients randomized to physiotherapy only $(n=15)$ had identical intensive physiotherapy for 9 months. The primary outcome measure was the change from baseline to 9 months in the Gross Motor Function Measure (GMFM), a quantitative and validated measure for use in children with spastic cerebral palsy.

Results: There was a statistically significant and clinically important improvement in outcome for the children with rhizotomy, with a mean increase in GMFM score at 9 months of $11.3 \%$ for the surgical group compared to $5.2 \%$ for physiotherapy only $(t=3.03, p=0.007)$.

Conclusions: This study shows that selective posterior rhizotomy improves function of children with spastic diplegic cerebral palsy, over and above the improvement that might be obtained by intensive physiotherapy alone, thus validating this surgical procedure.

\section{I-07}

Functional Magnetic Resonance Imaging (fMRI) Assessment of the Relationship Between Eloquent Cortical Function and Cortical Lesions in Pediatric Neurosurgical Patients.

E.T. Kiriakopoulos, M. Bernstein, J. Rutka, J. Drake, H.J. Hoffman, R. Humphreys, H. Otsubo, S.N. Nicosia, M.L. Wood, D.J. Mikulis (Toronto, Ontario)

Purpose: This study was done to determine the utility of fMRI in localizing the proximity of eloquent cortex to cerebral lesions in pediatric neurosurgical patients.

Methods: fMRI was performed using a $1.5 \mathrm{~T}$ conventional system to localize motor, sensory, speech and visual areas in neurosurgical patients $(n=25)$. The age range of this pediatric patient population was 22 months to 18 years. Activation paradigms included finger tapping, light touch sensory stimulation, repetition of days of the week and photic stimulation. Statistical analysis $(\mathrm{p}<0.0001)$ of the functional data consisted of t-test comparisons between resting and task images.

Results: In 23/25 patients fMRI activation maps were produced. Two patients were unable to complete imaging. In 23/23 successful fMRI mappings the relationship between eloquent cortex and lesions was clearly demonstrated and aided in surgical planning. Intraoperative electrocortical stimulation was correlated with fMRI data to examine the reliability of fMRI brain maps.

Conclusions: The collection of accurate data using fMRI paradigms designed for children is demonstrated. We highlight the utility of single slice data in localizing eloquent cortex. This represents the largest single collection of pediatric cases studied with fMRI and includes the youngest reported patient studied, at 22 months of age.

\section{I-08}

Focal Midbrain Gliomas: Long Term Survival in a Cohort of 16 Patients and the Implications for Management

\section{M.G. Hamilton, C. Lauryssen, N. Hagen (Calgary, Alberta)}

Background: Focal gliomas involving the midbrain tectum, and more recently the tegmentum have been identified as having a better prognosis than diffuse tumors affecting the brain stem. However, only limited information is available concerning treatment effectiveness and long term outcome for these patients.

Methods: A retrospective, population-based cancer registry survey was performed to assess the clinical features and treatment courses of patients with focal midbrain tumors.

Result: Sixteen patients with midbrain gliomas were identified; eight had tectal gliomas and eight tegmental gliomas. Thirteen patients presented with symptoms related to hydrocephalus, and 12 required a VP shunt. Seven patients underwent surgery directed at the tumor. Eight patients underwent radiation therapy and none had initial chemotherapy. One patient diagnosed at age 18 months had a rapidly growing tumor after 14 months of follow up which has responded to chemotherapy. The mean survival of this patient 
population was 84 months (range 3-280 months) after diagnosis, with only one tumor related death occurring ( 280 months after diagnosis). Survival was not affected by tumor location within the midbrain (tegmental or tectal; $\mathrm{p}=0.11$ ) or by whether radiation therapy was or was not administered $(\mathrm{p}=0.36)$.

Conclusions: We conclude that patients with focal midbrain gliomas require symptom control aimed at treatment of hydrocephalus, or mass effect from the tumor. However the extended survival of this population suggests that aggressive surgical debulking is often not required. Furthermore, the routine use of radiation or chemotherapy for all such patients is also questioned.

\section{I-09}

\section{Endocrine Outcome in Children with Brain Tumours}

\section{R.L. Del Maestro, C.L. Clarson (London, Ontario)}

Background: Primary brain tumours (BT) are the most common solid tumours of childhood. Survival rates of these children have improved dramatically in the last 15-20 years due to more aggressive treatment. Long term sequelae of radiation and chemotherapy include endocrine dysfunction.

Methods: Growth and pubertal development were monitored prospectively from within 1 year of diagnosis of a nonpituitary related BT in 41 children aged 2.3 - 13.8 yrs. Provocative growth hormone $(\mathrm{GH})$ testing was performed if the annual height velocity (HV) was below the 25th percentile for age at 2 years or more from completion of BT therapy. Data from 7 children were excluded from the growth analysis; 6 who were within $10 \mathrm{~cm}$ of their target height at diagnosis and 1 with BT recurrence. Serum TSH, Free T4 and fasting cortisol were measured annually.

\section{Results:}

1. GH deficiency was confirmed in 14 of $34(41 \%)$ prepubertal children, all of whom were treated with cranial irradiation. 12 of 13 (92\%) with medulloblastoma (MBA) and 2 of 21 with other BT diagnoses.

2. Precocious puberty was documented in 2 of 34 (5.9\%) children.

3. 3.5 of $41(12.2 \%)$ children (all with MBA) had primary hypothyroidism but none had central hypothyroidism.

4. ACTH deficiency was not documented in this group.

Conclusions:

1. GH deficiency and primary hypothyroidism are common following cranial irradiation for nonpituitary related BT particularly MBA.

2. These findings reflect the need for prospective endocrine monitoring of children with BT's.

\section{I-10}

\section{Cavernous Angiomas in Childhood: Ste-Justine Hospital Experience over 10 years}

\section{H. Mclelland, A. Turmel, C. Mercier (Montreal, Quebec)}

Cavernous angiomas are vascular malformations consisting of caverns lined by endothelial cells and collagen filled with blood degradation products at different stages. With imaging improvement, their diagnosis is easier and more frequent; however, in childhood, their presentation is known to be infrequent.

We present our pediatric experience since 1985 with 11 children with cavernous angiomas located at the following sites: lobar (5), brainstem (2), cerebellum (1), cervical intramedullary (1), thoracic epidural (1), conus medullaris (1). Patients presented with focal deficits (7), seizures (3) and one case discovered during headache workup. Surgical diagnosis was made in 8 cases. In the remaining 3 patients, appropriate radiologic follow-up was carried out. We examined the clinical, radiologic, pathologic findings, management and follow-up of those patients associated with a literature review.

Infrequently seen in the pediatric population, cavernous angiomas can be observed in a broad spectrum of location in this era of magnetic resonance imaging. They represent challenging clinical entities with their controversial management combined with the long life expectancy of children.

\section{I-11}

Surgical Management of Simple Craniosynostosis at the Children's Hospital of Eastern Ontario, Ottawa, Canada

\author{
V.K. Srivastava, M.A. Alonso-Vanegas, and C.H. Lam \\ (Montreal, Quebec) M.J. Higgins and E.C.G. Ventureyra \\ (Ottawa, Ontario)
}

Background: Craniosynostosis is characterized by premature sutural fusion. The etiology of this process is unknown but is probably due to an inherent mesenchymal bone defect. The type of cranial deformity depends on which sutures are involved.

Methods: From 1974 to 1994, 335 cases of craniosynostosis were surgically managed. We identified 88 patients $(26 \%)$ operated for scaphocephaly and 36 patients (11\%) for trigonocephaly. The age distribution ranged from 6 months to 2 years; most of the patients were operated at the younger ages. There was a male predominance of $85 \%$. The main surgical indication was cosmetic and the operative intervention was directed toward releasing the affected suture. Preoperatively, all patients underwent clinical assessment, craniometry, skull radiographs, CT, 3D-CT and MRI.

Results: The surgical outcome was classified as excellent, good or poor according to the clinician and the parents, with 95\% corresponding to the first two categories. More recently, the average hospital stay was 48 hours. There was no mortality in this series and only minor morbidity.

Conclusions: As the most recent reported series of craniosynostosis, a trend of shortened hospitalization, decreased morbidity and good surgical outcome with low incidence of reoperation were found. 


\section{POSTER PRESENTATIONS}

\author{
SESSION 1 \\ Basic Neurophysiology
}

\section{P-001}

\section{Expert System Approach To Analyze EEG Signals}

\section{S.S. SESHIA, L. YAO, E. SHWEDYK, (Winnipeg, Manitoba)}

Computer aided analyses facilitate the interpretation of prolonged EEGs. Current approaches are based on traditional signal processing methods. An expert system is intuitively appealing because it "mimics" clinical neurophysiologists. We describe the principles of such a system for analyzing background and sharp waves (Sts).

Epochs (20 seconds each) were selected visually from EEGs done, using a bipolar montage, on a 16 channel ambulatory recorder. Analysis rules developed by a clinical neurophysiologist using accepted approaches, were programmed on a MacIntosh platform. $C$ language was used to extract appropriate features which were then interpreted by expert software programmed with CLIPS. Independent training $(\mathrm{N}=40)$ and evaluation ( $\mathrm{N}=40$ for STs; $\mathrm{N}=10$ for background) epochs were used. The performance of the system was compared with that of the clinical neurophysiologist. The system detected 209 STs "rejecting" 7 as artifact. The false negative rate for ST "detection" was $9 \%$. The false positive rate was $5 \%$ to $66 \%$, depending on the definition used. The consistency in interpreting background was delta $(79 \%)$, theta $(97 \%)$, alpha $(67 \%)$, beta $(20 \%)$. We suggest that expert system methods may have a useful role in analyzing EEG signals.

Funded by Health \& Welfare Canada \& HSCRF

\section{P-002}

\section{Photoperiodic Entrainment and Alpha Rhythm: New Approach to an Old Question}

\section{M.A. CORTEZ (Toronto, Ontario), X.A. CASTRO (Quito, Ecuador)}

Background: Alpha rhythm frequency increases from $4 \mathrm{~Hz}$ at four months after (term) birth, to $8 \mathrm{~Hz}$ at three years of age in visually intact infants, and never develops in congenitally blind patients. The biological significance of the alpha rhythm is unknown.

Methods: Environmental Light-Dark (L-D) cycle data (1 cycle/24 Hours) was plotted 1095 times (3 years), against the maturational curve of the alpha rhythm frequency (Kellaway P.), to determine the environmental light / alpha frequency ratio (r).

Results: Photoperiodic data produced a computerized oscillation comparable to the EEG signal. 121.68 L-D cycles preceded $4 \mathrm{~Hz}$ activity ( $r=1: 30.42)(0.032) ; 243.36 \mathrm{~L}-\mathrm{D}$ cycles lead to the 5th and 6th Hz output $(\mathrm{r}=1: 121.68)(0.008) ; 730.08 \mathrm{~L}-\mathrm{D}$ cycles to approach the 7 th. and the 8 th $\mathrm{Hz}(\mathrm{r}=1: 365.04)(0.002)$. (Mean $=136.87$ L-D cycles), $\mathrm{Chi}^{2}=82.79$ (p.<0.0002).

Conclusion: The L-D cycle/ alpha rate is 8 times faster in the initial $4 \mathrm{~Hz}$ compared to the following $4 \mathrm{~Hz}$ of the alpha rhythm, consistent with the maturational process, however the evolving oscillatory aspect of the alpha rhythm suggests a critical photoperiodic oscillation entrainment on alpha generators.

\section{P-003}

\section{Pain Thresholds are Modified by Autonomic Balance}

A.R.M. Upton, G. Tougas, M. Kamath, R.M. Spaziani, V. Dzuric, D. Armstrong, B. Clarke, E. Fallen (Hamilton, Ontario)

Background: Direct left vagal stimulation in 10 patients with intractable seizures allowed validation of the parasympathetic origin of the high frequency component (HF 0.15-0.5 Hz) of power spectral analysis of heart rate variability (PS/HRV). PS/HRV was used to assess the autonomic balance of the sympathetic low frequency component (LF $0.05-0.15 \mathrm{~Hz}$ ) and the parasympathetic HF component during acid administration (0.1 $\mathrm{N} \mathrm{HCL}, 10 \mathrm{ml} / \mathrm{min} 10 \mathrm{~min}$ ) while monitoring subjective pain responses.

Methods: 10 healthy male volunteers $(26.5 \pm 1.9$ yrs.) and 28 consecutive patients $(40 \pm 10$ yrs. $13 \mathrm{~F})$ received ECG monitoring 20 minutes before and during oesophageal acid perfusion. The $\mathrm{HF}$ and LF components of the HRV were measured using autoregressive modeling. The pain response was recorded and compared with the autonomic (LF/HF) balance.

Results: All 10 volunteers and $9 / 28$ patients $(4 \mathrm{~F})$ had no symptoms during acid infusion. 19 patients had symptoms. A stepwise discriminate analysis of heart rate and PS/HRV indices predicted pain responses to acid secretion in $25 / 28$ patients $(85 \%)$ with a specificity of $84 \%$ and a sensitivity of $100 \%$ $(p<.05)$. The LF:HF power ratio decreased by $24 \%(2.61 \pm 0.25$ at rest to $2.11 \pm 16, \mathrm{p}<.05$ ) in patients with pain during acid perfusion but not in asymptomatic patients or controls.

Conclusions: The autonomic balance and decreased resting vagal tone correlate with pain responses to acid perfusion in the oesophagus.

Acknowledgements: We are grateful to the DeGroote Foundation and MRC Canada for their support.

\section{P-004}

Hippocampal Slices Perfused with Sub-convulsant Doses of Both 4-aminopyridine and Penicillin: An Interictal EEG Model?

\section{J.H. SCHNEIDERMAN (Toronto, Ontario)}

Interictal EEGs in patients with intractable temporal lobe epilepsy are normal or demonstrate occasional spikes intermingled with normal or slow background activity. These electrophysiologic changes are not well reproduced by in vitro models currently used to investigate seizure mechanisms. We examined the interaction between sub-convulsant doses of penicillin $\left(\mathrm{GABA}_{\mathrm{A}}\right.$ antagonist) and 4-aminopyridine (4-AP) $\left(\mathrm{K}^{+}\right.$channel blocker) in hippocampal slices in order to develop an interictal EEG model based on multiple, subtle pathophysiologic changes. 
Low concentrations of 4-AP $(10 \mu \mathrm{M})$ increased the amplitude of $\mathrm{CA}_{3}$ spontaneous field potentials without altering their frequency distribution but did not induce the spontaneous bursting seen with high concentrations $(50 \mu \mathrm{M})$. Sub-convulsant doses of penicillin (50-300 IU/ml) produced low amplitude, low frequency $(2-4 \mathrm{~Hz})$ oscillations and supressed high frequencies $(>8-10$ $\mathrm{Hz}$ ). Partially synchronized spontaneous bursts characterized by long, variable intervals and variable amplitudes were intermingled with low frequency oscillations in slices perfused with low doses of both penicillin and 4-AP. Low frequency stimulation was usually required to initiate spontaneous bursting.

Conclusions: Combining low doses of two mechanistically different convulsants produces partially synchronized bursts and low frequency oscillations closely resembling interictal EEG activity in patients with temporal lobe epilepsy.

\section{P-005}

\section{Apoptosis in Mouse Hippocampus After Intraperitoneal} Administration of Domoic Acid

P.J.S. Vig, M. Sundaram, D. Desaiah and J.D. Fratkin (Jackson, Mississippi)

Background: Domoic acid (DA) is known to cause neuronal damage in the hippocampus. However, the mechanisms governing this process are not fully understood. In this report we studied whether excitotoxic neuronal death evoked by DA in mouse hippocampus is associated with apoptosis.

Methods: Male ICR mice were divided into two groups. Group I $(n=4)$ animals were given a single dose of DA $[5 \mathrm{mg} / \mathrm{kg}$ intraperitoneally (ip)] and group II $(n=4)$ animals received normal saline. The animals were sacrificed after $24 \mathrm{hr}$. The brains were quickly removed and processed for in situ terminal-transferase-mediated nucleotide-digoxigenin nick end labeling. The labeled cells were visualized by reaction with anti-digoxigenin antibody conjugated peroxidase and a suitable substrate.

Results: Sections from the hippocampus of DA treated animals showed a considerable number of apoptotic cells in hilus of dentate gyrus. Other areas of a hippocampus had only a few apoptotic cells, whereas in cerebellum, and frontal and temporal cortex no apoptotic cells were seen in DA treated animals.

Conclusion: Our results suggest that DA excitotoxicity is associated with apoptotic cell damage in hilus of dentate gyrus.

\section{P.006}

\section{The Functional Organization of Dysgenic Cortex}

R. Leblanc, M. Preul, J.G. Villemure, D. Reutens, E. Meyer (Montreal, Quebec)

Background: Cortical dysplasias (CD) are of interest because of their relationship to epilepsy. These lesions can be independently epileptogenic and their inclusion within the resected area largely determines the degree of seizure control in operated patients. Although much is known of the embryogenesis, morphology and ultrastructure of $\mathrm{CD}$ their physiology remains largely uncharted.

Methods: We describe the results of cortical stimulation, of functional Positron Emission Tomography (f-PET) and of Magnetic Resonance Spectroscopy (MRS) in 3 patients with CD operated on for the treatment of medically intractable epilepsy. The 3 patients ( 2 males and 1 female) aged 16 to 45 years had temporal lobe ( 2 cases) and frontal lobe (1 case) epilepsy. The areas of $\mathrm{CD}$ were in the ipsilateral central region in 2 cases and in the language dominant temporal lobe in the other.

Results: Cortical mapping of the dysplastic areas produced motor responses when the $C D$ involved the precentral gyrus and interference with language when the $C D$ was in the language dominant posterior temporal lobe. The homuncular pattern was grossly distorted in the patient with the most severe CD. f-PET corroborated the results of cortical stimulation. PET after the infusion of deoxyglucose revealed preserved glucose metabolism deep in the white matter in the dysplastic area, similar to that seen in normal cortex. MRS suggested a shift towards anaerobic metabolism.

Conclusions: Dysplastic cortex can retain motor and language function. Cortical mapping should be performed prior to its resection if the $C D$ resides within a putatively functional area such as the pre- or post-central gyrus and the speech regions. The degree of functional organization is inversely proportional to the severity of the $\mathrm{CD}$. The dysplastic area may remain functional by a shift to anaerobic glucose metabolism.

\section{P-007}

\section{Epileptiform Activity Recorded from Isolated Cortex} Following Functional Hemispherectomy

R. WennBerG, F. Quesney, J.G. VILLEmURe (Montreal, Quebec)

Background: Electrocorticography (ECOG) performed before and after functional hemispherectomy (FH) allowed for examination of the effect of cortical isolation upon interictal epileptiform activity (EA).

Methods: Pre- and post-excisional ECOG was performed on 16 patients with hemiparesis and intractable epilepsy who underwent FH between 1987 and 1993. Presence or absence and relative abundance of post-excisional EA recorded from isolated parietal-occipital and frontal cortices was analyzed.

Results: 7 cases had pre-excisional EA recorded independently from frontal and parietal-occipital cortices: all 7 had EA recorded from isolated frontal and parietal-occipital cortices after FH. 2 cases had EA restricted to frontal cortex before and after FH. 2 cases with no pre-excisional frontal recording had parietal-occipital EA recorded before and after FH. 5 cases showed no frontal or parietal-occipital EA before FH: 3 of these showed no EA after FH, 1 showed EA restricted to isolated parietal-occipital cortex, and 1 had EA recorded from isolated frontal and parietal-occipital cortices. In total, 30 disconnected cortices were recorded from: relative abundance of EA was increased in 10, unchanged in 17, and decreased in 3 .

Conclusions: EA recorded from isolated cortex is representative of the pre-excisional epileptogenic state. Increase or appearance of EA following disconnection may represent a decreased threshold for EA expression in isolated cortex. 
Postural Stability during Chronic Left Vagal Nerve Stimulation

B.M. Clarke, A.R.M. Upton, H. Griffin, M. Denardis, D. FitzPATRICK (Hamilton, Ontario)

Background: Stimulation of the left vagus nerve (VNS) has been shown to reduce seizures in double blinded crossover studies in man. Animal studies have reported autonomic nervous system (ANS) effects on nociceptive and motor reflexes. Anticonvulsant drug therapy may cause postural instability over time. Long term effects ( 2 1/2 years) of left vagal nerve stimulation included assessment of postural stability analysis.

Methods: 6 epileptic subjects, 5 males and 1 female, mean age $33 \pm 9.16$ years, with intractable complex partial seizures taking 3 anticonvulsant drugs were assessed for postural stability in quiet standing and while moving forwards, backwards and sideways with eyes open and closed. Data were acquired using an AMTI Biomechanics immovable forceplate (Newton, M.A., U.S.A). Data were collected pre-operatively (D1) and at 6-8 weeks (DI-D3) and every 6 months thereafter (D4-D6).

Results: $4 / 8$ balance measures were worse over time $(\mathrm{p}=$ $.022, p=.003)$. In one case $(p=.019, p=.004)$ with eyes closed, there was improvement in area of sway. There were no significant ON/OFF effects on posture.

Conclusion: Chronic left VNS does not produce significant changes on postural instability. Deterioration over time is probably due to drug effects and the natural history of epilepsy.

\section{P-009}

\section{Central and Peripheral Visual Field Pattern Evoked Response Latencies}

\section{D.C. Jones, W.T. Blume (London, Ontario)}

Background: Whether fibres subserving the peripheral visual fields conduct impulses more rapidly than those subserving macular vision to orientate or "set" the subject for specific visual information is studied, seeking an analogy with the motor system in which conduction of the extrapyramidal system is more rapid than the pyramidal one.

Method: Averaged responses evoked by pattern stimuli sequentially mixed between central and peripheral fields to assure constancy of state were referentially recorded by a $3 \times 5$ channel grid centred $5 \mathrm{~cm}$ above the inion in humans. Only averaged potentials with a clearly defined NPN complex were included in this analysis.

Results: Histogram of the N75 and P100 peaks revealed peak latencies to peripheral stimuli to occur $4 \mathrm{msec}$ earlier than to central stimuli (P100:102 and $106 \mathrm{msec}$ ).

Conclusion: These results suggest that, in the human, conduction of fibres from $M$ retinal cells, responding to gross features of large objects, is more rapid than that of $\mathrm{P}$ retinal cells whose receptive cells are smaller and convey fine detail. The more rapid conduction of the former type would "set" the visual apparatus for reception of finer detail.
Cognitive Motor Strategies after Chronic Stimulation of the Left Vagus Nerve

B.M. Clarke, A.R.M. Upton, H. Griffin, M. Denardis, D. FITZPATRICK (Hamilton, Ontario)

Background: Left vagal nerve stimulation (VNS) has been shown to control seizures in double blinded crossover studies in man. Cognitive decline occurs in chronic epileptics on medication. VNS does not augment this decline. The effects of a visual cue on cognitive motor strategies (CMS) was assessed.

Methods: 6 epileptic subjects, 5 males and 1 female, mean ages $33 \pm 9.16$ years with intractable complex partial seizures taking three anticonvulsant drugs were assessed for CMS over a 28 month period. Subjects responded using an electronic switch pad to a cued signal appearing on an Apple Il E computer screen. The test determined how much the subject relied on the cue (cost-benefit analysis).

Data Analysis: 6 repeated measures of covariance technique with 2 within subject factors, day, and time of day were done.

Results: No significant effect was found for the use of a cue. Raw data analysis showed that the use of the cue did not improve CMS and in some instances was a hinderance.

Conclusion: The use of a visual cue does not augment CMS in subjects receiving chronic vagal nerve stimulation.

\section{P-011}

\section{Magnitude of Changes in SEP during Scoliosis Surgery}

H. Darwish, I. Huber, L. Alfaro, P. Diadori, R. Dewar, J. HARDER, (Calgary, Alberta)

Background: The value of intraoperative SEP monitoring during spinal instrumentation for scoliosis has been established. Difficulties occur when the cortical responses are abolished even when halogenated anaesthetic agents are avoided. Further changes occur during spinal column distraction. We established a protocol for a prospective study of the magnitude of these changes with the following hypothesis: if the change is due to anaesthetic regime, the effect on the median evoked response should be congruent with that occurring in the posterior tibial evoked response.

Methods: We test the children in the awake (preop) state, then obtain a baseline under anaesthesia and then during and after the spinal distraction. The children are examined and the SEP repeated within one year after the operation.

Results: So far 17 children (age 13.7 \pm 4 yrs) have been monitored using this protocol and the same anaesthetic regime. No changes in latency were noted. Significant changes did occur in amplitudes of the median evoked $\mathrm{N}_{19}-\mathrm{P}_{22}$ complex, and the posterior tibial evoked $\mathrm{P}_{37}-\mathrm{N}_{45}$ complex. The preoperative amplitude was $4.56 \pm 1.9 \mu \mathrm{V}$ for $\mathrm{N}_{19}-\mathrm{P}_{22}$ and $4 \pm 2.7 \mu \mathrm{V}$ for $\mathrm{P}_{37}-\mathrm{N}_{45}$. The baseline after anaesthesia was $2.3 \pm 1.35 \mu \mathrm{V}$ for $\mathrm{N}_{19}-\mathrm{P}_{22}$ and $0.97 \pm 0.6 \mu \mathrm{V}$ for $\mathrm{P}_{37}-\mathrm{N}_{45}$. In 6 children the drop was congruent. In 8 , a significantly greater drop occurred in the $\mathrm{P}_{37}-\mathrm{N}_{45}$ amplitude. In 3 , the decrement was greater in $\mathrm{N}_{19}-\mathrm{P}_{22}$. In comparing the drop in amplitude after distraction to baseline an average $52 \%$ change 
occurred in $\mathrm{P}_{37}-\mathrm{N}_{45}$ amplitude. In 2 children the response was abolished transiently, in one predistraction, and in the other postdistraction. Recovery occurred spontaneously within 20 minutes.

Conclusions: We conclude that the anaesthetic suppression of $\mathrm{P}_{37}-\mathrm{N}_{45}$ is greater than for $\mathrm{N}_{19}-\mathrm{P}_{22}$. Spinal column distraction produces further dramatic decreases in $\mathrm{P}_{37}-\mathrm{N}_{45}$ amplitude. If a replicable response can still be elicited or when the change is transient within 20 minutes significant clinical deficits have not occurred in this series to date.

\section{P-012}

\section{Narcoleptic Diagnostic Myths}

\section{E.B. CAHOON (Toronto, Ontario)}

The Guideline Myth: Sleep Attacks 100\%,Cataplexy 100\%, SOREMPS $100 \%$ on Polysomnography or MSLT Testing.

Other conditions excluded from Narcolepsy. ADD, Insomnia, Hypersomnia, Sleep Apnoea, Dreams, Hypnopompic Nightmares, Panic Attacks, Phobias, Alcoholism, Drug Abuse, no Cataplexy, \& no Sleep Attacks.

Treatment: No one must get alerting drugs, appetite suppressants, benzodiazepenes, or any other drugs not in amounts set out by obsessive worriers about numbers of pills per day, or dependency to medication.

These will be discussed with charts showing the occurrence of many of the same symptoms present, as are found in the Tetrad of symptoms of the past (BG-Before Guidelines).

\section{NEUROSURGERY GENERAL}

\section{P-013}

\section{An Association of Canadian Neurosurgery Residents: Some Views}

\section{J. Siddigi, P. Pahapill, A. Ranger, B. Jhawar, M. Abdulhak} (London, Ontario)

Though contemplated at various times, an association of Canadian neurosurgery residents has never been formally constituted. In the present atmosphere of fiscal constraints, absence of a general license prior to completing residency, continuing braindrain of new neurosurgery graduates, and significant fellowship exam failure rates, Canadian neurosurgery residents have special concerns about the future of their specialty and their place in it. We present the results of a cross-country questionnaire on the relevance of a national neurosurgery resident organization, and on the form such a body should take if it is constituted.

\section{P-014}

Neurosurgical Residents vs. Residents from Other Surgical Specialties: A Comparison of In Training Evaluation Report Scores

L. Hodson; G. Zahariadis; R. Cohen; G. Wu; M. Cusimano (Toronto, Ontario)
Background: The accurate and just assessment of clinical competence (CC) is important for faculty, surgical residents and patients alike. Several subjective aspects of surgical residents' (SR) CC cannot be adequately measured by written examinations. The In-Training Evaluation Report (ITER) is an inexpensive, unobtrusive evaluation tool which provides the potential for immediate and constructive feedback. The goal of this research is to compare ITER scores given NR with those given residents from other surgical specialties.

Methods: 188 ITER for 36 NR (mean 5.2 ITER/NR) were compared with 1344 ITER for $199 \mathrm{SR}$ (mean = $6.8 \mathrm{ITER} / \mathrm{SR}$ ) from a cross-section of 7 other surgical specialties. The data set encompasses all ITER for SR enrolled in the surgical program at the University of Toronto for the past 6 years.

Results: Statistical analysis indicates that NR receive higher scores on the ITER than do their counterparts in general surgery (GS), orthopaedic surgery (OS) and urology (U) $(\mathrm{p}<0.05)$. SR scores differ based on the evaluator (EV).

Conclusions: NR fare better on the ITER than their peers in GS, OS and U. The high scores given NR likely reflect EV reluctance to use the lower end of the scale. A low score should thus "flag" worrisome NR. The scale is unable to discriminate "exceptional" from "average" NR

\section{P-015}

Role of Calpain in the Pathophysiology of Secondary Injury Following Trauma to the Spinal Cord

\section{P.A. SChumacher and M.G. Fehlings (Toronto, Ontario)}

Background: During the first few hours following traumatic spinal cord injury (SCI), secondary biochemical events cause further tissue damage, and may represent an important potential site for therapeutic intervention. A rise in intraneuronal $\left[\mathrm{Ca}^{2+}\right]$ is known to contribute to secondary injury following $\mathrm{SCI}$, possibly through activation of the $\mathrm{Ca}^{2+}$-activated protease calpain, and proteolytic degradation of cytoskeletal calpain substrates.

Methods: Following laminectomy at T7, a 35 gram compressive injury was applied to the rat spinal cord for one minute, and animals were sacrificed at the following times postinjury: 15 min, 1 hour, 2, 4, 8, 16, and 24 hours. An S $^{35}$ - labelled cRNA probe for calpain II- a ubiquitously expressed isoform of calpain- was synthesized and used to screen tissue for calpain 11 expression. Calpain activation and NF200 degradation were assessed by western blotting and immunohistochemical staining.

Results: In situ hybridization studies confirm the expression of calpain II mRNA in normal spinal cord grey matter. Using an antibody specific for spectrin proteolytic fragments generated by calpain-mediated proteolysis, we have demonstrated low calpain activity in uninjured spinal cord, with a progressive activation of calpain postinjury to a peak at $1-2$ hours. NF200 undergoes significant degradation within $1-2$ hours. Immunofluorescent staining revealed a loss of NF200-positive axons in dorsal columns, ventral, and lateral funiculi within the same time period postinjury.

Conclusions: The time course of calpain activation closely mirrors that of NF200 degradation. The efficacy of pharmaco- 
logical calpain inhibition for sparing cytoskeletal proteins and improving funtional recovery following SCI is currently being investigated.

\section{P-016}

Present Somatosensory Evoked Potentials in Complete Acute Traumatic Spinal Cord Injury: An Effect of Pharmacotherapy?

\section{K.A. KletTKe, D.A. Houlden, D.W. Rowed (Toronto, Ontario)}

Background: In the two decades that somatosensory evoked potentials (SEP) of lower limb nerves have been used to evaluate acute traumatic spinal cord injury (ATSCI), most studies have demonstrated that the cortical response is absent in all neurologically complete patients. Experimental studies have shown that high-dose methylprednisolone (MP) salvages evoked potential activity that is lost in untreated ATSCI animals.

Methods: Since pharmacotherapy for ATSCl began at our institution (MP or tirilizad mesylate/MP trial), left then right posterior tibial nerve SEP, were performed on 14 complete ATSCI patients without head injury (range 1-11 days, mean 5 days post-injury). Injury levels were from C5-T11 (8 cervical, 6 thoracic).

Results: In 3/14 (22\%) patients the SEP, were deemed unreliable (peripheral neuropathy in one, excessive EMG artifact in two). In $9 / 14$ (64\%) the SEP was absent while $2 / 14$ (14\%) showed present SEP ( 3 and 4 days post-injury), both of whom received a MP bolus and were entered into a tirilizad mesylate/MP trial. Of 11 patients with reliable SEP, 2 died (SEP absent in both), while the remaining 9 patients were all complete $>6$ months post-injury.

Conclusions: The presence of SEP in complete ATSCI represents a change in our previous experience in which all complete ATSCI had absent SEP. For institutions using SEP to investigate ATSCI it may be important to bear in mind the possible effect of pharmacotherapy when interpreting SEP.

\section{P-017}

The "Fast" Potassium Channel Contributes to Posttraumatic Axonal Dysfunction After Acute Spinal Cord Injury

\section{R. NASHMi, M.G. Fehlings (Toronto, Ontario)}

Background: Acute spinal cord injury (SCI) spares a subpopulation of axons in the subpial rim which display dysfunctional conduction properties. After chronic SCI, blockade of "fast" $\mathrm{K}^{+}$ channels with 4-AP improves axonal conduction. However, the role of $\mathrm{K}^{+}$channels in mediating axonal dysfunction after acute $\mathrm{SCI}$ has not been established. Accordingly, in the following investigation we studied changes in the pharmacological sensitivity of dorsal column white matter to a variety of $\mathrm{K}^{+}$channel blockers, including 4-AP, $\alpha$-DTx, a more selective blocker of the "fast" $\mathrm{K}^{+}$channel, TEA, a blocker of the "slow" $\mathrm{K}^{+}$channel, and $\mathrm{CsCl}$, a blocker of the inward rectifier (IR), following acute $\mathrm{SCI}$.
Methods: Studies were conducted on isolated adult rat dorsal columns in vitro $(\mathrm{n}=62)$. Compound action potentials (CAPs) were recorded extracellularly with microelectodes and by the sucrose gap recording technique. Compressive injury was inflicted in vitro with a modified aneurysm clip (closing force $2.0 \mathrm{~g}$ applied for $15 \mathrm{sec}$ ).

Results: Infusion of 4-AP ( 1 and $5 \mathrm{mM}$ ) and $\alpha$-DTx (500 $\mathrm{nM}$ ) resulted in a significantly greater increase in P2 amplitude of injured as compared to noninjured axons for the microelectode derived field potential recordings. Sucrose gap recordings showed a significant increase in CAP area to $(205.3 \pm 9.9 \%)$ and latency $(106.8 \pm 2.8 \%)$ of injured axons with $1 \mathrm{mM} 4$-AP. TEA $(0.1 \mathrm{mM}$ and $10 \mathrm{mM})$, when infused alone and with $\mathrm{CsCl}(10$ $\mathrm{mM}$ ), produced similar effects on injured and intact axons.

Conclusions: The results of this study suggest that the "fast" $\mathrm{K}^{+}$channel sensitive to A-AP and $\alpha$-DTx displays abnormal activity following compressive $\mathrm{SCI}$. In contrast, the TEA-sensitive "slow" $\mathrm{K}^{+}$channel and the $\mathrm{CsCl}$-sensitive IR channel do not appear to contribute to the dysfunctional conduction properties of injured axons.

\section{P-018}

Role of NMDA and Non-NMDA Receptors in Axonal Injury After Spinal Cord Trauma

\section{S. AGRaWal, M.G. Fehlings (Toronto, Ontario)}

Background: Rises in intracellular calcium $\left(\mathrm{Ca}^{+}\right)$have been linked to the pathophysiology of traumatic spinal cord injury and may involve the activation of ionotropic glutamate receptors. Accordingly, we studied the role of NMDA and nonNMDA ionotropic glutamate receptors in traumatic spinal cord injury.

Methods: A strip of adult rat dorsal column $(n=56)$ was pinned in a recording chamber which was perfused with oxygenated Ringer. Compound action potentials were recorded extracellularly from microelectrodes. Injury was accomplished by compressing the dorsal column strip in vitro with a $2 \mathrm{~g}$ clip for 15 sec.

Results: Under control conditions (Ringers), the CAP decreased to $71.4 \pm 2.0 \%$ of preinjury values after a clip compression injury. Blockade of ionotropic glutamate receptors with a combination of the NMDA receptor blocker APV $(50 \mu \mathrm{M})$ and the AMPA/Kainate receptor blocker CNQX (10 $\mu \mathrm{M})$ conferred significant neuroprotection $(91.5 \pm 6.4 \%$ of control values). In contrast application of the NMDA receptor antagonist APV $(50 \mu \mathrm{M})$ did not improve postinjury recovery. Moreover, administration of NMDA $(10 \mu \mathrm{M})$ did not adversely affect recovery of CAP amplitude of the compression trauma. Application of NBQX $(10 \mu \mathrm{M})$, potent AMPA/Kainate receptor blockers enhanced the recovery of CAP amplitude to $85.6 \pm 2.7 \%$ of control values. Administration of the selective agonists AMPA $(100 \mu \mathrm{M})$ or Kainic acid $(100 \mu \mathrm{M})$ resulted in significantly poorer recovery of CAP amplitude to $(59.4 \pm 1.3 \%$ and $59.0 \pm 1.6 \%)$ respectively as compared to control.

Conclusions: In summary, the injurious effects of $\mathrm{Na}^{+}$or 
$\mathrm{Ca}^{+}{ }^{+}$in traumatically injured CNS white matter is in part mediated by the activation of non-NMDA AMPA/Kainate receptors. In contrast, NMDA receptors do not appear to contribute to traumatic CNS white matter injury.

\section{P-019}

EEG and Clinical Associations with Mortality in Comatose Patients in the General Intensive Care Unit.

\section{G.B. Young, J.H. Kreeft, R.S. McLachlan, F.S. Rutledge,} J.D. Demelo (London, Ontario)

Background: Most intensive care units use the APACHE scoring system, which does not incorporate neuro-physiological findings, for prognostic purposes. For specific etiologies, some EEG patterns are associated with favorable and others with unfavorable outcomes. We wished to determine the association of EEG patterns and clinical factors with mortality in comatose patients in the general ICU.

Methods: We reviewed the EEGs and hospital files of all comatose patients in our ICU who had EEGs over the past 10 years. The following were examined separately and in combination for their association with death without recovery of consciousness: EEG classification, EEG reactivity, etiology of coma, age, sex and integrity of brainstem reflexes.

Results: Of 214 patients, the following individual factors or factor combinations had positive predictive values of $>0.80$ and odds ratios of $>2.0$ for mortality: age $>65$ years, anoxia, generalized EEG suppression, absent EEG reactivity, anoxia with partial cranial nerve areflexia, anoxia with EEG suppression, and anoxia with generalized epileptiform activity.

Conclusions: It appears justified to add neurological and EEG findings to the APACHE scoring system, to assess their added value in predicting mortality.

\section{P-020}

CSF Glutamate in Patients with Deteriorating Somatosensory Evoked Potentials After Severe Human Traumatic Brain Injury and Secondary Ischaemia

\section{J.I.M. Brown, A.J. BAKER, R.J. Moulton (Toronto, Ontario)}

Background: Glutamate excitotoxicity is an important putative mechanism of ongoing damage after traumatic brain injury (TBI) and cerebral ischaemia. Ischaemia is an important cause of secondary injury after TBI. It is possible that glutamate release occurs during periods of ischaemic secondary injury and that glutamate is either a marker for or an agent of ischaemic secondary injury. We examine time course of CSF glutamate release in two groups of TBI patients, with and without an early ischaemic insult identified by discriminate analysis based on an elevated arteriovenous oxygen difference $\left(\mathrm{AVDO}_{2}\right)$ within 24 hours of injury associated with electrophysiological deterioration as measured by quantitative somatosensory evoked potentials (SSEPs).

Methods: From July 1987 to November 1995, intensive monitoring of 55 patients with severe TBI (GCS $\leq 8$ after resuscitation) included twice daily $\mathrm{AVDO}_{2}$ and CSF glutamate levels and hourly SSEPs for an average of five days. SSEPs were quantitated as summed peak to peak amplitudes.
Results: Patients with early ischaemia did not differ in their initial SSEPs from the nonischaemic group but suffered a deterioration in their SSEPs to levels significantly below those of the nonischaemic group by day five post injury. At no time following injury could a significant difference in CSF glutamate levels be demonstrated between the two groups.

Conclusions: Although CSF glutamate levels are elevated after TBI, CSF glutamate does not appear to be a marker for or an agent of ischaemic secondary injury. Glutamate excitotocity may be an independent mechanism of secondary injury which we have not yet dissected from other causes of ongoing damage after TBI.

\section{P-021}

Neurosurgery in the Elderly: Retrospective Analysis of 71 Patients

F. Maroun, H. Reddy, J.C. Jacob, J. White, G. Murray (St. John's, Newfoundland)

This study was conducted to ascertain the effectiveness of neurosurgery in elderly patients. Previous studies have disagreed as to prognosis for various neurosurgical procedures for geriatric patients. Of 136 admissions of patients over 65 in the one year time interval, there were 114 patients of mean age 71 . Of the 71 surgical patients, there were $36(50 \%)$ with spinal surgery, 11 (15\%) for evacuation of hematomas, $4(6 \%)$ for aneurysms, 20 $(28 \%)$ for tumours and $1(1 \%)$ for neurectomy. Overall, 60 of the 72 surgical procedures had favourable outcomes with $83 \%$ success rate. The detailed result of this study shows that neurosurgery can be done successfully in patients with advanced age and any increase in years of good quality of life can certainly be appreciated at all ages.

\section{P-022}

\section{Surgical Anatomy, Imaging and Results of Treatment of the} Chiari Malformation in Adults

\section{C.H. TATOR (Toronto, Ontario)}

The author has reviewed the surgical anatomy, imaging and results of treatment of 47 adult patients with Chiari malformation 20 of whom have had posterior fossa decompression as the primary treatment. In every posterior fossa case, the locations of the brainstem and fourth ventricle as determined by imaging and surgery were compared. At surgery, the locations of the cerebellar tonsils, the obex and the lowest segment of the fourth ventricle (identified by its ependymal lining) were determined in relationship to the foramen magnum. In all cases, the obex and the lowest point of the fourth ventricle were found to be located at or below the level of the foramen magnum, proving that the tonsils and the brainstem were herniated through the foramen magnum in every case. Thus, at surgery all cases met the criteria of the Chiari II malformation. In contrast, the obex and lowest portion of the fourth ventricle were frequently incorrectly located on the basis of imaging. The results suggest that the Chiari malformations in adults encompass a spectrum of cases all with herniation of the brainstem of varying extent through the foramen 
magnum, and with a variety of associated lesions such as hydrocephalus, syringomyelia and myelodysplasia. The study also showed that decompression of the brainstem for symptomatic Chiari malformation in adults is an excellent treatment with amelioration of symptoms in almost all cases and with minimal morbidity and no mortality. In cases with associated syringomyelia, posterior fossa decompression was also usually effective for treatment of the syringomyelia.

\section{P-023}

\section{Spontaneous Intracranial Hypotension}

\section{MaharaJ, R.O. Holness (Halifax, Nova Scotia)}

Spontaneous intracranial hypotension is a rare but distinct clinical entity with about 50 reported cases. We report 2 cases illustrating investigations and treatment options.

Case I: Female, 37 years, experienced abrupt onset of aching headache, relieved in minutes by lying flat and exacerbated by head elevation. Nausea, vomiting, tinnitus and interscapular pain were present. Physical exam was normal. Cerebrospinal fluid (CSF) pressure was $50 \mathrm{mg} \mathrm{Hg}$ and, on repeat, and a "dry tap" resulted. Radionucleotide cisternography and MRI were normal. CT myelogram demonstrated contrast leakage at T9-10. An initial blood patch failed, but when repeated at 10 days, resulted in complete headache resolution which has been maintained.

Case II: A 23-year-old male with rare migraine headaches presented with daily headaches, unresponsive to conventional drugs. Nausea, vomiting and neck pain were present. MRI demonstrated a narrowed CSF space with perithecal cervical swelling. CT/myelography demonstrated leakage at the C2 nerve root sleeve on the left. A $1 \mathrm{~cm}$ fistula was demonstrated and repaired. His symptoms gradually resolved over a few weeks.

CT myelography was the definitive investigation in both cases, who remained headache free for 1 and 3 years respectively. Although some cases resolve spontaneously, prompt diagnosis and treatment in our cases resulted in an excellent outcome.

\section{P-024}

The Surgical Management of Axillary Nerve Injuries: Experience of the Toronto Hospital Peripheral Nerve Clinic

\section{R. Saunders, J. Su, A.Guha (Toronto, Ontario) B.Graham, (Calgary, Alberta)}

The authors have reviewed 18 patients with injuries of the axillary nerve associated with clear deltoid weakness and who were treated with a surgical procedure. The study sample was from a typical trauma population which was predominantly male (83\%) and relatively young (avg. age 33 years). Approximately half of the patients $(45 \%)$ were injured as a result of a motor vehicle accident, while the remaining sustained injuries in sports-related accidents, a fall from a height or a gun shot wound. The axillary nerve was injured in isolation in 15 cases.
Clinical examination and electrodiagnostic testing with a failure to show spontaneous recovery was the determining factor for surgery. Nine patients were treated within six months and the remaining nine patients were operated upon after this time. The reason for delayed surgery was invariably that the patients were referred for our opinion after a prolonged period of clinical denervation. One-third of patients were treated by an external neurolysis and the remaining twelve patients required excision of a neuroma-in-continuity and sural nerve grafting. The decision to perform a neurolysis or a nerve grafting was based on the results of intraoperative stimulation of the axillary nerve.

The outcome of treatment was established by examining function of the axillary nerve using standard impairment measures such as the recovery of motor power and skin sensation and also by administering a health related quality of life questionnaire in an attempt to determine the patient-perceived extent of recovery. The results of treatment in the group of patients treated within six months of their injury was contrasted to that of patients treated later than six months after the onset of paralysis.

\section{P.025}

Occipitocervical Fusion with the Cotrel-Dubousset plate: Longterm Results in 25 Patients

D. Louw, M. FeHLINGS (Toronto, Ontario)

Background: Occipitocervical fusion techniques for instability of the occipitocervical articulation are both varied and potentially challenging. We present our experience in a prospective series of 25 cases, the majority of which were performed with the Cotrel-Dubousset (CD) horseshoe plate.

Methods: Axial and occipitocervical instability was confirmed by plain radiographs, CT and MRI. The CD plate is usually secured to the skull with $12 \mathrm{~mm}$ long, $3.5 \mathrm{~mm}$ diameter screws. Burr holes and cable fixation are employed in cases with deficient occipital bone. The rods $(5 \mathrm{~mm}$ diameter, 60 or $80 \mathrm{~mm}$ length) are fastened to the spine with sublaminar Danek cables. Supplemental autogenous bone graft was used in all cases.

Results: The average age of our patients was 59 years with a range of 17 to 82 . Mean follow-up of surviving patients was 24 months (range 11-43) and the average number of levels fused 6 (range 0- C3-0-T1). Three deaths occurred within the first 3 months of surgery, 2 from progression of malignant disease. In long term follow-up, 1 patient died of glioblastoma and another of complications of rheumatoid arthritis.

Conclusions: Solid occipitocervical arthrodesis is readily accomplished with the $\mathrm{CD}$ horseshoe plate. It is proven to be a safe, versatile technique and affords advantages over some alternative methods.

\section{P-026}

Occipital Condyle Fractures: Report Of 3 Cases And Review Of The 96 Reported Cases

\author{
S. Tul, C.H. Tator (Toronto, Ontario)
}


Background: Occipital condyle fractures (OCF), a unique and rare entity was first described on the basis of postmortem examination by Bell in 87 and since then only sporadic case reports have appeared. The present study reviews the 58 living and 38 postmortem cases which have been reported in 40 articles. Three recent cases of OCF in our Centre prompted a review of the incidence, clinical presentation, diagnosis and treatment of this entity. Previous reports have suggested that the triad of neck pain, lower cranial nerve deficit and normal cervical spine $\mathrm{x}$-rays indicate the diagnosis of OCF.

Methods: A retrospective evaluation was performed on 93 consecutive trauma victims presenting to the Toronto Hospital (Western Division) from January 1993 to February 1994, who had CT scans available for analysis of OCF. The medical records, plain $\mathrm{x}$-rays, and $\mathrm{CT}$ were evaluated for the presence of an OCF.

Results: The review of previous reports showed that cranial nerve deficits were found in $3 \%$, of which $38 \%$ were delayed. Only three published cases of OCF with neck pain but without cranial nerve deficits have been described to date. The cervical spine $x$-rays were normal in $96 \%$ of the cases. In our retrospective review, only one of 93 cases had an asymptomatic OCF on CT.

Conclusion: OCF is a diagnostic challenge. Conservative treatment has been most commonly used, although there is uncertainty about the stability of the different fracture types and their management.

\section{P-027}

\section{A Juxta-Facet Cyst of the Lumbar Spine Causing Recurrent Neurogenic Claudication After Treatment for Spinal Stenosis - Case Report}

\section{HODAIE, F.J. EsPINOSA, J.P. Rossiter (Kingston, Ontario)}

Juxta-facet cysts for the lumbar spine can be included amongst the uncommon causes of radiculopathy, and less frequently lead to symptoms of neurogenic claudication. These cysts generally develop slowly in the context of degenerative lumbar spine disease, commonly at the L4-5 level, a segment with relative increased mobility. We present the case of a patient with grade I spondylolisthesis at the L3-4 level with symptoms of neurogenic claudication, who was treated with lumbar spinal fusion and recovered with no neurological deficit. Subsequent to a fall 12 months after surgery, she presented with recurrent disabling symptoms of neurogenic claudication. Magnetic resonance imaging (MRI) revealed severe compromise of the thecal sac posteriorly by a large heterogeneous cystic mass at the L2-3 level. Operative removal of the mass and pathological examination identified a juxta-facet cyst, with evidence of hemorrhage within it.

This case illustrates the rapid development of recurrent symptoms of neurogenic claudication, due to a juxta-facet cyst at the L2-3 level. Redistribution of pressure forces after the lumbar fusion resulted in the formation of an area of increased relative hypermobility at the L2-3 level, which subsequent to joint expansion after trauma likely caused the formation of a juxtafacet cyst.

\section{P-028}

\section{Thoracic Disc Herniation Causing Abdominal Hernia}

R. Brownlee, J. Krcek (Calgary, Alberta), G. MoHAMMADZADEH (Winnipeg, Manitoba)

Background: Abdominal hernias due to focal muscle weakness are uncommon. It has been reported in diabetes, herpes zoster, lyme disease, syringomyelia and lumbar disc herniation. There have been no previous reports of a ventral hernia caused by a thoracic disc herniation.

Methods: A 48 year old male experienced the onset of leftsided back pain the day after shoveling snow. The pain increased in intensity over the subsequent 3 weeks, remaining well localized with no radiation. It was aggravated by back movements and coughing. Approximately 10 days after the onset of pain, the patient noted a painless swelling in his left upper abdomen which was present when standing, increased with coughing and disappeared when lying flat. Examination revealed decreased touch and pin prick sensation from the left costal margin to the iliac crest extending from the midline anteriorly to the midline posteriorly. There was no palpable mass and no abdominal tenderness. An abdominal ultrasound and computed tomographic (CT) scan were normal. Magnetic resonance imaging of the spine revealed an extradural mass centered at the T10-11 disc space with extension into the left neuroforamen. Posterolateral decompression of the left $\mathrm{T} 10$ and $\mathrm{T} 11$ roots with removal of the mass was accomplished. Pathologic examination showed the mass to consist of degenerative disc material with no evidence of infection or neoplasia.

Results: The patient's pain was completely gone immediately after surgery and he was discharged from hospital the following day. A subjective improvement in touch and pin prick sensation were noted at that time but the muscle weakness persisted.

Conclusions: Radiculopathy secondary to thoracic disc herniation is an unusual cause of abdominal muscle weakness presenting as a ventral hernia.

\section{P-029}

Spinal Epidural Lipomatosis with $90^{\circ}$ Rotation of the Underlying Spinal Cord: A Case Report

\author{
S. Tuli, J. Hurlbert, D. Mikulis, R. Fleming (Toronto, \\ Ontario)
}

Spinal epidural lipomatosis most commonly occurs in patients with morbid obesity, prolonged high dose corticosteroid use or Cushings syndrome, although sometimes it is idiopathic. It may or may not be associated with clinical evidence of myelopathy. Rotation of the spinal cord underlying an area of lipomatosis has not previously been reported. Rotation of the spinal cord in the absence of overlying lipomatosis has been reported in one published case report. We present a 44 year old male with a four year history of progressive spastic weakness of his legs, and lipomatosis behind the thoracic cord, whose nerve roots were found to exit from the posterior and anterior midline planes of the dura, suggesting a $90^{\circ}$ rotation of the thoracic 
cord. MR scans clearly demonstrated the segmental thoracic nerve roots exiting from the posterior midline of the dura, a finding confirmed at surgery. After removing the vertebral arches, the lipomatous tissue was partially resected and two nerve roots exiting from the back of the dura were divided. There was gradual improvement in the myelopathic symptoms postoperatively.

MR scan 6 months after surgery revealed minimal residual lipomatous tissue and absence of cord rotation, the nerve roots exiting from their appropriate right and left locations. The lipomatous tissue may have been secondary to premature or incomplete dysjunction with asymmetric retention of mesenchymal tissue, rotating the cord. Alternatively, the lipomatosis might have been an epiphenomenon and cord rotation an isolated congenital anomaly. Direct mechanical stress due to deformation of the rotated cord, rather than compression, is postulated as the mechanism of the myelopathy.

\section{MULTIPLE SCLEROSIS AND NEUROVIROLOGY}

\section{P-030}

\section{Which Test of Upper Limb Function Should Be Used in} Multiple Sclerosis (MS) Treatment Trials?

J.H. Noseworthy, M. Rodriguez, B.G. Weinshenker, K.A. Stolp-Smith, J.A. Weis, P.C. O'Brien, J.A. Ferguson, T. Petterson (Rochester, Minnesota)

Objective: To determine the performance characteristics of tests of upper-limb function being used in MS treatment trials.

Background: There is little data on test performance (reliability, sensitivity, specificity, the influence of hand dominance, etc.) for the box and blocks (BBT) and 9 hole peg test (9HPT).

Design/Methods: In a placebo-controlled, double-blind trial of IVIg (target-76 pts), tests of motor function (clinical exam, BBT, 9HPT, isometric measures, videotapes) are repeated every 3 months to detect improvement. Clinically normal upper limbs are called "normal" for this study.

Results: 38 patients have been studied. "Normal" MS upper limbs are slightly impaired on these tests compared with literature controls. BBT is less sensitive to the influence of limb dominance. Internal consistency is high with both tests. Both tests show a mild practice effect within the same visit. 9HPT performance remained nearly constant in clinically stable, "normal" limbs between the screening and enrollment visits. Using literature controls to normalize test values, the sensitivity of the two tests is virtually equal ( $>2 \mathrm{SD}=$ positive test): dominant hand 78\% BBT, 78\% 9HPT; nondominant-73\% BBT, 77\% 9HPT. BBT has slightly higher specificity: dominant-63\% BBT, $50 \%$ 9HPT; nondominant 67\% BBT, 50\% 9HPT.

Conclusions: There are currently insufficient differences in the performance of these tests of upper limb function to advise which is preferable for use in a clinical trial. The relative responsiveness of these tests to treatment effects will await the primary analysis of the trial (12/96; supported by NIHNS31506).
P-031

Visual Evoked Potentials Correlate with Disability Score in Multiple Sclerosis (MS)

P. O'Connor, C. TANSEY (Toronto, Ontario)

Background: Multi modal evoked potentials (EP) may measure burden of disease in MS. We examined how well certain EP measures correlated with disability and disease duration in an MS clinical trial.

Design \& Methods: Baseline multi modal EPs (visual, auditory, four-limb somatosensory and upper-limb central motor) were performed on 60 patients with relapsing-remitting MS enrolled in a trial of Oral Myelin (Myloral) therapy. Patients were between ages 18-45 with a minimum of 2 attacks in the previous 2 years and an extended disability status score (EDSS) of between 0 -4.5. Mean P100 latency (P100), lower limb somatosensory central conduction time (LSEP), and upper limb central motor conduction time (MEP) were correlated against EDSS and disease duration.

Results: The P100 correlated better with the EDSS $(r[$ Pearson correlation coefficient $]=.64$, with $p=.0001$ ) than did LSEP and MEP ( $\mathrm{r}=.44$ and $.30, \mathrm{p}=.001$ and .03 respectively). The correlation of P100 with disease duration was mild $(r=.25$, $\mathrm{p}=.07$ ), as was the correlation of disease duration with EDSS $(\mathrm{r}=.31, \mathrm{p}=.03)$.

Conclusions: Mean P100 latency of the visual EPs correlates much better with EDSS in MS than do reported brain MRI measures such as lesion area( $r \cong .22)$,or somatosensory and central motor times.

\section{P-032}

\section{Survival of Multiple Sclerosis in London-Middlesex County} Ontario

W. Hader, M. Hader (Saskatoon,Saskatchewan), G. Ebers (London, Ontario)

The disability and survival of Multiple Sclerosis patients in a population-based cohort prevalent group I January 1984 was reviewed. Of the original 283 clinically definite cases, the current status was ascertained in $96.3 \%$ (270). In this group $209(74.6 \%)$ are alive, $61(21.7 \%)$ deceased, $10(3.6)$ unable to trace and 3 considered as non-ms. The mean age of onset for females was $28.8 \pm 9.7$ years, range $10-58$ years, and $31.2 \pm 10.9$ years for males, range 6-66 years.

The mean age of 155 females is now $52.7 \pm 12$ years, and $56.5 \pm 12.7$ years for the 54 males. The mean duration of disease is $24.9 \pm 9.9$ years for females, and $25.5 \pm 8.0$ years for the males. The mean age of death of 36 females is $61.2 \pm 13$ years, and $61.0 \pm 11.7$ years for the males.

The outcome according to Kurtzke Disability Status Scale was compared for 1984 and 1995. Minor disability DSS $<2.5$ was $38.2 \%$ and $20.4 \%$, mild DSS $3-5.5$, was $14.3 \%$ and $10.1 \%$, moderate DSS $6-7.5$, was $24.7 \%$ and $20.0 \%$, and severe DSS 8 9 was $22.8 \%$ and $27.1 \%$.

The median survival after onset is 43 years. The estimated 
survival for 25 years is $82 \%$, and $67 \%$ for 35 years, with no significant difference between the sexes. Survival was better than previously reported and probably reflects the quality of nursing care in local long-term institutions.

\section{P-033}

Acute Transverse Myelitis Linked to Multiple Sclerosis. A Case Report of Familial Multiple Sclerosis and Transverse Myelitis

\section{H. WILSON, (Scarborough, Ontario)}

Background: Acute transverse myelitis (ATM) is rarely the first sign of multiple sclerosis (M.S.). The association between ATM and M.S. is more common in Orientals than Caucasians.

Method: A report of a family in which a Caucasian mother developed M.S. at age 26 and a son developed M.S. at 23 years. The CSF and histocompatibility complex were studied in all members of the family. All members had MRI of the brain. The mother developed M.S. 20 years after contracting ATM.

Results: The mother was DRB4 positive. The son with M.S. and an asymptomatic son were DRB3 positive and DRB4 positive.

Conclusion: It is concluded that there is a significant link between the pathogenesis of ATM and M.S. This will be discussed. The clinical picture of the mother will be analyzed to determine if there were features of her type of ATM that would predict the development of M.S.

\section{P-034}

Intense Immunosuppressive Therapy for Progressive Multiple Sclerosis (MS): Experience Using a Booster Regimen of Cyclophosphamide and Methylprednisolone

M.S. Freedman, R.F. Nelson, H. Rabinovitch, S. Christie, L. Denis, C. Corman, M. Benavente (Ottawa, Ontario)

Background: There is to date no recognized or proven therapy for progressive MS. Generalized immunosuppression has been shown to have a modest effect on slowing progression in MS but has been unfavourable due to unacceptable toxicity. We have used a less toxic protocol of "pulse" intravenous methylprednisolone and cyclophosphamide, first described by Hohol et al (Ann Neurol 32:256, 1992), to treat our patients over the past 2 years and describe our experience.

Methods: Patients with clinically definite and progressive MS were selected on the basis of a continued deterioration over 2 consecutive 6 month periods bordering on the loss of ambulation. Details of the treatment regimen will be presented. Briefly, patients were induced in hospital and maintained on monthly boosters for the first year and 6 weekly boosters for the second year. Patients were steadily monitored for toxicity and clinically assessed every 3 months. In some cases baseline MRI and yearly scans were performed and in the last six months a Quality of Life questionnaire was added.

Results: A total of 17 patients have been treated thus far over periods of 2-24 months. More updated results will be presented, however at this time 8 patients demonstrated a significant improvement, as determined by at least a decrease of 0.5 on the Expanded Disability Status Score (EDSS). Two patients continued to deteriorate and 4 remained stable. On 3 patients, follow-up status is pending. Four patients discontinued treatment, but none due to any significant toxicity. Of these, 1 started to deteriorate within 6 months of stopping, 2 improved and remained stable and the other had deteriorated prior to discontinuing therapy. No significant toxicities were encountered. Individual details will be presented.

Conclusions: We describe our experience with a relatively well tolerated regimen of steroids and cyclophosphamide for the treatment of MS patients with imminent progression. 12/17 of our treated patients stabilized or improved thus far suggesting this regimen may be beneficial to a subset of MS patients.

\section{P-035}

\section{Atypical Clinical Manifestations of HTLV-1 Infection}

\section{A.G. Douen, C.E. Pringle, A. Guberman, (Ottawa, Ontario)}

Background: The Human T lymphotropic virus (HTLV) type 1 is associated with a chronic progressive myelopathy termed Tropical Spastic Paraparesis (TSP) and/or HTLV-1 Associated Myelopathy (HAM). Characteristically patients with HAM/TSP have spasticity and/or hyperreflexia in the lower extremities, urinary bladder disturbance, and lower extremity muscle weakness. However, a few cases of isolated polymyositis in HTLV-1 infected patients in Jamaica have been reported.

Methods: We report a 67 year old woman from Trinidad, with chronic progressive quadriparesis, muscle wasting in distal extremities, moderate distal upper extremity weakness (4/5) in wrist and finger extensors and flexors, apart from the left wrist flexors, which were $0 / 5$ and bilateral foot drop. There were no sensory changes and she was areflexic. Gait was wide based and ataxic with a steppage component bilaterally. Higher functions including memory were intact.

Results: Serum CK was 1091 U/L. EMG and NCS were consistent with both an inflammatory myopathy and an axonal neuropathy. Muscle biopsy confirmed a myositis. An MRI performed in 1989, at the beginning of her illness, showed multiple bilateral confluent and focal subcortical white matter lesions. A repeat MRI in 1995 showed little interval change. Serum antibodies to HTLV-1 were identified. Treatment with oral steroids resulted in marked improvement in strength, and decreased CK; $677 \mu / \mathrm{L}$.

Conclusions: This case represents an unusual presentation of steroid responsive polymyositis and peripheral neuropathy in the context of radiological evidence of cerebral white matter involvement, in association with HTLV-1.

\section{P-036}

\section{Primary Headaches in Patients with HIV-Infection}

S. Mirsattari, C. Power, A. NATH, (Winnipeg, Manitoba)

We retrospectively studied the clinical characteristics of prima- 
ry headaches occurring in Human Immunodeficiency Virus (HIV1)-infected patients. Of a total of 75 patients seen in Neuro-AIDS clinic from 1990 to 1995,10 patients were diagnosed with primary headaches. All other causes of headaches were excluded by CSF examinations; serologic studies and neuroimaging. Seven patients had migraine headaches (4 patients with common migraine, 1 with a variant of common migraine, 1 with classic migraine, and 1 with status migrainous). Two patients were diagnosed with tension-type headaches, and 1 with cluster headache. Six patients had a past history and/or a family history of headaches. Mean CD4 cell count was 261 cells $/ \mathrm{mm}^{3}$ (range $17-656$ cells $/ \mathrm{mm}^{3}$ ). Four patients were on antiretroviral drugs at the time of the onset of headaches. Six patients with vascular headaches were followed for a mean of 15 months (range 6-36 months). Five of these patients developed intractable headaches. We conclude that, i) primary headaches are not uncommon in patients with HIV infection, ii) they may present for the first time in patients with severe immunesuppression, iii) no relationship to antiretroviral drugs was found, and finally, iv) these headaches frequently do not respond to conventional management and carry a poor prognosis.

\section{P-037}

\section{Devic's Neuromyelitis Optica in a Patient with AIDS}

\section{T.G. Colllier AND M. Jones (Vancouver, British Columbia)}

Background: Devic's Neuromyelitis Optica (NMO) is a syndrome of acute optic neuritis (often bilateral) with transverse myelitis. NMO is classified along with demyelinating diseases such as Multiple Sclerosis (MS), but in its pure form the head MRI fails to show multiple areas of white matter abnormality and the patients do not develop neurologic dysfunction in other areas of the nervous system. Although MS has been described rarely in HIV infection, NMO has never been reported.

Methods: We describe a 31-year-old HIV-positive man with a six month history of AIDS defined by CD $4<30$ who presented to hospital with tonic-clonic seizures, fever, and encephalopathy. Three days later, as his mental status was clearing, he developed a myelopathy with a T-2 sensory/motor level and bilateral visual loss. His deficits were complete 48 hours later and eight months after onset he remains blind and paraplegic. The rest of his neurologic exam remains normal.

Results: MRI of his head was unremarkable, while MRI of his spine showed subtle increased signal in the cord at C7- T2. Two CSF examinations failed to identify any infectious agent, and oligoclonal banding was absent. PCR for Herpes Simplex Virus and Cytomegalovirus on CSF were negative. Dexamethasone $16 \mathrm{mg} /$ day started on hospital day 4 and an induction course of ganciclovir, started on hospital day 12 , had no effect on his deficits.

Conclusions: Autoimmune demyelinating illnesses are exceedingly uncommon in HIV infected individuals. However, rare cases of MS have been described, and we have presented a case that satisfies the current clinical description of NMO.
P-038

Fatal Familial Insomnia: A Chinese Kindred with the 178 Aspargine Prion Protein Gene Mutation and 129 Methionine Homozygosity

H. Feldman, G.J. Green, B.C. McGillivray, K. Berry, (Vancouver, B.C.) R.B. Petersen, P. Gambettr, (Cleveland, Ohio).

Fatal familial insomnia (FFI) is a rare prion disease with clinical features that include dementia, dysautonomia, progressive sleep disorder, and motor dysfunction. FFI has been linked to the 178aspargine (178 Asn $) 129$ methionine (129Met) haplotype of the prion protein gene (PRNP). Previously reported families have been restricted to European ancestry.

We report on the clinical, genetic, and neuropathologic features of several members of two generations of a Chinese kindred with FFI. Neuropathology has shown characteristic selective anterior and medial thalamic nuclear degeneration with cerebellar olivary nuclear loss. The genotyping of an affected male demonstrates a $178^{\mathrm{Asn}}$ codon mutation of the PRNP with met homozygosity at codon 129. On MR spectroscopy his cortical $\mathrm{N}$-acetylaspartate/creatine ratio has been progressively declining through his course.

This is the first reported Oriental kindred with FFI. With improved phenotypic recognition of FFI and with the potential to genotype the PRNP it is anticipated that there will be improved recognition of affected individuals and families. Both the genotype of $178^{\mathrm{Asn}}, 129^{\mathrm{Met}}$ and the phenotypic expression in this Chinese family are virtually the same as the reported families of European ancestry. The early onset and rapidly declining clinical course are consistent with the prior observations of homozygosity of $129^{\mathrm{Met}}$ in FFI.

\section{PAEDIATRIC NEUROLOGY - GENERAL}

\section{P-039}

The Connatal Form of Pelizaeus-Merzbacher Disease May Be Pyridoxine-Responsive with Reversal of White Matter Changes on MRI

E.C. Wirrell, P.R. Camfield, M.D. Bethune, M.D. Riding, (Halifax, Nova Scotia)

Case Description: A male infant presented at three months of age with episodic large amplitude, pendular nystagmus, seizures and psychomotor delay. His EEG showed small amounts of multifocal sharp waves originating from both hemispheres but no electrographic changes were noted with the episodes of nystagmus. His MRI showed severe lack of myelination. He was clinically diagnosed with the connatal form of PelizaeusMerzbacher disease, but subsequently proved to be pyridoxineresponsive, with resolution of his nystagmus and seizures, catchup of developmental milestones and significant improvement in the white matter on MRI.

Conclusions: The connatal form of Pelizaeus-Merzbacher disease may be pyridoxine-responsive. An adequate trial of pyri- 
doxine should be given to any child presenting with this disorder. White matter changes initially noted on imaging studies may be reversible and marked developmental gains may be seen.

\section{P-040}

\section{Atypical Rett Syndrome in Triplets}

\section{P. Lidman, D.B. SinClaiR (Edmonton, Alberta)}

Rett Syndrome is a common neuro-developmental disorder that manifests almost exclusively in females. Classically, Rett Syndrome requires normal early development with gradual loss of purposeful hand skills, psychomotor regression, acquired microcephaly, appearance of stereotypic hand movements and gait apraxia. As awareness of this syndrome increases, there is a rising number of cases with "Rett-like" clinical manifestations that do not fulfill the usual criteria for Rett Syndrome. We report a set of monozygotic triplet girls with Rett-like features. All 3 children were diagnosed with cerebral palsy, and the diagnosis of Rett Syndrome was not considered until the second born girl began to lose the ability to use her hands and developed hand wringing and episodic hyperventilation at 7 years of age. The third born girl had some autistic features such as head banging, rocking and also hand-mouthing. The first born girl, although the most severely delayed, does not presently exhibit signs of Rett Syndrome.

These triplet girls support a genetic etiology (X-linked dominant mutation) and are the first reported cases of triplets with Rett Syndrome. Although the girls are monozygotic, they show discordant atypical features of Rett Syndrome with variable rates of disease expression.

Rett Syndrome may present with atypical features and may have variable rates of disease progression even in affected siblings. Rett Syndrome should be considered in females with atypical Cerebral Palsy even if strict Rett criteria is not met.

\section{P-041}

\section{Troublesome Sensitivity to Head Movement: A Familial Vertiginous Disorder}

K. Gordon, P. Camfield, E. Wirrell, K. Clarke (Halifax, Nova Scotia)

Case Description: An 18 month infant presented with a three week history of forced deviation of the head to the right when laid supine for diaper changing. She would scream such that her parents had resorted to changing her while suspended vertically. Since walking, she intermittently appeared to be off balance after turning. She had never tolerated swinging.

Family History: Both the child's mother and maternal grandmother have difficulty with sudden turns. After having gone around a corner while driving, mother has to pull over to the side of the road as "I don't know where I am".

Examination: The child screamed when moved to the supine position, and her head deviated to the right. There was no nystagmus. She avoided any swinging or turning. Her mother complained of marked dizziness with the Hallpike maneuver, and had exaggerated nystagmus with her head deviated to the left. Both mother and grandmother were able to pirouette once while walking before becoming extremely dizzy and grasping for the wall.

Investigations: The child had a normal CT scan. Mother's ENG was borderline abnormal.

Conclusion: This family has an apparent autosomal dominant hypersensitivity to vertigo.

\section{P-042}

\section{Hypoxic Seizures In Children With Breath-holding Spells}

\section{R. MunN (Toronto, Ontario)}

Breath-holding spells (BHS) are common in childhood and are occasionally followed by hypoxic seizures. The purpose of this study was to identify factors that might predict which children with BHS have associated hypoxic seizures.

All children referred for BHS between Jan. 1994 and Dec. 1995 ( $n=32$, male=15) were retrospectively reviewed. Twentyone had cyanotic spells, 8 pallid and 3 had both types of spell. Nine of the children had at least one tonic-clonic seizure during a BHS (seizure group). The seizure group was compared to the remaining 23 children (controls). All children in the seizure group had cyanotic BHS.

The seizure group had more frequent BHS (all $>1 /$ month) compared to the controls $(12>1 /$ month). The seizure group had a mean onset of BHS at 8.5 months of age compared to 13.5 months for the controls. Three children in the seizure group had an increase in BHS during a concurrent infection which appeared to provoke a seizure.

In conclusion, children with BHS and seizures in this study all had cyanotic spells, earlier onset of BHS and more frequent spells when compared to controls.

\section{P-043}

Progressive Encephalopathy with Edema, Hypsarrhythmia and Optic Atrophy (PEHO) Syndrome: A North American First!

\section{Shevell, P. Colangelo, E. Tracey and B. Rosenblatt} (Montreal, Quebec)

Background: Progressive encephalopathy with edema, hypsarrthymia and optic atrophy (PEHO) syndrome was first reported in 1991 and thus far has only been reported in the Finnish population with the exception of two Japanese siblings. Essential features for diagnosis include; the onset of seizures between 2-52 weeks of age, eventual infantile spasms with hypsarrhythmia, early severe arrest in psychomotor development, profound hypotonia and absent visual fixation. Additional features present in most patients include subcutaneous edema, microcephaly, minor dysmorphic features, and optic atrophy. Evolving progressive cerebellar atrophy is the characteristic neuroradiologic finding.

Methods: A patient with all the essential features of PEHO syndrome was diagnosed. 
Results: A French-Canadian child first presented at 3 months with difficult to control minor motor seizures that evolved into infantile spasms with a hypsarrthymic EEG. Profound global psychomotor regression and visual impairment ensued. Examination revealed microcephaly, minor dysmorphic features, optic atrophy, severe hypotonia and hyperreflexia. Extensive biochemical and metabolic investigations including a nerve/muscle/skin biopsy were negative. MRI studies documented an evolving cerebellar atrophy at 18 months of age.

Conclusion: This represents the first reported observation of PEHO syndrome in the North American population. This extends the genetic reach of this syndrome and validates its authencity.

\section{P-044}

Influenza A Encephalitis involving Basal Ganglia in a TwoYear Old Girl - A Case Report with a Review of the Literature

\section{R. SMITH, I. TEIN (Toronto, Ontario)}

Background: Influenza encephalitis has been associated with basal ganglia involvement, although recent literature is scant.

Methods: We present a case report of a 2-year old girl with Parkinsonism secondary to Influenza A encephalitis.

Results: One week following onset of an upper respiratory infection, a previously healthy two year old girl presented with four days of progressive loss of purposeful limb movement, facial expression and hypophonia. There was no history of altered conciousness, seizures or sphincter disturbance. There had been no prior episodes, and no history of trauma, ingestion or vaccination. On presentation, she was afebrile. She could fix and follow and cried if upset. She had a paucity of facial expression but normal corneal responses and a brisk jaw jerk and some pooling of secretions despite a good gag reflex. There was limited spontaneous limb movement and she had a low frequency tremor of all limbs. She had signs of a spastic quadriparesis with subtle superimposed rigidity. Magnetic resonance imaging demonstrated increased signal in the caudate, putamen and globus pallidus symmetric bilaterally and in the floor of the fourth ventricle. CSF revealed a monocytosis with normal sugar and protein. PCR for Herpes, EBV and CMV were negative. The serum complement fixation titre for Mycoplasma pneumoniae was insignificant. Influenza $A$ antigen was detected in nasal swabs by ISA. Serum and CSF titres for other encephalitides were negative. Serum and CSF lactate were normal. EEG demonstrated frontal intermittent rhythmic delta activity and diffuse slowing. Over two weeks she developed progressive severe rigidity and tremor and exhibited one episode of oculogyric crisis. She required gavage feeding. Treatment with Amantadine and Trihexyphenidyl showed no immediate benefit. By four weeks, she had regained some useful limb function. The early recovery in our child is encouraging. A trial of Levodopa has been recently initiated. We present her long-term follow up (with videotape) and a literature review.

Conclusion: Influenza A encephalitis should be considered in the differential of post-encephalitic Parkinsonism.
P-045

\section{Multimodal Evoked Potentials in Childhood Neurofibromatosis}

B.L. Banwell, M.J. TaYlor, W.J. Logan, S. Blaser, (Toronto, Omtario)

Neurofibromatosis type 1 (NF1) presents in childhood with central and peripheral nervous system lesions. Evaluation includes close clinical monitoring and neuroimaging. Multimodal evoked potentials provide information on brainstem, visual cortical and dorsal column pathway integrity. Little has been published on the use of EP studies in patients with NF.

The records of 30 children with NF1 evaluated in the evoked potential lab at The Hospital for Sick Children were reviewed. Brainstem auditory evoked potentials (BAEP), visual evoked potentials (VEP) and upper limb somatosensory evoked potentials (SEP) were reviewed in a blinded fashion by one examiner (MT). Ten children had follow-up examinations $\mid$ to 6 years after initial assessment.

Significant abnormalities in one or more modalities were seen in 21 of 30 children: BAEP, 16/27 abnormal; VEP, 17/27 normal; SEP, $18 / 24$ abnormal. In the 10 children with repeat studies, abnormalities seen on first assessment remained or became more marked in 7 . One child had normal results on both studies, one child had the BAEP return to normal, but developed abnormal VEP, and one child had only BAEP done initially (abnormal) and VEP done on second assessment (abnormal).

Multimodal evoked potentials are frequently abnormal in children with NF1 and remain so over time. It is possible that these abnormalities reflect areas of dysplasia or they may be markers of future schwannomas, acoustic neuromas or optic nerve gliomas. Clinical and neuroradiological correlation in these 30 children is currently underway.

\section{P-046}

CACN-L: A Discussion Group for Canada's Pediatric Neurologists on the Internet

MEMBERSHIP OF THE CACN-L, (CACN-L@AC.DAL.CA).

Background: The CACN identified communication as an issue for its membership during the 1994 CCNS.

Introduction: In July 1995, an e-mail discussion group was established through the mailserver at Dalhousie University. Membership is restricted to current members of the CACN. Initially 10 members were recruited, however membership has doubled to 21 , perhaps related to increasing interest in the Internet. To date, postings have been limited.

Methods: A questionnaire concerning the $\mathrm{CACN}-\mathrm{L}$ was sent to 21 members 96.01 .02 .

Results: 5 questionnaires were not delivered ( 3 mail addresses were incorrect and for 2, the mailserver at the HSC was down). Of the 16 delivered surveys, $12(75 \%)$ were returned within one week, $6 / 12$ within 24 hours. 50\% of respondents had been using e-mail for over 1 year, primarily for professional reasons. For two thirds, the CACN-L was the only discussion group 
that users were a part of. Over the next year, participants projected that $57 \%$ ( $\pm 21 \mathrm{SD}$ ) of postings would be clinical, $26 \%$ ( \pm $14 \mathrm{SD}$ ) would be research oriented, and $16 \%$ ( $\pm 14 \mathrm{SD}$ ) would be related to administrative issues of the CACN.

Conclusion: The CACN-L has the potential to fulfill the apparent needs of its users. It allows rapid communication regarding clinical and research issues while linking Canada's geographically separated pediatric neurologists.

Membership of the CACN-L (CACN-L@AC.DAL.CA): K Gordon, moderator (KEGOR@AC.DAL.CA), P Camfield, J Dooley, E Wirrell, Halifax, NS, D Buckley, St John's, NF, D Meek, St John, NB, P Huang, W Logan, R Munn, Toronto, ON, $S$ Levin, London, ON, C Adams, H Darwish, Calgary AB, E Gauk, Edmonton, AB, M Connolley, H Dunn, K Farrell, A Hill, E Roland, Vancouver, BC, J Reggin, A Prasad, A Wilfong, USA.

\section{PAEDIATRIC NEUROSURGERY}

\section{P-047}

Cervical Sensory Evoked Potentials to Tibial Nerve Stimulation - A Retrospective Study of a Paediatric IntraOperative Population

\section{MONCADA, M.J. TAYLOR (Toronto, Ontario)}

Somatosensory evoked potentials (SEPs) elicited by stimulating the tibial nerve (TN) at the ankle have been useful for assessing neural conduction in the cauda equina and posterior spinal columns in clinical testing and during spinal surgical procedures. Popliteal fossa, lumbar spine and cortical responses have been utilized to assess neurological function from lower limb stimulation. TN responses recorded over the cervical spine have not been widely studied as these are difficult to record in most awake subjects. In this study, cervical responses to TN stimulation in anaesthetized patients are retrospectively assessed in a paediatric population. Cervical waveforms from fifty-six neurologically intact patients in three age groups - 9-11 years, 12-14 years and 15-18 years - who received TN SEP monitoring during spinal surgical procedures were measured. The waveform is bifid and the latencies of both negative peaks were measured. The latencies of the first and second peaks respectively for each age group are as follows: $9-11=25.11 \mathrm{~ms}( \pm 3.42), 28.37 \mathrm{~ms}( \pm$ $3.59 ; 12-14=26.02 \mathrm{~ms}( \pm 3.33), 28.68 \mathrm{~ms}( \pm 3.45) ; 15-18=$ $26.67 \mathrm{~ms}( \pm 2.49), 29.48 \mathrm{~ms}( \pm 2.26)$. Contrary to the primary cortical response to TN stimulation, which shows an increase in latency with increases in height in the teenage years, the cervical response presents a relatively stable latency throughout this age period. This cervical response has proven invaluable for intraoperative monitoring of paediatric patients. As children are more susceptible to a loss of the cortical response with anaesthesia, recording cervical responses to $\mathrm{TN}$ stimulation provides the only alternative technique to monitor SEPs in most children during surgery.

\section{P-048}

Relationship Between Fatty Filum, Conus Position and Tethering of the Spinal Cord as Revealed by MR Spinal Cord Motion Analysis

E.T. Kiriakopoulos, A. Mehta, M.L. Wood, C.H. Tator, M.C. FeHlings, D.J. Mikulis (Toronto, Ontario)

Purpose: To assess the importance of a fatty filum and low conus position in predicting spinal cord tethering.

Materials and Methods: Spinal cord motion was measured at the C3 level in 12 patients with fatty fila \pm low conus and in 5 patients without fatty fila and low positioned conus. Conus tip postion below L2 was considered abnormal. The pulse sequence used was a conventional ECG gated 2D phase contrast gradient echo sequence with interleaved flow sensitive and flow compensated components. Following cardiac systole, 40 measurements were obtained at $15 \mathrm{msec}$ intervals. Velocity sensitivity was set to $20 \mathrm{~mm} / \mathrm{sec} \Delta \mathrm{V}$, calculated as the change in velocity from the first caudal peak to the first cranial peak. Any patient with a $\Delta \mathrm{V}$ $<8 \mathrm{~mm} / \mathrm{sec}$ was considered tethered (two SD beyond the mean). Normal $\Delta \mathrm{V}$ is $13.1 \pm 2.6 \mathrm{~mm} / \mathrm{sec}$ (one SD).

Results: In patients with a fatty filum and low conus, spinal cord motion analysis indicated tethering (4/4). Surprisingly, patients with a inferiorly positioned conus and no fatty filum, who by imaging alone would be considered high probability for cord tethering, showed no evidence of tethering (0/5). $25 \%$ of patients with a fatty filum and abnormally positioned conus had tethered cord (2/8). Finally, we have never seen a case of abnormal cord motion in a patient with normally positioned conus and normal filum.

Conclusions: Spinal cord motion studies appear to add discrimination to the anatomical indicators of spinal cord tethering.

\section{P-049}

\section{Intratumoral Bleomycin for Treatment of Cystic} Craniopharyngiomas in Children

\section{P. SteinBor (Vancouver, British Columbia)}

Background: Instillation of bleomycin into craniopharyngioma cysts has been used to thicken the cyst wall prior to attempted surgical resection of the tumor, but has not been recommended as a definitive treatment.

Methods: We report one child with a cystic craniopharyngioma, in whom intratumoral bleomycin resulted in dramatic resolution of the tumor cyst, such that it was elected to observe the child instead of proceeding to surgical resection, and two other children, who are currently being treated similarly. Bleomycin 2mg. was instilled three times per week via a subgaleal reservoir attached to a catheter, whose tip was within the tumor cyst.

Results: The treatment was easy to perform, was well tolerated, and had no side effects. Following treatment with bleomycin in the first patient the tumor cyst gradually decreased until no cyst was evident. The tumor has remained stable after 15 months.

Conclusions: We conclude that intratumoral bleomycin may 
be appropriate as the initial treatment of children with cystic craniopharyngiomas: it may delay or obviate resection of the tumor and the associated complications; and if the tumor does progress, the prior use of bleomycin probably does not increase the risk of tumor resection or make total resection less achievable.

\section{P-050}

\section{Intracranial Ependymomas in Childhood}

\section{W. ZiAI, S.T. Myles, M.G. Hamilton (Calgary, Alberta)}

Background: The best management for children with intracranial ependymomas remains uncertain because outcome is so poor.

Method: A retrospective, population-based cancer registry survey was performed to assess the clinical features and treatment courses of patients, age $\leq 18$ years, with intracranial ependymoma.

Results: There were 18 male and 7 female patients. The mean age at diagnosis was 4.9 years (range 6 weeks to 18 years). The mean duration of symptoms was 1.9 months. The tumour site was infratentorial in 18 patients. Gross total and near total $(>90 \%)$ resection was performed in $41 \%$ of patients. The operative mortality in this series was $8 \%$. Three patients had anaplastic tumours. Sixteen patients had postoperative radiation, 6 chemotherapy. There were 17 deaths, all related to tumour recurrence or progression at the primary site $(1.8 \pm 1.5$ years). Eight patients remain alive (5.9 \pm 5.1 years), of whom 4 have been recurrence free for longer than 5 years. The mean follow-up for all patients was $3.2 \pm 3.8$ years. The overall 5 year survival rate was $20 \%$. Excluding three patients who died perioperatively, the 5 year survival rate among the remaining 22 patients was $36 \%$. Poor prognostic factors include age less than 6 years $(\mathrm{p}=0.011)$, and less than gross total resection at the initial surgery $(\mathrm{p}=0.015)$.

Conclusion: Young age is a very poor prognostic indicator in patients with intracranial ependymoma. Gross total removal of these tumours should be attempted when technically feasible. The failure to improve survival data with current adjuvant therapy protocols indicates a need for more intensive post-operative treatment approaches especially for "poor-risk" patients.

\section{P-051}

Association of Pleomorphic Xanthoastrocytoma with Neuronal Hamartomas and Cortical Dysplasia: Report of Three Cases

\section{N. Duggal, B. Lach, V.F. DaSilva, B.G. Benoit (Ottawa, Ontario)}

Background: Pleomorphic xanthoastrocytoma is a superficially located astrocytic neoplasm characterized by cellular pleomorphism, variable xanthomatous changes and a relatively benign clinical course. The exact histogenesis is still a controversial issue. We present three cases in which PXA was found in association with either a neuronal hamartia or a mature gangliocytic tumor (GT).
Methods: The clinical histories for the three cases were reviewed. Light microscopy, immunohistochemistry and electron microscopy techniques were used to confirm the pathological diagnosis.

Results: In all cases the PXA component was GFAP positive and met the necessary histological criteria for diagnosis. Associated with the PXA, a neuronal hamartia was found in one case while a component of mature GT was present in the two remaining cases. All patients described a long history of seizures predating the surgery.

Conclusions: The longstanding history of seizures and at least one radiologically documented silent lesion several years before surgery suggest that PXA in our patients may have developed in a hamartomatous GT or preexisting cortical dysplasia. Our findings along with other reported cases suggest that PXA may arise from either a subclass of astrocytes histogenetically and topographically associated with neurons or from multipotential precursor cells common to neurons as well as astrocytes. It is not inconceivable that some cases of PXA may represent a ganglioglioma overshadowed by its astrocytic component.

\section{P-052 Withdrawn}

\section{P-053}

Massive Posterior Dural Arteriovenous Malformation Presenting with Cardiac Failure in a Neonate: Successful Treatment with Endovascular Therapy

A. RANGer, S.P. Lownie AND J.M. Rogers (London, Ontario)

Background: Dural arteriovenous malformations (AVMs) involving the posterior dural sinuses in children are extremely rare. Of the six cases diagnosed in the neonatal period, all presented with severe cardiac failure, with a mortality rate of 67 per cent.

Methods: We present the case of a newborn male who developed acute progressive cardiac failure at 10 hours of age, requiring intubation, inotropic support and nitric oxide therapy. Ultrasound and CT scan of the head revealed a huge vascular mass occupying one-half of the anteroposterior diameter of the head. Angiography showed a massive dural AVM supplied by markedly enlarged middle meningeal, occipital and vertebral muscular branches. Percutaneous endovascular embolization of the malformation was performed in two stages, using acrylic glue.

Results: Dramatic reduction of arteriovenous shunt flow was accomplished. By 5 weeks of age, the baby was extubated and transferred out of the ICU. At 2 months, he was discharged from hospital. CT scan at 1 year showed a small residual pouch about $2 \mathrm{~cm}$ in diameter. At 16 months of age, he is developmentally normal.

Conclusion: Minimally invasive treatment of this rare and potentially lethal condition led to a cure of the cardiac failure and allowed for normal neurological development in this infant. 


\section{P-054}

Complications of Growing Skull Fracture of Childhood - A Case Report

F. Maroun, C. Goodyear, J.C. Jacob, S. Bridger and G. MURRAY (St. John's, Newfoundland)

A 4 year old boy with growing skull fracture is presented. The complicated clinical course requiring several surgical procedures is emphasized. Two distinct categories of growing skull fracture in childhood will dictate the type of management:

1) the active form with raised ICP, mass effect on CT, progressive separation of bone edges with a tense bulging between them requiring shunting.

2) the arrested form with normal ICP and a slack sunken gap between the bony edges showing evidence of bone regrowth at fracture site.

Recurrence has been reported with failure to secure tight dural closure or failure to correct the raised ICP.

\section{P-055}

Intervertebral Disc Herniation in the Pediatric Population Analysis of 23 Cases

F. Maroun, J.C. Jacob, L. Michael, G. Murray (St. John's, Newfoundland), L. BADEJo (Kearney, Nebraska)

The authors report on their series of 23 patients who underwent surgery for intervertebral disc herniation. The ages ranged from 9 to 17 years. Many other series include cases up to 20 years inclusive. The majority of cases being in the 18 to 20 year old category. Presentation, investigation, management and outcome are discussed.

\section{P-056}

\section{The State of Neuroendoscopy in Canada}

\section{M.G. Hamilton (Calgary, Alberta)}

Background: Neuroendoscopy is a relatively new subspecialty neurosurgical technique in Canada and treatment indications are now starting to be defined.

Methods: A telephone survey of 15 neurosurgical centres (cities) in 9 provinces was completed to determine if neuroendoscopy was being performed, on what type of patients, and with what types of neuroendoscopes.

Results: Eight centres (5 provinces) have neuroendoscopy programs, although only 4 centres ( 3 provinces) have performed 25 or more procedures (excluding shunt placement). Most of these centres have both rigid and flexible endoscopes. Seven programs have no endoscopy equipment or have not yet performed a procedure. Of the 8 centres with active neuroendoscopy, 6 work with both adults and children (majority of procedures performed in children in all centres), and one each with only adults or children. In 4 of the 6 programs treating both adults and children, pediatric neurosurgeons perform the adult neuroendoscopic procedures.
Conclusion: Neuroendoscopy is currently limited to half of the Canadian provinces, and is in an early state of development in half of the neuroendoscopic programs. It is recommended that a working group be created to help guide the development and growth of neuroendoscopy in Canada. This group should aim to establish minimum neurosurgical training and equipment standards and help define indications for neuroendoscopy on both adults and children.

\section{PERIPHERAL NERVE AND MUSCLE}

\section{P-057}

\section{Motor Neuronopathy With Type 1 Anti-Purkinje Cell Antibodies (PCA-1)}

S. Khwaja, N. Sripathi, B.K. Ahmad (Detroit, Michigan), V.A. LENNON (Rochester, Minnesota)

Background: PCA is serological marker of ovarian or breast cancer who present characteristically with subacute cerebellar degeneration; $<2 \%$ of ovarian cancer patients have low titre PCA-1 antibodies without neurological signs. A minority of PCA-1 positive patients with gynecologic cancer present with peripheral neuropathy without cerebellar signs (Neurology 4 (Suppl. 4): A320, April 1995). Motor neuronopathy has not previously been reported with PCA-1 seropositivity and ovarian cancer.

Methods: Case report of a unique presentation in a 67 year old woman with ovarian carcinoma and type- 1 anti-Purkinje cell antibodies.

Results: The patient presented with progressive weakness and atrophy of bilateral upper extremities with brisk biceps reflexes and no sensory deficit or signs of cerebellar or craniobulbar muscle involvement. Lower extremities were involved minimally. Stage IIIC ovarian adenocarcinoma was diagnosed. EMG revealed motor neuronopathy with relative sparing of sensory potentials, and severe involvement of upper extremities. Serum was positive for PCA-1 antibodies at 1:61,440 dilution. The patient's IgG bound to a dominant neuronal antigen, $\sim 58$ $\mathrm{kDa}$, as did IgG in sera of PCA-1 positive control patients who had paraneoplastic cerebellar degeneration.

Conclusion: This patient's presentation with paraneoplastic motor neuronopathy, a rare clinical entity, extends the neurologic spectrum associated with ovarian carcinoma and PCA-1 autoantibodies.

\section{P-058}

Is the Ulnar Nerve Compressed at Guyon's Canal in Patients with Carpal Tunnel Syndrome?

\section{J. De Léan, H. David (Québec City, Québec)}

Background: Patients with carpal tunnel symptoms may have paresthesia in the little finger. The underlying mechanism has yet to be established. Compression at Guyon's canal is often presumed and may even lead to surgical release. 
Methods: Group I was for asymptomatic hands (72 right and 70 left), group II for symptomatic hands with clinical and definite electrophysiological evidence of carpal tunnel syndrome (CTS) without symptoms in the little finger (136 right and 92 left), and group III for hands with CTS having little finger paresthesia (110 right and 95 left). Patients with associated disorders were excluded. Median distal motor latency, ulnar distal motor latency (UDML), transcarpal median sensory conduction, ulnar distal sensory conduction velocity (UDSCV), ulnar sensory nerve action potential amplitude (USNAPA) and duration (USNAPD) were measured.

Results: Ulnar nerve conduction abnormalities were found only in seven hands (group III) due to subclinical polyneuropathy (three hands), ulnar nerve entrapment at the elbow (three hands) and thoracic outlet syndrome (one hand). When these abnormal values were excluded, one-way analysis of variance failed to reveal a statistically significant difference $(p>0.05)$ among the three groups, in both right and left UDMLs, UDSCVs, USNAPAs (adjusted for the effect of age) and USNAPDs.

Conclusions: Our study of ulnar nerve conduction studies at the wrist revealed only some abnormality related either to polyneuropathy or more proximal entrapment. These findings do not suggest concomittant ulnar nerve entrapment at Guyon's canal in patients with CTS whether or not they have paresthesia in the little finger.

\section{P-059}

\section{Dose-Related Alcoholic Polyneuropathy}

M.L. D' Amour, S. Brissette, J. Lavole, R.F. Butterworth (Montreal, Quebec)

Background: Alcoholic polyneuropathy is well described in severe alcoholic patients. However, studies have not looked at moderate drinkers. We wanted to know if moderate drinkers had also signs of polyneuropathy, since this may have some significance with regard to physiopathology. Also, what is considered to be a more benign habit, may in fact not be so.

Methods: We defined moderate consumption as an alcohol intake of 2-6 drinks/day (60-90 gms alcohol/day) and severe consumption as more than 6 drinks/day ( $>100 \mathrm{gms}$ alcohol/day) over a period of several years. At the present time, we have been able to evaluate 27 control, 23 moderate and 28 severe subjects with no other causes of polyneuropathy. They were in the same age group. Each had a clinical, electrophysiological and biochemical assessment, including thiamine status. Electrophysiologically they had 7 nerve conduction studies and needle EMG if appropriate. Thiamine status was expressed as the effect of thiamine pyrophosphate (TPP) on their transcetolase level, a more sensitive index of thiamine effect deficiency.

Results: Nerve conduction velocities in $\mathrm{m} / \mathrm{sec}$ in control, moderate and severe subjects respectively were as follows : Sensory: Median 62, 55, 54 Ulnar 60, 58, 52 - Sural 51, 49, 47; Motor: Median 59, 56, 54 - Ulnar 60, 60, 55 - Peroneal 48, 48, 46 Posterior tibial 46, 46, 46. Control subjects had a normal TPP effect $<15 \% ; 2 / 23$ of the moderate and 10/28 of the severe sub- jects had values $>15 \%$. Moderate subjects had a normal clinical examination, although a few had minimal signs of polyneuropathy.

Conclusions: It was interesting to note that sensory nerves and motor median nerve conduction velocities deteriorate progressively in the moderate and severe group, and seemed in proportion to the quantity of alcohol ingested. Thiamine deficiency increased between the moderate and severe group. Could there be a correlation between nerve conduction slowing and thiamine deficiency? Further analysis will have to be done. This may have pathophysiological and functional significance.

\section{P-060}

\section{Nerve Biopsy Findings in Chronic Inflammatory Demyelinating Polyneuropathy}

\section{A.F. HAHN, H. KIRK, R. JeUn (London, Ontario)}

Background: Nerve biopsies were studied in 26 patients with a clinical diagnosis of CIDP of less than 2 years duration.

Methods: The pathology was assessed in epon $1 \mu \mathrm{m}$ sections (26) and by teased fibre analysis (26). Endoneurial lymphocytes, macrophages and class Il antigen expression, deposition of IgG, $\operatorname{IgM}$ and $\mathrm{C}_{3}$ were studied from immuno-stained serial cyrostat sections (21).

Results: Variable degrees of chronic and ongoing macrophage-mediated de/remyelination and secondary axonal degeneration were observed. Teased fibre studies were important for an accurate assessment. In some cases the changes in distal sensory nerves did not correlate well with the severity of clinical symptoms, suggesting predominant proximal disease. B cell infiltrates were rarely seen, yet $T$ lymphocytes $\left(C_{4}>C D_{8}\right)$ were readily identified and correlated well with the degree of demyelination, macrophage invasion and increased expression of class II antigen. Endoneurial deposition of IgM/IgG and the complement component $\mathrm{C}_{3}$ were shown on individual myelin sheaths in some cases.

Conclusion: The findings are consistent with the concept of an immune-pathology mediated by cellular and humoral factors.

\section{P-061}

\section{Fibrinoid Necrosis in Vasculitic Neuropathy}

\section{WhITE (Kingston, Ontario), J. MAGUIRE (Hamilton, Ontario)}

Background: Vasculitis was previously defined pathologically by full thickness inflammation of blood vessel walls with fibrinoid necrosis. This retrospective study assessed the importance of fibrinoid necrosis in vasculitic neuropathy.

Methods: Cases of vasculitic neuropathy were retrieved from 302 nerve biopsies examined at the Hamilton General Hospital from 1990 to August 1995. Nerve biopsies were studied for: full thickness inflammation of vessel walls, fibrinnoid necrosis, location of affected vessel (epineurium, perineurium, and endoneurium) and vessel calibre. When available, concomitant muscle biopsies were studied.

Results: After careful review, twenty-five nerve biopsies 
were found to display full thickness inflammation of blood vessel walls. Small epineural vessels were most affected (24 out of 35). Fibrinoid necrosis was identified in six, affecting vessels greater than 100 microns in calibre. Muscle biopsies were postive for vasculitis in four out of eleven cases.

Conclusions: Of the twenty-five nerve biopsies showing full thickness inflammation of vessel walls, fibrinoid necrosis was variable finding affecting only vessels greater than 100 microns in calibre. The presence of fibrinoid necrosis in only large vessels may indicate different pathogenetic mechanisms, depending on vessel calibre.

\section{P-062}

\section{Early Onset Axonal Neuropathy in a Cree Kindred}

N.J. Lowry, D.H. George, R.J.B. MaCaulay, P.S.W. Davidson (Saskatoon, Saskatchewan)

Background: Hereditary Motor Sensory Neuropathy (H.M.S.N.) types I and II are now well characterized. In 1991 a more severe childhood axonal neuropathy was distinguished from H.M.S.N. II.

Methods: We have followed and studied a large pedigree of Cree children who have inherited this condition in a dominant fashion with variable penetrance.

Results: All children had severe atrophy of all muscles below the knee by 5 years of age; by 10 years atrophy of the small muscles of the hand was apparent. There was loss of tendon reflexes and progressive loss of vibration and position sense in the lower limbs. Nerve conductions showed low or absent sensory conductions with normal motor velocities; in severely affected lower limbs all conductions were absent. Sural nerve biopsies of three individuals showed similar features: severe loss of myelinated axons, preferentially affecting large diameter fibres; absence of active axonal degeneration; paucity of regenerative axonal sprouts; and absence of features of segmental demyelination and onion-bulbs formation.

Conclusion: This is a rare axonal neuropathy which is distinct from H.M.S.N. type II and more severe in degree.

\section{P-063}

Amplitude of Sural and Radial Sensory Nerve Action Potential in Orthodromic and Antidromic Studies in Children

\section{J. Melandrez, L.J. MacMillan, J. VaJsar (Toronto, Ontario)}

Background: Several previous studies have reported that the amplitudes of the sural and superficial radial nerve action potentials (SN, SRN, SNAP) are larger with antidromic than with orthodromic recordings. However, this difference has not been documented in children. This study evaluates the amplitudes of SN and SRN SNAPs obtained with antidromic and orthodromic recordings in children and compares the data with findings in adults.

Methods: SN and SRN of 10 neurologically normal children (age 5 to 17) and 7 adults were studied with surface stimulation and recording. Orthodromic and antidromic recordings were performed by reversing the position of the stimulating and recording electrodes. Peak to peak SNAP amplitudes were measured and analyzed.

Results: The amplitudes of SRN SNAP obtained with antidromic techniques were significantly larger in the children than in the adults. The mean values were 48.0 and $57.8 \mu \mathrm{V}$ in antidromic and 29.5 and $44.1 \mu \mathrm{V}$ in orthodromic recordings $(\mathrm{p}<$ .05). Similarly, the SN SNAPs were larger in antidromic than orthodromic recordings: 25.4 and $19.5 \mu \mathrm{V}$ in children and 17.3 and $13.6 \mu \mathrm{V}$ in adults.

Conclusions: Consistent responses were obtained with both techniques. However, the antidromic technique was superior to the orthodromic technique because it was more comfortable and the amplitude of response was greater.

\section{P-064}

Skeletal Muscle Fibre Type Transformation Following Spinal Cord Injury

R. Burnham, T. Martin, G. Bell, I. Maclean (Edmonton, Alberta)

Background: Based on qualitative ATPase staining characteristics, previous reports have described the upper motor neuron paralysed muscles of individuals with traumatic spinal cord injury of several years duration to be predominantly fast fatiguable type 2 . This fibre type transformation partially explains the the common problem of early fatiguability of muscle when rehabilitation efforts using functional electrical stimulation (FES) are attempted. Muscle fibre typing based on antibodies to specific myosin heavy chain (MHC) isoforms provides identification of type 1 , type 2 , and fibres co-expressing both myosin isoforms. The purpose of this study was to describe MHC expression in spinal cord injury paralysed muscle over a wide range of times post-injury.

Methods: MHC expression was analysed on 17 vastus lateralis biopsies of 12 individuals with SCI of a duration ranging from 2 weeks to 18 years.

Results: Within 6 weeks post-injury the percentage of type 1 fibres started declining and the percentage of co-expressing fibres increased. This trend peaked at approximately 1 year post-injury after which the percentage of co-expressing fibres dropped and the type 2 fibres prevalence increased.

Conclusions: Type 1 fibre disappearance from $\mathrm{SCl}$ paralysed muscle starts within weeks post-SCI and goes through an intermediate stage of co-expressing slow and fast myosin before becoming predominatly fast myosin muscle. Intervention to minimize the effects of this transformation, like FES, may best be applied early post-injury. 


\section{P-065}

Myopathic Motor Unit Action Potentials (MUAP's) In Inflammatory Myopathy

\section{R.Z. Kern, A. Alex (Mississauga, Ontario)}

Purpose: We attempted to identify myopathic MUAPs in seven patients with biopsy-proven inflammatory myopathy by measuring MUAP duration and area-amplitude ratio (thickness).

Methods: MUAPs were collected from the vastus lateralis and triceps muscles using a semi-automated computer-assisted method (Advantage, Clark-Davis). The technique utilizes template-matching of low threshold MUAPs selected by amplitude. Age-matched control MUAPs were collected and reference values established using mean $+/-2$ s.d.

Results: Thirty-four percent of triceps and eleven percent of vastus lateralis MUAP $\mathrm{s}$ were identifed as myopathic by MUAP duration or thickness. The triceps and vastus lateralis mean MUAP duration and thickness were significantly reduced compared to control MUAPs $(p<0.001)$, however the vastus lateralis mean MUAP values were not significantly lower than control MUAPs ( $p>0.1$ ). Most myopathic MUAPs were identified by reduced MUAP duration and very few thin MUAPs had normal durations.

Conclusions: Only a small number of MUAPs could be classified as myopathic. These were seen more frequently in the triceps than the vastus lateralis. Myopathic MUAPs were identified primarily by duration criteria since very few thin MUAPs had normal duration. These observations may be disease-specific. More likely they reflect the considerable overlap of MUAP parameters in patients and controls.

\section{P-066}

\section{A Comparison of Open Needle Muscle Biopsies: Review of} 250 Cases

L. Stevens, M. Tarnopolsky, S. Fawcett, J. Maguire (Hamilton, Ontario)

Background: Over 5000 needle muscle biopsies (NMB) have been performed for neuromuscular diagnosis and research in Hamilton over the past 20 years. This retrospective review compared the diagnostic yield, complication rates and costs of NMB to open muscle biopsies (OMB).

Method: All 250 muscle biopsies performed in the previous two years were reviewed and assessed for their diagnostic yield. We evaluated complications in $>50 \%$ of these clinical biopsies and all of the 3586 research biopsies. Costs were calculated using the 1992 OHIP schedule of benefits. Results were analyzed using Chi square analysis.

Results: A specific neuromuscular diagnosis could be established on the basis of muscle biopsy in $74 \%$ of NMB and $73 \%$ of OMB (ns). Nonspecific pathologic features were seen in $20 \%$ of NMB and $6 \%$ of $\mathrm{OMB}(\mathrm{p}<0.05) .5 \%$ of $\mathrm{NMB}$ and $11 \%$ of OMB were normal (ns). $5 \%$ of NMB and $9 \%$ of OMB yielded unsatisfactory samples (ns). Few complications were reported in either group. 12 minor complications seen in the 3586 research biopsies resolved within four months. Costs were much greater for OMB than for NMB.
Conclusions: NMB and OMB are equivalent in diagnostic yield with low complication rates. The decreased cost and increased convenience to the patient would favour NMB as the procedure of choice.

\section{P-067}

\section{Heart Involvement in Myotonic Dystrophy. A Clinical Follow-up of 71 Patients}

A. Mammarella, P.V. Fragola, V. Paoletti, G. Antonini, M. Borzi, S. Morino, F. Gragnani, A. Musca (Rome, Italy)

Background: Impairment of conduction system is the hallmark of heart involvement in myotonic dystrophy and may influence the prognosis remarkably. Serious arrhythmias may also occur. Overt myocardial impairment is rare. This study represents an extension of our previous reports on the field.

Methods: Seventy one consecutive patients (47 men and 24 women, mean age $37 \pm 14$ years) were enrolled in a follow-up study including serial electrocardiographic, Holter monitoring, and echocardiographic examinations. Of the 71 patients, 30 had mild disease, 23 moderate disease, and 18 severe disease. Mean follow-up was $56 \pm 38$ months (2 to 192).

Results: At entry, 22 patients $(31 \%$; 4 with mild, 8 with moderate, and 10 with severe disease had $\geq 1$ of the following conduction defect: first-degree atrioventricular block (17 cases), nonspecific intraventricular conduction delay (2 cases), bundle branch block ( 7 cases), and hemiblocks ( 3 cases). A variety of atrial and/or complex ventricular arrhythmias occurred in 7 patients. No patient had echocardiographic signs of ventricular dysfunction and none complained of cardiac symptoms. During follow-up 15 of the 22 patients with conduction defects at baseline had a spectrum of additional disturbances suggesting deterioration of conduction system disease. Serious defect appeared de novo in 6 patients with prior normal study. Of the 28 patients with conduction defects, 9 (4 with moderate and 5 with severe disease) required pacemaker insertion because of the occurrence of syncope and prolonged ( $>55 \mathrm{~ms}$ ) His-to-ventricle interval at electrophysiologic study. A 17-year-old boy with mild disease and with chronic atrial fibrillation and 1 episode of nonsustained ventricular tachycardia on Holter died suddenly while awaiting electrophysiologic study. Three patients died of noncardiac cause.

Conclusions: There is no certain way to predict the course of conduction system disease in myotonic dystrophy. Therefore, all patients should have close cardiologic observation, regardless of the severity of the neuromuscular disease, electrocardiographic findings, and symptomatology.

\section{P-068}

\section{Tarsal Tunnel Syndrome - Case Report}

\section{R.J. Broome, J.D. Brown, B.V. Watson (London, Ontario)}

Background: A 37 year old man had a 2 month history of progressive numbness in all digits and the entire sole of his left 
foot which began while inflating his left skate boot with two pumps located below the medial and lateral malleolli.

Methods: Standard surface orthodromic motor and sensory nerve conduction studies and needle electromyography were performed 2 and 3.5 months following symptom onset.

Results: Sensory conduction across the tarsal tunnel in the left medial and lateral plantar nerves was reduced by $28 \%(36.6 \mathrm{~m} / \mathrm{s})$ and $33 \%(36.2 \mathrm{~m} / \mathrm{s})$, respectively, when compared to the right side; sensory amplitudes were reduced by $87 \%$ ( 1.7 microvolts) and $72 \%$ ( 2.3 microvolts), respectively. Needle studies demonstrated fibrillation potentials and positive sharp waves in the left plantar foot muscles. Repeat studies 6 weeks later revealed no evidence of active denervation and significant improvement in the left medial plantar sensory response (1.7 to 7.6 microvolts).

Conclusions: We describe an unusual presentation of tarsal tunnel syndrome due to compression by increased boot pressure. Substantial improvement in symptoms and neurophysiological findings were observed after the patient stopped using this inflatable skate boot.

\section{P-069}

\section{Atypical Symptoms in Patients With Carpal Tunnel Syndrome}

\section{S. GuPTA, T.J. Benstead (Halifax, Nova Scotia)}

Background: Patients with clinical suspicion of carpal tunnel syndrome (CTS) sometimes report symptoms of pain, numbness, and tingling outside the median nerve distribution. This questionnaire-based study was designed to improve understanding of such atypical symptoms reported by CTS patients.

Methods: Patients with clinical suspicion of upper limb neuromuscular lesions were divided into those with EMG evidence of CTS, and those without. CTS patients with superposed nerve abnormalities were excluded. Motor and sensory symptoms were assessed in this homogeneous group of CTS patients.

Results: Over 50\% of patients with exclusive EMG criteria for CTS reported parasthesias or numbness over the whole hand or ulnar or radial nerve distributions. Moreover, symptoms were not restricted to the hand alone, but were found proximal to the site of the putative lesion, in the whole arm. Nocturnal pain and numbness were predictive of positive EMG evidence of CTS.

Conclusions: Atypical sensory symptoms are common in CTS. For accurate diagnosis it is necessary to become aware of the atypical/non-classical symptoms. Simple and sensitive diagnostic tools such as reports of nocturnal pain and numbness should be important adjuncts for the clinical assessment of this common disorder.

\section{P-070}

\section{Progressive Polyradiculopathy in AIDS: Electro- physiological and MRI Studies}

\section{H. Al-Rahi, M. VeilleuX (Montreal, Quebec)}

Background: Progressive polyradiculopathy (PP) is a rare neuromuscular complication of HIV infection usually heralded by low CD4 counts and coexisting opportunistic infections. Prompt diagnosis and treatment may have a favorable impact on quality of life in these patients. Clinical course is usually uniform and CSF findings distinctive. If the clinical course or CSF findings are atypical, MRI of the spinal cord may provide evidence suggestive of PP.

Methods: All AIDS patients admitted to the Montreal General Hospital with diagnosis of peripheral neuropathy were retrospectively identified and their charts reviewed for clinical presentation, laboratory, electrodiagnostic and imaging studies.

Results: We found only 2 AIDS patients with a diagnosis of PP. Both had symmetrical involvement of the legs, AIDS for less than 3 years and associated opportunistic infections. CD4 counts were 50 and $184 / \mathrm{cc}^{3}$. CSF studies revealed polymorphonuclear pleocytosis, high protein and low glucose. On electrodiagnostic studies, there was extensive denervation in leg muscles. MRI of the thoracic and lumbar spine showed thickening and enhancement of the cauda equina and conus medullaris.

Conclusion: MRI of the spinal cord may provide evidence to support a diagnosis of PP in AIDS patients with atypical clinical presentation or CSF studies.

\section{P-071}

\section{A Randomized Trial of Bemethyl in Progressive Muscular Dystrophies}

\section{Pustozerov, L.A. Saikova (St. Petersburg, Russia)}

Background: Bemethyl (benzoxazepin-methyl-benzomedazole, CGS 9343B, Kiev, Ukraine) was synthesized in the 70-s as an acktoprotective agent ("aktus" - movement). The agent stimulates gluconeogenesis and thus improves the performance in physical activity in animal models and pilot studies on human volunteers.

Methods and Results: A prospective randomized trial of bemethyl was performed on patients with muscular dystrophy who had not had systematic treatment previously. We present the data on the following groups: Duchenne, Erb, Landousy, scapuloperoneal and other types of progressive musclar dystrophies. A total of 443 patients were enrolled. There was no placebo group and patients who received bemethyl were compared to those who received standard treatment for muscluar dystrophy employed in this country. This includes vitamins, ATP, vasoactive agents, anabolic hormones, and massage for 30 days. The patients were randomly assigned to bemethyl $(n=268)$ and standard treatment $(n=175)$ groups. The patients were divided into the mild, moderate, and severe groups based on the Movement Capacity Index (MCI) scores similar to $\mathrm{ADL}$ measurements (normal $\mathrm{MCI} .=100$ points). Improvement was noted in all treatment groups and disease categories for mild and moderate involvement. In the severe group bemethyl produced significantly more improvement when baseline $\mathrm{MCI}$ scores were compared to that at discharge: $22.3 \pm 2.0$ vs $28.8 \pm 1.9(\mathrm{n}=51, \mathrm{p}=0.0001)$, and control $21.3 \pm 2.3$ vs $21.5 \pm 2.8(\mathrm{n}=35, \mathrm{p}=0.74, \mathrm{~ns})$.

Conclusions: We conclude that bemethyl is as effective as other traditionally used treatment strategies in patients with mild and moderate muscular involvement. In severely disabled patients with muscular dystrophy bemethyl improves movement capacity better than the other treatments. 
Acknowledgement: This poster is presented by the authors via conference call moderated by Drs Marek Gawel and Andrei Alexandrov. The presentation is jointly sponsored by STEMC, a technology transfer project of Bimman Inc., and by Caritan International Assistance Charity, a registered Canadian charity helping people in the Newly Independent States to help themselves to become economically independent.

\section{P-072}

Orbital Myositis Without Local Evidence of Inflammation: a Case Report

\section{S. Meckling H. Z. Darwish (Calgary, Alberta)}

Background: Orbital myositis(OM) is an idiopathic inflammatory disorder of the extra-ocular muscles. It is almost invariably associated with some combination of ocular chemosis, proptosis, ocular pain, and periorbital or lid edema, along with ophthalmoplegia. We report a case without these typical features.

Methods: Case report.

Results: A 12-year-old boy presented for evaluation of persistent frontal headaches after minor head trauma without LOC. Examination demonstrated painful ophthalmoplegia with limitation of adduction of the right eye as well as limited upgaze. There was no evidence of proptosis, chemosis, or periorbital edema. A Tensilon test was negative. Laboratory investigations including $\mathrm{CBC}, \mathrm{ESR}, \mathrm{CK}, \mathrm{TSH}$, and antithyroid antibodies were negative. A CT of the head showed an enlarged right medial rectus muscle. This was confirmed on MRI which demonstrated other muscle involvement. No specific therapy was given and spontaneous resolution of symptoms, signs, and MRI findings occured. He continues well at one year.

Conclusions: OM should be considered in all cases of painful ophthalmoplegia, even if local evidence of inflammation is lacking. MRI is useful in the diagnosis and follow-up of this condition. Spontaneous resolution can occur.

\section{P-073}

Colchicine Neuromyopathy with Early Defect of Neuromuscular Transmission

A. Boyle, M.Yeung, P. Siemens, A. Shuaib (Saakatoon, Saskatechwan)

Background: Colchicine causes multisystemic toxicity. Neurologic involvement includes neuromyopathy and encephalopathy.

Method: We report the case of a woman with mild chronic renal failure, recently commenced on colchicine, who developed progressive weakness, peripheral sensory disturbance and encephalopathy.

Results: Initial electrophysiological testing revealed a decremental response at slow and fast rates of repetitive stimulation in several motor nerves, suggesting a defect of neuromuscular transmission. Serial nerve conduction testing and electromyography demonstrated the evolution of neuropathy and myopathy, but resolution of the apparent defect of neuromuscular transmis- sion. She was unresponsive to edrophonium chloride (Tensilon ${ }^{\circledR}$ ) or pyridostigmine bromide (Mestinon $\left.{ }^{(}\right)$. Anticholinesterase antibodies were negative.

Conclusion: Neuromuscular transmission defects have not been previously reported in colchicine neuromyopathy.

\section{P-074}

\section{Cyclosporin A Neurotoxicity in a 55-Year old Liver} Transplant Patient

F. Souchon, S. Ledoux, D. Marleau, S. Fontaine, L.H. LEBRUN (Montréal, Québec)

Case history: We present a 55 year old alcoholic cirrhotic patient who received a liver transplant. On post-op day 18, he developed headaches and tremor, followed over a few hours by a severe pseudobulbar palsy with anarthria, aphagia, quadriparesis and corticospinal signs. A complete investigation excluded central pontine myelinolysis, and any infectious or vascular insult. After Cyclosporin A (CSA) doses were reduced, the patient showed substantial recovery and 6 months later he only has a slight ataxia and dysarthria. We believe this is a case of CSA neurotoxicity since we excluded other conditions and patient showed recovery with CSA dose reduction.

Background: CSA, which is used as immunosuppressant in most organ transplants, is neurotoxic. The diagnosis of neurotoxicity isn't easy to make, because it can cause very different manifestations in patients who may already have many different neurologic complications. Moreover, there is no correlation between CSA blood levels and neurotoxicity. It is very important to make the diagnosis, since even when very severe, this condition is almost completely reversible when CSA is reduced or ceased.

Discussion: The higher prevalence of neurotoxicity in liver transplant patients may be in relation with a higher brain susceptibility after numerous episodes of hepatic encephalopathy. Since the risk for neurotoxicity cannot be predicted by CSA levels measurements, toxic metabolites of CSA may be implicated in its pathogenesis. Furthermore, evidence suggests that lower p450 cytochrome levels might explain individual susceptibility.

Conclusion: Further prospective studies of CSA neurotoxicity in transplant patients are clearly warranted.

\section{P-075}

Amyotrophic Lateral Sclerosis Symptom Onset Concurrent with the Use of Cholesterol Lowering Agent Therapy

\section{M.J. Strong, A.F. HahN (London, Ontario)}

Background: Risk factors for the development of amyotrophic lateral sclerosis (ALS) include mutations in the SOD I gene, electrical injury, trauma, and employment within the plastics industry. Although the role of exogenous factors in the etiology of ALS remains controversial, recognition of additional precipitants may provide insight into the pathogenesis of ALS.

Case Reports: We have observed 7 cases of clinically-definite ALS amongst a population of 389 patients in whom 
symptoms onset within 8 to 12 months of the initiation of cholesterol-lowering therapy by pharmacological means. The mean age of symptom onset was $66.2 \pm 6.3$ years. Disease onset consisted of prominent cramping, asymmetric weakness and wasting. Progression ensued in spite of discontinuation of therapy. Five of the 7 patients also had dietary-controlled diabetes mellitus and 3 had hypertension requiring pharmaco-therapy. In all but one, additional medications were utilized, although no other family of medications was common to all patients.

Conclusions: Our observation of $1.8 \%$ of ALS patients utilizing cholesterol lowering agents prior to symptom onset would be within the predicted range for the utilization of these agents within the adult Canadian population (approximately 3.8\%; Merck Sharpe database, 1995). It is most likely, therefore, that the use of these agents has unmasked the symptoms of ALS at an earlier time interval than might have occurred otherwise. Based on these observations, it is recommended that patients developing focal motor deficits, cramps or fasciculations while ingesting cholesterol-lowering agents, who fail to improve following withdrawal of the agent, be fully investigated for a motor neuron disorder. MJS is supported by a MRC Scholarship and by the ALS Society of Canada.

\section{SURGICAL ONCOLOGY}

\section{P-076}

\section{The Role of Platelet Derived Growth Factor in Reactive Astrogliosis}

\section{S. Mukherjee, N. Lau, J. Provias, A. Guha (Toronto, Ontario)}

Injury to the CNS from a variety of insults leads to reactive astrogliosis, involving proliferation and hypertrophy of astrocytes, with increased GFAP expression. Although a normal reparative process, exuberant reactive astrogliosis may have significant clinical sequelae, such as impeding neuronal regeneration, spreading of a seizure focus, and being difficult to differentiate from recurrence of malignant astrocytomas. Platelet Derived Growth Factor (PDGF) is a strong mitogen for astrocytes (which express its cognate receptors) in-vitro, and is expressed in large amounts by neurons. It is hypothesized that injury to neurons would lead to increased mitogenic stimulation of astrocytes by PDGF in a paracrine manner. In this study an animal model of reactive astrogliosis was used to test this hypothesis.

3T3 cells were transfected to over-express PDGF-A or PDGF-B and treated with mitomycin-D, so they could not proliferate but remained metabolically active producing the transfected growth factor. The transfected cells and non-transfected $3 \mathrm{~T} 3$ cells (contralateral frontal lobe) were stereotactically injected into the frontal lobes of syngeneic mice under reproducible conditions. The difference in the number of reactive astrocytes (as determined by counting GFAP expressing astrocytes through the injection site) between the transfected vs. non-transfected frontal lobes were determined at 1 and 2 weeks. At 1 week, PDGF-A stimulation led to significantly larger number of reactive astrocytes in $4 / 5$ mice, compared to only $2 / 7$ mice injected with PDGF-B transfected 3T3 cells. However, at 2 weeks PDGF-B stimulation had caused a more exuberant reactive astrocytosis in 5/7 mice compared to only 1/7 PDGF-A injected mice. These results support the hypothesis that PDGF is an important mitogen for astrocytes in-vivo. The amount and temporal profile of PDGF mediated reactive astrogliosis differs between the PDGF subunits, probably due to differences in their biochemical profiles and their affinity towards the PDGF receptor subunits expressed by the astrocytes.

\section{P-077}

\section{Ras Mediated Proliferation of Human Astrocytoma Cells}

\section{A. Guha, N. Lau, A. Pawson (Toronto, Ontario)}

Malignant astrocytomas are the most common adult human primary brain tumor. They over express PDGF and EGF receptors, activation of which contribute to tumor growth. Oncogenic Ras mutations although present in a large number of human cancers are not prevalent in astrocytomas. Our current work demonstrates that PDGF and EGF receptors expressed by human malignant astrocytoma cell lines are capable of activating Ras. This leads to elevated levels of activated Ras-GTP, contributing to their proliferation.

EGF or PDGF stimulation of four human malignant astrocytoma cell lines, resulted in their cognate receptors to complex with Shc, GRB2 and syp, all of which are involved in activation of Ras. Levels of Ras-GTP were increased in the astrocytoma cell lines and similar to oncogenic Ras transformed control fibroblasts by ${ }^{32} \mathrm{P}$ Ras loading experiments. The Asn17 Ras dominant negative mutant was transfected into the astrocytoma cells to decrease Ras-GTP levels, hence determining if this signal transduction pathway contributed to their proliferation. Pooled growth and colony formation assay showed decreased proliferation in 3/4 astrocytoma cell lines. Stable Asn-17 transfected astrocytoma clones also had decreased growth. Phosphorylation of MAP Kinase, a downstream target of activated Ras, was reduced in the Asn-17 transfected astrocytoma cells, correlating with their decreased proliferation. These results support the hypothesis that the proliferative signal of human malignant astrocytoma cells, which are known to overexpress receptor tyrosine kinases such as the PDGF or EGF receptor, utilizes Ras mediated signal transduction. Pharmacological (i.e., farnesyl inhibitors) and genetic inhibitors of the Ras pathway may therefore be of therapeutic value in these tumors.

\section{P-078}

Concordance Between ERCC-2 Gene Expression as Determined by RT-PCR and BCNU Resistance in Human Glioma Cell Lines

Z.P. Chen, A. Malapetsa, A. McQuillan, D. Marcantonio, S. BRIEN, G. Mohr, L.C. PANASCI (Montreal, Quebec)

Drug resistance to chemotherapy occurs in about $60 \%$ of human brain tumors. Nucleotide excision repair (NER) is 
responsible for DNA repair of DNA lesions produced by some chemotherapeutic agents. One protein of the NER system, ERCC-2 functions as a DNA helicase and may play an important role in drug resistance. In this study, we quantitated ERCC2 gene expression by reverse transcript-polymerase chain reaction (RT-PCR) in five glioma cell lines with variable sensitivities to 1, 3-bis-(2-chloroethyl)-1-nitrosourea (BCNU). ERCC-2 expression obtained by RT-PCR correlated with that obtained using Northern blot analysis $(r=0.886, p=0.046)$. We found significant correlations between BCNU cytotoxicity and ERCC-2 expression as determined by both RT-PCR ( $r=0.962, p=0.009)$ or Northern blot analysis $(r=0.945, p=0.015)$. We have shown that quantitation of ERCC-2 expression with RT-PCR is reliable and implicates ERCC-2 in BCNU resistance in human glioma cell lines. ERCC-2 is a human homologue of Rad-3 which may be involved in recongnition of lesions in DNA. (supported by NINDS NS2230)

\section{P.079}

Neurofibromatosis-2 (NF-2) vs. Sporadic Vestibular Schwannoma: A Clinical, Radiological and HistoPathological Study

\section{A. Alkhani, I. Mckenzie, J. Provias, C. Tator, A. Guha} (Toronto, Ontario)

Bilateral vestibular schwannomas are pathognomic of NF-2, which is an inherited autosomal dominant disorder due to loss of the recently cloned NF2 gene on chromosome \#22, whose gene product is known as schwannomin or merlin. In this study, we examined 8 vestibular schwannomas from 6 NF-2 patients and compared them to 12 unilateral sporadic vestibular schwannomas, matched for age, sex and size of the tumor. There were 4 males and 2 females with NF-2 (16-41 yrs), with an average tumor diameter of $3 \mathrm{c} . \mathrm{m}$. The translabyrinthian approach was used in 3, with the remaining $5 \mathrm{NF}-2$ schwannomas resected via a suboccipital approach. None of the NF-2 patients had preoperative $\mathrm{Cr}-\mathrm{V} 11$ palsy, however, ipsilateral cerebellar signs were present in 5/8 tumors. Facial numbness and I.C.P. related headaches were present in 1 patient each. Post-operatively there was 1 partial and 3 complete facial nerve palsies.

Paraffin sections from the NF-2 and sporadic schwannomas were stained with a panel of antibodies (Ab), to determine if any biological differences were present. Loss of NF-2 gene product was evaluated by staining with Ab-schwannomin. MIB-1 staining was undertaken to evaluate the proliferative index of the tumor cells in the two groups. Expression of Glial Growth Factor and Her-2, both of which have been implicated in growth of schwannomas, was also evaluated. Differences in tumor vascularity between NF -2 and sporadic vestibular schwannomas have been suggested. We examined this issue in our two groups by correlating radiological, intraoperative observations and by staining the tumor sections with Ab-FactorV111 and one of the most potent angiogenic stimulant, VEGF (Vascular Endothelial Growth Factor). Expression of hormonal receptors is another area of controversey, that was evaluated by staining for estrogen and progestrone receptors. The similarities and differences in these biological markers between NF-2 and sporadic vestibular schwannomas will be discussed.

\section{P-080}

Increased Neurofibromatosis 1 Gene Expression In Astrocytic Tumors: Positive Regulation By p21-Ras

D.H. Gutmann, D.K. Mahadeo, M.J. Giordano, D. Silbergeld (St. Louis, Missouri), A. GUHA (Toronto, Ontario)

Fifteen to fifty percent of individuals affected with neurofibromatosis 1 (NF1) develop astrocytic tumors. To determine whether loss of NF1 expression is associated with progression towards malignancy in astrocytomas, twenty eight fresh astrocytoma operative specimens (low and high grade tumors) and ten primary human astrocytoma cell lines were examined for NFI mRNA and protein expression. In all astrocytomas examined, high levels of $\mathrm{NF} 1$ expression were observed relative to normal resting astrocytes. Levels of increased neurofibromin, correlated with elevated levels of activated p21Ras measured in both the fresh tumor specimens and the primary cell lines. Furthermore, when levels of activated p21-Ras were decreased in astrocytoma cells expressing the Ras inhibitory Asn 17 dominant-negative mutant, levels of neurofibromin were dramatically decreased in these cells. In addition, fibroblasts induced to express the p21ras $^{\text {val } 12}$ activating mutant, had increased concomitant expression of NF1. These results suggests that neurofibromin expression is increased in human astrocytic tumors, as a result of positive feedback regulation by increased levels of activated p2 IRas.

\section{P.081}

Mutant Epidermal Growth Factor Receptors (EGF-R) in Glioblastoma Multiforme Operative Specimens:

\section{P.K. Lala, N. LaU, M. FeldKamp, A. GuHa (Toronto, Ontario)}

Glioblastoma multiforme (GBM) is the most common type of primary brain tumour in adults. A number of genetic events may lead to the formation of GBMs, including amplification of EGF-Rs in about $50 \%$ of GBMs. The product of this gene is a $170-\mathrm{kDa}$ glycoprotein that is expressed on the surface of many cell types. The 1186-residue protein possesses an extracellular domain that can bind ligands such as EGF and transforming growth factor-a (TGF- mutant a), a single transmembrane domain, and a $\mathrm{C}$-terminal region that has intrinsic tyrosine kinase activity. In many cases, GBMs express mutated versions of the EGF-R protein. Of these, the most common mutation is one in which a large portion of the extracellular domain (residues 6-273) is missing due to an 801-base pair deletion in the EGFR gene; it has been found to occur in $2540 \%$ of all GBMs. This $140-\mathrm{kDa}$ mutant receptor has a number of characteristic properties; first it is unable to bind ligands due to the deletion of the binding domain. Second, a novel glycine residue is introduced at the splice site, a feature which has been utilized to raise antibodies specific for the mutant receptor. Third, the receptor appears to be phosphorylated independently of ligand 
interaction, which implies a constitutively active state. Transfection of the mutant EGF-R into established glioma cell lines enhances tumour growth in vivo, but not in vitro. The current study examines the activation state of EGF-R in a cohort of 8 flash frozen GBM operative specimens. Immunoprecipitations and Western blot analysis indicates the expression of constitutively phosphorylated $140-\mathrm{kDa}$ EGF-R, in $3 / 8 \mathrm{GBM}$ specimens. Further examination will include immunohistochemical staining of cryosections of these same specimens using a monoclonal antibody that recognizes activated EGF-R. Also of interest is the signal transduction pathways utilized by the mutant EGF-R. Our lab has demonstrated that activated Ras-GTP is necessary for the proliferation of astrocytoma cell lines bearing the normal EGF$R$. The transfection of Ras-Asn 17 dominant inhibitory mutants into astrocytoma cell lines inhibits their growth, suggesting the involvement of the Ras pathway. We are examining whether the Ras pathway is also utilized by the EGF-R to send its mitogenic signals. To examine this possibility, cryosections of the $8 \mathrm{GBMs}$ are being probed with an antibody specific for activated MAPKinase, a downstream effector of the Ras pathway.

\section{P-082}

\section{Ras-GTP levels are Elevated in Human NF1 Peripheral Nerve Tumors}

A. Guha, N. Lau, D. Gutmann, A. Pawson (Toronto, Ontario), G. Boss (St. Louis, Missouri)

Neurofibromin, the protein encoded by the NF1 gene is a Ras-GAP, loss of which would lead to elevated levels of activated Ras-GTP, leading to tumor proliferation. ${ }^{32} \mathrm{P}$ Ras loading experiments in NFI sarcoma cell lines support this hypothesis, however, the status in actual tumors remains unknown. A nonradioactive quantitative colorimetric luciferase based assay for Ras-GTP using NDP kinase, was adapted in this study for tissues. Flash frozen operative specimens were homogenized and i.p. with the Ras Y13-259 antibody. Levels of GDP and GTP bound Ras were measured using the assay, and standardized for protein or DNA content of the specimens.

Two NFI neurogenic sarcomas (5 measurements) had the highest levels of activated Ras-GTP (46.0 +/-7.0 f.molGTP/mg DNA), followed by the four NFI neurofibromas (13.3+/-2.6 f.molGTP/mg DNA). These values can be compared to four non NFI schwannomas, with Ras-GTP levels of $3.5+/-.6$ f.molGTP/mg DNA. The level of NF1 expression was markedly decreased in the NFI neurogenic sarcoma by RT-PCR. Abundant type 1 and especially type 2 $\mathrm{NFl}$ isoforms were expressed by both NFI neurofibromas and non NFI schwannomas. Neurofibromin expression by immunohistochemistry was absent in the NF1 neurogenic sarcoma, but strongly positive in non NFl schwannomas. Some cells were positive while others were negative in NFI neurofibromas, reflecting the heterogenous cellular makeup of these tumors. This study introduces a technique to measure the activation status of Ras in tissues, which can be used in a variety of normal and pathological states where Ras is involved. In NFl peripheral nerve tumors levels of activated Ras are elevated, suggesting that inhibitors of Ras may be therapeutically useful in these tumors.

\section{P.083}

An In-Vivo Investigation of Brain Tumour Growth Characteristics in an Animal Model Using CT

L.L. Poczok, T.Y. Lee, R.F. Del Maestro, J. Taylor, I.A. Cunningham, D. Ramsay, J.J. Battista, B. Fisher (London, Ontario)

Background: Studies on the effect of Stereotactic Radiosurgery on the vascular parameters: Cerebral Blood Volume (CBV) and Blood Brain Barrier permeability (K), are relatively sparse. Before beginning to look at these effects, the characteristics of tumour growth must be characterized. Using a tumour model developed by Brem et al (Am J Path 131:361372,1988 ), we used a dynamic CT scanning technique to monitor the changes in $\mathrm{CBV}$, and $\mathrm{K}$ as the tumour grows.

Methods: VX2 Carcinoma was injected into the right parietal lobe of twenty, male, $3 \mathrm{~kg}$ New Zealand White rabbits. Dynamic CT studies began at 7 days post implantation and continued every 2 days until the animal was sacrificed at either $8,10,12$, or 15 days post-implantation.

Results: Table 1 shows the mean values for $\mathrm{K}$ and $\mathrm{CBV}$ at 7 , 9,11 , and 14 days after induction of the tumour.

\begin{tabular}{|lllll|}
\hline & Day 7 & Day 9 & Day 11 & Day 14 \\
\hline $\mathrm{CBV} \mathrm{ml} / \mathrm{g}$ & 0.067 & 0.125 & 0.203 & 0.208 \\
& \pm 0.025 & \pm 0.100 & \pm 0.096 & \pm 0.070 \\
\hline $\mathrm{K} \mathrm{ml} / \mathrm{min} / \mathrm{g}$ & 0.015 & 0.067 & 0.083 & 0.204 \\
& \pm 0.017 & \pm 0.076 & \pm 0.071 & \pm 0.105 \\
\hline
\end{tabular}

Conclusions: $\mathrm{CBV}$ and $\mathrm{K}$ increase with tumour growth. With this study the characteristics of the tumour model have been characterized to allow studies investigating the effect of SR on VX2 carcinoma to proceed.

\section{P-084}

\section{The Effect of Gd-DTPA on H1 Spectroscopy of In Vivo Brain Tumors}

A. Al-Shayji, A. Damyanovich, E.T. Kiriakopoulos, A. Guha, D.J. MIKulis (Toronto, Ontario)

Purpose: To determine if Gd-DPTA alters proton spectra obtained from in vivo brain tumors.

Methods: Single voxel proton spectra were obtained from six neoplasms and six normal controls before, and immediately after the I.V. administration of $10 \mathrm{cc}$ of Gd-DTPA (Magnevist - Berlex Canada). Patients for this study were selected based on the need for a gadolinium enhanced MRI scan for pre-operative assessment. The spectra were obtained on a 1.5 clinical scanner using a STEAM pulse sequence, $\mathrm{TE}=20 \mathrm{~ms}, \mathrm{TR}=2 \mathrm{sec}$. Spectral processing consisted of automatic phasing and residual water peak deconvolution using the water reference, FFT with Gaussian resolution enhancement, followed by a Marquardt fit of the most important resonances (NAA, CHO, CRE, MYO and LAC/Lipid-CH2). Peak amplitides, areas, chemical shifts and full-width-half-maxima were all calculated and compared pre- and post-gadolinium.

Results: Various effects were seen in the spectra obtained 
after gadolinium administration including line broadening and reduction in peak height of the MR visible metabolites. However, the presence of gadolinium enhancement seen on the conventional images did not predict these effects nor was there a predictable effect based on surgical pathology.

Conclusions: Gadolinium can influence MR spectra of brain neoplasm but the effects do not seem to increase the specificity of the MR spectra.

\section{P.085}

\section{Neuronavigation with the "Wand"}

\section{A. Salloum, P.B.C. Gorman, G.R.C. Quartey (Moncton, New Brunswick)}

The viewing wand is a computerized neuro navigational tool designed and manufactured by ISG Technologies, Mississauga, Ontario. The authors reports its use in a community hospital setting where it appeared very useful to provide craniotomy landmarks for excision of small brain tumors and accurate localization in cranial sinuses and skull base surgery. The additional time spent in setting up the system and registration was offset by the shorter operating time due to smaller and more accurately positioned bone flaps. The authors describe a minor transformation of the "probe" allowing frameless stereotaxic biopsies of cerebral lesions using an existing stereotaxic biopsy kit.

\section{P.086}

Multiple Sclerosis Mimicking Central Nervous System Neoplasms: Ten Cases and Recommendations Regarding Avoidance of Management Pitfalls

\section{B.D. ToYoTA (Vancouver, British Columbia)}

Multiple Sclerosis (M.S.) presents in a number of characteristic fashions that are generally well-recognized. However, despite advances in diagnostic technology, it is also well known to present diagnostic challenges, often imitating a wide variety of other neurologic pathologies.

We present ten cases which were initially diagnosed as central nervous system neoplasms based on clinical and imaging criteria, only to be proven to be demyelinating lesions associated with Multiple Sclerosis. In some cases the true diagnosis was established by lingering clinical suspicion followed by traditional means of M.S. diagnosis. However in other circumstances the patient went on to surgical exploration, including biopsy, only to reveal pathologic evidence of demyelination compatible with Multiple Sclerosis. Surgical exploration of these latter patients included significant complications related to the procedure. Upon review of these cases it is apparent that the newer diagnostic technology presents a "two-edged sword"; leading to both misdiagnosis of neoplasia as well as providing credence to a suspicion of Multiple Sclerosis.

This presentation will describe these ten patients in detail with a critical review of the diagnostic criteria that was used to arrive at the misdiagnosis of neoplasia. Additionally it will make recommendations that will help avoid similar pitfalls in the treatment of the M.S. patient who presents atypically.

\section{P-087}

\section{Sublabial Transphenoidal Surgery for Pituitary Disease}

\section{A. Malquez, H.H. ZaYAS (Havana City, Cuba)}

A series of 264 sublabial transphenoidal surgeries, performed between November 1991 and January 1995, is presented. Pre and postoperative clincal, neurophthalmogical, hormonal and radiological findings were analyzed in order to state precisely their behavior with this surgery, and its complications. There were 123 microadenomas, 73 macroadenomas, 56 tempty sella syndromes, \pm 3 microadenomas/empty sella, 4 craniopharynqiomas, 2 meninqiomas, 2 astrocytomas, 1 intracavernous carotid aneurysm. Clinical neurophthalmogical, hormonal, radiological and surgical findings are discussed. Headache was the most frequent sympton without a regular pattern and was an important complaint $(71.4 \%)$ in cases with \pm Empty Sella Syndrome \pm during the first 48 hours postoperatively, probably due to a intrasellar packing. Visual field reduction, even without becoming: tubular was an interesting preoperative finding in empty sella syndrome, improving in $76.3 \%$ cases. Prolactin levels were lower in microprolactinomas than in bigger tumors. CT scan had $90.8 \%$ accuracy. During surgery, downward herniation of the relaxed diaphragm appeared in $20 \%$ of macroadenomas, requesting a \pm proplolactic arachnoidopoxy \pm . Postoperative Diabetes Insipidue was a real problem in our series $(22.2 \%$ of microadenomas), the most frequent complication, being transient in the majority of cases. This surgery has been made in our department for the last 13 years, with an annual average of 80 cases.

\section{P-088}

\section{Lateral Sphenoid Wing Cranio-orbital Meningiomas}

H. Bader, F.A. Durity, J. Rootman (Vancouver, British Columbia)

Background: Indications and extent of surgical removal of uncommon lateral sphenoid wing cranio-orbital meningiomas is controversial. To address these issues we reviewed our experience with these lesions.

Methods: 15 consecutive patients operated between 1984-94 were reviewed. Mean age was 48 . There were 13 females and 2 males. Proptosis was present in 13, ocular symptoms in 9. CT scan, $100 \%$ revealed the extent of tumor to the orbit and sphenoid wing in all, cavernous sinus and intradural in 6 each. Mean follow up was 6 years (range $11-148 \mathrm{mo}$ ). Cranio-orbital resection was done in all and orbital reconstruction in 14. Indications for surgery were proptosis in 12 and visual deterioration in 3 .

Results: 5 had total removal, 10 aggressive subtotal removal. The main limitation for surgery was cavernous sinus involvement with minimal cranial neuropathies. All patients returned to full activities, proptosis was relieved in all 12 and vision 
improved in the 3 where it was the main indication for surgery. Complications included mild transient extra-ocular muscle paresis in 8, transient CSF leak 2, exophthalmus 1, and visual deterioration 1. None had recurrence. 3 underwent postop radiotherapy.

Conclusions: Aggressive but limited removal with no mortality and low morbidity provides long term control.

\section{P-089}

\section{Surgical Approaches to Lesions of the Lateral Ventricles}

\section{S. Brien, G. Mohr, S. Albrecht (Montreal, Quebec)}

Background and Purpose: Lesions of the lateral ventricle are often slow growing yet, they can present with sudden neurological symptoms secondary to hydrocephalus. Tumor location and ventricular topography are critical considerations in the selection of an appropriate surgical corridor.

Methods: Eight cases of lateral ventricle tumors were treated by the neurosurgical team at the Jewish General Hospital during 1994 - 1995. The tumors were located in the following areas of the lateral ventricle: three in the frontal horn, one in the body, three in the trigone, and one in the temporal horn. Three patients required pre-operative shunts. Transcallosal, transcortical transventricular, endoscopic and combined approaches were utilized. The pathologies of the lesions included meningioma, glioma, metastasis, ependymoma, central neurocytoma, and neuroepithelial cyst.

Results: All patients underwent surgical procedure(s), for diagnosis and / or tumor removal. Morbidity included one patient with a transient speech disturbance and two patients with visual field defects (unchanged from pre-op). Six patients had improvement of their symptoms. One patient expired two months post-operative due to tumor progression.

Conclusions: Although uncommon, lesions of the lateral ventricles represent a challenging technical problem. Careful pre-operative surgical planning using minimally invasive techniques is essential to limit morbidity.

\section{P-090}

Calcified Anterior Third Ventricular Meningioma - Case Report and Review of the Literature

F. Maroun, F. Costello, J.C. Jacob, M. Mangan, G. Murray, G. MATHIESON (St. John's, New Foundland)

Intraventricular meningiomas are quite rare accounting for approximately $0.7 \%$ of total occurrences. Anterior third ventricle location represent a small subset of this group and are thought to arise from the choroid plexus, telachoroida or velum interpositum.

A case of anterior third ventricular meningioma is presented. Microsurgical removal through the transcallosal approach was carried out. Post operative sleepiness for a few weeks was thought to be due to interference with deep venous drainage. Follow-up 21/2 years later showed no evidence of any clinical or neuropsychological deficit. Besides CT and MRI we feel that preoperative angiography remains essential to outline both cortical and deep venous anatomy in the management of these rare lesions.

\section{P-091}

\section{Primary Intramedullary Primitive Neuroectodermal Tumour of the Spinal Cord}

G. Skaf, S. Deme, LC. Ang, P. Cooper and D. Rowed (Toronto, Ontario)

Background: Primary primitive neuroectodermal Tumor (PNET) of the spinal cord is a rare entity.

Methods: We reported a case of a 22 year-old female with a recurrent low back pain, bilateral leg weakness and loss of proprioception, sensory level at $\mathrm{Ll}$, saddle anesthesia, urinary incontinence and decreased rectal tone. Computed Tomography (CT) myelogram revealed an intramedullary mass that caused a total block at T12-L1 level.

Results: The patient underwent a gross total removal of an intramedullary tumour. Pathology findings were consistent with small round cells with hyperchromatic nuclei, high mitotic figures and some neuronal and glial differentiation. Electron microscopy confirmed the diagnosis of PNET. Magnetic resonnance imaging (MRI) of the cranio-spinal axis showed no evidence of an intracranial tumor. Radiation therapy was postponed because of wound infection. She was treated with antibiotics and six weeks later was readmitted for worsening neurological condition. A spinal MRI showed local recurrence. Chemotherapy was started awaiting wound healing.

Conclusion: Primary spinal PNET is rare entity with predilection to the cauda equina but its occurrence above that level is even rarer. The possibility of drop metastasis from medulloblastoma has to be excluded. This tumour has a very poor prognosis.

\section{P.092}

A Remote Sensing Tool for the Measurements of Patient Position Monitoring During Stereotactic Radiation Therapy

L.H. Gerig, S. El-Hakim, J. Szanto, S. Malone, D.S. Salhani, T. YaychuK, A GiRard (Ottawa, Ontario)

Geometric accuracy of dose delivery is a critical component of stereotactic radiation therapy (SRT). We have developed an optically based system for real time monitoring of patient position which measures and reports variations in patient position every 5 seconds.

The system measures intra (setup) and inter daily treatment displacements and allows verification prior to beam on. System hardware consists of $2 \mathrm{CCD}$ cameras focused on the treatment unit isocentre. Passive reflective targets are placed on the patient surface and the SRT frame and are illuminated by very low power diffuse $810 \mathrm{~nm}$ light. $810 \mathrm{~nm}$ band pass filters on each camera lens help provide contrast between the targets and patient. The images are digitized and processed on a PC where our 3D vision software determines the spatial coordinates of each target using a triangulation algorithm.

Our tests indicate an accuracy and reproducibility of better 
than $0.2 \mathrm{~mm}$ (absolute) over extended periods ( $>1$ month). Clinically we have followed several SRT patients as reported in our companion paper.

This communication provides technical details of the system and a detailed analysis of its performance in the laboratory and the clinic.

\section{P-093}

The Clinical Use of a Patient Position Monitoring System as a Verification Tool for Fractionated Stereotactic Radiotherapy

S. Malone, L. Gerig, J. Szanto, A. Girard, S. El-Hakim, H. RaGnITZ (Ottawa Ontario)

In Fractionated Stereotactic Radiotherapy (SRT) it is critical to have a system that is accurate, reproducible and verifiable. A Patient Position Monitoring System (PPM) was developed as a QA and verification tool integral to our SRT program. PPM is now routinely used for inter and intra fraction patient position verification.

The SRT verification system involves a multi step process, the final step being PPM. PPM is an optically based system which measures retro reflective targets located on the stereotactic frame and patient's forehead. Two ceiling mounted CCD cameras recognize and detect the targets. The target position is calculated using a triangulation algorithm. The setup measurement takes seconds giving a rapid verification of the isocentre. PPM also monitors patient position every 5 seconds during and individual treatment.

PPM is used to verify the isocentre prior to delivery of SRT. The mean displacement of the isocentre between fractions is 0.3 $\mathrm{mm}$ (range $+/-0.8 \mathrm{~mm}$ ). During a given fraction the maximum patient motion recorded by PPM is less than $1.5 \mathrm{~mm}$.

PPM is a unique tool for rapid verification of the isocentre and for monitoring patient position during SRT. The Ottawa system for SRT delivery is accurate and reproducible.

\section{P-094}

\section{A Fractionated Stereotactic Radiotherapy Delivery System}

J. Szanto, L.H. Gerig, S. Malone, A. Girard, H. Ragnitz, S. El-Hakim (Ottawa, Ontario)

In Ottawa we have developed a system for fractionated stereotactic radiotherapy treatment of various brain malignancies.

Patients are immobilized in a very accurate, stable, reproducible, noninvasive, relocatable stereotactic frame which is based on a custom made Cobalt-Chrome bite-block that locks into the undercuts of the teeth.

Verification of positioning:

a) CT verification: Following treatment planning the calculated Cartesian co-ordinates of the planned isocentre are marked on the localizer frame. The isocentre verified by obtaining a CT slice through the plane of the markers.

b) Check film: Patient set-up is verified using radio-opaque markers placed on the patient's skin. AP and lateral double exposed check films are then taken and the projected distances between the isocentre and the skin markers are measured and verified.

c) PPMS: Our optically based Patient Position Monitoring system (PPM) is used to measure set-up and treatment deviations, sampling the patient position every 5 seconds. The PPM system is described in a companion paper.

d) In this paper we will present technical details of the system.

e) Spatial accuracy of the treatment will also be discussed.

\section{P-095}

New Windows-based Software for Stereotactic Biopsy and Radiosurgery Planning

B. TOMPa, A. Nicholson, R. Ramani, M.L. SchwartZ (Toronto, Ontario)

Our centre has been involved in radiosurgery since 1988 , using software originally obtained from CMI of Montreal. Like that original software, the new system has very modest computing requirements, that is, a personal computer (PC). Currently, General Electric CT and MRI images are imported to the program via a hospital-wide electronic network. The DICOM standard will be incorporated when our hospital's scanners support it. This will allow easy retrieval of images from all compliant sources via an integrated image retrieval and planning interface. At present, multiple scan, reconstructed scan or digital subtraction angiography images may be displayed simultaneously with a synchronized and dynamic cursor indicating real-space coordinates over different views. Any plane reconstructed view of a scan image chosen by the user may be displayed. For any individual displayed image, user-selected contrast and windowing are available. Any cartesian co-ordinate user-definable stereotactic frame may be specified.

For radiosurgery, volume-oriented dose calculation and storage are provided. Interactive target localization and planning permits the easy addition of multiple isocentres.

For users familiar with the Windows interface, use of the program is rapidly and intuitively learned. As the features of the program are still undergoing metamorphosis, collaboration with clinicians in other centres is sought.

\section{P.096}

Frequency of Follow-up MRI Scanning in Patients with Malignant Glioma - Will Detecting Asymptomatic Recurrence Benefit our Patients?

\section{FULTON (Edmonton, Alberta)}

Background: Follow-up of patients with primary malignant brain tumor, intended to identify response, recurrence and side effects of therapy, involves repeated neuro exams and CT or MRI. The neuro exam alone does not exclude recurrence. Detection of asymptomatic recurrence allows therapy before neurological deterioration and may improve quality of life. It is not known whether or not detection and treatment of asymptomatic recurrence prolongs time to tumor progression (TTP) or 
survival time (ST). Recent budget cuts have resulted in the need to reduce the frequency of routine follow-up scans in patients with brain tumors.

Methods: We reviewed the medical records of 89 consecutive patients treated with chemotherapy plus or minus surgery for their first recurrence of supratentorial malignant glioma. Initial treatment consisted of surgery followed by radiation therapy \pm adjuvant chemotherapy. Initially, patients routinely had CT or MRI scans scheduled prior to each dose of chemotherapy (6 to 8 week interval). Later, because of budget cuts, scans were scheduled prior to alternate doses of chemotherapy (12 to 16 week interval). Scans were done early for signs or symptoms of recurrence.

Results: Median TTP (MTTP) in weeks from date of progression was 20.9 for all patients, 30.8 for those asymptomatic at the time of recurrence and 16.3 for symptomatic patients. $(p<0.05)$ Median ST (MST) in weeks was 46.1 for all patients, 68.0 for those asymptomatic patients and 35.5 for symptomatic patients. $(p<0.05)$ Median TTP (MTTP) in weeks from date of progression was 16.3 for patients with glioblastoma multiforme, 20.2 for asymptomatic patients and $\mathbf{1 5 . 1}$ for symptomatic patients. $(\mathrm{p}<0.05)$ Median ST (MST) in weeks was 32.3 for all patients, 39.9 for asymptomatic patients and 23.8 for symptomatic patients. $(\mathrm{p}<0.05)$

Conclusions: Differences in MTTP and MST are significant but may still be accounted for by lead time bias. Detecting asymptomatic recurrence may be beneficial but was not increased by more frequent scanning in this study.

\section{P-097}

\section{Steroid Toxicity in Brain Tumor Patients (BTP)}

\section{B.M. ChaudhuRI (Montreal, Quebec)}

Background: Since GALICICH and FRENCH determined that dexamethasone (DEX) could be used in reducing the mortality in BTP in the early 1960's, the standard protocol of treating peritumoral cerebral edema has been $4 \mathrm{mg}$ of DEX q6h. However, no one has looked at comparing DEX against any weaker glucocorticoids (GC), presumably because of their greater mineralocorticoid effects. This poster investigates the side effects of DEX, its mechanism of action, and examines the possibilities of other treatments for peritumoral cerebral edema.

Methods: An extensive literature review was undertaken.

Results: DEX has a greater propensity to induce morbidity than weaker GC-like methyl-prednisolone or prednisone. DEX is a fluorinated glucocorticoid. Side effects may be divided into three groups: mineralocorticoid effects, the withdrawal of the drug or chronic excess GC administration leading common problems, e.g. opportunistic infections and steroid myopathy. Other treatments have been looked at, such as nonsteroidal antiinflammatory agents (NSAIDS) etc., without success.

Conclusion: A clinically randomized control trial has never been done showing that DEX is any more effective than other GC in the treatment of BTP. This trial would be advisable to decrease the morbidity of BTP. Further research is needed into the management of peritumoral cerebral edema.

\section{SESSION 2}

\section{Cerebrovascular Disease}

\section{P-098}

\section{Isolated Angiitis of the Central Nervous System}

D. DeRubeIS, J. SidDiQI, (London, Ontario)

Background: Isolated angiitis of the central nervous system is an uncommon clinicopathologic entity characterized by vasculitis restricted to the vessels of the CNS without apparent system manifestations of vasculitis. Recurrent cerebral infarction leading to death within a few years is the usual outcome. Aggressive immunosuppression may alter this course.

Methods and Results: We report a case of a 45-year old man presenting with sudden severe suboccipital headache and left hemianopia. MRI revealed bilateral occipital infarcts. Angiography showed multiple small arterial branch narrowing and beading. Immune markers for systemic vasculitis were negative and LP was unremarkable. The diagnosis of isolated cerebral angiitis was made and treatment with IV steroids with the aim of immunosuppression was undertaken. Symptomatic improvement occurred within 48 hours of treatment.

Conclusions: This is an uncommon presentation of a rare condition. Previous cases from our institution and the literature are reviewed.

\section{P-099}

\section{Cerebral Vasculitis: Relationship with Cocaine Abuse}

P. CossetTe, R. LABRecQue (Montréal, Québec)

Objective: To report a case of isolated cerebral vasculitis in a chronic cocaine user.

Background: Cocaine abuse has been associated with hemorrhagic and ischemic strokes in young adults. However, the pathophysiology of these strokes is not clearly understood. Cerebral vasculitis has been reported in rare cases on the basis of angiogram ( 3 patients) or by cerebral biopsy (4 patients).

Methods: A 27 year-old man with a history of chronic cocaine abuse presented with acute headache and right hemiparesis 3 weeks after his last acknowleged usage. On the eighth hospitalisation day, the patient had second attack of acute headache associated with quadraparesis and pseudobulbar palsy. His head MRI showed bilateral T2-enhanced lesions in the midbrain consistent with acute strokes. The angiogram revealed multifocal segmental stenosis and beading strongly suggestive of cerebral vasculitis. After a 2 week interval, a control angiogram showed progression of the vasculitic lesions. A slight protein elevation was found at the CSF examination. There was no evidence of systemic vasculitis nor of other etiology for stroke. The patient was treated with nimodipine and corticosteroids. 
Conclusion: This case and other reports suggest that vasculitis may be related to cocaine abuse. However, a primary cerebral angiitis can not be completely excluded.

\section{P-100}

MELAS Syndrome in a 45-year Old Male with New Onset Stroke and Seizures

P. Mandalfino, T. Rupar, R. Hammond, D. Moulin (London, Ontario)

Background: Neurological syndromes associated with mitochondrial myopathies are recently described entities with variable presentations including common neurological illnesses such as stroke. As clinical awareness has increased, patients are being identified at younger ages. However, the diagnosis should be considered in the older patient without other identifiable causes, especially with a background of other neurological manifestations of a mitochondrial disorder.

Methods: We describe a 45-year old man with sudden onset aphasia and painful rightsided sensory seizures on a background of progressive neurosensory hearing loss since his teens. Serum lactate was elevated and a CT scan of the head showed basal ganglia calcifications consistent with a mitochondrial disorder. Muscle biopsy was obtained.

Results: Gomori trichrome showed ragged red fibres and histochemical staining with NADH and SDH confirmed this finding. Electron microscopy showed subsarcolemmal accumulations of abnormal mitochondria confirming the diagnosis of a mitochondrial myopathy. Analysis of muscle mitochondrial DNA identified an $A$ to $G$ point mutation at nt 3243 characteristic of MELAS syndrome. The mutation was absent in leucocytes. We are currently testing other potentially affected family members.

Conclusion: Mitochondrial myopathy needs to be kept in the differential diagnosis of common neurological problems such as stroke and seizures. Diagnosis of this maternally inherited disorder has significant implications for the patient and for family members at risk who may require genetic counselling.

\section{P-101}

\section{Adult Onset Leigh's Disease}

F. Tanha, C.W. McCormick, R.A. Purdy, V. Sangalang, Y. SEGEv-SHNAPICK (Halifax, NS.)

Introduction: Leigh's disease or Subacute Necrotizing Encephalo-myelopathy is a rare metabolic disease usually presenting in the pediatric age group. We present an autopsy proven adult onset case with coexisting Gilbert's syndrome.

Case: A 54-year-old Caucasian male presented with subacute onset of subcortical aphasia and bilateral thalamic lesions, visualized on cranial CT and MRI, with rapid progression and extension of lesions associated with neurological deterioration. He was found to have Gilbert's syndrome. Stereotactic thalamic biopsy revealed extensive focal acute hemorrhagic necrosis with prominent axonal injury. Treatment with IV acyclovir, thiamine, and dexamethasone was ineffective. He died three weeks after the onset of symptoms. Autopsy revealed extensive acute hemorrhagic necrotizing lesions involving diencephalon, striatum, midbrain, pons, medulla, and hippocampus, with extension of hemorrhage into the fourth ventricle. Electron microscopy showed accumulation of abnormally structured mitochondria in swollen axons.

Discussion: Radiologic and neuropathologic findings were consistent with Leigh's disease. It has been found to be due to a deficiency of several enzymes necessary for oxidative metabolism. Of clinical interest is thalamic aphasia with radiological correlation. Also interesting is the association of Leigh's disease and Gilbert's syndrome. It is possible that a similar enzymatic deficiency is responsible for both disorders; if true, this would put a new perspective on Gilbert's syndrome which is thought to be a benign disorder. Our enzymatic studies of this patient are pending.

\section{P-102}

Spontaneous Cerebral Hemorrhage as Manifestation of Sipple Syndrome

\section{P. GRammond, M. Thibault (Québec, Québec)}

Background: Hemorrhagic stroke has occasionally been related to transient hypertension such as sympathicomimetic drug intake, eclampsia and pheochromocytoma. A 35 year old male presented with a left lobar cerebral hemorrhage as the first manifestation of multiple endocrine neoplasia type IIA (Sipple syndrome).

Case report: The patient had a familial history of Sipple syndrome and presented an acute, partial Broca aphasia and right upper limb weakness. He has also had a severe head injury with residual rubral tremor, ataxia and controled epilepsy. Biological findings were normal except : calcitonin : $1725 \mathrm{ng} / \mathrm{l}$ (normal < 100 ) and the 24 hour urine sample with increased epinephrine $900 \mathrm{nmol} /$ day (normal < 110 ), norepinephrine $1260 \mathrm{nmol} /$ day (normal < 470) and vanillylmandelic acid $64 \mathrm{mmol} /$ day (normal 2-60). Cerebral CT scan demonstrated a left posterior frontal hemorrhage and encephalomalacia in the left frontal lobe. Cerebral angiography and cardiac echography were normal. Abdominal MRI revealed a cystic lesion of the right adrenal gland and hyperplasia of the left. Thyroid echography showed solid, nodular heterogeneous lesions. The pathological diagnosis was bilateral pheochromocytomas and medullary carcinoma of the thyroid gland.

Conclusion: Cerebral bleeding in patients with Sipple syndrome has rarely been reported. Few autopsies of patients with major cerebral hemorrhage have revealed pheochromocytoma. Spontaneous cerebral hemorrhage is an exceptional initial manifestation of Sipple syndrome, probably related to hypertensive crisis. 


\section{P-103}

\section{Visual Pathways Compression by a Giant Fusiform Basilar} Aneurysm

\section{G. SKAF, L.C. ANG (Toronto, Ontario)}

Background: Visual deterioration due to giant basilar aneurysm is very rare.

Methods: We report a case of a 58 year-old man with a previous history of left hemispheric cerebrovascular accident, who was found to have a dolichoectasia of the basilar artery that progressed to aneurysmal proportion and bled three years later.

Results: He was comatose for two months but recovered gradually and was left with a right facial and left sixth nerve palsy. Subsequently he reported a progressive deterioration of his visual status without computed tomography (CT) findings except for increased aneurysmal size. His visual acuity dropped to 20/80 OD, visual field showed a loss of the right hemianopic fields (more severe on the left) and his left optic disc was pale. Three years after the bleed, he expired. The neuropathologic examination shows that the fusiform aneurysm $(7 \times 5 \times 2 \mathrm{~cm})$ is compressing the optic chiasm, the brainstem and medial temporal lobes. There is evidence of hemorrhage and patchy demyelination of both optic nerves, the optic chiasm and the optic radiations, more severe on the left.

Conclusion: We suggest that the degenerative changes in the optic pathways which account for the patient's visual field defect and the progression of his visual symptoms are the result of compression by the giant aneurysm.

\section{P-104}

\section{Subarachnoid Haemorrhage in a Canadian Community Hospital}

W.B. Wheelock, N.A. Russell, J.Arditti, J. Kenney, G.M. RuSSELl (Saint John, New Brunswick)

Background: This study is a data analysis of the presentation and management of patients with subarachnoid haemorrhage and aneurysm treated in a Canadian Community Hospital.

Methods: Patients admitted to the Saint John Regional Health Center, during the years 1992-94, with a diagnosis of subarachnoid haemorrhage or non ruptured aneurysms were identified. Their records were reviewed for details of clinical presentation, management and outcome.

Results: There were 87 such patients. 68 had a ruptured aneurysm, 10 had no demonstrable cause for the haemorrhage and 9 had "incidental" aneurysms. The age range was 23 to 81 years. They were also subdivided into three age related groups. $19(22 \%)$ were under 40 years, $34(39.1 \%)$ were between 40 and 59 years, and $34(39.1 \%)$ were aged 60 or above. Overall there were 57 females $(65 \%)$ and 30 males $(35 \%)$. Ratio is $2: 1$. The age groups were further subdivided according to sex. Data concerning aneurysm location, rebleeding, and vasospasm, as well as the management outcome are presented.

Conclusion: The outcome for these patients compares favorably with results currently reported from larger institutions.

\section{P-105}

Polycystic Kidney Disease Associated With Asymptomatic Choroid Plexus Cysts

\section{J.N. ScotT, G. KleIn, C.H. Dyke (Calgary, Alberta)}

Background: Patients with polycystic kidney disease tend to have other congenital anomalies, including intracerebral berry aneurysms and asymptomatic extrarenal cysts within the liver, spleen, pancreas, and lungs. There are no reports of polycystic kidney disease associated with cysts of the choroid plexus.

Methods: Case study.

Results: A 64-year-old woman with polycystic kidney disease and asymptomatic hepatic cysts was seen in the Stroke Prevention Clinic in Calgary, Alberta for risk assessment. The patient had mild hypertension and a first-degree relative with polycystic kidney disease, who died of complications from subarachnoid hemorrhage caused by cerebral aneurysm. In our patient, while no aneurysm was seen, the diagnosis of numerous bilateral choroid plexus cysts was made by magnetic resonance imaging (MRI).

Conclusion: We describe a patient with polycystic kidney disease and multiple cysts of the choroid plexus, and suggest that an as yet unreported association may exist between these two conditions.

\section{P-106}

\section{Cerebral Venous Thrombosis: a Series of Eight Cases}

S. Meckling, O. Suchowersky, T. Feasby, K. Hoyte, W. MurPHY (Calgary, Alberta)

Background: In the past, cerebral venous thrombosis (CVT) was considered to be rare; treatment remains controversial. We recently reviewed our experience in this condition.

Methods: Eight cases of CVT were seen in the 2 major teaching hospitals in Calgary over the past 18 month period. Cases were reviewed with respect to clinical information and treatment outcome.

Results: Of the eight cases, 7 were female and 1 was male. Age range: 15 - 50 yrs. Clinical presentation: severe headache (8pts), seizures (5 pts), focal neurological signs (4 pts). One patient had headache symptoms indistinguishable from migraine; one patient presented with symptoms of subarachnoid hemorrhage. Diagnosis was made on CT scan in only 3 patients. MRI and/or angiography was required in the rest. Etiological associations: oral contraception (3 pts), post-partum (2 pts), intra-partum (1 pt), postsurgical (1 pt), protein $\mathrm{C}$ deficiency ( $1 \mathrm{pt})$. One patient, who had a large associated hemorrhage, died. Treatment in most consisted of 10 days of IV heparin, followed by $3-6$ months of coumadin.

\section{P-107}

Fibrinogen as a Marker of High Grade Carotid Stenosis and Cerebrovascular Events

L. Vieira, C. Wolfson, R.Coté (Montreal,Quebec)

Background: A simple clinical test to determine which 
patients with asymptomatic carotid disease are at increased risk for cerebrovascular events, could improve stroke prevention.

Objective: To measure levels of serum fibrinogen in patients with asymptomatic carotid disease and assess if they correlate with degree of stenosis and occurrence of events.

Methods: Prospective study of patients referred for asymptomatic carotid bruits or stenosis and age-matched controls. Blood was drawn for serum fibrinogen and carotid stenosis was measured by ultrasonography. Patients were followed for the occurrence of vascular events including stroke, transient ischemic attack, myocardial infarction, or vascular death.

Results: 144 patients and 59 controls were entered into the study. The average age was 68.1 years. The average length of followup was 21.7 months (range $1-37 \mathrm{~m}$ ). Serum fibrinogen level was significantly higher $(3.26 \mathrm{~g} / \mathrm{L}, \mathrm{p}=0.006)$ in patients with stenosis $\geq 50 \%$ than those with lower degrees of stenosis or controls. More vascular events occurred in patients with $\geq 50 \%$ carotid stenosis and higher fibrinogen levels than in those with $<50 \%$ stenosis $(\mathrm{p}<0.05)$.

Conclusions: Serum fibrinogen is a simple clinical test which can identify patients with asymptomatic bruits and potentially high grade carotid stenosis with increased risk for cerebrovascular events.

\section{P-108}

\section{Mechanical Prosthetic Cardiac Valve Fragments Causing Cerebral Emboli - Case Report.}

\section{D.M. WINGERCHUK AND J.R. FULGHAM (Rochester, Minnesota)}

Background: Emboli from mechanical prosthetic cardiac valves are usually platelet-fibrin thrombi, however, at least one case of cerebral embolization of actual valve fragments has been reported. Metal strut fracture and peripheral embolization have been documented with Bjork-Shiley prostheses. We report the magnetic resonance (MR) imaging appearance of multiple cerebral emboli from metallic cardiac valve fragments.

Methods: Case report.

Results: A 35 year-old female developed Streptococcus viridans prosthetic valve endocarditis. She had congenital aortic stenosis requiring multiple operations, including implantation of a Bjork-Shiley aortic prosthesis at age 20 and Medtronics-Hall aortic and mitral valves at age 24 . Neurologic examination was normal. MR imaging of the brain with T1- and T2 weighted, spin density, and T2-gradient echo sequences revealed multiple circular signal voids in all vascular territories. The lesions had regular, hyperintense borders and no gadolinium enhancement. MR angiography revealed 2 unrelated mycotic aneurysms. MR scans 11 and 42 days later were unchanged. She later died of cardiac failure; an autopsy was refused.

Conclusions: The multifocal MR imaging abnormalities are most consistent with ferromagnetic artifact, likely due to asymptomatic metallic emboli in a patient with a prior Bjork-Shiley prosthetic valve. This is the first report describing the MR appearance of this embolic complication.
P-109

\section{A Prospective Study of Crossed Cerebellar Diaschisis in Hemispheric Stroke}

D.J. Gladstone, S.E. Black, L. Ehrlich, C. Caldwell, F. LeibovitCH, C. SzEkely (North York,Ontario)

Background: Although crossed cerebellar diaschsis (CCD) is commonly observed in hemispheric stroke its clinical, anatomi$\mathrm{cal}$, and prognostic significance remains controversial and few studies have followed patients serially.

Methods: ${ }^{99 m}$ Tc-HMPAO SPECT scans were obtained in 244 stroke survivors at three time intervals post-stroke (0-7 days, 860 days, 1 year). CCD was classified by visual analysis as absent, mild, or severe and compared to lesion localization on CT. A standarized stroke scale was adminstered acutely at 1 month, 3 months, and 1 year post-stroke.

Results: CCD was present in $73 \%(178 / 244)$ of patients acutely: in $100 \%$ of those with severe stroke scores, $88 \%$ with moderate scores, and $42 \%$ with mild scores. CCD persisted for up to two months in $79 \%$ and at one year in $60 \%$. Acute CCD correlated with one year stroke score $(p<.01)$. Total stroke scores were worse in the severe CCD group compared to the absent $C C D$ group and showed improvement over time. Interestingly, this same pattern was found for motor but not language subscores. Anterior damage (basal ganglia, internal capsule, deep white matter) on CT best predicted CCD $(<.01)$.

Conclusions: Crossed cerebellar diaschisis occurs in the majority of acute hemispheric stroke patients and correlates with stroke severity both cross-sectionally and longitudinally. Anatomically, it is associated with disruption of primarily fronto-ponto-cerebellar connections.

\section{P-110}

Antithrombotics and Brain Attack: Review of 90 Patients with Acute Ischaemic Stroke at Kingston General Hospital

\section{D.B. Macdonald, F.J. Espinosa (Kingston, Ontario)}

Background: The main aim of this study was to document the current acute management of stroke patients at the Kingston General Hospital (KGH) and to determine the need and potential for new innovations in therapy.

Methods: The charts of 90 stroke patients treated acutely at $\mathrm{KGH}$ were reviewed with attention to presentation, time parameters in management, diagnostic tools, and outcome.

Results: The patients presented with a variety of symptoms, histories and clinical pictures as is expected for stroke victims. Half of stroke patients seen at KGH have been transferred from a community hospital. Forty-three percent of the patients arrived at $\mathrm{KGH}$ within $4 \mathrm{~h}$ of stroke onset, but only $13 \%$ of patients were imaged with Computed Tomography (CT) within $4 \mathrm{~h}$ of stroke onset. Forty-four percent of patients required continuing chronic care and an additional 34\% required in hospital rehabilitation. Antithrombotic therapeutics include antiplatelet therapy, anticoagulation, or supportive therapy alone. Six percent of patients treated with antiplatelets, $61 \%$ of those anticoagulated, 
and $42 \%$ of those who received supportive therapy alone had poor outcomes (chronic care, or death). Included is a case report of a thirty-six year old woman who arrived within $4 \mathrm{~h}$ and was treated with local intra-arterial thrombolytics, an aggressive option to current antithrombotic therapy.

Conclusions: More urgent stroke management and decreased delay to $\mathrm{CT}$ imaging could make trials of aggressive acute stroke therapies feasible at $\mathrm{KGH}$.

\section{EPILEPSY}

\section{P-111}

Intraventricular Recording During and After Stereotactic Amygdalohippocampectomy

\section{W.T. Blume, A.G. Parrent, M.Kaibara (London, Ontario)}

Background: Whether interictal electrocorticogram spikes correlate with seizure outcome has always been doubted and a recent study in our centre (Kanazawa et al, 1995) found no correlation between interictal electrocorticogram spikes in outcome.

Method: During sequential lesions at stereotactic amygdalohippocampectomy, mesial temporal spikes and seizures were recorded using a 5 contact intraventricular (IV) strip electrode inserted through an occipital burrhole.

Results: Although interictal spikes decreased by 65 to $86 \%$ in 4 of the 10 patients, little to no alteration in spike quantity occurred in the remainder despite sequential radiofrequency lesions of the amygdala and hippocampus constituting the procedure. This contrasts with a $90 \%$ or greater seizure reduction in 9 of 14 patients.

Conclusion: Interictal temporal electrocortical spikes, whether recorded on the temporal convexity (Kanazawa et al, 1995) or from the mesial aspect of the temporal lobe, from our intraventricular recordings, do not correlate with seizure outcome. Although interictal EEG spikes correlate well with side of principal epileptogenesis (Blume et al, 1993) no quantitative relationship between spikes and seizure outcome was demonstrated in this study. This suggests that temporal epileptogenesis operates in parallel, not series circuits.

\section{P-112}

Electrographic Changes at Ictal Onset in Subdurally Recorded Temporal Seizures Demonstrated by Frequency Analysis

\section{J.F. Lemieux, W.T. Blume, M. Kaibara (London, Ontario)}

Background: We studied the progressive frequency changes as the seizure begins and early in its course comparing involved and non-involved electrode positions.

Method: Clinically typical seizures were recorded by subdural strip electrodes, digitised at $200 \mathrm{Hertz}$, stored through a telemetry system, and visually assessed offline to distinguish involved and non-involved electrode positions. Frequency analysis was performed by FFT to display frequency modifications over time.
Results: More simultaneously occurring frequencies appeared in electrodes of origin than in uninvolved areas and the alterations of such frequencies were more numerous in such electrodes than those of later or non-involvement. Although there were several shared morphologies of frequency change between electrodes of onset with those of later involvement, there were more frequent oscillations between greater and lesser frequency complexity in seizure onset electrodes.

Conclusion: Recording electrodes detect alterations in multiple neuronal aggregates. The greater complexity and fluctuation of frequency in areas of seizure onset likely represent successive engagement of additional neuronal pools whose activity gradually becomes more synchronous (lower complexity) before additional neuronal pools are involved.

\section{P-113}

A Retrospective Study of Lamotrigine Long-term Safety and Efficacy in Adult Patients with Uncontrolled Generalized and Partial Epilepsies

F. ANDERMANn (Montreal, Quebec), J. BRUNi (Toronto, Ontario), A. Guberman (Ottawa, Ontario) on behalf of The Canadian Lamictal EDRP Study Group

Background: Lamotrigine is an anti-epileptic drug with proven clinical efficacy as add-on therapy in patients with uncontrolled generalized and partial epilepsies.

Methods: An open-label retrospective study was conducted to collect long-term safety and efficacy data from the medical records of patients treated with Lamotrigine through the Canadian Emergency Drug Release Program, from December 1991 to November 21, 1994. The study included 624 patients and data collected from the 311 adult patients are reviewed.

Results: A total of $32.5 \%$ of the adult patients with a single seizure type experienced a $\geq 50 \%$ reduction in seizures. In patients with multiple seizure types, at least one seizure type was improved ( $\geq 50 \%$ reduction in seizures) for $62.3 \%$ of the patients and all seizure types were improved for $40.6 \%$ of the patients. Complete control was obtained in $7.4 \%$ of patients. The mean total daily dosage of Lamotrigine was $255 \pm 155 \mathrm{mg}$ at the final visit.

Conclusions: There were no notable differences in the safety and efficacy data collected in this long-term study compared to the results from previous clinical trials with 2 to 3 months of therapy. Lamotrigine is an effective and safe drug used in a clinical setting for intractable seizures of various types in adults.

\section{P-114}

\section{Neurontin Evaluation in Clinical Practice}

\section{J. BRUNI, The Neon Study Group (Toronto, Ontario)}

Gabapentin (Neurontin ${ }^{\circledR}$ ) is indicated as adjunctive therapy in patients with partial seizures. The safety and efficacy of gabapentin has been established in controlled trials in patients with partial seizures refractory to various combinations of standard antiepileptic drug therapy, representing some of the most 
difficult to treat patients. The present study describes the use of gabapentin as the first add-on AED when traditional monotherapy agents fail. 130 adult patients whose complex partial seizures (with or without secondary generalization) were uncontrolled on only carbamazepine, or phenytoin, or both, were entered into a 5-month, open label multicenter trial (35 sites). Gabapentin was titrated to $1200 \mathrm{mg} /$ day over a 10 -day period, and maintained at this dose for 1 month. If adequate seizure control was not obtained, gabapentin was titrated to an effective tolerable dose (maximum $2400 \mathrm{mg} /$ day) over the remaining 4 months of study. The study was conducted under conditions that closely matched actual clinical practice. Other than gabapentin therapy, investigators were requested to follow their usual course of patient management. Patient demographics were as follows: $65 \%$ were female, $35 \%$ were male; the mean age was $41.5 \mathrm{yr}$; the median duration of epilepsy was $19.5 \mathrm{yr}$; and etiologies were as follows: unknown in $48 \%$, infection in $10 \%$, congenital and head trauma in $9 \%$, birth complications in $7 \%$, and of other origin in $17 \%$. Median seizure frequency at baseline was 3.0 seizures per month (range, 1 to 4 ). Preliminary results from completed patients suggest that gabapentin is well tolerated and is an effective first add-on antiepileptic agent. Final analysis of efficacy, safety, tolerability and effect on quality of life will be presented.

\section{P-115}

\section{Elevated Ethanolamine Levels Due to Vigabatrin (Sabril ${ }^{\circledR}$ )}

\section{J. DoOley, K. Gordon, K. DoOley, (Halifax, Nova Scotia)}

Background: Elevated ethanolamine levels on quantitative amino acid analysis are considered a marker for neurological dysfunction in children. We present 2 children who were found to have markedly elevated ethanolamine levels caused by concomitant vigabatrin therapy.

Cases: Patient 1 was a 29 month old girl who presented at 4 months of age with infantile spasms. She was treated with vigabatrin and on amino acid analysis her ethanolamine level was $10,809 \mathrm{mmol} / \mathrm{mol}$ creatinine $(\mathrm{N}=966)$. As she was on vigabatrin monotherapy, an amino acid analysis of a solution of vigabatrin was undertaken using a Beckman B6300 amino acid analyzer. Samples of the vigabatrin chromatographs showed ethanolamine peaks identical to those obtained from the patient's serum. Patient 2 was a 14 month old whose parents described both generalized tonic-clonic and simple partial seizures. Investigations, while taking vigabatrin, included a quantitative amino acid analysis which demonstrated an ethanolamine level of 1386 $\mathrm{mmol} / \mathrm{mol}$ creatinine $(\mathrm{N}=9-66)$. Video EEG monitoring showed that her abnormal episodes were not seizures and she was weaned from her vigabatrin. Repeat amino acid analysis, when off medication, showed an ethanolamine level of $<9 \mathrm{mmol} / \mathrm{mol}$ creatinine.

Conclusions: It is important to be aware that children who are taking vigabatrin may have spurious elevations of ethanolamine on amino acid analysis.

\section{P-116}

\section{Progressive Irreversible Ataxia After Long Term Phenytoin Therapy}

R. Benabou, S. Carpenter, F. Andermann, F. Dubeau, A. SHERWIN (Montreal, Quebec)

Background: Ataxia due to phenytoin intoxication is usually reversible and well known. Progressive ataxia after several decades of treatment in the absence of toxicity has not been emphasized.

Methods: Two patients with progressive ataxia, without alternative explanation, after searching investigation, are presented, including autopsy findings in one.

Results: Ataxia developed de novo in two elderly males with mild generalized epilepsy. They had received phenytoin for over three decades. At onset of ataxia, doses and levels were low. They improved slightly when the medication was replaced but remained ataxic though able to walk alone. One patient died due to complications of non malignant esophageal obstruction. $\mathrm{He}$ had cerebellar cortical atrophy.

Conclusions: Progressive ataxia unrelated to currently high levels may occur in patients receiving long term phenytoin treatment. Unlike that found in acute toxicity, this type of ataxia is permanent.

\section{P.117}

Outcome of Surgical Management in Childhood Adolescent Epilepsy

D. Keene, I. Loy-English, M. Higgins, E. Ventureyra (Ottawa, Ontario)

Objective: To assess the role of surgical intervention in the treatment of children and adolescents with epilepsy.

Method: This was a retrospective study done at the Children's Hospital of Eastern Ontario of patients who through the years of 1981 and 1994 had received surgical intervention for the management of their epilepsy. Consisted of a retrospective chart review for historical data. The present clinical status was obtained by structured telephone interview. Quality of life measurement was obtained via telephone using QOLIE.

Result: 66 patients were included in this review (41 females, 25 males). Age of onset of seizures was $5.1+4.4$ years. Surgical intervention was done at $12.0+4.8$ years of age. Follow-up interval post surgery was $7.63+3.9$ years. Area of resection was temporal lobe in 42 patients; frontal lobe 7; parietal lobe-5; frontotemporal-2; hemispherectomy - 4; multilobal - 4; corpus collosum section - 2. At 2 years follow-up 41 patients had Engel 1 status $(47 \%) ; 7$ patients engel $2(10.6 \%) ; 10$ patients Engel 3 (15.2\%); Engel 4, 16 patients (24.2\%). Quality of life measurements were highest in Engel 1 and lowest Engel 4. Complications were rare consisting of transient deficits. No deaths reported.

Conclusion: Surgery should be considered in children and adolescents with epilepsy and children with structural lesions or drug resistant seizures. 


\section{P-118}

An Outcome Study of Epilepsy Surgery at The University of Calgary Medical Centre: 94 Consecutive Cases of Temporal Lobe Resections

\section{B.S. Chan, M.A. Lee, F.E. LeBlanc (Calgary, Alberta)}

Background: Thirty percent of epileptic patients suffer intractable seizures despite appropriate regimes of drug therapy. One-third of these patients could benefit from cortical resection, with best outcome reported in temporal lobe resection. At present, surgery is offered to approximately $10 \%$ of eligible candidates.

Methods: Ninety-four temporal lobe resections were performed at the U.C.M.C. between 1981 and 1994. Outcome of the patients was categorized using Engel's classification, and was correlated with gender, age of onset of epilepsy and the use of various pre-operative studies and intra-operative techniques.

Results: Our study shows that the current success rate of temporal lobe resections performed at the U.C.M.C. is close to $90 \%$ at one year postoperation. Moreover, $90 \%$ of these patients maintain a seizure-free or rare seizure status. An improvement in the outcome of temporal lobe resection has occurred since 1986. This improvement was shown to be related to the availability of EEG telemetry and MRI confirmation of structural abnormality.

Conclusion: This study validates the surgical treatment of temporal lobe epilepsy offered at the U.C.M.C. and supports the recommendation that more candidates who qualify, be offered a surgical treatment of their intractable epilepsy.

\section{P-119}

\section{N9: Paediatric Developmental and Normative Data}

\section{L.J. MacMillan, J.E. Shaw, M.J. TAYloR (Toronto, Ontario)}

Detecting abnormalities of the peripheral nervous system, whether they be subtle or profound, is critical to accurately interpret somatosensory evoked potentials (SEPs). Recording the propagated volley passing through the brachial plexus is standard when recording clinical SEPs to median nerve stimulation and there are well established normative values for this in the adult literature. Recordings from 90 children (4 months to 18 yrs divided in 9 age groups), in response to unilateral median nerve stimulation, were obtained over the ipsilateral clavicle referenced to the opposite clavicle. The eliciting stimuli were electrical pulses of $0.2 \mathrm{msec}$ presented transcutaneously over the median nerve at the wrist at a rate of $4.1 / \mathrm{sec}$ at intensities that produced a twitch of thumb and/or finger. The bandpass used was $30-3 \mathrm{k} \mathrm{Hz}$ with 50 $\mu \mathrm{V}$ sensitivity setting. The sweep was $50 \mathrm{msec}$ and at least 2 recordings of 200 artefact-free trials were averaged. Fewer trials are almost always needed due to the larger potentials found in children and their naturally less tense state. Impedances were always below $2 \mathrm{k} \Omega$. N9 latencies, like the cervical components, change with limb growth and myelination. The latencies ranged from $5.1 \mathrm{~ms}$ in the youngest age group (4-8 mos) to $9.8 \mathrm{~ms}$ in the oldest group (16-18 yrs). A curious bifid morphology in the infant and toddler age groups was a consistent finding probably due to varying rates of maturation of different fibres. Given the variables of maturation and arm length, age and size matched normative data need to be established if one is going to test children.

\section{P-120}

The Management of Childhood Seizures by Family Physicians in Southwestern Ontario: Implications for Epidemiologic Studies

K.N. Speechley, S.D. Levin, S. Wiebe, W.T. Blume (London, Ontario)

Background: There have been no studies of the incidence of childhood seizures in Canada. It is important to identify a representative cohort with newly diagnosed epilepsies to estimate incidence and evaluate patients' quality of life. Case ascertainment through paediatricians and neurologists would be a valid method if family physicians refer all new cases of childhood epilepsy.

Methods: A random sample of family physicians practising in Southwestern Ontario participated in a mailed survey to determine if they refer children with seizures to specialists. Case histories describing seven types of childhood seizures were presented to physicians with instructions to respond whether they would: investigate/manage without referral; refer to a specialist only if problems occurred; or refer to a specialist always.

Results: $184 / 215$ family physicians (86\%) returned completed surveys. Only $1.6 \%(95 \% \mathrm{CI} 1.58-1.62)$ would not refer a child in status epilepticus, and $0.5 \%$ (95\% CI $0.47-0.53$ ) would not refer a child with worsening partial epilepsy or a child with neonatal seizures. Only 5.4\% (95\% CI 5.37-5.43) and $6.0 \%$ (95\% CI 5.97-6.03), respectively, would not refer a neurologically normal child with a first, brief generalized clonic seizure or with absence epilepsy. In contrast, only $13.6 \%(95 \% \mathrm{CI}$ 13.55-13.65) would always refer a child with a febrile seizure, while $40.2 \%$ (95\% Cl 40.13-40.27) would not refer this patient and $46.2 \%$ (95\% CI 46.13-46.27) would refer only if problems occurred.

Conclusions: Surveying paediatricians and neurologists is a valid method for recruiting a representative population-based cohort of recently diagnosed patients for epidemiologic studies of childhood epilepsy.

\section{P-121}

Children's Perception of Their Epilepsy: First Stage in the Development of a Disease-Specific Quality of Life Instrument.

G.M. Ronen, P. Rosenbaum, M. Law, D. Streiner, (Hamilton, Ontario)

Background: We lack knowledge about how children perceive their epilepsy. This report describes a research process with children with epilepsy which identified their burdens and concerns as background for developing a measure of quality of life.

Methods: 21 children with active epilepsy and no other major morbidity ages 6-10 in 5 groups, and parents in 9 groups, partic- 
ipated. The focus groups were each led by 2 moderators who facilitated the dialogue along preset questions and activities. Discussions were audiotaped and transcribed. A procedure of Text Coding was used for analysis and then validated with the original participants.

Results: We identified epilepsy-related quality of life items and domains for the new measure. Not all parents detected their children's concerns or appreciated the magnitude of their burdens. The analysis pinpointed emerging themes which deepen our understanding of the participants' perceptions.

Conclusions: School-aged children are as comfortable with focus groups as adults. Their perceptions are distinct, illustrating potential limitations with proxy accounts. The findings demonstrate the essential initial procedures in designing disease-specific quality of life instruments in childhood disorders.

\section{P.122}

\section{Cryptogenic Temporal Lobe Epilepsy: Early Course and Intractability}

\section{R. Ahmad, M. Sundaram (Jackson, Mississippi)}

Background: Information on the early course in intractable temporal lobe epilepsy (TLE) is not available and was examined in this study.

Methods: Consecutive patients with partial seizures with temporal lobe epileptiform discharges and normal neurological examination seen in our clinics between Oct.94 and Dec.95 were studied. Patients with progressive neurological disorder or significant head injury were excluded. Seizure details were obtained from the patient as well as at least one parent.

Results: Cohort included 39 patients (males - 9 , females 30 ) with mean age of 34 years(range: 19 to 60 years). Seizure onset ranged from infancy to 59 years (mean: 17 years). Follow up from seizure onset was 19 years (range :1 to 60 years). Seizures were intractable (at least 6 seizures per year for the last 4 years in spite of 3 or more anticonvulsants in adequate doses) in 35 patients and well controlled in 4.

Seizure frequency in the first 4 years of the disease was already high(at least 6 attacks per year)in 33 of 35 currently intractable patients. Febrile seizures ( 9 of 39 ) and family history of seizures among first degree relatives ( 13 of 39 ) were the only risk factors identified.

Conclusion: Seizure frequency in the early years appears already high in most patients with chronic intractable cryptogenic TLE. This has implications for treatment strategies.

\section{P-123}

\section{Accidental Injury is a Serious Risk in Typical Absence Epilepsy}

E.C. Wirrell, P.R. Camfield, C.S. Camfield, J.M. Dooley, K.E. GoRdon, (Halifax Nova Scotia)

Background: To determine if young adults with a history of typical absence epilepsy (AE) in childhood have a greater risk of accidental injury than controls with juvenile rheumatoid arthritis (JRA), and to assess the nature and severity of these injuries.

Methods: All patients with AE or JRA diagnosed between 1977-85, who were 18 years or older at study onset were identified from review of paediatric EEG records for the province of Nova Scotia (AE) or review of the medical records data base at the only tertiary care paediatric centre for the province (JRA). $59 / 69(86 \%)$ patients with AE and $61 / 76(80 \%)$ with JRA participated in an interview in 1994-95, assessing nature, severity and treatment of prior accidental injuries. Patients with $\mathrm{AE}$ were further questioned about injuries sustained during an absence seizure.

Results: $16 / 59$ (27\%) patients with AE reported accidental injury during an absence seizure, with the risk of injury being 9\% per person-year of absence epilepsy. Most injuries (81\%) occurred during anti-epileptic drug (AED) therapy. Although the majority of injuries did not require treatment, 2/16 (13\%) required minor treatment and $2 / 16(13 \%)$ were admitted to hospital. The risk of accidental injury resulting from an absence seizure in person-years at risk was highest in juvenile myoclonic epilepsy (45\%), moderate in juvenile absence epilepsy (14\%) and lowest in childhood absence epilepsy (3\%).

Patients with $\mathrm{AE}$ had a greater number of overall accidental injuries than those with JRA ( $p<0.04)$, but these differences were particularly marked for bicycle $(p<0.003)$ and car accidents $(\mathrm{p}<0.05)$ and for mild head injuries $(\mathrm{p}=0.05)$.

Conclusions: Accidental injury is common in $\mathrm{AE}$ and usually occurs after AED treatment is started. Injury prevention counselling is indicated both at diagnosis and follow-up. Bicycle accidents pose a special risk and helmet use should be mandatory.

\section{P-124}

\section{Coexistence of Hemimegalencephaly and Chronic Encephalitis}

V. Jay, H. Otsubo, P. Hwang, H. Hoffman, S. Blaser, M. ZiELENSKA (Toronto, Ontario)

We report the extraordinary association of hemimegalencephaly with chronic encephalitis and cytomegalovirus (CMV) positivity in a 5-month-old infant with intractable seizures and a left hemispheric resection. Microscopy revealed a severe neuronal migration disorder (NMD) with fusion of gyri, marked disarray of neuronal lamination, neuronal gigantism and extensive neuronal heterotopias. Also widespread were microgial nodules, gliosis and nodular calcifications and some foci of frank necrosis with calcification. Occasional perivascular and leptomeningeal lymphocytic infiltrates were present. No viral inclusions were identifiable. Polymerase chain reaction on multiple specimens showed unequivocal CMV positivity. In intrauterine CMV infection, NMDs such as polymicrogyria are well recognized, but the association of hemimegalencephaly with CMV infection has not been previously described. Our finding of chronic encephalitis with CMV positivity and hemimegalencephaly in the same patient, raises questions about the role of CMV in the etiopathogenesis of the NMD. 


\section{P-125 Withdrawn}

\section{P-126}

\section{Epilepsia Partialis Continua as the First Manifestation of Multiple Sclerosis}

D. Rivest, M. Thibault, D. Brunet, R. Desbiens, J.P. BOUCHARD (Quebec, Quebec)

Background: Although epilepsy is more common in persons with multiple sclerosis (MS) than in the general population, it is seldom the initial symptom of MS. In MS-associated epilepsy, seizures are of limited duration and respond well to antiepileptic drugs. We report a patient who presented epilepsia partialis continua (EPC) as the first manifestation of MS.

Case report: A 30 year old man was admitted with continuous clonic movements of the left foot. His medical history was unremarkable. The neurological examination was normal. Cerebral MRI revealed white matter lesions compatible with MS with a cortico-subcortical plaque in the parasagittal right frontal lobe. EEG-EMG recordings showed focal periodic epileptiform discharges in the parasagittal right frontal region preceding the myoclonic jerks (latency : $100 \mathrm{~ms}$ ). CSF studies revealed two oligoclonal bands. EPC was refractory to phenytoin and carbamazepine. We observed a slight improvement with clonazepam and I.V. prednisolone therapy at discharge.

Conclusion: EPC has been associated only exceptionally with MS. We postulate that an acute demyelinating lesion at this particular region created a deafferentation of the cortex and subsequently EPC. At one month follow up, the seizures were greatly reduced suggesting that the prognosis may be better in EPC when associated with MS than with other causes.

\section{P-127}

\section{The Prognosis of Cryptogenic West Syndrome with Normal MRI}

\section{B.L. BANwElL (Toronto, Ontario)}

Background: Cryptogenic West Syndrome (WS) is characterized by lack of previous brain damage, no known etiology, and by a normal CT scan. Normal MRI results have not been routinely required for cryptogenic diagnosis, and little is known of the prognostic utility of MRI in this population.

Methods: A detailed chart review of 132 patients diagnosed with WS was performed. Cryptogenic WS occurred in 34 children. CT and MRI studies were reviewed in a blinded fashion.

Results: Cognitive development was reported to be normal in 16 children, significant language delay in 7 , and 7 children were non-verbal. 21 children were seizure-free, 6 children have intractable seizures, 2 have myoclonic jerks, and 5 had insufficient follow-up. MRI scans were performed in 17 patients. Normal MRI results were found in 10 cases, 4 of whom are developmentally normal, 5 are profoundly delayed, and 1 lacks follow-up data. Of the 7 abnormal MRI scans, 2 showed heterotopic grey matter, the remaining 5 scans showed mesial tempo- ral asymmetry or mild volume loss. Cognitive outcome data is available for 4 of these patients, 3 are delayed.

Conclusions: We conclude that a normal MRI does not aid in the prognosis of children with WS as defined by our inclusion criteria. We further suggest that the cognitive outcome, even when early milestones appear normal, may show significant deficits. Detailed neuropsychological testing is currently underway.

\section{P-128}

\section{Meningioangiomatosis: An Electrophysiological Perspective}

S. Wiebe, D. Munoz, J.P. Girvin, W.T. Blume, R.S. MCLACHLAN (London, Ontario)

Background: Meningioangiomatosis (MA) is a benign lesion affecting cerebral cortex and leptomeninges. It often causes early onset intractable seizures. Our objective was to identify its clinical, electrographic and laboratory features, and surgical outcome.

Methods: Case identification of pathology records. Analysis of clinical, ictal, EEG, and imaging features. Retrolective outcome assessment and telephone contact.

Results: Six patients were identified. Age at seizure onset and surgery ranged from 8-26 and 10-37 years respectively. Follow-up ranged from 2-17 years. Location of MA was: 3 temporal, 1 insular, 1 inferior-posterior Rolandic, 1 mesial parietal occipital. Mesial temporal sclerosis (MTS) occurred in patients with temporal lobe lesions. Von Hippel Lindau existed in one patient's family. One patient presented with lesional haemorrhage. Surgical outcome was influenced by extent of resection and seizure duration. Four/six patients are seizure free, three off medications. Two/six obtained a significant improvement $(>75 \%)$. Pathological diagnoses in early cases included glioblastoma multiforme and hamartoma. Electrographic and pathological features will be described. MTS and epileptogenesis often accompany extramesial temporal MA.

Conclusions: Complete resection appears more important for seizure control than previously appreciated. Neurofibromatosis is infrequent in this cohort presenting with seizures. MA may be associated with other phakomatoses.

\section{P.129}

\section{Reversible Downbeat Nystagmus due to Lamotrigine} Toxicity

E. Tasch, A.L. Lafontaine, A. Bernasconi, T. Kirkham, A. Sherwin, F. ANDERMANn (Montreal, Quebec)

The most commonly reported adverse experiences with lamotrigine are dizziness, headache, nausea, and rash. Rarely patients complain of blurred vision, diplopia, and ataxia. Downbeat nystagmus has not been previously reported. The therapeutic/toxic range of lamotrigine is unestablished.

A 20 year-old woman with idiopathic generalized epilepsy, taking valproic acid and lamotrigine, developed nausea, vomiting, and truncal ataxia. She also had downbeat nystagmus in primary position with exacerbation on oblique and downward gaze, and no nystagmus on upgaze. Daily serum concentrations of 
lamotrigine and valproic acid were measured using high performance liquid chromatography. With a daily dose of $300 \mathrm{mg}$ of lamotrigine and $1500 \mathrm{mg}$ of valproic acid the serum levels were 17.1 micrograms per milliliter and 384 micromoles per liter respectively. Reduction of the lamotrigine dose resulted in resolution of the downbeat nystagmus with levels under 10 micrograms per milliliter. MRI of the brain was normal.

Levels of lamotrigine over 10 micrograms per milliliter were associated with downbeat nystagmus and ataxia which resolved on reducing the dose. Downbeat nystagmus may occur with several different antiepileptic drugs, and lamotrigine can now be added to the list.

\section{P-130}

\section{Landau Kleffner Syndrome: Treatment with Ketogenic Diet}

\section{C.L. Voll, J. YaGer, N. LowRY (Saskatoon, Saskatchewan)}

We describe an 8 year old female whose neurological and language development before onset of illness is well documented, and who, at age 22 months, developed acquired aphasia with epilepsy and a paroxysmal EEG (Landau-Kleffner syndrome).

At 4 years she was totally unable to communicate through speech. Her EEG showed frequent bilateral independent temporal spike and wave discharges becoming almost continuous during sleep. She continued to have frequent seizures with recurring episodes of status epilepticus and became increasingly impaired in communicative skills, despite treatment with corticosteroids and many anticonvulsant drug combinations.

Because of worsening seizures, at 7 years, all drugs were discontinued. She was placed on a ketogenic diet, according to the John Hopkins protocol. Since that time, she has remained essentially seizure free (one minor partial seizure after a lapse in diet 6 months after starting the diet). She has shown as well a marked improvement in behavioral, motor, and particularly language function.

Although follow up at this time is limited to only twelve months, we are very encouraged by her continuing improvement off all medication. Certainly this suggests that further exploration of the use of this seldom-used treatment for LandauKleffner syndrome is warranted.

\section{P-131}

\section{Intractable Cryptogenic Hemispheric Epilepsy}

\section{W.T. BLUME (London, Ontario)}

Background: Although the principal categories of the International Classification of Epileptic Seizures and Epilepsies are partial and generalised, some patients occupy a mid position between these categories.

Results: Four patients (ages 23-42 years) have had intractable seizures without associated central nervous system abnormalities beginning at ages 5-15 years. Complex partial, ipsi- and contra-versive seizures occurred in all. EEGs in each patient showed hemispheric spike and waves, bisynchronous spike- waves, and multifocal spikes, principally in the hemisphere of spikewaves. Left frontal subdural recording in one showed widespread spike-waves. MRIs were normal. All patients remained uncontrolled on two antiepileptic drugs.

Conclusion: Regionally accentuated seizures without demonstrable lesions may be intractable.

\section{P.132}

\section{Persistent Epilepsy from Head Trauma Without Coma}

M. SADLER (Halifax, Nova Scotia), W.T. Blume (London, Ontario), F. ANDERMANN (Montreal, Quebec)

Background: High risk factors for the development of late post-traumatic epilepsy include penetrating missile brain injury, early seizures, depressed skull fracture, Glasgow Coma Scale $\leq$ 10, intracerebral hematoma, and epi/subdural hematoma. We describe 4 patients with persistent seizures developing within months of head trauma without the usual risk factors for late post-traumatic epilepsy.

Results: Male $=1 ;$ female $=3$. Age at time of trauma $=9-25$ years. Followup since trauma $=9-36$ years. Patient $I$ fell backwards hitting his occiput; patient 2 hit her head on a steering wheel; patient 3 was hit by a baseball bat; patient 4 fell off a horse. None were unconscious; $1 / 4$ sought immediate medical treatment (dental work only); $1 / 4$ had posttraumatic amnesia (< 2 hours). No patient had "early" seizures. All patients developed partial complex seizures and secondarily generalized seizures 26 months after trauma. $3 / 4$ had medically resistant seizures. 1/4 has a severe anterograde memory disturbance. All patients had EEG abnormalities (interictal spikes, focal slowing, recorded seizures) involving > one temporal lobe. All had CT scans after epilepsy began; $1 / 4$ had an abnormality (temporal cyst). 1/3 with MRI had an abnormality (bilateral increased hippocampal signal and atrophy). $2 / 3$ were rendered seizure free with surgery (still on medication; followup 6 and 12 years).

Conclusion: Persistent temporal lobe epilepsy may be a result of head trauma lacking the usual indices of unfavorable prognosis.

\section{P.133}

\section{Seizures In CNS Tuberculosis}

Z. Arvanitakis, E. Hershfield, N. Pillay, C. Power (Winnipeg, Manitoba)

Objective: To define the clinical profile, risk factors and outcome of patients with CNS tuberculosis (TB) complicated by seizures.

Background: Seizures are frequently associated with cerebral tuberculomas, but not with TB meningitis. However, the clinical features of seizures associated with CNS tuberculosis have not been defined.

Methods: A case-control retrospective analysis of adult patients with CNS tuberculosis, stratified by the presence or absence of seizures, was performed. Patients were ascertained from the Tuberculosis Control Services, Manitoba Lung Association from 1982 to 1995 and were matched for age, 
ethnicity, and CNS-TB (tuberculoma or meningitis) based on clinical presentation, CSF findings, and neuroimaging.

Results: We identified 24 patients with CNS-TB: TB meningitis [TBM, $\mathrm{N}=15$ ] and brain tuberculoma [BT, $\mathrm{N}=9$ ]. $50 \%$ of patients were Aboriginal and $25 \%$ were recent immigrants. Twelve of the twenty-four patients had seizures (TBM, N=6). Focal seizures were observed in $50 \%$ of patients. The development of seizures was associated wiht a lower CSF cell count $(\mathrm{p}<0.005)$. All patients with seizures had an abnormal EEG with focal abnormalities in $65 \%$ of patients and epileptiform activity in $50 \%$ of patients. All patients with seizures were treated with phenytoin without recurrence of seizures. Mortality and morbidity was higher in the seizure group.

Conclusions: The risk of seizures was higher in TB meningitis than previously reported. The occurrence of seizures was closely associated with EEG abnormalities. Low CSF cell counts were predictive of seizures complicating CNS tuberculosis.

\section{P.134}

Infantile Spasms Secondary to a Thalamic Arteriovenous Malformation

T. Balslev, M. Cortez, H. Kolski, H. Otsubo, S. Blaser (Toronto, Ontario)

Background: Infantile Spasms (IS) are a clinical response of the immature brain to CNS insults. IS secondary to a thalamic arteriovenous malformation (AVM) has not been previously reported.

Patient: This term female infant was delivered by a cesarean section due to breech presentation. No signs of fetal distress were noticed. She presented with an early right-handed preference, rigidity of the left arm, a mild developmental delay and clusters of extensor spasms at the age of 7 months.

Results: EEG on admission showed hemihypsarrhythmia. CT and MRI disclosed a volume loss and an AVM in the right thalamus. MRA and conventional angiogram demonstrated abnormal vessels originating from the lateral posterior choroidal arteries. Good seizure-control was achieved on monotherapy with nitrazepam. At follow up, a moderate developmental delay was found.

Conclusion: Infantile spasms can be produced by a unilateral thalamic AVM in the absence of a structural cortical abnormality. We hypothesize that a thalamic lesion may influence the ipsilateral cerebral cortex through the thalamo-cortical radiations, and discuss the findings in the light of the current hypotheses on the pathogenesis for IS.

\section{P-135}

Psychosis in the Presence of Repetitive Electrographic Left Temporal Lobe Seizures During Stage II Sleep in a Fourteen Year Old Girl

D.W. Gross, N.J. Lowry, C. Wood (Saskatoon, Sask.)
Background: Psychosis has been described in association with epilepsy, however, controversy still exists regarding whether psychosis is more common in temporal lobe epilepsy as opposed to other types of epilepsy, and whether psychosis, in epileptics, is more commonly associated with increased or decreased seizure frequency.

Methods: We present the case of a 14 year old girl who presented with a second episode of prolonged psychosis, and was subsequently found to have electrographic seizures.

Results: Electroencephalography (EEG), while the patient was awake, showed theta slowing with occasional spikes and sharp waves, as well as two short electrographic seizures in the left anterior temporal lobe with no clinical accompaniment. EEG telemetry demonstrated almost continuous electrographic seizure activity originating from the left temporal lobe during stage II sleep. MRI scan was normal. The patient's symptoms improved gradually over several weeks, while being treated with phenytoin and valproic acid, despite little improvement in her overnight EEG telemetry.

Conclusions: This case demonstrates episodic psychosis in the presence of left temporal lobe seizures. With the high frequency of electrographic seizure activity at the time of the psychosis, it would be presumed that, in this patient, psychosis was related to increased frequency of electrographic seizures during stage II sleep.

\section{P-136}

Ehlers Danlos Syndrome in a Patient with Congenital Bilateral Perisylvian Syndrome: Coincidence or Causation

\section{A.L. Lafontaine, E. Tasch, F. Andermann (Montreal, Quebec)}

Congenital bilateral perisylvian syndrome (CBPS) occurs in patients with congenital facio-pharyngo masticatory diplegia, epilepsy, mental retardation and congenital limb deformities. Ehlers-Danlos syndrome (EDS) is a heritable disorder of the connective tissue characterized by hyperextensible skin, hypermobile joints, fragility, and bruisability.

A 20 year-old woman with intractable epilepsy and an unusual phenotype consisting of an expressionless face, open mouth, large lips, marked reduction in tongue movements, and anarthria was discovered to have bilateral perisylvian polymicrogyria on MRI. She also had joint laxity and skin hyperextensibility clinically typical of EDS type II.

It had been suggested that polymicrogyria is secondary to a disorder of neuronal migration. The establishment of an intact basement membrane is essential to neuronal migration. The basement membrane is formed by the interaction of laminins, fibronectins, and type IV collagen. Although the molecular defect in EDS type II is not known, mutations in collagen and fibronectin have been proposed. We suspect that the association of these rare congenital disorders are caused by a common genetic mutation. 
P-137

Effects of Summer Camp on the Quality of Life in Children with Epilepsy. (CANN)

A. Allen, T. Mertineit

\section{EXPERIMENTAL THERAPEUTICS}

\section{P-138}

Expression of Myc and p53 Genes Following Transient Global Ischemia

L. McGahan, G.S. Robertson, A.M. Hakim (Ottawa, Ontario)

Background: The proto-oncogene c-myc and the tumor suppressor gene $\mathrm{p} 53$ encode transcriptional regulating factors implicated in apoptosis. The present study examined whether the protein products of these genes are expressed in neurons that undergo delayed death following global ischemia.

Methods: Four vessel occlusion was produced in male Wistar rats by cautery of the vertebral arteries and clamping of the common carotids for $20 \mathrm{~min}$. Animals were sacrificed 2, 12, 24, 48 and $72 \mathrm{~h}$ following recirculation.

Results: Immunohistochemical staining revealed elevated p53-like immunoreactivity (-LI) in the hippocampus (24-48 h); as well as vulnerable areas of the amygdala, thalamus, and cortex $(12-48 \mathrm{~h})$. At $24 \mathrm{~h}$, there was a significant increase in the number of p53-LI nuclei in both vulnerable $\mathrm{CA}_{1}(64 \pm 4.0)$ and more resistant $\mathrm{CA}_{3}(37 \pm 2.6)$ neurons of the hippocampus $(p<0.01)$. At $48 \mathrm{~h}, \mathrm{p} 53-\mathrm{LI}$ was still elevated in $\mathrm{CA}_{1}(70 \pm 7.1$, $\mathrm{p}<0.01$ ) neurons but had subsided to basal levels in $\mathrm{CA}_{3}$ neurons. In contrast, MycLI was selectively elevated in vulnerable $C A_{1}$ neurons $48-72 \mathrm{~h}$ after ischemia.

Conclusions: The close temporal correlation between these changes in gene expression and the loss of $\mathrm{CA}_{1}$ neurons suggests that increases in p53 and Myc levels may contribute to the delayed death of $\mathrm{CA}_{1}$ neurons following global ischemia. (Supported by London Life and the Heart and Stroke Foundation of Ontario.)

\section{P-139}

\section{Dehydroepiandrosterone (DHEA) Prevents Ischemic Neuronal Injury}

H. LI, P. Sun, A.M. Buchan (Calgary, Alberta), D. Small (Ottawa, Ontario)

Background: DHEA has been shown to extend life and improve the deterioration from a number of neurodegenerative conditions. In addition, it prevents injury from a NMDA induced excitotoxic lesion.

Methods: Male wistar rats $(100 \mathrm{~g})$ were pretreated with an implanted pellet of DHEA for 12 days at $25 \mathrm{mg} /$ rat (group 1), $50 \mathrm{mg} /$ rat (group 2), and $100 \mathrm{mg} /$ rat (group 3). On day 13 rats were exposed to normothemic 4 vessel occlusion ischemia for
10 mins. and were subsequently reperfused for 7 days and then histologically examined for $\mathrm{CA}_{1}$ neuronal injury.

Results: The results demonstrate a neuroprotective response for DHEA following transient forebrain ischemia. $\mathrm{CA}_{1}$ injury has attenuated.

Conclusions: DHEA may be a useful strategy following early treatment with either t-PA or an NMDA antagonist as a way of preserving neurones which with current therapy may have their cell death postponed rather than protected in the absolute sense. A 28-day study is currently underway to see if long-term protection, i.e. not just postponement of injury can be afforded. The results will be presented. (Funded by HSFO, MRC and HSFA)

\section{P-140}

An In Vitro Excitotoxin Assay System Using the Human NT. 2 Teratocarcinoma Cell Line

T.J. SChrader, C. Héroux-MetCalf and I. Langlois (Ottawa, Ontario)

Background: Following a 1987 outbreak of Amnesic Shellfish Poisoning in Canada, the need was recognized for an in vitro assay able to detect and assess excitotoxic food-associated chemicals. Retinoic acid induces NT-2 cell differentiation into nonreplicating neuronal cells which are sensitive to glutamate and kainate, thus providing a potentially useful assay system.

Methods: NT-2 cells are differentiated by incubation in the presence of $10 \mu \mathrm{M}$ retinoic acid for 5 weeks. Following differential harvesting, the cells are seeded into 96-well plates and incubated in the presence of $20 \mu \mathrm{M}$ cytosine arabinoside for 1 week followed by a medium change. After test chemical addition, excitotoxicity is assessed by microscopic evaluation of phenotypic changes as well as by increased propidium iodide (PI) fluorescence.

Results: In culture, the differentiated cells form distinct ganglia-like formations joined by smooth axon-like projections. Addition of glutamate agonists causes cell swelling by $30 \mathrm{~min}$ utes and progressive cell deterioration over 24 hours, resulting in cell remnants with axons exhibiting extensive striations and blebbing. PI fluorescence becomes detectable after 4 hours and increases with time. Concentration/fluorescence profiles have been generated for L-glu, DL-asp, AMPA, NMDA, kainate, domoate and L-cys. Most agonists become toxic at $50 \mu \mathrm{M}$, with the exceptions of domoate $(5 \mu \mathrm{M})$ and L-cys $(500 \mu \mathrm{M})$. The agonists also differed in the level of PI fluorescence observed (kainate $=$ domoate $=$ asp $>$ glu $>$ NMDA $=$ AMPA). L-glu and kainate toxicities can be further distinguished by differential inhibition using MK801 and DNQX.

Conclusions: The NT-2 cell line provides a ready supply of differentiated human neuronal cells exhibiting sensitivity to a wide spectrum of excitotoxins. Excitotoxicity is monitored both visually and by changes in PI fluorescence, and the assay should prove useful in toxicological testing programs. 
Rational Design of a Neurotrophin Antagonist: In Vitro Effects and Basis of Activity

R.J. Riopelle, G.M. Ross, I. Shamovsky, D.F. Weaver (Kingston, Ontario)

Background: Published data on structure and function of neurotrophins suggest that these dimeric proteins display distinct functional domains that interact with receptors and that confer dimer integrity.

Methods: A $2.3 \mathrm{~A}^{0}$ crystal solution of the prototypic neurotrophin NGF has been used to design a conformationally constrained hybrid peptide homologue of putative functional domains of this neurotrophin.

Results: The synthetic bicyclic peptide inhibited NGF-mediated neurite growth in vitro with no influence on neuronal survival in a 24-hour assay. The peptide also inhibited chemical cross-linking of NGF to its receptors trkA and p75, and blocked NGF-stimulated trkA phosphorylation. The mechanism of action of the peptide appears to involve disruption of the dimer integrity of NGF

Conclusions: The present observations are the first to demonstrate the necessity for dimer integrity for NGF function, and provide a strategy for design of pure antagonists of neurotrophins. A number of potential therapeutic strategies emerge from these observations (see companion abstract, Riopelle, Ross, Weaver, Bisby, Fahnestock, Racine).

Supported by the Neuroscience Network, MRC (Canada), and Allelix Biopharmaceuticals (Mississauga, Ontario).

\section{P.142}

\section{A Peptide Homologue of a Neurotrophin Receptor Domain Potentiates NGF Effects In Vitro}

\section{R.J. Riopelle, S.M. Dostaler, G.M. Ross, D.F. Weaver} (Kingston, Ontario)

Background: The common neurotrophin receptor $\mathrm{p} 75$ and some other receptors of the NGF/TNF receptor superfamily contain amphiphilic motifs in cytoplasmic domains (Myers, S.M., et al., 1994. Putative cytoplasmic amphiphilic domains in the nerve growth factor receptor superfamily Biochem. Biophys. Acta 1196:21-28).

Methods: To analyze the function of the putative amphiphilic motif of p75 in NGF signalling, we synthesized a peptide homologue, and demonstrated that it adopted predicted structure, and partitioned into neuronal cells.

Results: In the presence of NGF, the peptide, designated R-3, enhanced neurite growth, and shifted the NGF dose response for neurite growth to the left. R-3 had no effect on NGF binding, uptake, or phosphorylation of the NGF specific receptor trkA. The effects of $\mathrm{R}-3$ were dependent upon neuronal expression of trkA, and relationships between charged residues within the peptide structure.

Conclusions: Potentiation of the effects of limiting concentrations of neurotrophin with small compounds that partition through the lipid bilayer may have therapeutic implications.

Supported by the Neuroscience Network.
Small Molecule Mimetics of Glycosaminoglycan Binding Domains as Antagonists of Dystrophic Neurite Growth

R.J. Riopelle, S.M. Dostaler, A. Borrajo, G.R.J. Thatcher, (Kingston, Ontario)

Background: Proteoglycans (PG's) are complex carbohydrate containing glycoproteins present on the neuronal cell surface and in the extraneuronal milieu in the central nervous system that have been implicated in neurite growth. Since dystrophic neurite (DN) formation is a prominent correlate of clinical severity in AD, and since PG's are involved in neurite growth, and are found as constituents of DN's, we have targetted these complex glycoproteins to develop antagonists that might offer an opportunity for future therapeutic intervention in AD.

Methods: To mimic binding sites for glycosaminoglycan (GAG) domains of neuronal PG's, a series of soluble polyammonium (SPA) species were covalently coupled to a naturally occurring platform in a known regiochemistry.

Results: In solution these compounds inhibited NGF-mediated neurite growth on poly-D-lysine (PDL), but had no effect on cell adhesion to this substrate. When immobilized on tissue culture plastic, these compounds promoted adhesion and neurite growth approximately inversely proportional to their abilities to inhibit neurite growth when in solution. The biological properties of the compounds could not be correlated solely with charge density provided by the SPA's, suggesting that innate properties of, and conformational constraints imposed by the platform, and the regiochemistry employed, were functionally significant. That the compounds inhibited neurite growth on, but not adhesion to, PDL suggested specificity for HSPG's; such specificity was confirmed for one of the compounds.

Conclusions: These observations with soluble mimics of GAG-interactive motifs provide insights into the molecular basis of interactions supporting neuronal adhesion and neurite growth, and the structural requirements for specificity of function. (Supported by MRC Canada.)

\section{GENERAL NEUROLOGY}

\section{P-144}

\section{Tremor in Klinefelter's Syndrome1}

\section{A. KIRK (Saskatoon, Saskatchewan)}

Background: Several previous observations have suggested an association between Klinefelter's syndrome and essential tremor. Some studies have suggested that the association is coincidental.

Methods: Two young men with Klinefelter's syndrome and essential tremor are herein described.

Results: A 21-year-old man presented with gradually worsening tremor over 7 years. A 27 -year-old man presented with gradually worsening tremor over 4 years. In both patients the tremor resembled essential tremor and affected the hands symmetrically. Both patients had a good response to Propranolol. 
Neither patient had a family history of tremor or other neurologic disorders.

Conclusions: Patients with Klinefelter's syndrome may present with essential tremor.

\section{P-145}

Inhibition of Calmodulin Dependent Cyclic Nucleotide Phosphodiesterase Isoymes by Deprenyl

R. Kakkar, R.V.S. Raju, A.H. Rajput and R.K. Sharma (Saskatoon, Saskatchewan)

Background: Calmodulin-dependent cyclic nucleotide phosphodiesterase (CaMPDE) is one of the key enzymes involved in the complex interactions between cyclic nucleotide and $\mathrm{Ca}_{2}{ }^{+}-$ second messenger systems. CaMPDE exists in different isozymic forms which exhibit distinct molecular and/or catalytic properties. They are designated according to the tissue origin and the subunit molecular mass as brain $63 \mathrm{kDa}$, brain $60 \mathrm{kDa}$, heart and lung CaMPDE isozymes.

Results: The effects of Deprenyl (selegiline hydrochloride) which is believed to be selective inhibitor of monoamine oxidase B, on CaMPDE isozymes was investigated on bovine tissue. Our findings indicate that Deprenyl inhibited brain $60 \mathrm{kDa}$, heart and lung CaMPDE isozymes. However, the inhibition for brain $63 \mathrm{kDa}$ was observed at only higher concentrations of Deprenyl. The inhibition of brain $60 \mathrm{kDa}$ CaMPDE was overcome by increasing the concentration of calmodulin suggesting that Deprenyl is calmodulin antagonist.

Conclusion: Therefore, Deprenyl would be a valuable tool in investigating pathogenesis of Parkinson's disease.

\section{P-146}

Effects of Inhibition of Catechol-O Methyltransferase (COMT) on the Rates of Uptake $\left(K_{i}\right)$ and Reversibility $\left(k_{\text {loss }}\right)$ of 6- fluoro -L-dopa (FDOPA) in the MPTP-primate Model of Parkinson's Disease

D.J. Doudet, G.Y.L. Chan, J.E. Holden, T.J. Ruth (Vancouver, British Columbia), R.J. WYaTT (Bethesda, Maryland)

Background and Methods: The extended graphical analysis combining brain and plasma FDOPA PET data allows the measurement of the striatal uptake rate constant $\mathrm{K}^{\mathrm{i}}$ and the catabolic rate constant $\mathrm{k}^{\text {loss }}$ of FD. We examined $\mathrm{K}^{\mathrm{i}}$ and $\mathrm{k}^{\text {loss }}$ in 6 normal and 6 unilaterally MPTP-treated rhesus monkeys with and without premedication with the peripheral and central COMT inhibitor Ro 40-7592 (8mg/kg i.p. 2hrs before FDOPA administration) to explore its possible central effects in primates and its potential as adjunct to levodopa therapy. Each animal received two 3hr-Jong FDOPA studies, one in each condition.

Results: In both conditions, $\mathrm{K}_{\mathrm{i}}$ was significantly decreased and $k_{\text {loss }}$ was significantly increased (p. <0.05) in the MPTPtreated striatum compared to the unlesioned striatum and controls. There was no significant difference between the uptake rate constants in normal and MPTP-treated animals with and without premedication with the COMT inhibitor. However, there was significant decrease $(40 \% ; p<0.003)$ in the catabolic rate constant in the MPTP-lesioned striatum with Ro40-7592 pretreatment $(0.0037 \pm 0.0015 \mathrm{~min}-1)$ compared to the MPTPlesioned striatum alone $(0.0049 \pm 0.0015 \mathrm{~min}-1)$.

Conclusions: The increased rate of reversibility $k_{\text {loss }}$ in the parkinsonian animals is in keeping with the hypothesis of compensatory increase in DA turnover in parkinsonism. The increased F-18 trapping in the striatum observed with pretreatment with Ro 40-7592, may reflect the inhibition of striatal fluoro-dopamine metabolism by COMT, thus suggesting that central COMT inhibition may provide clinical benefit by preserving striatal dopamine. These data also suggest that the extended graphical method is a viable approach to the estimation of the rate of reversibility of FDOPA trapping in the striatrum over a 3 hour period and that $\mathrm{k}_{\text {loss }}$ as measured with FDOPA PET may be useful to explore presynaptic compensatory mechanisms.

\section{P-147}

Striatal D2 Receptor Upregulation in Levodopa-unresponsive Parkinsonism Striatal D2 Receptor Upregulation in Levodopa-unresponsive Parkinsonism

R. De la Fuente-Fernandez, A. Kishore, D.B. Calne, B. LEGG (Vancouver, British Columbia), Z.K. WSzolek (Omaha, Nebraska)

Objective: To study the pre and post-synaptic dopaminergic function in a familial form of levodopa-unresponsive parkinsonism.

Background: Pallido-ponto-nigral degeneration (PPND) is an autosomal dominant form of levodopa-unresponsive parkinsonism. Pre-synaptic striatal dopaminergic function has been found to be reduced by FD PET. The regional distribution of presynaptic dopaminergic dysfunction within the striatum and the status of dopaminergic receptors in PPND have not been studied.

Methods: Two levodopa-naive members from the family with PPND (ages $43 \& 45$ years, duration of disease 2 years in both) were studied with $\left[{ }^{18} \mathrm{~F}\right] \mathrm{FD}$ and $\left[{ }^{11} \mathrm{C}\right]$ Raclopride PET scans. FD scans were analyzed using a graphical model with plasma input function to calculate the striatal uptake constant (Ki). Raclopride scans were analyzed by a target/background ratio.

Results: Both subjects showed severe reduction of fluorodopa uptake in the striatum (caudate $48 \%$ and putamen $42 \%$ of normal, both less than $3 \mathrm{SD}$ of normal mean), and an increase in raclopride binding (caudate $130 \%$ and putamen $138 \%$ of normal, both more than 2 SD of normal mean).

Conclusion: (1) Pre-synaptic nigrostriatal dysfunction in PPND shows a distribution similar to other levodopa-unresponsive parkinsonian syndromes such as PSP and SND with involvement of both caudate and putamen. (2) The post-synaptic D2 receptor system is upregulated in both caudate and putamen in PPND. This is in marked contrast to the reduced raclopride binding seen in other levodopa unresponsive -parkinsonian syndromes. The levodopa unresponsiveness in PPND is due to factors distal to the D2 receptors and is probably related to the pathology in the globus pallidus. 


\section{P-148}

\section{Saccadic Error in Balint's Syndrome: A Quantitative Study}

\section{N.Torun, J.A. Sharpe, J.J.S. Barton (Toronto, Ontario)}

Background: Balint's syndrome, which is characterized by optic ataxia, visual inattention, and "psychic paralysis of gaze" signifies bilateral parietooccipital lesions.

Methods: A 52-year-old patient had Balint's syndrome, following ischemic lesions shown on MRI. Vision was 20/30 in both eyes. She had simultanagnosia. She misreached visual targets but did not have neglect. We recorded her eye movements using a magnetic search coil technique. Horizontal saccades to targets that were random in amplitude and duration were recorded. Predictable targets were presented in three paradigms: 1) the gap task, 2) overlap task and 3) target steps without gap or overlap. Self-paced saccades between two targets 20 degrees apart were also recorded.

Results: The mean latency of saccades to targets unpredictable in amplitude and time was prolonged, with poor accuracy. Prediction did not improve latency or accuracy. The latency of the saccades to predictable targets with the central fixation light on (overlap task) was also prolonged and did not return to normal with the step or gap task.

Conclusion: The delay of visually-guided saccades in Balint's syndrome was distinctive and had no element of genuine spasm of fixation. Since reflexive visually-guided saccades were also impaired, the patient had no ocular motor apraxia either.

\section{P-149}

\section{Recovery Patterns and Lesion Localization in Sensory Extinction}

\section{P.L. EberT, S.E. Black, L.LeE (North York, Ontario)}

Background: In sensory extinction (SE), the contralesional stimulus is ignored when right and left sensory fields are simultaneously stimulated. Little is known about SE recovery patterns.

Methods: 271 consecutive stroke patients were assessable for tactile, visual, and auditory SE and hemispatial neglect within 14 days of onset. Patients with SE were recalled at 1,3 , and 12 months.

Results: SE was present in $46 \%$ of right hemisphere damage (RHD) and $26 \%$ of left hemisphere damage (LHD) subjects. In RHD with SE, $43 \%$ demonstrated single (c.f. $72 \%$ in LHD), $42 \%$ double (c.f. $25 \%$ in LHD), and $15 \%$ triple modality extinction (c.f. $3 \%$ in LHD). $80 \%$ of RHD and $61 \%$ of LHD subjects with SE also demonstrated hemispatial neglect. Approximately $2 / 3$ of the subjects with SE recovered by 3 months with little change at 1 year. Overall, 13\% (16/122) RHD subjects had persistent SE at 1 year. CT lesion overlapping indicated that white matter at the temporo-occipitopartietal junction was critical site of damage for SE.

Conclusion: SE is more common and frequently multimodal with RHD. It can be a primary or attentional deficit which recovers in the majority by 3 months. Its impact on functional status is relevant for rehabiliation planning and is currently being analyzed.

\section{P-150}

\section{Lesion Localization in Apraxia}

S.E. Black, K.L. Barbour (North York, Ontario), E.A. RoY (Waterloo, Ontario)

Background: Apraxia, a disorder of skilled movement, is thought to arise from left frontal and/or parietal damage, but few studies have examined this in a large consecutive stroke sample using standardized tasks with explicit scoring criteria.

Methods: We studied gestural pantomime in 116 consecutive stroke patients with a videotaped, standardized battery. Admission CT was parallel to the orbitomeatal line allowing sterotactic localization by the Talairach method.

Results: In the 63 patients ( 37 right, 26 left) with CT lesions analyzed to date, 14 of left hemisphere-damaged patients had severe apraxia. Lesion were overlapped in the $11 / 14$ patients with postive CT scans on templates from Talairach \& Tournoux stereotactic atlas. The most common overlap site $(6 / 11)$ was in the corona radiata deep to the temporal and parietal cortex involving the long association tracts (superior longitudinal and frontal occipital fasiculi). In $3 / 11$ overlap also occurred in the superior temporal, inferior frontal and parietal cortex and underlying deep white matter (Brodman areas 22, 40, 44). Nonapraxic and apraxic right hemisphere-damaged patients showed no overlap in the above areas.

Conclusions: If confirmed in our full sample, these findings suggest that integrity of the long association tracts, rather than cortical regions as traditionally thought, may be critical for normal praxis.

\section{P-151}

\section{Sleep Disturbance Comorbidty in Atypical Facial Pain (AFP)}

D. Stirling, H. Tenenbaum, M. Shandling (Toronto, Ontario)

Background: Patients with AFP have clinical similarities to patients with Fibromyalgia(FM). While sleep disturbance is an important feature of chronic, musculoligamentous pain and FM, this is not clearly documented in AFP. We hypothesized that the prevalence of sleep disturbance is greater in AFP patients than in the general population $(10 \%)$ and will be similar to that observed in patients with FM(60\%).

Methods: We performed a retrospective chart review determining the prevalence of subjective sleep disturbance symptoms in patients with AFP, as compared to patients seeking treatment of chronic migraine, another chronic pain condition.

Results: Using IHS criteria, a consecutive chart review was performed, identifying 20 patients with AFP and 20 patients with chronic migraine. The presence or absence of sleep disturbance complaints was extracted from the chart of each patient. Results reveal that while only $7 / 20$ patients with migraine 
reported difficulty with sleep, 15/20 patients with AFP reported sleep difficulties, a 2 fold increase in the prevalence of subjective sleep disturbance ( $p<0.02$, Fisher's Exact Test).

Conclusions: The prevalence of AFP-associated sleep difficulties reported, parallels that observed in FM and myofacial pain (Moldofsky H, 1975; Harness DM 1990). Proposed studies are underway to document the nature of this sleep disturbance, and explore the pathophysiologic relationship to AFP.

\section{P-152}

Single Photon Emission Computerized Tomography (SPECT) In Patients with Atypical Facial Pain (AFP): A Study of Regional Cerebral Blood Flow (rCBF) Using 99m'Tc-ethyl Cysteinate Dimer (ECD)

\section{StiRling, M. ICHISE, M. Shanduing (Toronto, Ontario)}

Background: Changes in brain metabolism have been observed using Positron Emission Tomography (PET) in AFP, and SPECT in chronic pain patients, suggesting thalamic hyperactivity and focal cortical deficits in the pathophysiology of central origin pain syndromes. Depression is also common in patients with chronic pain, and has been associated with rCBF global hypoperfusion, with frontal predominance. We wish to determine if either objective abnormality is present in patients with AFP using ECD-SPECT imaging.

Methods: Satisfying IHS criteria, 12 consecutive patients diagnosed with AFP (12F; mean age $39.7 \mathrm{yrs}$ ) and screened with the Beck Depression Inventory(BDI), were imaged with ECD-SPECT. The rCBF was compared to that of normal controls.

Results: One of 12 patients displayed mild(17), 5/12 moderate $(23.8 \pm 2.4)$ and $2 / 12(40 \pm 7.1)$ severe depression on the BDI. Eleven had normal rCBF and one(BDI of 45) had a global decrease in $\mathrm{rCBF}$ with ECD-SPECT.

Conclusions: These results do not show significant SPECT abnormalities and despite a high prevalence of depression, the typical SPECT findings in depression were not seen. This could imply either absence of central mechanisms in AFP or lack of sensitivity of ECD-SPECT in this disorder. The clinical utility of ECD-SPECT in AFP remains uncertain. Further investigation, such as thalamic rCBF quantitative measurement, may elucidate the pathophysiology of AFP.

\section{P.153}

\section{Psychological And Pain Parameters in Atypical Facial Pain} (AFP)

\section{StiRling, M. Shandling (Toronto, Ontario)}

Background: AFP is a chronic, poorly localized pain, without neurologic deficits and poorly responsive to treatment. We hypothesize that patients with AFP, as in other chronic pain syndromes, will demonstrate abnormal psychological parameters.

Methods: Prospectively, 12 consecutive patients, presenting to a Craniofacial Pain Unit, diagnosed with AFP (12F; mean age
39.7 yrs), satisfying IHS criteria, completed the Beck Depression Inventory(BDI) and Short-form McGill Pain Questionnaire(SF-MPQ).

Results: AFP onset was post dental procedure or facial trauma in $4 / 12$ and spontaneous in $8 / 12$ patients. Neurologic examinations were normal in these patients. Eleven of 12 patients reported subjective sleep disturbance with frequent arousals and difficulty falling asleep. One of 12 displayed mild(17), 5/12 moderate(23.8 \pm 2.4$)$ and $2 / 12(40 \pm 7.1)$ severe depression on the BDI with sleep disturbance and "a sense of being punished" most commonly identified. The SF-MPQ demonstrated high sensory $(18.1 \pm 5.6)$ and affective $(6.7 \pm 3.3)$ scores, suggesting more intrusive pain than that experienced by historical controls with post-surgical, labor, musculoskeletal or dental pain. High SF-MPQ affective scores paralleled high BDI scores but high SF-MPQ sensory -pain scores did not.

Conclusions: AFP is a chronic, intrusive facial pain and is associated with subjective sleep disturbance and depression. Eight of 12 patients with AFP experienced mild to severe depression, but this did not correlate with the SF-MPQ sensorypain scores. Further investigation is proposed to determine the relationships between sensory-pain perception, affect, subjective sleep disturbance and determinants of quality of life in these patients.

\section{P-154}

\section{Myofascial Pain Syndromes Associated With Definite Neurological Diseases}

B.M. Chaudhuri (Montreal, Quebec), P. M. Barton (Calgary, Alberta)

Background: This poster will discuss four patients with distinct neurological disorders, e.g. Cerebral Palsy, Charcot Marie Tooth Disease, and Multiple Sclerosis. All four patients presented with pain conditions which could not be attributed directly to their primary neurologic disorder or to classical pain generating sensory/neurological structures, i.e. nerve root, peripheral nerve, etc.

Methods: Patients were asked to complete a body map of their pain symptoms. Relevant neurological examinations were performed to define causative and perpetuating factors and to rule out deterioration of their present condition or development of a new neurological disorder. On careful muscle palpation signs of: tenderness, referred pain with palpation, twitch responses, etc., were found to be consistent with well defined myofascial pain syndromes. Treatment was recommended to address the pain and any perpetuating factors.

Results: 11 treated patients reported major improvements in their pain complaints.

Conclusion: Myofascial pain syndromes are an often ignored, yet common occurrence in the normal population. In the neurologic population where weakness, spasticity and contractures are prevalent, the stage is set for the development of muscle dysfunction that can cause and /or perpetuate myofascial pain syndromes. Recognition of these pain conditions facilitates appropriate multidisciplinary management. 


\section{Electroencephalogram (EEG) Driven Stimulation (EDS) to Improve Fibromyalgia Pain Symptoms}

B.M. Chaudhuri (Montreal, Quebec), W. Holden (Edmonton, Alberta), S. Donaldson (Calgary, Alberta)

Background: Fibromyalgia is an ill-defined neurological pain syndrome that affects many in the population. EEG-Driven Stimulation (EDS) is an advanced form of biofeedback which uses flashing light as the feedback signal to alleviate some of the symptoms related to fibromyalgia. Specifically, it improved: pain, weakness, fatigue and insomnia. Fibromyalgia symptoms are observed with evoked EEG slowing consisting of high amplitude, high variability and low frequency brain waves. A computerized system uses spectral analysis to monitor the EEG patterns The purpose of our study was to examine the effects of this therapy on fibromyalgia patients experiencing pain after minor trauma (i.e. whiplash).

Methods: Twenty-three consenting individuals, fully informed of this experimental procedure took part in this study. The sample demographics were mixed. The age range was 17 to 63 years and the mean age was 40 years. Patients had EDS on average of 25 sessions.

Results: Nine patients showed significant improvement, eleven showed some improvement and three showed no change in their symptoms.

Conclusions: EDS showed various degrees of improvement in twenty of the study patients. The exact mechanism of action of this interactive light therapy is unknown but certainly warrants further study with the success of this first Canadian preliminary trial.

\section{P-156}

\section{Migraine with Prolonged Aura in a Dizygotic Twin}

Aвayomi Ogunyemi (St. John's, Newfoundland)

Background: Studies of the heritability of migraine have provided little or no information about "migraine with prolonged aura". This report describes the clinical characteristics of the disorder in a dizygotic twin pair who have had recurrent attacks for 14 years.

Methods: Neurological examination and EEG recording were obtained in the female twin during a habitual attack. Neurological assessment was conducted in her twin brother who suffered from a similar type of migraine.

Results: The female twin had attacks of "migraine with prolonged aura" which were more frequent and more intense than those of her brother. During her attack, the neurological examination showed homonymous hemianopsia and hemianesthesia on the right as well as Broca's aphasia. Simultaneous EEG revealed diffuse high-amplitude delta slow waves, maximum in the left frontal-parietal-temporal region.

Conclusion: Genetic influence likely operates in "migraine with prolonged aura". The possibility exists that the female hormones may adversely affect the clinical manifestations of the disorder.
Migraine-Like Symptoms Triggered By Occipital Lobe Seizures: Response to Sumatriptan

Abayomi Ogunyemi (St. John's, Newfoundland), Dermot ADAMS (Corner Brook, Newfoundland)

Background: The relationship of ictal and post-ictal cephalic pain to migraine remains contentious. We hereby report 2 patients whose post-ictal migraine-like symptoms ameliorated in response to oral Sumatriptan.

Methods: Two patients, a 27 year old woman and 41 year old man were referred for assessment of migraine of 2 years and 4 years duration, respectively. The man described a recent episode of "vacant stare" and the woman had sensitivity to bright colours and light. Neurological examination, visual field testing, CAT scan, MRI scan, and prolonged EEG recordings were accomplished for each patient.

Results: The man had a spontaneous seizure with ictal discharge arising from the right occipital lobe. Bilateral occipital spikes, associated with palinopsia was triggered in the woman by photic stimulation. Both patients developed post-ictal symptoms similar to their migraines. The symptoms subsided following treatment with oral Sumatriptan.

Conclusion: Migraine-like symptoms occurring following occipital lobe seizures may share similar pathophysiological substrates with idiopathic migraine. Serotonergic mechanisms may be implicated.

\section{P-158}

\section{Chronic Daily Headache in Children}

\section{S.S. Seshia, E. Sigurdson (Winnipeg, Manitoba)}

Chronic daily headache is well recognized as an entity in adults but not in children. We describe our experience with 9 children with this condition, seen between 1987 and 1995, and followed for periods of 1 - 84 months (median: 10 months).

At the time of the first visit, their ages ranged from 11 years 17 years (median 13 years); there were 5 males and 4 females. The episode had lasted 1 - 36 months (median 6 months). Severity was often $10 / 10$. Seven had missed a great deal of school. However, there was a striking contrast between headache severity and in ability to attend school on the one hand and facility to do general activities on the other. Either previously or subsequently, 5 had other headache episodes consistent with migraine and 4 with tension type. Clear-cut stressors were identified in three. A family history of migraine was obtained in 4 , of tension type in 1 and non specific headache in 1. Possible response to repetitive DHE, transient in one, occurred in $2 / 3$ children. Almost all interacted with a psychiatrist. The majority have resumed near normal routines despite either continuous or episodic headaches. We suggest that chronic daily headache be recognized as a specific headache disorder by the IHS. 


\section{Clinical Neurological Sciences on the INTERNET}

\section{B.S. JHAWAR, W. Davis (London, Ontario)}

Background: Through the World-Wide-Web, the Internet has become a new media for medical information transfer and communication. Over the past few years, there has been tremendous expansion of clinically relevant material available about the Neurosciences on the Web.

Method: Through joint efforts with the University Computing services, the Department has set up a World-Wide-Web Site representing the interests of Clinical Practioners at the University.

Results: The site gives a brief introduction to the Department and lists its faculty members along with their areas of interest. In addition, the names of residents and clinical nurse specialists are also included. A full description of the various residency programs is provided along with a schedule of weekly rounds and upcoming speakers. Faculty publications and research areas of excellence are briefly outlined. From an educational point, the infrastructure is in place for access to brief tutorials and Grand Rounds cases. The site also acts as a launch site to other relevant locations on the Internet. Another important contribution is the opportunity community physicians have to make quick and accurate patient referral.

Conclusion: The Internet has become an exciting new resource for the medical practitioner. The multi-media format allows both written and graphical representation of unique and highly specialized areas of knowledge which is particularly relevant to clinical neuroscience. It provides an easy means for education of not only other specialists and general physicians, but also for the patient community. This technology has already begun to revolutionize the way in which we learn and teach, the future only holds greater promise.

\section{P-160}

\section{Thomas Willis \& His Circle of Friends}

\section{J. SIDIDIQI (London, Ontario)}

The 'circle of Willis' is an anatomical structure well known to neuroscientists, representing one of numerous discoveries by Thomas Willis that continue to influence contemporary clinical practice. Interestingly, personal and professional collaborations with a diverse group of Oxford colleagues of varying medical and non-medical backgrounds, was critical in the works of Willis. Among the friends of Willis who directly or indirectly contributed to his major achievements can be counted Richard Lower, Christopher Wren, Robert Boyle, Robert Hooke, and Thomas Millington. As a result of his circle of friends, Willis found himself in a milieu of great cross-fertilization of ideas from anatomy, chemistry, philosophy, architecture, and mathematics. We offer details about Willis' inner circle, and document their contributions to his seminal theoretical and experimental studies that linked anatomical fact with clinical practice.

\section{Sir William Osler as an Protagonist of Medical Chirurgical Neurology}

W. FEINDEL (Montreal, Quebec)

Background: The Minutes of the annual meeting in 1879 of the Canadian Medical Association, in London, Ontario stated: "On the morning of September the 1lth, Dr. Osler of Montreal, gave a description of the anatomy of the brain, illustrating his remark by specimens preserved by Giacomini's new process". These lines exemplify Osler's life-long interest in the nervous system and its disorders. The present report documents how he was also a keen protagonist of the emerging field of neurosurgery.

Observations:

1. More than a third of Osler's bibliography concerns neurological disorders.

2. Osler supported the early operations for brain tumor by Rickman Godlee and for epilepsy by Victor Horsley

3. He was mentor to Harvey Cushing, who later became Osler's Boswell.

4. He became hero-figure for Wilder Penfield.

5. He knew William Morris, later Lord Nuffield, who funded Oxford University to establish a Neurosurgical Unit under Sir Hugh Cairns.

6. Osler induced the Rockefellers to support McGill's Medical School which they did in 1920 and again in 1934 when the Montreal Neurological Institute was founded.

Conclusion: Sir William Osler, through his friendship with neurologists, his encouragement of young neurosurgeons, and his contributions to neurological knowledge strongly promoted the development of medical chirurgical neurology as a specialty.

NEUROLOGICAL NURSING - GENERAL

P-162

Landau - Kleffner Syndrome: Strategies from a Nursing and Technical Perspective. (CANN)

L.J. Macmillan, A. Hunjan

P-163

CHAT: Community and Hospital Against Trauma. (CANN)

S. Burns, L. GOULD, H. RENSHAW

P-164

The Effectiveness of an Elementary School Based Education Program on Bicycle Helmet Use in the Community: A City of London Evaluation. (CANN)

B. O'Farrell, R. Alder, T. Stefanits, P. Lane, K. Carty 
P-165

Pyrexia: Implementing Research - based Practice in the Critical Care Setting. (CANN)

J. CROSS, J. FuLCHER

\section{P.167}

Monitoring of Vasospasm in Stroke using Transcranial Doppler Ultrasonography. (CANN)

N. NEWCOMMON

P.168

Sagittal Venous Thrombosis. (CANN)

M. MICHENKO

P-169

Neuroscience Nursing: An International Experience. (CANN)

W. BLACKBURN

\section{P.170}

Florence Nightingale, Yesterday, Today and Tommorrow. (CANN)

W.J. KOOPMAN, D. THRASHER

\section{P-171}

The Stressors Experienced and the Coping Strategies Used by Nurses while Providing Care to Organ Donors and their Families. (CANN)

M. HIBBER'T

\section{P-172}

Discharge Planning: Who, What, Where, When and Why?

C. COCCA, D. THRASHER

\section{P-173}

Paediatric Utilization Protocols: An Effective and Efficient Multidisciplinary Approach to Myelomeningocele Care.

S. LUSCHER, L. PENDERGAST

\section{P-174}

A Spinal Cord Assessment Tool for the Staff Nurse.

P. Doyle-PetTypiece, K. Pugh, G. TOMaszewski
P-175

Coiling as a Non-surgical Intervention for Aneurysms.

C. HARTLEY

\section{SURGICAL CEREBROVASCULAR}

\section{P-176}

Familial Cerebral Aneurysms: Gender Bias for Women

R. Leblanc (Montreal, Quebec)

Background \& Methods: We have evaluated the influence of gender on the formation and rupture of familial cerebral aneurysms by studying 30 patients with ruptured cerebral aneurysms from 14 consecutive families. These patients were compared to the patients with sporadic aneurysms by the Cooperative Study.

Results: Eighty percent of familial aneurysms occurred in women, versus $59 \%$ for sporadic aneurysms $\left(\mathrm{p}<0.05\right.$ chi $^{2}$ test). This over-representation of women occurred below the age of 50 years where $78 \%$ of patients with familial aneurysms were women compared to $45 \%$ for sporadic aneurysms $\left(\mathrm{p}<0.01\right.$, chi $^{2}$ test). Above this age there was no statistical difference in incidence of familial aneurysms in men or women compared to sporadic aneurysms. In women with familial aneurysms, rupture occurred before the age of 50 in $59 \%$, compared to $31 \%$ for sporadic aneurysms ( $p<0.01$, chi ${ }^{2}$ test). In 4 of 5 families $80 \%$, aneurysms ruptured within 10 years of each other in sisters (mean 6 years). Multiple aneurysms were equal in both groups (19\%) but multiple familial aneurysms occurred mainly in women. There was no difference in the site of single cerebral aneurysms in either group but familial aneurysms in females occurred at the same site in 5 of 8 families $(62 \%)$ and in 11 of 17 mother-daughter or sister pairs (65\%), compared to $20 \%$ for two randomly selected sporadic aneurysms $(p<0.01)$.

Conclusions: There is an over-representation of women with ruptured familial aneurysms compared to sporadic aneurysms. Familial aneurysms rupture in females predominantly before the age of 50, and in the same decade and at the same site within families in the majority of cases. These observations support a possible genetic etiology for cerebral aneurysms and a possible hormonal contribution to their rupture.

\section{P-177}

The Efficacy of an Abbreviated Course of Nimodipine Therapy in the Setting of Good-Grade Aneurysmal Subarachnoid Hemorrhage

\section{B. D. ToYota (Vancouver, British Columbia)}

Through prospective clinical trials Nimodipine has been proven to be a valuable adjunct in the management of aneurysmal subarachnoid hemorrhage (SAH). At a dose of $60 \mathrm{mg}$. every four hours for 21 days, patients taking Nimodipine will exhibit a better neurologic 
outcome compared to those not taking the drug. However it has become common clinical practice to provide 'good-grade' aneurysm patients with an abbreviated course of Nimodipine on the basis of cost, convenience and avoidance of side-effects. The efficacy of this practice has not been established. At the University of British Columbia, Division of Neurosurgery, fifty consecutive patients suffering Hunt and Hess grade I-III aneurysmal SAH were treated with an abbreviated course of Nimodipine (range 10 to 15 days) using the standard dosage schedule (60 mg. every four hours). All patients were treated with early aneurysm clipping and post-operative hypervolemic prophylaxis in addition to the Nimodipine. Patients who pursued uncomplicated post-operative courses were discharged within seven to ten days of surgery along with discontinuation of Nimodipine treatment. None of the fifty patients developed delayed neurologic complications of any degree following cessation of therapy and at three months follow-up assessment.

The literature cites vasospasm rates of 13 to $33 \%$ in those patients with Hunt and Hess grade I - III aneurysmal subarachnoid hemorrhages who are not treated with Nimodipine. Our study has shown that 'good-grade' patients, who exhibit no early complications, can safely have their Nimodipine treatment stopped following 14 days of therapy.

\section{P-178}

\section{Retrospective Comparison of Surgical and Guglielmi Detachable Coil Treatment of Saccular Aneurysms}

\section{S.P. LownIE (London, Ontario)}

Background: Detachable coil treatment of cerebral aneurysms has emerged as a potential therapeutic alternative to standard surgical clipping.

Methods: A retrospective review of all patients undergoing surgical or Guglielmi detachable coil (GDC) treatment of saccular intracranial aneurysms by the author from July 1992 to December 1995.

Results: The surgical group consisted of 122 patients (156 aneurysms); the GDC group 22 patients (23 aneurysms). Preoperative grade, aneurysm location and size were similarly distributed between the two groups. Clinical outcomes (all grades) were $80 \%$ excellent/good in the surgical group, $77 \%$ excellent/good in the GDC group. Poor outcome due to the procedure occurred in $6 \%$ of the surgical patients, none of the GDC patients. Aneurysm occlusion was complete in $80 \%$ of surgical aneurysms, neck remnant $12 \%$, significant residual $3 \%$, and unknown $5 \%$. Occlusion was complete in $43 \%$ of GDC aneurysms, neck remnant $35 \%$, significant residual $22 \%$. One of the 24 incompletely clipped aneurysms regrew at 8 months. Of 13 incompletely coiled aneurysms, one rebled and died, one probably rebled and died, and one regrew and was coiled again.

Conclusions: (1) Surgical clipping and GDC treatment of cerebral aneurysms were associated with similar overall clinical outcomes. (2) GDC treatment carried a lower risk of procedurerelated poor clinical outcome. (3) Surgical clipping resulted in a higher chance of complete aneurysm occlusion and a lower risk of aneurysm regrowth or rebleeding and death in the event of incomplete occlusion.

\section{P-179}

Endovascular Treatment of Carotid-Ophthalmic Aneurysms with Guglielmi Detachable Coils (G.D.C.)

D. Roy, J. Raymond, D. Venne, M.W. Bojanowski, G. L'EsPÉRANCE (Montreal, Quebec)

To evaluate the safety and efficacy of endovascular treatment of carotid-ophthalmic aneurysms, we did a prospective study of 25 patients ( 27 aneurysms) treated with G.D.C. coils. Immediate clinical and anatomical results, complications as well as clinical and anatomical follow-ups were registered. There were 22 females and 3 males. Age ranged from 29 to 76 years (mean: 52). Clinical presentation was subarachnoid hemorrhage in 9 patients (ruptured group), mass effect in 1 patient and incidental in 15 patients (non-ruptured group). In the latter group (16 patients with 18 aneuryms), more than $95 \%$ obliteration was obtained in 15 aneuryms (complete in 9) and $70 \%$ in 1 case. These results were stable on control angiograms. No hemorrhage occured in this group. There were 2 failed treatments due to the width of the aneurysmal neck. Four patients did not have their 6 month follow-up angiogram. Clinical follow-up range from 2 to 27 months (mean: 12.3). There was 1 permanent complication with a minor deficit. In the ruptured group more than 98\% obliteration was obtained in 7 cases and $90 \%$ in 1 case. There was 1 failed treatment. Five patients had an excellent outcome (GOS I). Three elderly patients died of their hemorrhage including the operated patient. Follow-up is unavailable in 1 patient. There were no recurrence of hemorrhage with a mean follow-up of 8.4 months. There were no complications related to treatment in this group. Embolization with G.D.C. coils appears to be a safe and efficient alternative to surgery for carotidophthalmic aneurysms.

\section{P-181}

Acute Human Histopathologic Study of an Aneurysm Treated by Guglielmi Detachable Coils - A Case Report

P.J. Porter, S.A. Stiver, R.A. Willinsky, M.C. Wallace (Toronto, Ontario)

Background: The advent of the Guglielmi Detachable Coil (GDC) advanced endovascular therapy as a valuable mode of aneurysm treatment. Despite encouraging early results, a lack of knowledge of the time course and completeness of aneurysm obliteration remains a concern. Histopathologic studies provide an opportunity to explore the effects of coiling on the aneurysm sac, neck, and parent vessel-aneurysm interface. Pathologic studies in animal models have been reported, but there is a paucity of such studies in humans and no reports on human histopathology in the acute phase post-coiling.

Methods: We examined the gross and microscopic pathology of a ruptured $5 \mathrm{~mm}$ anterior communicating artery aneurysm in a patient who died 36 hours following coiling.

Results: Grossly, there was evidence of subarachnoid and intraparenchymal hemorrhage. Thrombus adherent to the aneurysm dome externally was dissected away, revealing loops 
of tightly packed coils covered only by an extremely thin adventitial layer. Opening the parent vessels revealed a shiny, smooth, tan-coloured membrane completely covering the aneurysm orifice. Removal of the coils through the dome followed by histochemical staining of sections demonstrated fibrin and thrombus formation within the aneurysm sac. Across the aneurysm neck was a continuous, fine, reticular membrane composed of fibrin, in continuity with the vessel wall and separating the aneurysm from the parent vessel lumen.

Conclusions: We believe this case demonstrates the early stages of neointima formation. The observed membrane, together with red thrombus in the aneurysm sac, provide histologic evidence for exclusion of the aneurysm from the circulation. In our opinion these findings explain the observed early protection from rebleed provided by coiling. Further studies of the early histologic changes after aneurysm coiling are critical to advance our knowledge of the aneurysm-parent vessel interface, and thereby allow us to predict more accurately the risks of rebleed and embolic complications.

\section{P.182}

\section{Spontaneous Regression of Superior Cerebellar Artery Aneurysms}

\section{R. Leblanc, G. MoHR (Montreal, Quebec)}

Background: Peri-mesencephalic subarachnoid hemorrhage (SAH) is a recently recognized, relatively common cause of $\mathrm{SAH}$ in the absence of an angiographically demonstrable aneurysm. The etiology of peri-mesencephalic SAH is unknown. We report 2 cases that suggest that it may result from the spontaneous regression of a small distal aneurysm of the posterior circulation.

Observations: The first case is that of a 42 year old woman with a Grade I SAH from an angiographically-proven, $.5 \mathrm{~cm}$ aneurysm arising $2 \mathrm{~cm}$ from the origin of the superior cerebellar artery (SCA). The aneurysm was no longer seen on follow-up angiography 10 days and 3 months after the hemorrhage, no specific treatment having been administered in the interval.

The second case is that of a 72 year old man with a grade I $\mathrm{SAH}$ from an angiographically-proven, $.75 \mathrm{~cm}$ aneurysm arising $\mathrm{I} \mathrm{cm}$ from the origin of the SCA. The aneurysm was not seen at surgery 10 days after hemorrhage despite visualization of the SCA from its origin to its first branching point, nor could it be seen the following day at angiography.

Conclusions: Angiographically occult cerebral aneurysms are generally giant in size and thrombosed, presenting not with SAH but with mass effect, which was not the case in our patients. Spontaneous regression may have been prompted by focal vasospasm in our cases. A spontaneously regressed, small, distal SCA aneurysm may be the pathological substrate of angiographically occult peri-mesencephalic SAH. Surgery should be delayed for at least 10 days and angiography repeated at that time in patients with SAH from a distal SCA aneurysm as the lesion may spontaneously regress in the interval.

\section{P-183}

Simultaneous Aneurysmal Subarachnoid Hemorrhage and Basilar Artery Dissection: A Case Report

\author{
H. Bader, T. Zwimpfer, M. Zis, B. D. ToYota (Vancouver, \\ British Columbia)
}

Negative angiography in the setting of a clearly identified subarachnoid hemorrhage remains a clinical and therapeutic dilemma even with the current sophistication of neurodiagnostic technology. There are several important causes for a negative angiogram, and each must each be considered for a given patient. The authors describe the case of a 60 year-old male who presented with a coma-producing subarachnoid hemorrhage. The initial 4-vessel angiogram revealed only a basilar dissection which was presumed to be the source of hemorrhage. There was no evidence of aneurysm, arteriovenous malformation, fistula or vasospasm. On subarachnoid hemorrhage day three a second angiogram was performed with similar findings. A third angiogram was subsequently performed again showing evidence of a basilar dissection as well as a mild degree of vasospasm in both $A_{1}$ segments. The patient suffered a second hemorrhage leaving him moribund. Life-support was then withdrawn. At autopsy a completely thrombosed $8 \mathrm{~mm}$. left carotid artery bifurcation aneurysm was found. The basilar artery dissection was restricted to the sub-intimal layer without subarachnoid extension.

This case represents a unique instance of simultaneous aneurysmal subarachnoid hemorrhage with basilar artery dissection. Mechanisms for this occurrence will be discussed. Furthermore it highlights a dangerous cause of a negative angiogram in the face of a true subarachnoid hemorrhage; that is, a completely thrombosed aneurysm. The authors discuss the causes of negative angiography in the setting of true subarachnoid hemorrhage and propose that magnetic resonance imaging be an requisite part of the investigation for these patients.

\section{P-184}

\section{'Radial' Clipping of Broad-based Aneurysm Necks}

\section{J. SidDiQI, S. LownIE (London, Ontario)}

Background: The conventional surgical treatment of intracranial aneurysms involves the placement of a clip of an appropriate length across the aneurysm neck. In the case of wide necked aneurysms this is frequently awkward; it is occasionally associated with post-operative complications due to clip slippage, or vessel kinking with lumen compromise

Methods: From a series of 35 middle cerebral artery aneurysms operated on over the past 3 years, three have been clipped using a radial technique, with the aneurysm neck being at the epicentre of multiple Sugita clips.

Results: The first patient had undergone clipping of a wide necked aneurysm in the conventional manner, but hemiparesis due to arterial branch kinking was discovered post-operatively. This aneurysm was reclipped using the radial technique, with relief of the branch kinking. Two other patients with large aneurysms have been treated using this method. 
Conclusions: This method of clipping may be safer for certain aneurysms, especially when the integrity of the branching vessels is threatened by conventional clipping. It may be ideal for wide-necked MCA aneurysms because the clips can be positioned vertically, along the axis of the Sylvian fissure, without fears about clip movement post-operatively.

\section{P-185}

\section{Extracranial Internal Carotid Artery Mycotic Aneurysms}

\section{J. SIDDIQI, S. LownIE (London, Ontario)}

Background: Twenty-six verified cases of mycotic aneurysms of the cervical carotid arteries have been reported in the English-language literature. Most of these cases have some known source of infection such as post-operative infection, septicemia, dental abscess, endocarditis or drug addiction. The most common pathogen is staphylococcus, and only four cases attributable to Salmonella have been reported. There are no reported cases of more than one pathogen in the same patient.

Methods and Results: We report the twenty-seventh verified case of mycotic aneurysm of the cervical carotid artery, which presented as an expanding, painful, neck mass associated with high fever and diarrhoea. The lesion was diagnosed with carotid ultrasound and cerebral angiogram, and treated initially with intravenous antibiotics and heparin followed by surgical endaneurysmorrhaphy with excellent clinical outcome

Conclusions: To our knowledge, this is the first case in the literature in which two distinct pathogens were isolated: salmonella gram negative rods in blood and stool; gram positive cocci in the aneurysm tissue itself. It is only the fifth case of salmonella-associated mycotic aneurysm. Our choice of medical and surgical treatments is discussed.

\section{P-186}

Excision of Cerebral Angiomas by the Aid of Cortical Stimulation, Intraoperative Fluorescein Angiography and Cortical Blood Flow Measurement: Two Patients with Follow-up of 28 and 13 Years Respectively

\section{W. FeINDEL (Montreal, Quebec)}

Background: Penfield and Elvidge (1938) reported the early use of cortical stimulation in excision of angiomas located in vital cortical areas. Intraoperative fluorescein angiography (Feindel, Hodge and Yamamoto, 1966) was developed to identify feeding arteries to angiomas, combined with measurement of cortical blood flow by radioactive tracers to document cerebral steal and its reversal after arteriovenous shunt obliteration.

Methods: The technique of intra-carotid fluorescein angiography (ICFA) (Hodge, Yamamoto and Feindel, 1978) was applied to define the epicerebral circulation during craniotomy. Micro regional cortical blood flow was measured by Xenon-133 clearance (Feindel and Yamamoto, 1973).

Observations: Case 1-Male, age 30, with left-sided seizures. Pea-sized angioma located in lower sensory cortex with red draining vein, arteriovenous shunt and cerebral steal shown by ICFA. Excision with retention of blood supply to sensory cortex. Recovery of pre-operative sensory deficit in hand and seizure-free 28 years later.

Case 2 - Male, age 20, with dysphasic seizure from angioma located in the left posterior temporal area. With ICFA, identity and selective clipping of feeding arteries and subsequent excision. Blood supply to speech area identified and preserved. Seizure-free with normal speech 13 years later.

Conclusion: The combined techniques of cortical stimulation, fluorescein angiography and blood flow measurement provide an exact means for obliteration and excision of angiomas with preservation of blood supply to vital cortex.

\section{P-187}

The Classification and Presentation of Intracranial Dural Arteriovenous Fistulas

M.A. Davies, K. TerBrugge, R. Willinsky, M.C. Wallace (Toronto, Ontario)

A number of classification schemes for Intracranial Dural Arteriovenous Fistulas (ICDAVF) based on radiological anatomy have been published, and claim to predict which lesions will present in a benign or aggressive fashion. We have tested the validity of two proposed classification schemes for the first time in a large single institution study.

A series of 102 ICDAVF in 98 patients assessed at a single institution was analysed. All patients were classified using two grading scales: the more descriptive schema of (a) Cognard et al (Cognard*), and (b) that recently proposed by Borden et al (Borden*). Intracranial Hemorrhage (ICH), or Non Hemorrhagic Neurological Deficit (NHND) were considered aggressive presenting clinical features. A total of 16 ICDAVF presented with hemorrhage. Eleven of these hemorrhages (69\%) occured in either Anterior Cranial Fossa or Tentorial lesions but simply reflected the number of higher grade lesions in these locations. When analysed according to the Borden* classification, $0 \%$ grade I, $11 \%$ grade II, and $48 \%$ grade III ICDAVF presented with hemorrhage $(p<0.0001)$. After exclusion of visual or cranial nerve deficits clearly related to cavernous sinus ICDAVF, NHNDs were a feature of presentation in $2 \%$ grade I, $28 \%$ grade II, and $31 \%$ grade III patients $(p<0.0001)$. When combined, an aggressive clinical presentation strongly correlated with Borden* grade: $2 \%$ grade I, $39 \%$ grade II, and $79 \%$ grade III patients $(\mathrm{p}<0.0001)$. A similar correlation with aggressive presentation was also seen with the Cognard" classification: $0 \%$ grade I, $7 \%$ grade IIA, $37 \%$ grade IIB, $40 \%$ grade IIA $+\mathrm{B}, 69 \%$ grade III, $83 \%$ grade IV, $100 \%$ grade V. $(\mathrm{p}<$ $0.0001)$.

No location is immune from harbouring lesions capable of an aggressive presentation. Location itself only raises the index of suspicion for dangerous venous anatomy in some ICDAVF. The configuration of venous anatomy as reflected by both the Cognard* and Borden* classifications strongly predict ICDAVF that will present with ICH or NHND. 


\section{P-188}

Intracranial Dural Arteriovenous Fistulae with Pial Venous Drainage: Combined Endovascular Neurosurgical Therapy

D.M. Pelez, S.P. Lownie, D. Rosso, A.J. Fox, (London, Ontario)

Background: Intracranial dural arteriovenous fistulae (AVF's) with pial venous drainage are potentially dangerous lesions which are difficult to treat. We wish to emphasize the utility of a combined endovascular -neurosurgical approach to their treatment.

Methods: The clinical and radiological records of all patients who presented to our institution with intracranial dural AVF's over the last 5 years were reviewed. In those cases demonstrating pial venous drainage, details of presentation, imaging features, endovascular and surgical therapy and outcome were analyzed.

Results: We identified 12 patients with these lesions, 7 of whom presented with subarachnoid hemorrhage. Six patients were treated with embolization alone. Angiographic cure was achieved in 4 . There was one complication in this group, a subarachnoid hemorrhage following glue injection. Six patients were treated with embolization followed by surgical ligation of the pial venous drainage. Angiographic cure was achieved in all 6 . There was one complication in this group, a facial nerve palsy following glue injection.

Conclusion: Endovascular therapy of intracranial dural AVF's may be curative but is often complex and carries definite risks. Neurosurgical ligation of pial draining veins, with preoperative embolization when safe, may be a relatively more controlled method to achieve complete cure.

\section{P-189}

Stereotactic Radiosurgery versus Observation for Symptomatic, Surgically Inaccessible Cavernous Malformations of the Brain - Proposal for a Double Blind, Randomized, Sham-Controlled Trial

\section{P.J. Porter, M.C. Wallace (Toronto, Ontario)}

Background: Cavernous malformations (CMs) of the brain typically become symptomatic at a young age, and have a high annual risk of causing neurologic deficit or death. If the location does not permit operative removal, there is currently no proven effective treatment. Preliminary studies using stereotactic radiosurgery (SR) for inoperable CMs which had previously hemorrhaged have found a marked reduction in rebleeding with acceptable adverse effects from treatment. However, no controlled trial has been performed.

Methods: Eligible patients with symptomatic, surgically inaccessible CMs will be recruited from participating neurovascular clinics across North America and randomized to either SR or a sham procedure. Only one clinician, not involved in subsequent care of the patient, will be aware of treatment status. Patients will be followed for a minimum of 5 years by blinded clinical assessments, self-administered quality of life (QOL) questionnaires, and serial MRIs. The required sample size is 186 (93 patients per group) for statistical analysis of primary and secondary outcomes with $80 \%$ power and $5 \%$ Type I error rate. With 10 multidisciplinary groups participating, the estimated accrual period is three years, making the total trial duration 8 years.

Results: The primary outcome is permanent neurologic morbidity (both hemorrhagic and non-hemorrhagic) and death. The secondary outcome is temporary morbidity. Exploratory analyses will assess differences in QOL and radiologic changes. Outcomes will be verified by an independent, blinded adjudication committee. A Data and Safety Monitoring Committee will be operational to ensure safe and efficient trial progress.

Conclusions: A randomized controlled trial is required to definitively answer the question of whether SR improves the prognosis of patients with symptomatic, inoperable CMs. The trial proposed herein is feasible and ethically justifiable, and will provide a solution to this problem which commonly plagues vascular malformation specialists.

\section{P-190}

Hereditary Neurocutaneous Vascular Hamartomas: Delineation of a Syndrome with Autosomal Dominant Inheritance

\section{R. LeBLANC, D. MELANSON, R. WiLkinson (Montreal, Quebec)}

Background: We report the co-existence of vascular naevi (haemangiomas and arteriovenous malformation [AVM] of the skin) with AVMs and venous malformations of the brain in male siblings from 2 related but nonconsanguineous families.

Methods: The proband, his siblings, parents, aunts, uncles, and cousins were examined and underwent magnetic resonance imaging and magnetic resonance angiography, and, when appropriate, cerebral angiography.

Results: A father had vascular naevi and a mother an azygous anterior cerebral artery. No other cutaneous or cerebral vascular malformations were present in the parents. The two families had 3 children, 2 boys and 1 girl each, aged 9-18 years. All the children had vascular naevi and all of the boys had coexisting cerebro-vascular malformations: AVMs in 3, a venous malformation in another. One body had 3 cerebral AVMs. Two boys had a cerebral haemorrhage and one also had focal motor seizures. The skin lesions were not those of the Sturge-Weber-Dimitri, Rendu-Osler-Weber or Wynburn-Mason syndromes.

Discussion: The association of cutaneous and cerebral vascular malformations was seen only in males in our families but females with both conditions have also been reported. Our cases suggest that the association of cerebral and cutaneous vascular hamartomas constitute a distinct hereditary clinico-pathological entity with autosomal dominant inheritance and variable penetrance. The clinical manifestations of this syndrome are visible, painful vascular naevi, epilepsy, cerebral haemorrhage and focal neurological deficits.

The male preponderance of patients with the full expression of the syndrome suggests a possible hormonal influence on the expression of the gene. 
Superficial Cerebral Hemosiderosis and Cavernous Angiomas: A Case Study and Review of the Literature

\section{J. SiddiQI, M. Abdulhak, G. Ferguson (London, Ontario)}

Background: Superficial cerebral hemosiderosis ( $\mathrm{SCH}$ ) is a rare disease of the central nervous stem characterized by deposition of hemosiderin in the leptomeninges, spinal cord and cranial nerves, commonly presenting with sensorineural deafness, cerebellar ataxia and pyramidal signs. This condition may be due to idiopathic subarachnoid hemorrhage (47\%) or some other identifiable source of bleeding (53\%) accompanying dural or vascular pathology.

Methods and Results: We present the case of a 52-year old male with $\mathrm{SCH}$ presenting with sensorineural hearing loss, dementia, ataxia and pyramidal signs secondary to recurrent subclinical subarachnoid hemorrhage from multiple congenital cavernous hemangiomas diagnosed by MRI and confirmed at autopsy.

Conclusions: This case represents the first case of its kind in the literature of SCH secondary to recurrent, cavernous hemangioma bleeds. The clinical picture, natural history, diagnosis and therapy are discussed.

\section{P.192}

The Presentation and Surgical Management of Intracerebral Haemorrhage Secondary to Cerebral Amyloid (Congophilic) Angiopathy

P.A. Pahapllil, D.A. Ramsay and R.F. Del Maestro (London, Ontario)

Background: Cerebral Amyloid Angiopathy (CAA) is a wellrecognized cause of spontaneous lobar haemorrhage in the elderly. Because of the aging population, the incidence of intracerebral haemorrhage due to CAA will increase. The treatment of patients with CAA-related intracerebral haemorrhage is controversial. Some reports recommend non-surgical management because of the perceived difficulty in controlling intra-operative haemorrhage and the possiblity of recurrent post-operative haemorrhage.

Methods: We reviewed our surgical pathology files to identify cases of histologically confirmed CAA in which the patients underwent full surgical evacuation of their clots. These were compared with 60 similar cases in the literature. We also reviewed the cases of surgical biopsy without evacuation in patients with CAA.

Results: Two major observations were made. First, over $80 \%$ of patients presented with a single, isolated haemorrhage. Second, the morbidity and mortality of surgical intervention in this group of patients was no worse than for intracerebral or lobar haemorrhage due to other causes.

Conclusions: These findings indicate that the surgical management of cases of suspected CAA-induced lobar haemorrhage should be no different than that of haemorrhage due to other causes.

\section{Comparison of Carotid Surgery in Three Toronto Hospitals}

\section{SmURawska, R. Maggisano, J. NorRIS (Toronto, Ontario)}

Data from recent trials of carotid surgery have emphasised the importance of minimising complications of carotid angiography and endarterectomy (CEA) to maintain a positive riskbenefit ratio. We compared the CEA results in three Toronto teaching hospitals between May 92 and June 95 .

There were 228 consecutive patients undergoing CEA (hospital $\mathrm{A}=50$ patients, hospital $\mathrm{B}=50$, hospital $\mathrm{C}=128$ ). $9.6 \%$ were asymptomatic. $69 \%$ men, mean age $64 \pm 8,31 \%$ women, mean age $70 \pm 9$. Total stroke and death rate was $5.7 \%$, minor complication $11 \%$ (including neck hematoma, cardiac failure, pneumonia etc). Mean length of hospital stay was $8 \pm 6$ days (without complications $7 \pm 5$, with complications $12 \pm 8$ ). There were no significant difference in the data between the three hospitals.

Complication rate was considerably higher than published data, possibly reflecting the bias of clinical trials compared to standard hospital practice.

\section{P-194}

Analysis of Airway Volume Following Carotid Endarterectomy (CEA) Using CT Scans

E.T. Kiriakopoulos, F.J. Carmichael, G.P. McGuire, S. Crofts, M.C. Wallace, W. Montanera (Toronto, Ontario)

Purpose: To prospectively study airway dimensions in CEA patients pre and post operatively.

Methods: The study included 19 patients. Axial CT slices were performed through the neck and extended from the base of the tongue to immediately below the cricoid cartilage. AP and transverse diameters of the airway were measured at 3 points (hyoid bone, arytenoid, cricoid). Edema was categorized as unilateral or bilateral. $\mathrm{CT}$ data were transferred to a workstation for $3 \mathrm{D}$ reconstruction and airway volume assessment.

Results: CT scan demonstrated swelling in all patients. Five patients had clinical evidence of airway obstruction and the presence of a hematoma on CT and were intubated postoperatively. 3D reconstruction of the neck from CT scans showed a reduction in the airway volume in all patients. This reduction was greater in the intubated $(62 \pm 9 \%)$ compared to the non-intubated $(32 \pm 7 \%)$ patients $(\mathrm{p}<0.01)$. AP and transverse diameters of the airway were reduced. Retropharyngeal edema was increased. This change was greater for the upper airway at the level of the hyoid and was significantly greater in the intubated patients.

Conclusions: There is significant soft tissue edema of the neck following CEA that reduces airway volume and can result in airway obstruction. 


\section{P-195}

Carotid Endarterectomy and Thrombectomy in a 29 Year Old Male.

\section{Mehta, R.A. LanGille (Halifax, Nova Scotia)}

Background: Carotid Endarterectomy and Thrombectomy has been described as a surgical treatment for carotid dissection. Current management for dissection of the extracranial carotid artery that has a mural based thrombus without occlusion is varied.

Method: We reviewed an interesting case of a healthy 29 year old male who presented initially with left sided frontal headaches and subsequent left hemisphere dysfunction.

Results: The patient underwent craniotomy for a lesion that on magnetic resonance imaging was suspicious for a left parietal lobe neoplasm. At the time of craniotomy infarcted tissue was encountered. Subsequent investigations revealed a mural based thrombus in the cervical internal carotid artery with a degree of stenosis. Carotid thrombectomy and endarterectomy was done 1 day following the craniotomy. There was a remote history of trivial blunt trauma to the neck.

Conclusions: An index of suspicion for carotid disease should be taken with young patients with neurological signs of hemisphere dysfunction. Management of carotid thrombus is controversial and may include surgical treatment.

\section{P-196}

Perioperative Hemodynamic Instability After Carotid Endarterectomy

\section{J.H. WONG, J.M. FInDLAY (Edmonton, Alberta)}

Objective: To examine perioperative hypertension, hypotension, and bradycardia after carotid endarterectomy with respect to incidence, predictive variables, and associations with postoperative neurologic or cardiac complications, or death.

Design: Retrospective cohort study utilizing hospital chart review of preoperative factors including age, history of cardiac disease and hypertension, as well as hemodynamic data from postoperative days zero and one.

Patients: Eighty-five consecutive patients who were operated over an 18 month period, all monitored in an intensive care unit.

Outcome measures: Postoperative transient ischemic attack, stroke, angina, myocardial infarction, dysrhythmia, congestive heart failure, or death occurring in hospital.

Results: Incidences of postoperative hypertension (systolic BP $\geq 180$ torr), hypotension (systolic BP $<90$ torr), and bradycardia (pulse $<60$ beats per minute) were $46 \%, 7 \%$, and $51 \%$, respectively. Parenteral pressors, antihypertensive medications, or atropine were required in $27 \%$ of patients. Only a history of hypertension reliably predicted postoperative hypertension (relative risk $2.34 ; 95 \%$ confidence intervals 1.23 to 4.44 ). All three observed cerebral ischemic events occurred in patients with bradycardia. No other significant associations between postoperative hemodynamic instability and neurologic or cardiac complications or death were detected.

Conclusions: Hemodynamic instability is a common but usually unpredictable phenomenon after carotid endarterectomy that often requires acute pharmocologic intervention.

\section{P.197}

Neuroprotective Effects of Hypothermia in Generalized Ischemic Encephalopathy

\section{G.B. YounG, D.A. Ramsay (London, Ontario)}

Background: Generalized myoclonic seizures in comatose patients after generalized ischemic encephalopathy have an associated mortality of $97 \%$. Post mortem examination has revealed widespread neuronal injury.

Methods: We present a case series of 3 consecutive patients with aortic rupture who were comatose and had myoclonic seizures after 3.5 to 15 minutes of severe cerebral oligemia. All were promptly treated with intra- operative systemic hypothermia from 16 to 28 degrees C. Patients had clinical and electroencephalographic studies.

Results: An autopsy on the first patient who died 3 days later revealed minimal neuronal damage. The other patients have made nearly complete recoveries.

Conclusions: Systemic hypothermia has potent neuroprotective effects when instituted promptly after the event. Patients so treated should have vigorous treatment of seizures and should be managed expectantly. Previously published prognostic criteria for cases of post-ischemic myoclonic seizures and coma may not be reliable for patients promptly treated with systemic hypothermia.

\section{P-198}

The Development of a Stroke Survivor's Guide. (CANN)

D. BEST

P-199

Bridging A Gap: Stroke Rehabilitation at Parkwood Hospital. (CANN)

S. JANKOWSKI 